UNIVERSIDADE DE SÃO PAULO

ESCOLA DE ENGENHARIA DE SÃO CARLOS

INSTITUTO DE FÍSICA DE SÃO CARLOS

INSTITUTO DE QUÍMICA DE SÃo CARLOS

ÁREA INTERUNIDADES CIÊNCIA E ENGENHARIA DE MATERIAIS

DETERMINAÇÃO DA ESTRUTURA

CRISTALINA E MOLECULAR DE UMA

FLAVONA

Jaime de Souza Júnior

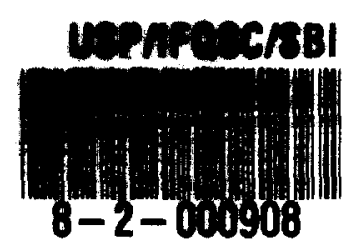

Dissertação apresentada a área interunidades

Ciência e Engenharia de Materiais, IFSC, EESC IQSC,

da Universidade de São Paulo para a obtenção do título

de Mestre em Ciência e Engenharia de Materiais.

OEVICO DE Biblioteca E INFORMACAO

IOSCIUSPT TQOQ

ORIENTADOR: Prof. Dr. Regina Helena de Almeida Santos

SÃO CARLOS

1996 
Área Interunidades

Ciência e Engenharia de Materiais

UNIVERSIDADE DE SÃO PAULO

Escola de Engenharia de São Carlos

Instituto de Física de São Carlos

Instituto de Quimica de São Carlos
CAIXA POSTAL - 369

CEP $13560-970$ - São Carlos/SP - Brasil

Tel/Fax: (016) 274-9285

MEMBROS DA COMISSÃO JULGADORA DA DISSERTAÇÃO DE MESTRADO DE JAIME DE SOUZA JÚNIOR APRESENTADA A ÁREA INTERUNIDADES EM CIÊNCIA E ENGENHARIA DE MATERIAIS, DA EESC-IFSC-IQSC. UNIVERSIDADE DE SÃO PAULO, EM 24/05/1996.

COMISSÃO JULGADORA:

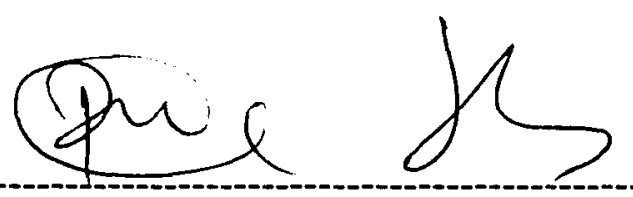

Profa. Dra. Regina Helena de Almeida Santos (DQF.M-IQSC/USP)

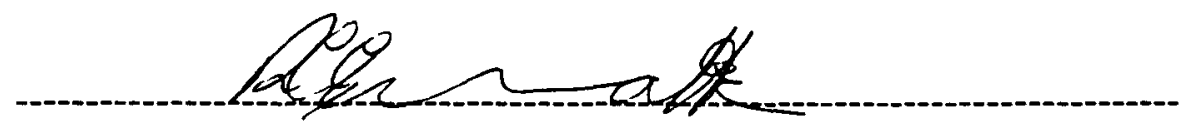

Prof. Dr. Richard Charles Garratt (FFI-IFSC/USP)

Sauls.

Prof. Dr. Nilso Barelli (IQ-UNESP-Araraquara)

CIWINHORD SCMDEFESAS DEFJAIME.DOC 
CAPES, CNPq, FAPESP, FINEP 
Aos meus pais, Jayme e Stella A Deus que me acompanha nesta minha existência 


\section{AGRADECIMENTOS}

Quero expressar meus sinceros agradecimentos a todos aqueles que colaboraram para a execução deste trabalho de uma forma direta ou indireta, e em especial:

A Profa. Dra. Regina Helena de Almeida Santos pela dedicação com que orientou este trabalho, pelos seus ensinamentos, incentivo, apoio e amizade demostrados;

A Profa. Dra. Maria Teresa do Prado Gambardella pela solicitude e amizade;

A Prof. Dra. Regina H. Porto Francisco pela colaboração e amizade;

Ao Prof. Dr. Lourivaldo Santos da Universidade Federal do Pará, pelo fornecimento da amostra;

Aos demais Professores dos grupos de Cristalografia e Quântica pelo incentivo e amizade;

Aos meus colegas de sala Cristina Cunha Carvalho, Patrícia Carolina Moreno e Odonírio Abrahão Júnior, pela amizade, colaboração e companheirismo;

A Vânia Cardoso, Joel Marcondes pela amizade e solicitude;

A Ângela Marcia Derigi Silva pela colaboração, amizade e tempo dispensado;

A Lio Vieira pela sugestões e discussões;

A Evandro Lopes Salgado pela força e amizade;

Ao Prof. Evair pelas sugestõesa e amizade;

A Taís Gauzer pelas sugestões e críticas;

A Dona. Aurora pela amizade;

Aos meus pais, minha eterna gratidão pelo amor, força , paciência, compreensão e incentivo que sempre recebi. 


\section{ÍNDICE}

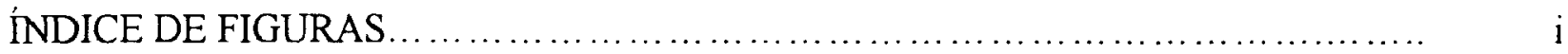

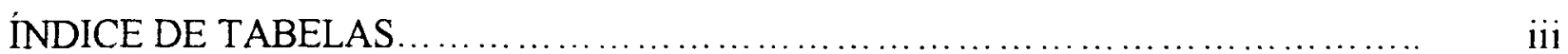

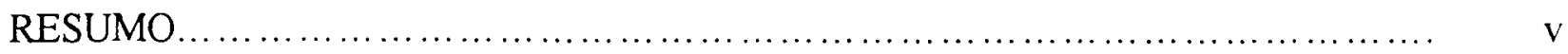

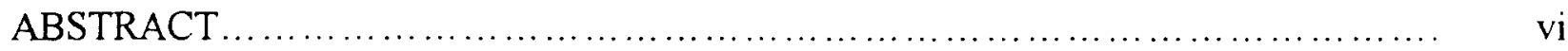

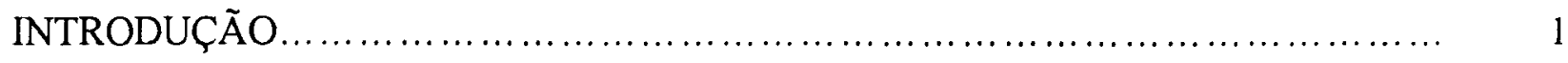

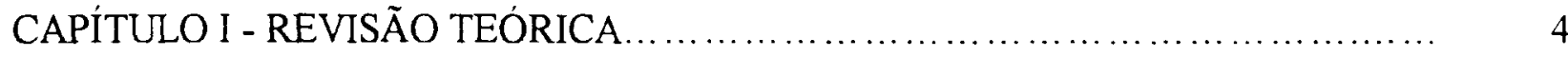

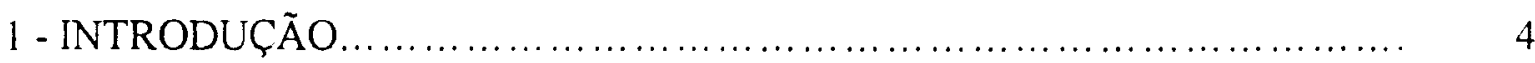

1.1 - ORIGEM DA PRODUÇÃO DE RAIOS-X........................... 4

1.2 - INTERAÇÃO DOS RAIOS-X COM A MATÉRIA ....................... 6

1.2.1- ESPALHAMENTO POR UM ELÉTRON ........................ 6

1.2.2- ESPALHAMENTO POR UM ÁTOMO .......................... 8

1.2.3 - ESPALHAMENTO POR UMA CELA UNITÁRIA ............... 10

1.3 - A REDE RECÍPROCA ........................................ 12

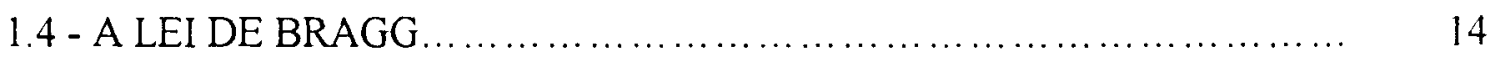

1.5 - AS EQUAÇÕES DE LAUE..................................... 15

1.5.1 - EQUIVALÊNCIA DAS EQUAÇÕES DE LAUE E A LEI DE

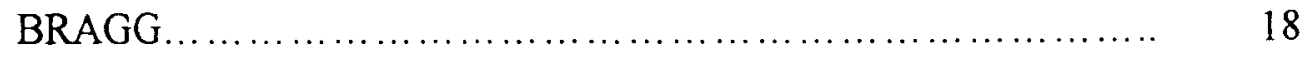

1.6- ESFERA DE EWALD ......................................... 20

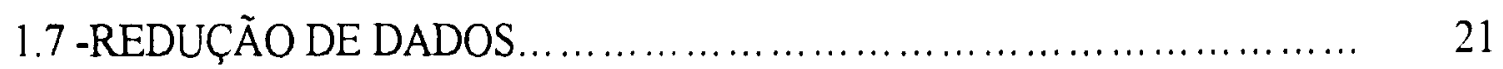

1.7.1 - FATORES QUE AFETAM AS INTENSIDADES ................ 23

1.7.1.1 - FATOR DE POLARIZAÇÃO .............................. 23

1.7.1.2 - FATOR DE LORENTZ ............................... 23 
1.7.1.3 - FATOR DE ABSORÇÃO

1.8 - FATOR DE TEMPERATURA E SUA RELAÇÃO COM A INTENSIDADE

1.9 - DENSIDADE ELETRÔNICA E FATOR DE ESTRUTURA.

1.9.1 -SÉRIES DE FOURIER

1.10 - O PROBLEMA DA FASE.

1.11 - MÉTODOS DIRETOS

1.11.1 - DESIGUALDADES DE HARKER e KASPER

1.11.2 - FATOR DE ESTRUTURA UNITÁRIO

1.11.3 - FATOR DE ESTRUTURA NORMALIZADO

1.11.4 - DETERMINANTE DE KARLE- HAUPTMAN.

1.11 .5 - INVARIANTES ESTRUTURAIS

1.11.6 - SEMI-INVARIANTES ESTRUTURAIS E DEFINIÇÃO DA ORIGEM

1.11.7 - RELAÇÕES DE PROBABILIDADE

1.11.7.1 - MÉTODOS CENTROSSIMÉTRICOS.

1.11.7.2 - MÉTODOS NÃO CENTROSSIMÉTRICOS.

1.11.8 - ADIÇÃO SIMBÓLICA.

1.11.9 - AS BASES DO MÉTODO DE MULTISSOLUÇÃO

1.12 - REFINAMENTO DE UMA ESTRUTURA

1.12.1 - REFINAMENTO PELA SÍNTESE FOURIER DIFERENÇA

1.12.1.1 - SITUAÇÕES LIMITANTES DA FOURIER DIFERENÇA .....

1.12.2 - REFINAMENTO POR MINIMOS QUADRADOS. 
1.12.3.1 CONTRIBUIÇÃO DE NON POISSON

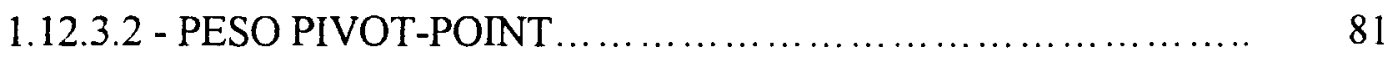

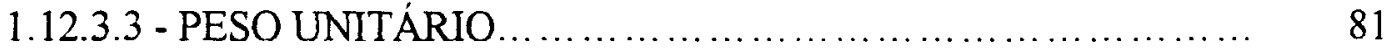

1.12.3.4 - PESO UNITÁRIO MODIFICADO ....................... 81

1.12.3.5 - PESO POLINOMIAL CRUICKSHANK .................... 82

1.12.3.6 - PESO POLINOMIAL MODIFICADO DE CRUICKSHANK... 82

1.12.3.7- MÉTODO DE PESAGEM KILLEAN E LAWRENCE ......... 82

1.12.4 - CONTROLE DO REFINAMENTO E ÍNDICES DE DISCORDÂNCIA

CAPÍTULO II - DETERMINAÇÃO DA ESTRUTURA CRISTALINA E MOLECU-

LAR DO 5,4'- DIHIDROXI - 3',5'- DIMETOXI - 6,7 - (2”, 2’, DI-

METILPIRANO) FLAVONA ................................. 84

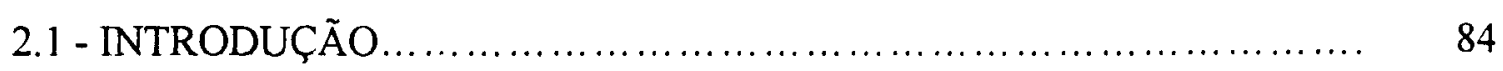

2.2 COLETA DE DADOS ............................................ 85

2.3 - SOLUÇÃO E REFINAMENTO DA ESTRUTURA .................... 87

2.4 - RESULTADOS E CONCLUSÕES............................... 89

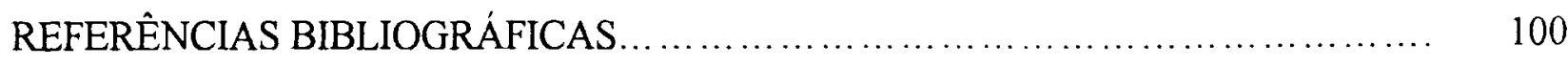

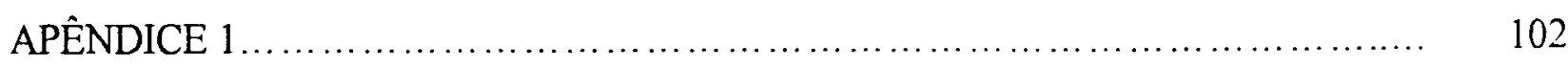




\section{ÍYDICE DE FIGURAS}

Figura 1- Representação estrutural de uma molécula de Flavona, com a numeração típica usada nos flavanóides.

Figura 2- Representação estrutural de alguns compostos da classe dos flavonoides. (a) flavanona; (b) isoflavona; (c) flavonol.

Figura 3- Campos elétrico e magnético associados com o movimento da onda de raios- $X$ na direção do eixo $\mathrm{x}$

Figura 4- Colisão elástica entre fóton e elétron (Demonstração do Efeito Compton)......

Figura 5- Curva do fator de espalhamento para o átomo de carbono..............

Figura 6 - Representação tridimensional de uma cela unitária genérica...

Figura 7 - Adição de ondas: a) tratamento vetorial; b) tratamento analítico; c) ondas senoidais

Figura 8 - Espaço Reciproco. a) representação dos eixos. b) relação entre o vetor $\mathrm{H}$ e o plano hkl

Figura 9 - Construção apresentando as condições de difração $\quad$..................... 14

Figura 10- Diferença de percurso entre os raios incidentes e difratados........... 16

Figura 11- Difração dos raios-X pelos planos cristalinos......................... 19

Figura 12 - A Esfera de Ewald ou Esfera de Reflexão.

Figura 13 - Relação entre os raios- $X$ incidentes e os raios- $X$ refletidos em um elemento de volume $\mathrm{dx}$.

Figura 14 - Gráfico de Wilson para a determinação dos fatores de escala e temperatura isotrópico médio

Figura 15. Densidade eletrônica unidimensional simétrica.

Figura 16 - Ilustração do diagrama de Argand.

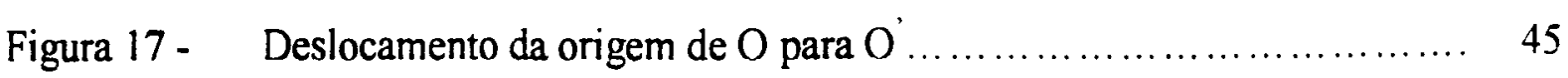

Figura 18- Cela unitária no espaço reciproco................................. 48

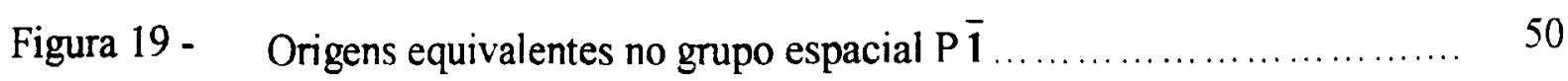

Figura 20- Distribuição de probabilidades de $\phi_{3}$ para três valores de K............ 57

Figura 21 - Diagrama do processo de convergência.......................... 62 
Figura 22 - Efeito dos erros posicionais visualizado na Fourier diferença. (a) na direção de $\mathrm{x}$; (b) no plano xy.

Figura 23 - Situações limites da sintese $\Delta F$. (a) $\left|F_{o}\right| \approx\left|F_{c}\right| ;$ (b) $\left|F_{o}\right|>\left|F_{d}\right| ;$ (c) $\left|F_{0}\right|>\left|F_{c}\right|$

Figura 24 - (a) vetores para o caso $\left|F_{c}\right|>\left|F_{o}\right|$. (b) vetores para o caso. $\left|F_{c}\right|>$ $\left|F_{o}\right| \cdot \operatorname{com} \alpha_{o}=\alpha_{c}$. (c) comparação entre os vetores $F_{0}-F_{c}$ e $\Delta F e^{-i u c} \ldots \quad 72$

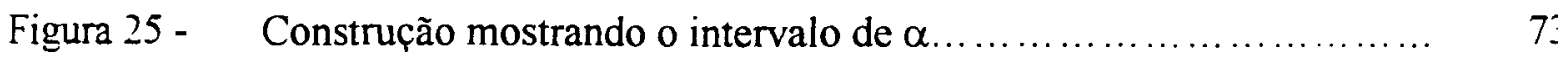

Figura 26 - Seção em linha da síntese $\rho_{\mathrm{o}}-\rho_{\mathrm{c}}$ : (a)- parâmetros térmicos superestimados; (b)-parâmetros térmicos sub-estimados

Figura 27 - Seção da densidade eletrônica por um átomo. (a) densidade eletrònica ideal, usando $F_{0}$; (b) densidade eletronica usando vibração isotrópica $\left(\mathrm{F}_{\mathrm{c}}\right)$; (c) densidade eletrônica $\Delta \mathrm{F}$, mostrando a aparència da diferença que ocorre quando um átomo vibrando anisotrópicamente é considerado isotróicpo. As linhas contínuas representam regiões positivas e as tracejadas regiões de densidade eletrônica negativa.......

Figura 28 - Grafico do limiar para o sistema de peso unitário modificado

Figura 29 - Representação Ortep da molécula de 5,4' - dihidroxi - 3',5'- dimetoxi 6,7 - (2", 2", dimetilpirano) flavona.

Figura 30 - Representação Ortep da Molécula de 5,4'- Dihidroxi - 3',5' - dimetoxi 6,7 - (2", 2", dimetilpirano) flavona com os elipsoides de vibração térmica

Figura 31 - Representação Ortep da molécula de 5,4'-dihidroxi-3',5'-dimetoxi-6,7(2",2"-dimetilpirano) flavona. As linhas tracejadas indicam as ligações de hidrogênio intramoleculares.

Figura 32 - Representação ORTEP das moléculas com orientação relativa à cela unitária, formando cadeia ao longo da direção [101]. As ligações de hidrogênio intra e intermoleculares estão representadas por linhas tracejadas 


\section{ÍNDICE DE TABELAS}

TABELA 1 - VALORES TEÓRICOS RELACIONADOS AOS $|E|$

TABELA 2 - VALORES DE K e E PARA DIFERENTES NÚMEROS DE ÁTOMOS.

TABELA 3 - RESUMO DOS PRINCIPAIS DADOS CRISTALOGRÁFICOS

TABELA 4 - COORDENADAS ATÔMICAS FRACIONÁRIAS E FATORES DE VIBRAÇÃO TÉRMICA ISOTRÓPICOS EQUIVALENTES $\left(\AA^{2}\right)$ COM OS RESPECTIVOS DESVIOS PADRÃO ENTRE PARÊNTESES EXCLUIDOS OS HIDROGÊNIOS PARA O 5,4'DIHIDROXI-3',5'-DIMETOXI-6,7-(2",2"'-DIMETILPIRANO) FLAVONA.

TABELA 5 - COORDENADAS ATÔMICAS FRACIONÁRIAS DOS ÁTOMOS DE HIDROGÊNIO PARA O 5,4'-DIHIDROXI-3',5'-DIMETOXI-6,7-(2", 2"'-DIMETILPIRANO) FLAVONA

TABELA 6 - PARÂMETROS DE VIBRAÇÃO ANISOTRÓPICA PARA O 5,4'-DIHIDROXI-3',5'-DIMETOXI-6,7-(2"',2"'-DIMETILPIRANO) FLAVONA.

TABELA 7 - DISTÂNCIAS INTRAMOLECULARES $(\AA)$ COM OS RESPECTIVOS DESVIOS PADRÃO ENTRE PARÊNTESES PARA O 5,4'-DIHIDROXI-3',5'-DIMETOXI-6,7-(2', 2'”-

DIMETILPIRANO) FLAVONA.

TABELA 8 - ÂNGULOS INTRAMOLECULARES $\left({ }^{\circ}\right)$ COM OS DESVIOS PADRÃO ENTRE PARÊNTESES PARA PARA O 5,4'-DIHIDROXI-3',5'-DIMETOXI-6,7-(2', 2'"-DIMETILPIRANO) FLAVONA. 
TABELA 9 - EQUAÇÕES DOS PLANOS DE MÍNIMOS QUADRADOS E ÂNGULOS DIEDROS PARA O 5,4'-DIHIDROXI-3',5'-DIMETOXI-6.7-(2",2"'-DIMETILPIRANO) FLAVONA.

TABELA 10 - DISTÂNCIAS DE LIGAÇÃO DE HIDROGÊNIO ( INTERMOLECULARES, COM OS RESPECTIVOS DESVIOS PADRÃO ENTRE PARÊNTESES PARA O 5,4'-DIHIDROXI-3' ,5'-DIMETOXI-6,7-(2"',2'"-DIMETILPIRANO) FLAVONA......

Tabela 11 - $\quad$ ÂNGULOS DE LIGAÇÃO DE HIDROGÊNIO $\left({ }^{\circ}\right)$ INTRA E INTERMOLECULARES, COM OS RESPECTIVOS DESVIOS PADRÃO ENTRE PARÊNTESES PARA O 5,4'-DIHIDROXI3',5'-DIMETOXI-6,7-(2', 2''-DIMETILPIRANO) FLAVONA ...

TABELA A1 - FATORES DE ESTRUTURA OBSERVADOS E CALCULADOS PARA O 5,4 -DIHIDROXI-3',5'-DIMETOXI- 6,7 -(2', 2'"-DIMETILPIRANO)FLAVONA. 


\section{RESUMO}

Neste trabalho apresenta-se inicialmente algumas considerações sobre a difração de raios- $X$, suas principais leis, fatores de correção dos dados experimentais, uma revisão sobre os principais Métodos Diretos de resolução da estrutura, e considerações sobre o refinamento da estrutura obtida.

A seguir descreve-se a determinação da estrutura cristalina do produto natural 5,4-Dihidroxi-3',5'-dimetoxi- 6,7 -(2",2"'-dimetilpirano) flavona de fórmula molecular $\mathrm{C}_{22} \mathrm{H}_{20} \mathrm{O}_{7}$, isolado de plantas da espécie Neoraputia paraensis, que cristaliza-se no sistema monoclínico, grupo espacial $\mathrm{C} 2 / \mathrm{c}$, com os seguintes parâmetros de cela unitária: $\mathrm{a}=13,651(1)$, $\mathrm{b}=23,428(2), \mathrm{c}=13,725(1) \AA ; \beta=119,528(4)^{\circ}, V=3819,6(5) \AA^{3}, D_{c}=1,366 \mathrm{~g} \mathrm{~cm}^{-3}$ e $Z=8$ moléculas por cela unitária.

A estrutura foi resolvida através da aplicação de Métodos Diretos. Os índices de discordância finais são: $\mathrm{R}=0,0509, \mathrm{R}_{\mathrm{w}}=0,0530$ para 1743 reflexões com $\mathrm{I} \geq 3 \sigma(\mathrm{I})$.e $\mathrm{R}_{\mathrm{all}}=$ 0,157 .

A estrutura foi refinada fazendo uso dos cálculos de Fourier Diferença e pelo métodos de mínimos quadrados usando matriz completa.

A molécula apresenta duas ligações de hidrogênio intramoleculares, de força média (distâncias 0-0 2,558(3) e 2,674(4) $\AA$ ). 0 empacotamento cristalino apresenta duas outras ligações de hidrogênio intermoleculares, mais fracas, sendo feitas com as moléculas geradas pelo espelho c (distâncias 0-0 2,830(3) e 2,992(3) $\AA$ ). 0 efeito destas ligações intermoleculares é o da formação de cadeias ao longo da direção [101]. 


\section{ABSTRACT}

Initially, some considerations about X-ray diffraction, its laws, the factors for the correction of experimental data, a revision of the main Direct Methods for structure resolution, and comments on the refinement of the resulting structure are presented

Next, the structure determination of the compound 5,4'-dihydroxy-3'-5'dimethoxy-6,7(2",2")dimethylpyran)flavone is described

The compound is isolated from plants of the species Neuroputia paraenesis and has the molecular formula $\mathrm{C}_{22} \mathrm{H}_{20} \mathrm{O}_{7}$. It crystallizes in the monoclinic system, space group $\mathrm{C} 2 / \mathrm{c}$, with the following unit cell parameters: $\mathrm{a}=13.651(1), \mathrm{b}=23.428(2), \mathrm{c}=13.725(1) \AA ; \beta=$ $119.528(4)^{\circ}, V=3819.6(5) \mathrm{A}^{3}, D_{c}=1.366 \mathrm{~g} \mathrm{~cm}^{-3}$ e $Z=8$ molecules per unitcell.

The structure was solved applying Direct Methods. The final disagreement indices are: $R=0.0509, R_{w}=0.0530$ for 1743 reflections with $I \geq 3 \sigma(I)$ and $R_{a l l}=0.157$.

The structure was refined applying Fourier difference calculations and full matrix least squares methods

The molecule shows two intramolecular hydrogen bonds of medium strength (distances $0-02.558(3)$ and $2.674(4) \AA$ ). The crystal packing shows also two weaker hydrogen bonds; these are formed between the molecules generated by the $\mathrm{c}$ mirror (distances O-O 2.830(3) and $2.992(3) \AA$ ). The result of these intermolecular hydrogen bonds is the formation of chains in [101] direction. 


\section{INTRODUÇÃO}

A classe de compostos denominada flavonóides é um conjunto de substâncias de ocorrência natural, compreendendo vários tipos de compostos.

O termo flavonóide foi aplicado por Geissman e Hinreiner [1952], para abranger todos os compostos, cuja estrutura está baseada sobre a molécula de flavona, que é a mais abundânte desta classe.

A figura 1 mostra a estrutura de uma molécula de flavona

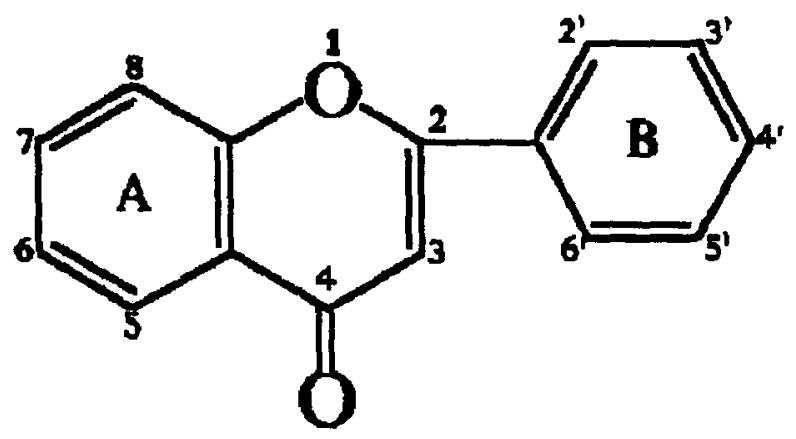

Figura 1 Representação estrutural de uma molécula de Flavona, com a numeração típica usada nos flavanóides.

Observando-se a figura 1 , verifica-se que a molécula consiste de dois anéis benzênicos (A e B) unidos por uma ligação de três carbonos formada em um anel $\gamma$-pirona.

Para exemplificar a classe dos flavonóides a figura 2 representa alguns dos membros desta classe: 


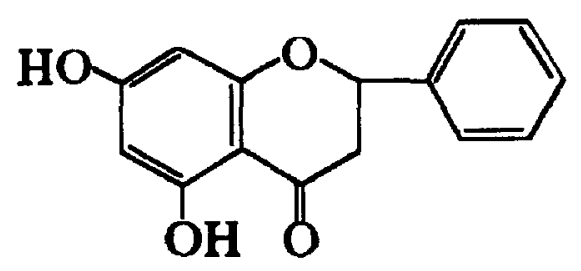

(a)

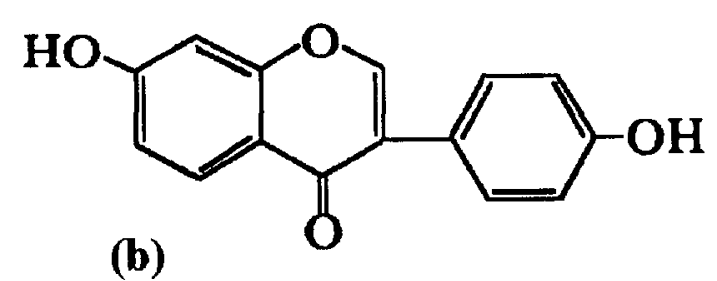<smiles>O=c1c(O)c(-c2ccc(O)c(O)c2)oc2cc(O)cc(O)c12</smiles>

(c)

Figura 2 - Representação estrutural de alguns compostos da classe dos flavonóides. (a) flavanona; (b) isoflavona; (c) flavonol.

As flavonas são abundantemente encontradas em plantas superiores, atuando no crescimento das plantas e sendo também responsáveis pela sua pigmentação, que proporciona uma faixa de tonalidade que abrange do amarelo claro ao laranja, localizado-se principalmente em flores, poléns,e frutos. Embora não sejam sintetizadas pelos animais, as flavonas estão presentes nas asas de algumas borboletas, provenientes de sua dieta alimentar [Morris e Thomson, 1963].

A razão dos diversos componentes desta classe serem coloridos é devida a eles absorverem luz na região do espectro que se localiza entre 400 e $800 \mathrm{~m} \mu$, e na região do ultravioleta (150 e 400m $\mu$ ) causando excitação dos elétrons na molécula [Goodwin, 1965].

Uma outra característica da classe dos flavanóides, e em particular das flavonas, é que membros desta classe são associados com uma grande variedade de atividades farmacológicas, atividades estas que dependem da estrutura e orientação dos vários grupos na molécula.

Desta forma as flavonas podem ser usadas no tratamento de doenças. Por exemplo sabe-se que pequenas quantidades da substância podem agir como estimulantes 
cardíacos, algumas possuem propriedades antibacterianas; outras são agentes antialérgicos; ainda podem ser citadas outras propriedades tais como: febrífugas, calmantes, ictiotóxicas, podendo ainda inibir ou estimular certos sistemas de enzimas [Ikan, 1991].

Um exemplo de uso farmacológico é a substância $3^{\prime}, 4^{\prime}, 5^{\prime}, 8$-tetrametoxi- 5hidroxi-7,6-(2",2", dimeltilpirano)-flavona, que tem seu uso relatado na literatura na medicina popular possuindo atividades antitérmicas e calmantes entre outras [Arruda e outros, 1993].

Esta dissertação tem por objetivo descrever a determinação e a estrutura final obtida de uma flavona de fórmula molecular $\mathrm{C}_{22} \mathrm{H}_{20} \mathrm{O}_{7}$, a 5,4' -Dihidroxi-3',5'-dimetoxi- 6,7 (2",2"-dimetilpirano)flavona, obtida a partir de plantas da espécie Neoraputia paraensis, vulgarmente conhecidas como capança, catanduva, caporé e pau branco

A substância foi isolada pelo Prof.Dr. Lourivaldo da S. Santos [Santos e outros, 1995] a partir do extrato diclorometânico de folhas e caules através de técnicas cromatográficas, e purificada por cristalização.

Uma vez a estrutura resolvida o estudo posterior será desenvolvido pelo Prof. Lourivaldo da Silva Santos (UFPA), que irá testar as suas atividades antiflamatórias e analgésicas, além de visar a correlação entre a estutura química e atividade farmacológica. 


\section{CAPÍTULO I REVISÃO TEÓRICA}

\section{1 - INTRODUÇÃO}

Neste capítulo apresenta-se uma revisão sobre os fundamentos teóricos da cristalografia de determinação de estruturas cristalinas e moleculares. Partindo inicialmente da natureza dos raios- $X$, são feitas considerações sobre os tipos de espalhamento destes pela matéria, apresentando-se a seguir as leis que descrevem as condições de difração

São abordados também tópicos referentes às correções feitas sobre os dados de intensidade observada das reflexões dos planos cristalinos.

A abordagem do problema das fases é feita na sua relação com os métodos diretos de resolução de estrutura.

No refinamento da estrutura dá-se ênfase ao método de mínimos quadrados e síntese de Fourier diferença.

\section{1 - ORIGEM DA PRODUÇÃO DE RAIOS-X [CULLITY, 1967]}

O estudo de uma estrutura cristalina está baseado no fenômeno de difração dos raios- $\mathrm{X}$, causado pela sua interação com elétrons dos átomos.

No espectro eletromagnético, os raios-X localizam-se entre a luz ultravioleta e a radiação gama, compreendendo uma faixa de comprimento de onda de 0,1 a $100 \AA$, sendo o intervalo mais útil para os fins analíticos o de 0,50 a $2,5 \AA$.

Os raios-X são produzidos quando elétrons com alta velocidade colidem com um metal alvo. Basicamente um tubo de produção de raios-X é constituído por uma fonte de 
origem de elétrons (catodo), um acelerador de alta voltagem, e um metal alvo (anodo). Estes tubos contém dois eletrodos, um ânodo (metal alvo), mantido a um potencial baixo e um cátodo mantido a um potencial negaivo da ordem de 30.000 a $50.000 \mathrm{~V}$.

Quanto a sua natureza, os raios-X, são portanto ondas eletromagnéticas, que possuem associadas um campo elétrico (E) e um campo magnético (H). Isto pode ser representado utilizando-se a figura 3 , onde um feixe de raios-X monocromático caminhando na direção do eixo $\mathbf{x}$ tem associados, um campo elétrico (E) na direção do eixo y e um campo magnético $(\mathrm{H})$ na direção do eixo $\mathrm{z}$.

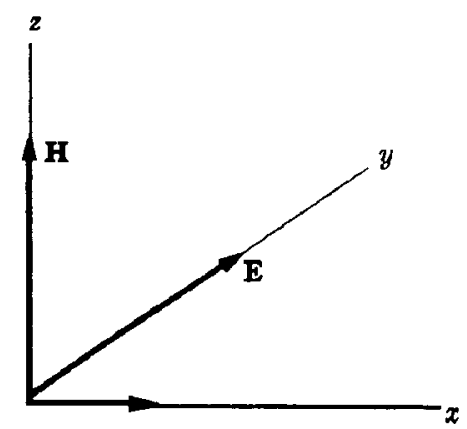

Figura 3- Campos elétrico e magnético associados com o movimento da onda de raios-X na direção do eixo x [Cullity, 1967].

Se o campo elétrico é confinado ao plano yx, como as ondas caminham nesta direção, a onda é plano polarizada.

Uma onda plano-polarizada (E) não é constante com o tempo, mas varia de um máximo na direção de ty e atravessa para um outro máximo na direção de -y, e se assumirmos ambas variações como senoidais, podemos expressar a variação na equação

$$
E=A \operatorname{sen} 2 \pi\left(\frac{x}{\lambda}-v\right)
$$

onde: $A=$ amplitude da onda; $\lambda=$ comprimento de onda; $v=$ frequência; $x=$ espaço percorrido $\mathrm{e} \mathrm{t}=$ tempo 
Radiações eletromagnéticas, como por exemplo um feixe de raios-X, carregam energia, e a razão do fluxo desta energia através de unidade de área perpendicular a direção do movimento é chamado de Intensidade (I).

\section{2 - INTERAÇÃO DOS RAIOS-X COM A MATÉRIA}

\subsection{1 - ESPALHAMENTO POR UM ELÉTRON [CULLITY, 1967]}

Quando feixes de raios-X interagem com a matéria ocorrem dois processos de espalhamento: o Coerente ou Thomson e o espalhamento Incoerente ou Compton.

O espalhamento Coerente, ocorre quando a radiação-X atinge o elétron livre e o raio espalhado por este elétron possui a mesma frequência e comprimento de onda do raio incidente, apenas diferenciando na fase por $180^{\circ}$, portanto todos os raios espalhados por um único elétron tem a mesma relação de fase do feixe incidente

Uma vez que os raios-X são espalhados em todas a direções pelo único elétron a intensidade dos raios espalhados dependerão do ângulo de espalhamento. Esta teoria eletromagnética desenvolvida por J.J Thomson mostra que:

$$
I=I_{0} \frac{e^{4}}{r^{2} m^{2} c^{4}}\left(\frac{1+\cos ^{2} 2 \theta}{2}\right)
$$

onde: $\theta$ é o ângulo de espalhamento;

$\mathrm{I}_{0}$ é a intensidade do feixe incidente;

c é a velocidade da luz, $m$ é a massa do elétron;

r é a distância total do espalhamento;

e é carga do elétron ;

$\left(1+\cos ^{2} 2 \theta\right)=$ fator de polarização dos raios-X espalhados. 
A outra maneira em que o elétron pode espalhar os raios-X, é manifestada no efeito Compton ou espalhamento incoerente, que ocorre na colisão de um fóton com um elétron livre, tendo o fóton energia $h v_{1}$, Neste caso de colisão a colisão é elástica sendo essencialmente um efeito semelhante ao choque de duas bolas de bilhar (figura 4). O elétron é colidido pelo fóton e este é desviado de um ângulo de $2 \theta$, com uma energia $h v_{2}$, e alguma parte da energia inicial do fóton é transferido na forma de energia cinética para o elétron.

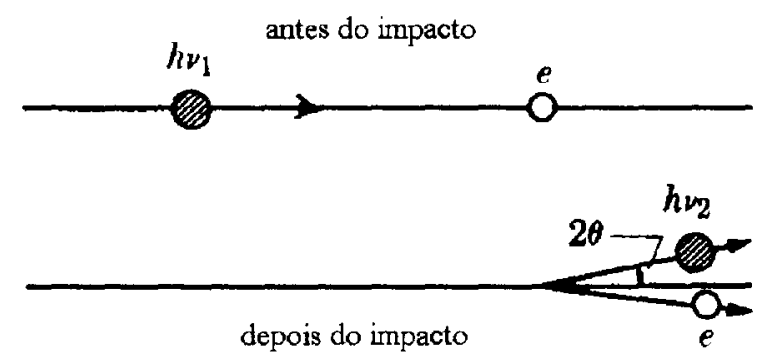

Figura 4 - Colisão elástica entre fóton e elétron (Demonstração do Efeito Compton)

O comprimento de onda $\left(\lambda_{2}\right)$ da radiação espalhada é então ligeiramente maior que o comprimento de onda $\left(\lambda_{1}\right)$ do raio incidente e a magnitude da variação é dada pela expressão:

$$
\Delta \lambda(\AA)=\lambda_{2}-\lambda_{1}=0,0243(1-\cos 2 \theta)
$$

O aumento no comprimento de onda depende somente do ângulo de espalhamento e varia de zero na direção $\left(2 \theta=0^{\circ}\right)$ a $0,05 \AA$ na direção extrema $\left(2 \theta=180^{\circ}\right)$.

Então, a característica principal na radiação Compton é que sua fase não tem relação fixada com a fase do raio incidente, sendo por essa razão também conhecida como radiação incoerente. Este tipo de espalhamento é ignorado em experimentos de difração, causando apenas um efeito indesejável de escurecimento do fundo [Cullity, 1967] 


\subsection{2 - ESPALHAMENTO POR UM ÁTOMO}

Quando um feixe de raios-X encontra um átomo, cada elétron espalha a radiação coerente ou Thomson de acordo com a equação 2 [Cullity, 1967].

Os núcleos deveriam tomar parte no espalhamento uma vez que possuem cargas e deveriam ser capazes de oscilar sob a influência de um feixe de raios- $X$ incidente, mas isto não ocorre devido a sua enorme massa em relação ao elétron, portanto não pode oscilar numa apreciável extensão. A massa do próton é cerca de 2000 vezes a massa do elétron, e por esta razão o próton é muito pesado para ser um emissor secundário, uma vez que a equação 2 tem dependência do inverso do quadrado da massa da partícula espalhadora

Pode-se concluir que as ondas espalhadas por um átomo são simplesmente a soma das ondas espalhadas pelos seus elétrons constituintes, notando que os elétrons de um átomo estão situados em diferentes pontos no espaço, e que isto introduz diferenças nas fases, entre as ondas espalhadas por diferentes elétrons, assim por exemplo, se o espalhamento está ocorrendo na direção de $2 \theta=0$, as ondas espalhadas por todos os elétrons estarão em fase e as amplitudes de todas as ondas espalhadas podem ser somadas diretamente.

Define-se assim, uma quantidade chamada de fator de espalhamento $\left(f_{0}\right)$, que descreve a eficiência de espalhamento de um átomo qualquer, em uma dada direção, segundo a equação:

$$
f_{\mathrm{o}}=\frac{\text { Amplitude da onda espalhada por um atomo }}{\text { Amplitude da onda espalhada por um eletron }}
$$

onde, $f_{0}$ é expresso em termos de poder de espalhamento de um número equivalente de elétrons localizados na posição do núcleo atômico. 
Assumindo os átomos como esféricos, devido à parcial interferência que ocorre entre os espalhamentos pelos diferentes elétrons, o poder de espalhamento dos átomos é função do tipo de átomo, e de $\operatorname{sen} \theta / \lambda$, ou seja, do ângulo de incidência do feixe e do comprimento de onda, não dependendo portanto da sua posição na cela unitária do cristal.

A curva de variação do fator de espalhamento [Stout e Jensen, 1989], por exemplo para o átomo de Carbono, em função de $(\operatorname{sen} \theta / \lambda)$ está representada na figura 5 .

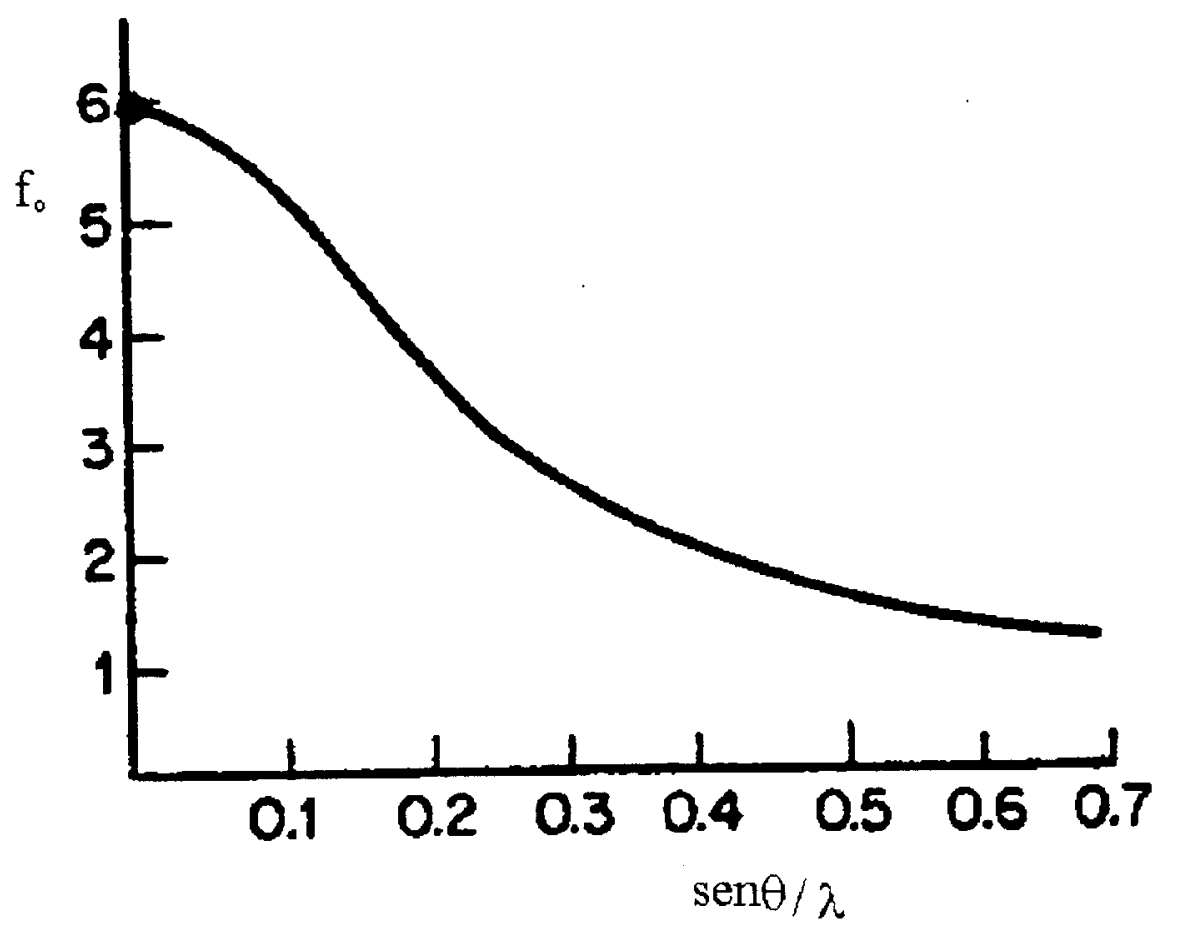

Figura 5- Curva do fator de espalhamento para o átomo de carbono.

Observando que em $\operatorname{sen} \theta=0$, o fator de espalhamento atômico para o carbono é igual a 6, correspondendo ao seu número atômico, a medida em que se aumenta o valor de $\operatorname{sen} \theta / \lambda$ o valor de $f_{o}$ decai, devido aos raios- $X$ espalhados em uma parte do átomo estarem fora de fase com aqueles espalhados em outra parte da nuvem eletrônica, portanto a variação do fator de espalhamento é uma consequência do tamanho finito do átomo. 


\subsection{3- ESPALHAMENTO POR UMA CELA UNITÁRIA}

De acordo com as diferentes eficiências com que os diversos átomos presentes em uma cela unitária difratam os raios-X, segundo o espalhamento coerente, as ondas difratadas tem diferentes intensidade ou seja diferentes amplitudes.

Seja a representação de uma cela unitária representada na figura 6:

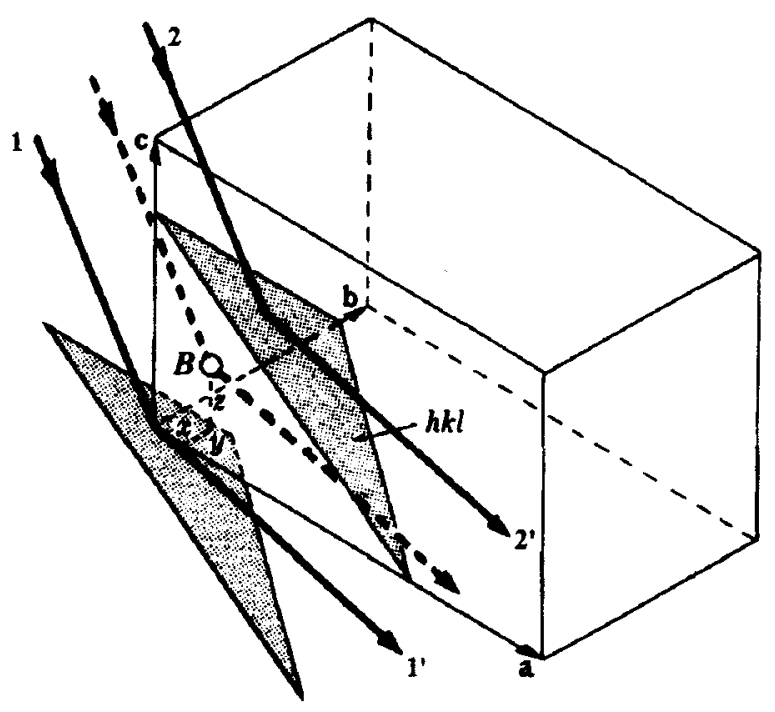

Figura 6 - Representação tridimensional de uma cela unitária genérica [Cullity,1967].

Estas duas ondas incidentes, representadas pelos números 1 e 2 , podem diferir em fase e amplitude, se o átomo B e o átomo na origem forem de espécie diferentes, então pode-se representar estas duas ondas em uma forma vetorial como ilustrado na figura $7 \mathrm{a}$.

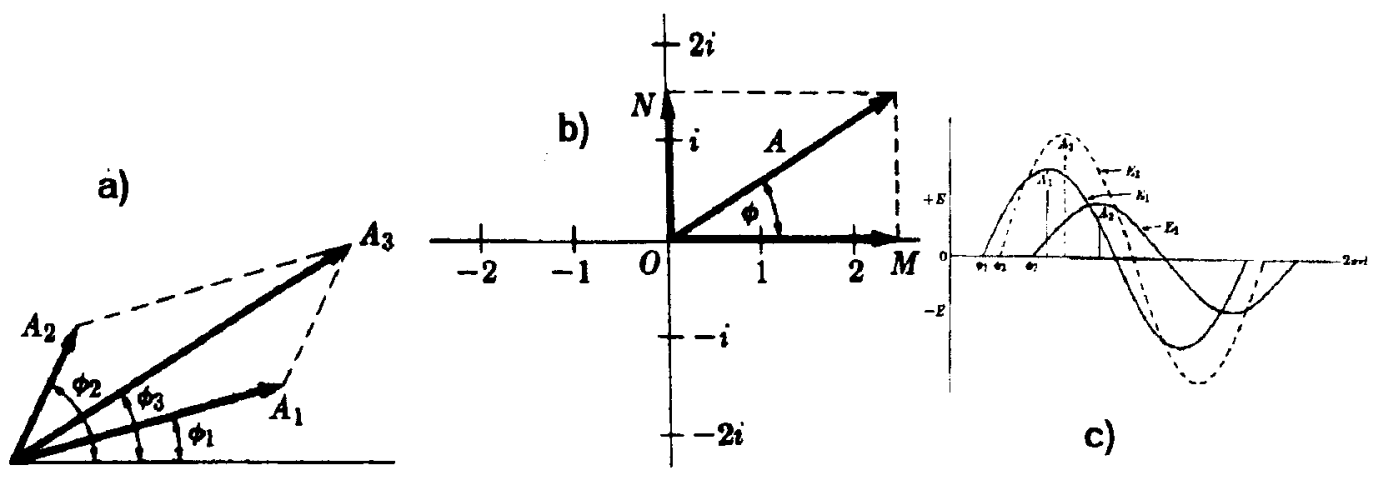

Figura 7 - Adição de ondas: a) tratamento vetorial; b) tratamento analítico; c)ondas senoidais. 
$\mathrm{Na}$ figura $7 \mathrm{a}, \mathrm{A}_{1}$ é a amplitude da onda $1, \phi_{1}$ a sua fase, $A_{2}$ e $\phi_{2}$ amplitude e fase da onda 2 e $A_{3}$ e $\phi_{3}$ são a amplitude e a fase resultantes, encontradas pela adição de vetores.

Utilizando-se um tratamento analítico, no qual números complexos são utilizados para representar os vetores, podemos construir a figura $7 \mathrm{~b}$. A figura $7 \mathrm{c}$ é uma representação convencional da adição de ondas senoidais.

A partir da figura 7 pode-se construir uma expressão analítica para onda na forma de número complexo, $\mathrm{Ae}^{\mathrm{ix}}$, com $\mathrm{A}$ representando o comprimento do vetor onda (amplitide) e $\phi=\mathrm{ix}=\mathrm{a}$ fase da onda.

Exprimindo em séries de $\mathrm{e}^{\mathrm{ix}}, \cos \mathrm{x}$ e sen $\mathrm{x}$ temos

$$
\begin{aligned}
& e^{i x}=\cos x+i \operatorname{sen} x \\
& A e^{i \phi}=A \cos \phi+A i \operatorname{sen} \phi
\end{aligned}
$$

Como a intensidade de uma onda é proporcional ao quadrado de sua amplitude necessita-se encontrar uma expressão para $A^{2}$ ( o quadrado do valor absoluto do vetor onda); desta forma multiplicando-se a expressão 6 pelo complexo conjugado tem-se:

$$
\left|A e^{i \varnothing}\right|^{2}=A e^{i \phi} A e^{-i \phi}=A^{2}
$$

Como a amplitude de cada onda é dada pela soma do valor apropriado de $f_{0}, o$ espalhamento atômico, em função dos valores de $\operatorname{sen} \theta / \lambda$ envolvidos na reflexão, a fase de cada átomo sera dada por:

$$
\phi=2 \pi(\mathrm{hx}+\mathrm{ky}+\mathrm{lz})
$$

onde $\mathrm{h}, \mathrm{k}, \mathrm{l}$ são os indices de Miller dos planos de reflexão considerados, e x, y ,z são as coordenadas atômicas.

Expressando a onda espalhada por cada átomo, em forma exponencial complexa, tem-se: 


$$
A e^{i \phi}=f e^{2 \pi i(h x+k y+l z)}
$$

A onda resultante, espalhada por todos os átomos na cela unitária é denominada Fator de Estrutura, designado pelo símbolo F; este valor é obtido pela soma de todas as ondas espalhadas por átomos individuais [Stout e Jensen,1989], a equação 9 passa a ter a forma:

$$
\mathrm{F}_{\mathrm{bk} l}=\sum_{1}^{N} \mathrm{f}_{\mathrm{j}} \mathrm{e}^{2 \pi i(\mathrm{kx}+\mathrm{ky}+\mathrm{k})}
$$

A expressão 10 representa o fator de estrutura, de modo a fornecer acesso direto às três coordenadas no espaço direto $(\mathrm{xyz})$, e aos três índices no espaço recíproco $(\mathrm{hk} l)$, Pode-se então representar a equação 10 em uma outra notação, indicando os três índices do vetor no espaço recíproco por $\mathbf{h}$, e as três coordenadas no espaço direto pelo vetor $\mathbf{r}$, e definindo o produto escalar destes dois vetores obtem-se:

$$
\text { h.r }=h x+k y+l z
$$

Então a expressão 10 pode ser reescrita como:

$$
F_{h}=\sum f_{j} e^{2 \pi i(h \cdot r)}
$$

ou ainda:

$$
F_{h}=\Sigma f_{j}[\cos 2 \pi(\text { h.r })+i \operatorname{sen} 2 \pi(\text { h.r })]
$$

\section{3 - A REDE RECÍPROCA}

Redes reciprocas são redes triperiódicas, derivadas da rede no espaço direto.

Assumindo uma rede no espaço direto e uma cela unitária definida pelos vetores $\mathbf{a}_{1}, \mathbf{a}_{2}, \mathbf{a}_{3}$, a rede recíproca terá uma cela unitária definida pelos vetores $\mathbf{b}_{1}, \mathbf{b}_{2}, \mathbf{b}_{3}$ com: 


$$
\begin{aligned}
& \mathbf{b}_{1}=1 / V\left(\mathbf{a}_{2} \times \mathbf{a}_{3}\right) \\
& \mathbf{b}_{2}=1 / V\left(\mathbf{a}_{1} \times \mathbf{a}_{3}\right) \\
& \mathbf{b}_{3}=1 / V\left(\mathbf{a}_{1} \times \mathbf{a}_{2}\right)
\end{aligned}
$$

onde, V é o volume da cela unitária [Cullity,1967].

Os pontos constituintes da rede recíproca descrevem completamente o cristal, de tal forma, que cada ponto da rede recíproca está relacionado com um conjunto de planos na rede direta e mostra a orientação e o espaçamento daquele conjunto de planos, como mostrado na figura 8.

Quanto as propriedades da rede recíproca verifica-se que:

a- o vetor $\mathbf{H}_{\mathbf{h k} \text {, }}$ partindo da origem da rede recíproca para algum ponto na mesma, com as coordenadas hkl, é perpendicular ao plano da rede cristalina com índices de Miller hkl, este vetor é dado em têrmos de suas coordenadas pela expressão:

$$
\mathbf{H}_{\mathrm{hk} l}=\mathrm{h} \mathbf{b}_{1}+\mathrm{k} \mathbf{b}_{2}+l \mathbf{b}_{3}
$$

b- $\mathrm{O}$ comprimento do vetor $\mathbf{H}_{\mathrm{hkl}}$ é igual ao recíproco do espaçamento dos planos(hkl) ou:

$$
\left|\mathbf{H}_{\mathrm{hk} l}\right|=1 / \mathrm{d}_{\mathrm{hk} l}
$$

onde $\mathrm{d}_{\mathrm{hk} l}$ é o espaçamento interplanar entre os planos vizinhos da família (hk $l$ ).

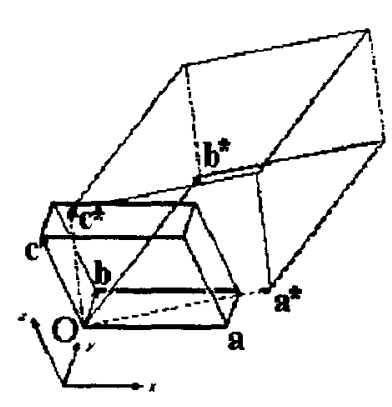

$\mathbf{a}$

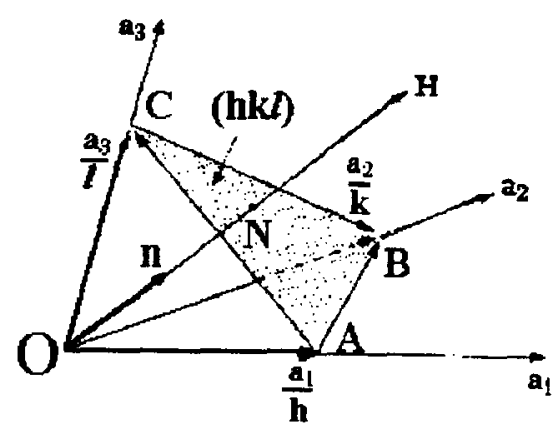

b

Figura 8 - Espaço Recíproco. a) representação dos eixos. b) relação entre o vetor H e 0 plano hkl. 


\section{4 - A LEI DE BRAGG}

Em 1912 W. L. Bragg deduziu uma expressão simples para tratar o fenômeno de difração originado pelos planos da rede cristalina [Stout e Jensen,1989].

$\mathrm{Na}$ figura 9 está representada uma família de planos $\mathrm{P}_{1}$ e $\mathrm{P}_{2}$ com espaçamento interplanar $\left(\mathrm{d}_{\mathrm{hk} l}\right)$, e com orientação, relativa ao feixe de raios-X incidente, expressa pelo ângulo de incidência $\theta$.

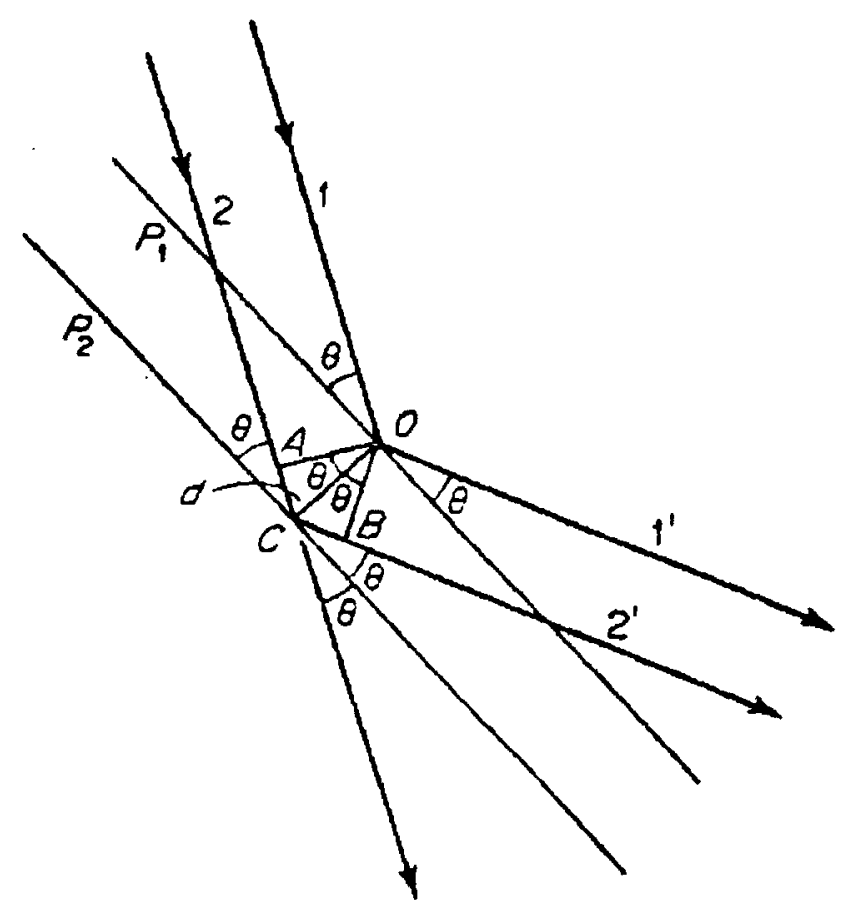

Figura 9 - Construção apresentando as condições de difração.

$\mathrm{Na}$ figura 9, 1 e 2 representam os feixes de raios-X incidentes, 1'e 2', correspondem aos feixes difratados, $\mathrm{O}$ e $\mathrm{C}$ são as posições dos elétrons no plano, e $\theta$ é o ângulo que os raios 1 e 2 fazem com os planos $P_{1}$ e $P_{2}$.

Pela figura 9 verifica-se que: 


$$
\angle \mathrm{AOC}=\angle \mathrm{BOC}=\theta
$$

então se :

$$
\mathrm{AC}=\mathrm{BC}
$$

$\mathrm{e}$

$$
\mathrm{AC}+\mathrm{CB}(=2 \mathrm{AC})
$$

ou seja um número inteiro de comprimento de onda $(\lambda)$, resultando em interferências construtivas (máximos de difração).

Pode-se então expressar a lei da difração da seguinte forma:

$$
2 \mathrm{AC}=\mathrm{n} \lambda
$$

onde $\mathrm{n}$ é um número inteiro.e representa a ordem de difração. Entretanto como, por definição, $\mathrm{AC} / \mathrm{d} \equiv \operatorname{sen} \theta$, substituindo na expressão 22 , obtem-se finalmente:

$$
\mathrm{n} \lambda=2 \mathrm{~d}_{\mathrm{hk} l} \operatorname{sen} \theta
$$

A equação 23 chamada de Lei de Bragg mostra que a difração ocorre somente a ângulos especiais, e descreve a condição para a difração dos vários raios incidentes, fazendo um ângulo $\theta$ com planos fixados do cristal. No caso da reflexão (difração) ocorrer, para esses planos, geram-se os raios refletidos com desvio de $2 \theta$, em relação aos raios incidentes, somente quando $\theta$ e $\lambda$ são compatíveis com a distância interplanar $\left(\mathrm{d}_{\mathrm{hk} l}\right)$, aí então existe reforço das ondas refletidas.

\section{5 - AS EQUAÇÕES DE LAUE}

As equações de Laue exprimem a condição necessária para que as ondas refletidas, por diferentes átomos presentes no cristal, estejam em fase e originem um máximo de interferência construtiva [Borges,1980].

As equações de Laue podem ser obtidas a partir da figura 10 
Para que a interferência dos raios difratados resulte em um máximo de difração, a diferença de percurso destes deve ser igual a um número inteiro $\left(\mathrm{H}_{1}\right)$ de comprimento de onda, desta forma tem-se:

$$
A_{1} C-A_{2} B=H_{1} \lambda
$$

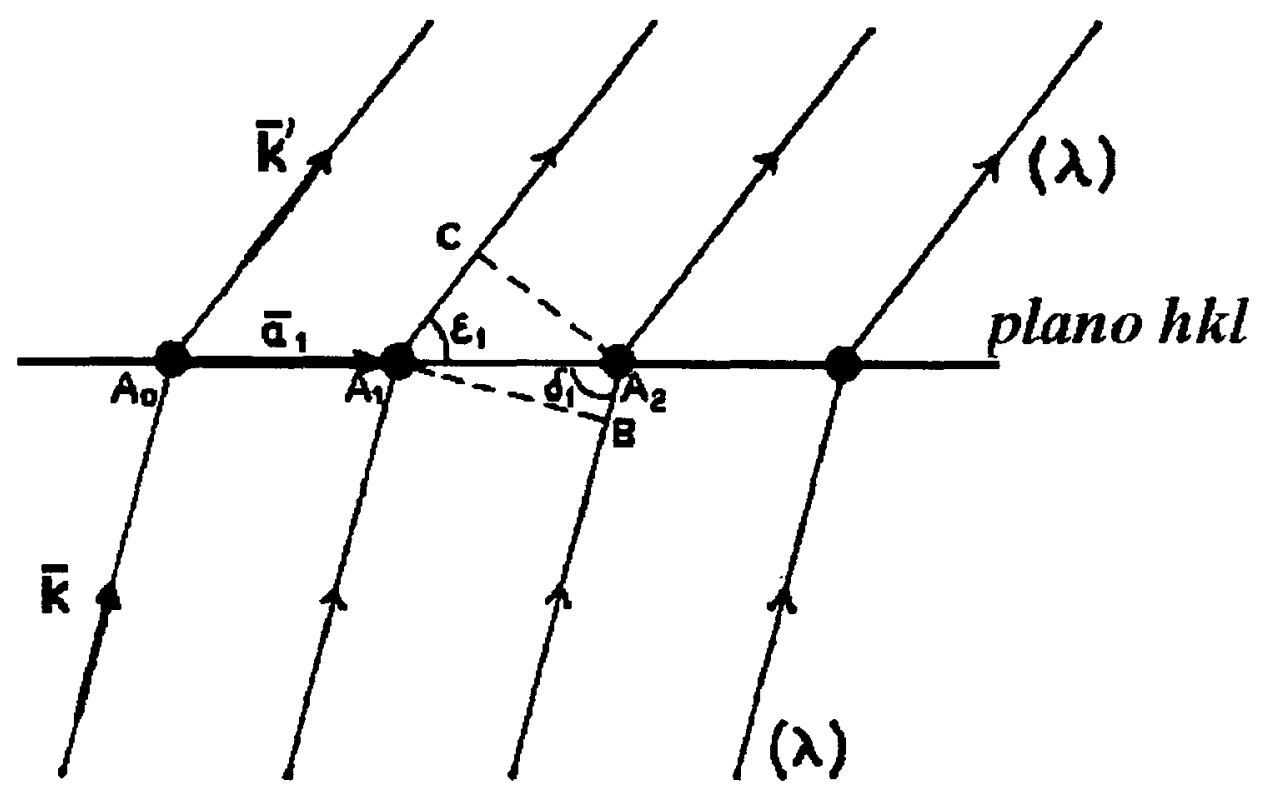

Figura 10- Diferença de percurso entre os raios incidentes e difratados

Da geometria da figura 10 verifica-se que:

$$
A_{1} C=\mathbf{a}_{1} \cos \varepsilon_{1}
$$

$\mathrm{A}_{2} \mathbf{B}=\mathbf{a}_{1} \cos \delta_{1}$

Substituindo as equações 25 e 26 na expressão 24 obtem-se:

$$
\mathbf{a}_{1}\left(\cos \varepsilon_{1}-\cos \delta_{1}\right)=\mathrm{H}_{1} \lambda
$$

Expressando vetorialmente as equações 25 e 26

$$
\begin{aligned}
& A_{1} C=a_{1} \cdot k \\
& A_{2} B=a_{1} . k
\end{aligned}
$$


onde, $\mathbf{a}_{1}$ é o vetor reticular definindo a fila; $\mathbf{k}$ e k' são vetores unitários na direção dos raios incidentes e difratados, e substituindo as expressões 28 e 29 em 27 tem-se finalmente:

$$
\mathbf{a}_{1} \cdot\left(\mathbf{k}^{\prime}-\mathbf{k}\right)=\mathrm{H}_{1} \lambda
$$

que descreve a difração para uma estrutura unidimensional.

Considerando agora o caso real, para uma estrutura cristalina possuindo caráter triperiódico, a condição de difração dos raios-X monocromáticos, é que o fenômeno de difração ocorrerá somente nas direções que satisfaçam o sistema de três equações:

$$
\begin{aligned}
& \mathbf{a}_{1}\left(\cos \varepsilon_{1}-\cos \delta_{1}\right)=H_{1} \lambda \\
& \mathbf{a}_{2}\left(\cos \varepsilon_{2}-\cos \delta_{2}\right)=H_{2} \lambda \\
& \mathbf{a}_{3}\left(\cos \varepsilon_{3}-\cos \delta_{3}\right)=H_{3} \lambda
\end{aligned}
$$

Expressando-se vetorialmente as equações de 31 a 33 obtem-se:

$$
\mathbf{a}_{\mathbf{i}}\left(\mathbf{k}^{\prime}-\mathbf{k}\right)=\mathrm{H}_{\mathrm{i}} \lambda
$$

onde $\mathrm{i}=1,2,3$ (espaço tridimensional)

Definindo-se a direção de propagação da onda [Vainshtein, 1981] pelo vetor $\mathbf{k}$ cujo módulo é dado por:

$$
|\mathbf{k}|=2 \pi / \lambda
$$

e, utilizando a equação 34 , pode-se escrever as equações de Laue de uma outra forma:

$$
\begin{array}{lll}
\mathbf{a}_{1}\left(\mathbf{k}^{\prime}-\mathbf{k}\right)=2 \pi \mathrm{h} & \text { ou } & \mathbf{a}_{1} \mathbf{S}=\mathrm{h} \\
\mathbf{a}_{2}\left(\mathbf{k}^{\prime}-\mathbf{k}\right)=2 \pi \mathrm{k} & \text { ou } & \mathbf{a}_{2} \mathbf{S}=\mathbf{k} \\
\mathbf{a}_{3}\left(\mathbf{k}^{\prime}-\mathbf{k}\right)=2 \pi l & \text { ou } & \mathbf{a}_{3} \mathbf{S}=l
\end{array}
$$

chegando-se então à descrição da difração por um cristal, com a direção dos raios espalhados definida por:

$$
\mathbf{S}=\mathbf{H}_{\mathrm{hkl}}=\mathrm{h} \mathbf{a}^{*}+\mathrm{k} \mathbf{b}^{*}+l \mathbf{c}^{*}
$$




\subsection{1- EQUIVALÊNCIA DAS EQUAÇ̃̃ES DE LAUE E A LEI DE BRAGG}

As equações de Laue e a da lei de Bragg fornecem, sob diferentes pontos de vista, as condições necessárias para ocorrência de máximos de difração, ou seja, a condição de difração de raios- $\mathrm{X}$ por um cristal pode ser expressa pela equação de Bragg ou em têrmos das equações de Laue [Borges, 1980].

Como descrito anteriormente, as equações de Laue são expressas pela equação 34 que de forma simplificada pode ser escrita como:

$$
\left(\mathbf{k}^{\prime}-\mathbf{k}\right)=\lambda \mathbf{g}
$$

onde $\mathrm{g}$ é o vetor da rede recíproca; passando a ter a forma:

$$
\mathbf{a}_{\mathbf{i}} \cdot \mathbf{g}=\mathbf{H}_{\mathbf{i}} \quad(\mathrm{i}=1,2,3)
$$

Escolhendo-se para referencial cristalográfico os três eixos definidos pelos vetores $\mathbf{a}_{\mathbf{i}}$ e exprimindo o vetor $\mathbf{g}$ em termos de rede recíproca com constante $\mathrm{k}=1$, obtemse:

$$
\begin{aligned}
& \mathbf{g}=g_{1} \mathbf{a}_{\mathbf{1}}{ }^{*}+g_{2} \mathbf{a}_{\mathbf{2}}{ }^{*}+g_{3} \mathbf{a}_{\mathbf{3}}{ }^{*} \\
& \mathbf{g}=\mathbf{g}_{\mathbf{i}} \mathbf{a}_{\mathbf{i}}{ }^{*}
\end{aligned}
$$

sendo :

$$
\mathbf{a}_{1}^{*}=\frac{\mathbf{a}_{2} \times \mathbf{a}_{3}}{\mathbf{a}_{1} \bullet \mathbf{a}_{2} \times \mathbf{a}_{3}}, \ldots \text { etc. }
$$

Usando esta expressão e inserindo-a na expressão 41 tem-se:

$$
\mathbf{a}_{\mathbf{i}} \cdot \mathbf{g}=\mathbf{a}_{\mathbf{i}}\left(\mathbf{g}_{\mathbf{i}} \cdot \mathbf{a}_{\mathbf{i}}{ }^{*}\right)
$$

como:

$$
a_{i} \cdot a_{i}^{*}=a_{i} \frac{a_{j} \times a_{k}}{a_{i} \cdot a_{j} \times a_{k}}=\frac{V}{V}=1
$$

e, 


$$
a_{i-a_{j}}{ }^{*}=a_{i} \frac{a_{k} \times a_{i}}{a_{i} \bullet a_{j} \times a_{k}}=\frac{0}{V}=0 ; \text { etc... }
$$

resulta

$$
\mathbf{a}_{\mathbf{i}} \cdot \mathbf{g}=\mathbf{g}_{\mathbf{i}}
$$

Substituindo 48 em 44 :

$$
\mathbf{g}_{\mathbf{i}}=\mathbf{H}_{\mathbf{i}}
$$

Como o vetor g é descrito pela equação 43 obtem-se:

$$
\mathbf{g}=\mathrm{H}_{1} \cdot \mathbf{a}_{1}{ }^{*}+\mathrm{H}_{2} \cdot \mathbf{a}_{2}{ }^{*}+\mathrm{H}_{3} \cdot \mathbf{a}_{3}{ }^{*}
$$

e pode-se concluir que o vetor $\mathrm{g}$ é um vetor normal aos planos reticulares (hkl), possuindo uma amplitude igual ao recíproco da distância reticular dos planos $\left(\mathrm{d}_{\mathrm{hk} l}{ }\right)$.

Tendo-se em conta que o vetor $\lambda \mathbf{g}$, por ser igual a $\left(\mathbf{k}^{\prime}-\mathbf{k}\right)$, então existe no plano os vetores $\mathbf{k}$ e $\mathbf{k}^{\prime}$, pode-se verificar algumas das propriedades:

a- Os três vetores $\mathbf{k}, \mathbf{k}^{\prime}$ (direções do raio incidente, difratado) e $\mathbf{g}$ (direção da normal aos planos difratores $(\mathrm{hk} l))$ são coplanares.

b- Dado que $|\mathbf{k}|=\left|\mathbf{k}^{\prime}\right|$, o vetor $\mathbf{g}$ bissecta o ângulo definido pelos dois vetores como na figura 11 , isto é, $\theta=\theta^{\prime} \quad\left(\mathrm{e} \phi=\phi^{\prime}\right)$.

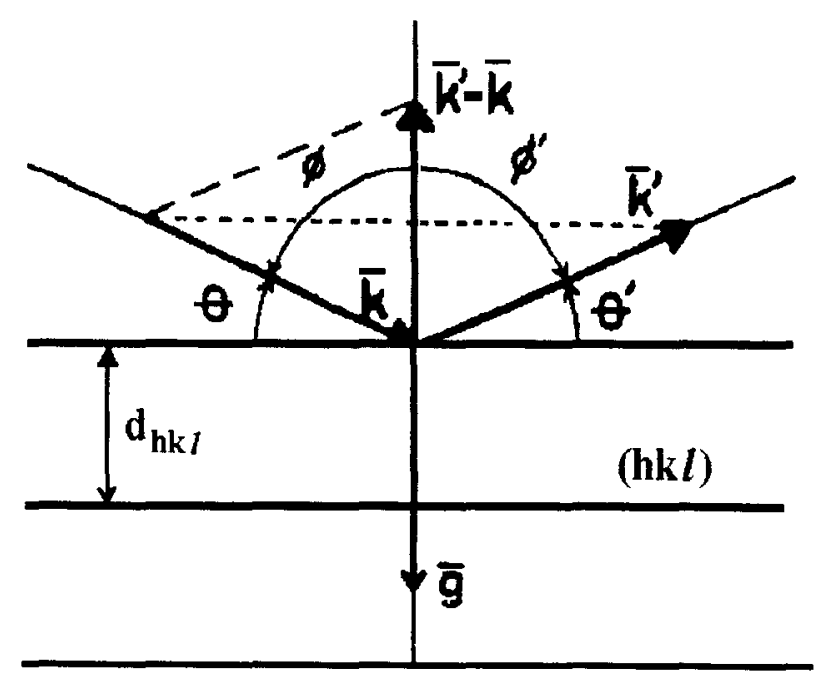

Figura 11- Difração dos raios-X pelos planos cristalinos 
A difração $h k l$ pode ser descrita em termos de uma reflexão nos planos reticulares $(\mathrm{hk} l)$.

A partir da equação 40 e da figura 9 pode-se verificar que:

$$
\left|\mathbf{k}^{\prime}-\mathbf{k}\right|=2 \operatorname{sen} \theta
$$

e

$$
|\mathbf{g}|=1 / \mathrm{d}_{\mathrm{hk} l}
$$

obtendo-se, $\lambda=2 \mathrm{~d}_{\mathrm{hk} k} \mathrm{sen} \theta$, ou de outra forma:

$$
\theta=\operatorname{sen}^{-1}\left(\lambda / 2 \mathrm{~d}_{\mathrm{hk} l}\right)
$$

A expressão acima evidencia que só ocorrerá reflexão de raios-X para os valores de ângulo de incidência igual a $\theta$, ou seja no ângulo de Bragg.

\section{6 - ESFERA DE EWALD}

Como foi visto anteriormente, a condição de difração dos planos de um cristal deve obedecer tanto a lei de Bragg, como as equações de Laue, a partir disto, Ewald em 1921, abrangeu estas duas leis em uma construção geométrica (chamada de esfera de reflexão ou de Ewald) [Azároff, 1968], representada na figura 12.

Pela figura 12 vê-se que a esfera é desenhada centrada no cristal (c) e com raio igual a $1 / \lambda$. A origem da rede recíproca é colocada em um ponto $O$ onde o feixe de raios- $X$, na direção de $\mathrm{AC}$, encontra a esfera. Assim o fenômeno de difração ocorrerá somente quando pontos da rede recíproca toquem a superfície da esfera de Ewald, e se B representa um ponto da rede recíproca, o vetor OB é um vetor do tipo dado pela equação 39. 


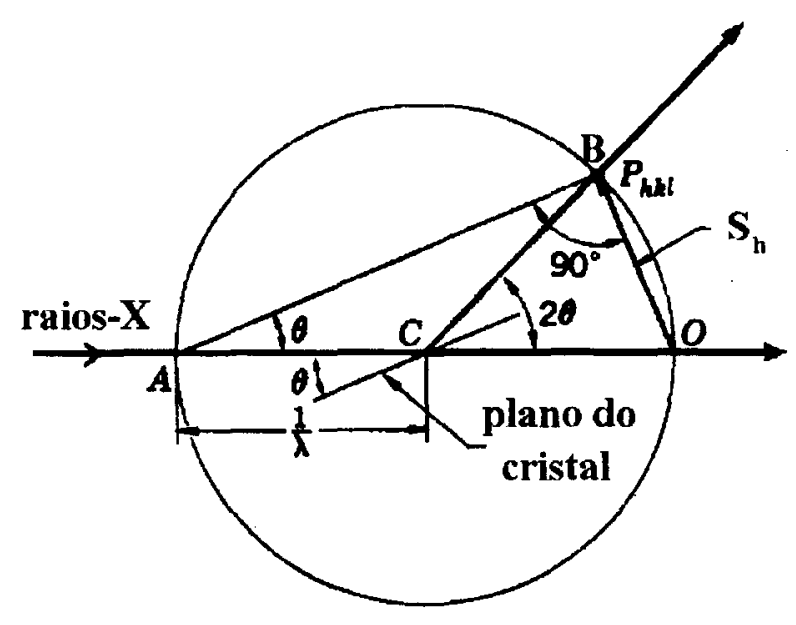

Figura 12 - A Esfera de Ewald ou Esfera de Reflexão

Desta forma para que o plano hkl sofra a difração, o cristal é girado, de modo que os pontos da rede recíproca cortem a esfera de reflexão.

\section{7 -REDUÇÃO DE DADOS}

Em experimentos de difração de raios-X, visando a resolução de estruturas, obtem-se informações a respeito das intensidades (I) das ondas difratadas, que são proporcionais ao quadrado das suas amplitudes, também conhecidas como módulos dos Fatores de Estrutura $\left(\left|F_{\mathrm{hk} /}\right|\right.$ ou $\left.\left|\mathrm{F}_{\mathrm{obs}}\right|\right)$.

No cálculo dos módulos dos fatores de estrutura devem ser considerados alguns fatores que afetam as intensidades, fatores estes, inerentes ao processo experimental [Stout e Jensen, 1989].

Os fatores de estrutura estão relacionados com as intensidades de acordo com a relação: 


$$
\left|F_{h k l}\right|=\left|F_{\text {obs }}\right| \propto \sqrt{I}
$$

ou de foma explícita:

$$
\left|F_{o b s}\right|=\sqrt{\frac{I_{\text {obs }}}{\text { A.Lp }}}
$$

onde, $\mathrm{L}$ é o Fator de Lorentz, p é o fator de polarização e $\mathrm{I}_{\mathrm{obs}}$ é a intensidade medida, e A representa o fator de absorção.

Para o caso da medida ser feita no difratômetro automático CAD-4 ENRAFNONIUS, a intensidade observada é dada por [Enraf-Nonius, 1990]:

$$
\mathrm{I}_{\mathrm{obs}}=\frac{\mathrm{ATN}}{\mathrm{NPI}}(C-R * B)
$$

onde:

$\mathrm{ATN}=$ fator de atenuação

NPI = fator que incorpora a velocidade de varredura

$\mathrm{C}=$ contagem total

$R=$ razão do tempo de varredura do background (radiação de fundo) em relação à contagem total $(R=2$ para a varredura convencional do $C A D-4)$

$\mathrm{B}=$ contagem total do Background

A expressão normalmente aceita para o desvio padrão das intensidades após a correção pelos fator de Lorentz e polarização é dada por:

$$
\sigma\left(\mathrm{F}_{\text {obs }}\right)=\frac{\left.\mathrm{BASE} * \mathrm{ATN} * \sqrt{\left(\mathrm{C}+\mathrm{R}^{2} * B\right.}\right)}{\mathrm{NPI} * \mathrm{Lp}}
$$

onde:

$\mathrm{BASE}=16,49 \% \mathrm{~min}$ no $\operatorname{mad} \delta(1$ 


\subsection{1 - FATORES QUE AFETAM AS INTENSIDADES}

\subsubsection{1 - FATOR DE POLARIZAÇÃO}

O termo trigonométrico fator de polarização $(p)$, aparece em experimentos de difração de raios- $X$, devido a natureza dos raios-X provenientes da fonte não serem polarizados, enquanto que os raios difratados pelo cristal tornam-se polarizados. Isto faz com que as intensidades espalhadas sejam reduzidas por um fator $\mathrm{p}$ que corrige o efeito do feixe difratado ser polarizado [Stout e Jensen, 1989].

O fator de polarização independe do método experimental, dependendo apenas do ângulo $\theta$ de Bragg, exceto quando o método usa monocromador. Na coleta de dados, utilizando-se o difratômetro CAD-4 da Enraf-Nonius, que possui cristal monocromador, o fator de polarização é dado por [Enraf-Nonius, 1990]:

$$
\mathrm{p}=\left[(\text { Perf }) \frac{\cos ^{2} \theta_{\mathrm{m}}+\cos ^{2} 2 \theta}{1+\cos ^{2} 2 \theta_{\mathrm{m}}}+(1-\text { Perf }) \frac{\cos 2 \theta_{\mathrm{m}}+\cos ^{2} 2 \theta}{1+\cos 2 \theta_{\mathrm{m}}}\right]
$$

onde, Perf é uma constante que depende da mosaicidade do cristal monocromador (para o difratômetro CAD4 assume-se Perf com valor 0,5$) ; \theta_{\mathrm{m}}$ é o ângulo de Bragg do cristal monocromador ( $12.2^{\circ}$ para radiação de $\left.\mathrm{Mo}\right)$; e $\theta$ é o ângulo de Bragg.

\subsection{2 - FATOR DE LORENTZ}

O fator de Lorentz [Stout e Jensen, 1989] surge nos cálculos de redução de dados, devido aos pontos no espaço recíproco atravessarem a esfera de reflexões de Ewald com velocidades diferentes. Este fator depende da técnica experimental utilizada na coleta dos dados de intensidade, e no caso do difratômetro CAD-4, o fator de Lorentz é dado por: 


$$
\mathrm{L}=\frac{1}{\operatorname{sen} 2 \theta} \cdot \frac{1}{\cos \theta}=\frac{1}{\operatorname{sen} 2 \theta}
$$

\subsubsection{3 - FATOR DE ABSORÇÃO}

O fator de absorção está relacionado com o fato que quando os raios-X atravessam um material ocorre uma progressiva diminuição da sua intensidade [Azároff, 1968].

A relação entre os raios- $X$ incidentes e os raios- $X$ refletidos são obtidos a partir da figura 13.

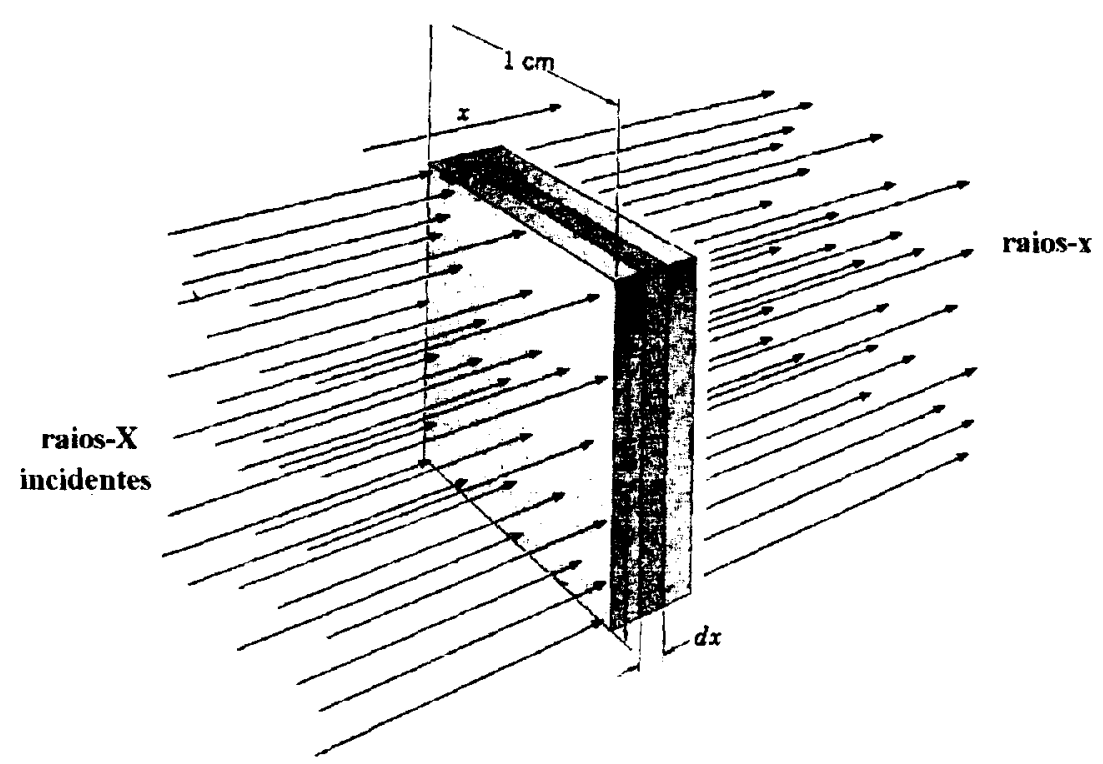

Figura 13 - Relação entre os raios-X incidentes e os raios-X refletidos em um elemento de volume dx.

Observando-se a figura 13 verifica-se que o elemento de volume do material será dado por: $d v=1 \times 1 \times d x\left(\mathrm{~cm}^{3}\right)$. Se a densidade do material é $\rho$, então a fatia $\mathrm{dx}$, 
contém nodv elétrons, e a perda da radiação total espalhada $(\mathrm{dP})$ em todas as direções desta fatia de volume dv é dada por:

$$
\mathrm{dP}=\mathrm{I} \sigma_{\mathrm{e}} \mathrm{n} \rho \mathrm{dv}
$$

O decrescimo nas intensidades dos raios espalhados pela fatia dx é então:

$$
\mathrm{dI}=-\mathrm{I} \sigma_{\mathrm{e}} \mathrm{n} \rho \mathrm{dx}
$$

Integrando a equação 60 ,

$$
\int \mathrm{d} / / \mathrm{I}=-\int \sigma_{\mathrm{e}} \mathrm{n} \rho \mathrm{dx}
$$

obtem-se:

$$
\ln I=-\sigma_{\mathrm{e}} n \rho x+\text { constante }
$$

Considerando a face da superfície, onde $\mathrm{x}=0$, a intensidade dos raios incidentes é $\mathrm{I}_{\mathrm{o}}$, então a expressão 62 torna-se:

$$
\ln \mathrm{I}_{\mathrm{o}}=0+\text { constante }
$$

Substituindo a expressão 63 em 62 obtem-se:

$$
\mathrm{I}=\mathrm{I}_{0} \cdot \mathrm{e}^{-\sigma \rho x}
$$

mas, a capacidade de um material absorver raios-X é expressa pelo coeficiente de absorção de massa $\left(\mu_{\mathrm{m}}\right)$ que é definido como:

$$
\mu_{\mathrm{m}}=\mu / \rho
$$

Portanto a expressão 64 torna-se :

$$
I=I_{0} \cdot e^{-\mu x}
$$

Já no caso de um composto, a absorção dependerá essencialmente dos elementos presentes e das proporções em que se encontram, então o coeficiente de absorção de massa de um composto pode ser calculado pela expressão 67

$$
\mu=d \Sigma P(\mu / \rho)
$$

onde d é a densidade do composto e P é a porcentagem dos elementos presentes. 


\section{8 - FATOR DE TEMPERATURA E SUA RELAÇÃO COM A INTENSIDADE}

Em todo o tratamento de espalhamento feito nas seções anteriores, os átomos foram considerados como pontuais e fixados na rede, sem nenhum movimento vibratório térmico.

$\mathrm{O}$ efeito de vibração térmica também afeta as intensidades dos raios-X difratados porque os átomos, em um cristal, estão sempre vibrando sobre as suas posições e, portanto a magnitude de vibração, depende da temperatura, da massa do átomo, e das forças intermoleculares [Willis e Pryor, 1975]. Desta forma átomos ligados em cadeias, anéis, ou o tipo de átomos que estão ligados, afetam consideravelmente o efeito de vibração.

O efeito de vibração, é o de espalhar a nuvem eletrônica sobre um volume e assim causar uma diminuição do poder de espalhamento do átomo real Então a variação no poder de espalhamento é dada por:

$$
\mathrm{e}^{-\mathrm{B}\left(\frac{\operatorname{sen}^{2} \theta}{\lambda^{2}}\right)}
$$

onde, $\mathrm{B}$ é o fator de temperatura atômico dado por $\mathrm{B}=8 \pi^{2} \mathrm{u}^{2}$, sendo u é o deslocamento atômico médio.

Necessita-se agora encontrar uma expressão para o fator de espalhamento atômico para o átomo real, ou seja , o fator de espalhamento atômico pode ser escrito como [Stout e Jensen, 1989]:

$$
\left.\mathrm{f}=\mathrm{f}_{0} \mathrm{e}^{-\mathrm{B}\left(\operatorname{sen}^{2} \theta\right.} \lambda^{2}\right)
$$

Como a intensidade média observada (ou medida) $\left(\mathrm{I}_{\mathrm{rel}}\right)$ corrigida pelo efeito $\mathrm{Lp}$ é dada por:

$$
\mathrm{I}_{\text {rel }}=\left\langle\left|\mathrm{F}_{\text {rel }}\right|^{2}\right\rangle_{\text {média }}
$$


e considerando uma cela unitária que contém $\mathrm{N}$ átomos, a intensidade média absoluta será dada por:

$$
\mathrm{I}_{\mathrm{abs}}=\sum_{i=1}^{N} \mathrm{f}_{\mathrm{i}}^{2}
$$

ou ainda,

$$
\mathrm{I}_{\mathrm{abs}}=\sum_{i=1}^{N} \mathrm{f}_{\mathrm{oi}}^{2} \mathrm{e}^{-2 \mathrm{~B}\left(\frac{\operatorname{sen}^{2} \theta}{\lambda^{2}}\right)}
$$

Desde que a somatória é feita apenas sobre os fatores de espalhamento, tem-se:

$$
\mathrm{I}_{\mathrm{abs}}=\mathrm{e}^{-2 \mathrm{R}\left(\frac{\operatorname{sen}^{2} \theta}{\lambda^{2}}\right)} \sum_{i=1}^{N} \mathrm{f}_{\mathrm{oi}}^{2}
$$

Como a intensidade média real (ou absoluta) $\left(\mathrm{I}_{\mathrm{abs}}\right)$ é proporcional a intensidade média relativa pode-se escrever:

$$
\mathrm{I}_{\mathrm{rel}}=\mathrm{CI}_{\mathrm{abs}}
$$

ou,

$$
\mathrm{I}_{\mathrm{rel}}=\mathrm{Ce} \mathrm{e}^{-2 \mathrm{~B}\left(\frac{\operatorname{sen}^{2} \theta}{i^{2}}\right)} \sum_{i=1}^{N} \mathrm{f}_{\mathrm{ni}}^{2}
$$

onde $\mathrm{C}$ representa o fator de escala entre a intensidade medida, dependente do método e aparelho de medida, e a intensidade real ( proporcional aos elétrons espalhadores) [Stout e Jensen, 1989].

Reescrevendo-se a equação 75 ,

$$
\frac{\mathrm{I}_{\mathrm{rel}}}{\sum_{i=1}^{N} \mathrm{f}_{\mathrm{oi}}^{2}}=\mathrm{Ce} \mathrm{e}^{-2 \mathrm{R}\left(\frac{\operatorname{sen}^{2} \theta}{i^{2}}\right)}
$$

e finalmente aplicando logaritmo natural obtem-se:

$$
\ln \left(\frac{\mathrm{I}_{\mathrm{rel}}}{\sum_{\mathrm{i}=1}^{\mathrm{N}} \mathrm{f}_{\mathrm{o}}^{2}}\right)=\ln \mathrm{C}-2 \mathrm{~B} \frac{\operatorname{sen}^{2} \theta}{\lambda^{2}}
$$


A equação 77 representa a equação de uma reta, e graficando-se: $\ln \left(\mathrm{I}_{\mathrm{rel}} / \Sigma \mathrm{f}_{\mathrm{oi}}{ }^{2}\right)$ contra $\left(\operatorname{sen}^{2} \theta / \lambda^{2}\right)$, obtêm-se uma reta onde o coeficiente angular é $-2 B$ e o coeficiente linear é ln C. O gráfico assim obtido é conhecido como Gráfico de Wilson e está representado na figura 14 .

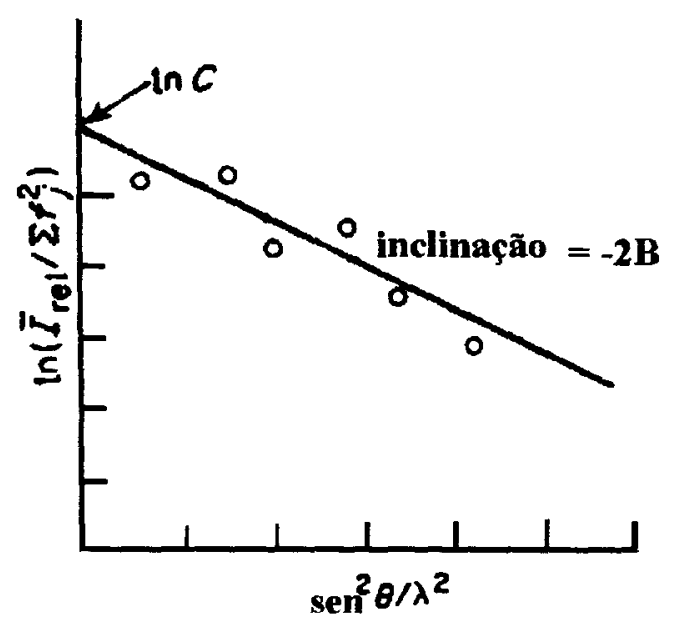

Figura 14 - Gráfico de Wilson para a determinação dos fatores de escala e temperatura isotrópico médio.

\section{9- DENSIDADE ELETRÔNICA E FATOR DE ESTRUTURA}

Como abordado anteriormente, a onda resultante espalhada por uma cela unitária foi definida como o fator de estrutura $\left(\mathrm{F}_{\mathrm{hk} l}\right)$. O fator de estrutura, pode então ser considerado como a soma de pequenas ondas, espalhadas por todos os elementos infinitesimais da densidade eletrônica ( $\rho(x y z))$ de uma cela unitária[Stout e Jensen, 1989].

Como a densidade eletrônica $\rho(x y z)$ é definida como sendo o número de elétrons por unidade de volume, então o número de elétrons em algum elemento de volume $\mathrm{dv}$ será dado por:

$$
\rho(x, y, z) d v=\rho(r) d v
$$

ou na forma exponencial, 


$$
\rho(x, y, z) e^{-2 \pi i(h x+k y+k z)} d v=\rho(r) e^{-2 \pi i(h . r)} d v
$$

Então, a partir das densidades eletrônicas, a soma das pequenas ondas espalhadas por uma cela unitária é dada pela integral sobre o volume:

$$
F_{h k l}=\int \rho(x, y, z) e^{-2 \pi i(h x+k y+h)} d v=\int \rho(r) e^{-2 \pi i(h . r)} d v
$$

Através da expressão 80 , conhecendo-se a densidade eletrônica no espaço recíproco pode-se calcular os $\mathrm{F}_{\mathrm{hk} l}$ no espaço direto, a operação inversa será discutida logo a seguir.

\subsection{1 -SÉRIES DE FOURIER}

Como os cristais são estruturas de natureza periódica, ou seja, os átomos estão arranjados no espaço em uma forma periódica, a densidade eletrônica é também uma função periódica da posição destes átomos, e desta forma, analisa-se a densidade eletrônica por funções periódicas denominadas séries de Fourier [Azároff, 1968].

Como um exemplo de obtenção de uma série de Fourier, pode-se considerar o gráfico da figura 15 .

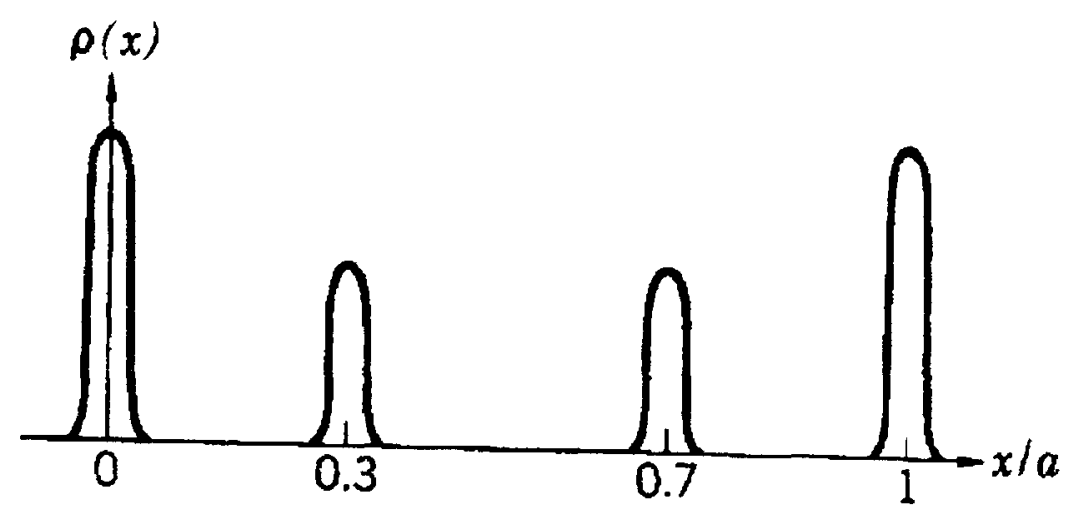

Figura 15. Densidade eletrônica unidimensional simétrica. 
Impondo que cada pico no gráfico da figura 15 , representa a densidade eletrônica $\rho_{\mathrm{a}}$, de um átomo em um cristal unidimensional, então o pico de máxima altura, representa a distribuição da densidade eletrônica $\rho(X)$ no referido cristal, de período translacional a.

Expressando esta densidade eletrônica por uma série de Fourier, em termos de cossenos, pelo fato da simetria ser apropriada tem-se:

$$
\rho(X)=\sum_{n=-\infty}^{+\infty} A_{n} \cos 2 \pi n \frac{X}{a}
$$

onde n é uma variável inteira podendo ser positiva, negativa ou nula; $\mathrm{X}$ é a distância medida ao longo do eixo a.

Os coeficientes podem ser determinados analiticamente, uma vez que a função $\rho(X)$ é conhecida, pelo uso da ortogonalidade da série de Fourier. A multiplicação da equação 81 por $\cos (2 \pi \mathrm{m} \mathrm{X} / \mathrm{a}) \mathrm{dX}$ e posterior integração, sobre o periodo de translação a, resulta em:

$$
\int_{-\frac{a}{2}}^{+\frac{a}{2}} \rho(\mathrm{X}) \cos 2 \pi \mathrm{m} \frac{\mathrm{X}}{\mathrm{a}} \mathrm{dX}=\sum_{n} A_{n} \int_{-\frac{a}{2}}^{+\frac{a}{2}} \cos 2 \pi \mathrm{n} \frac{\mathrm{X}}{\mathrm{a}} \cos 2 \pi \mathrm{m} \frac{\mathrm{X}}{\mathrm{a}} \mathrm{dX}
$$

A integração direta do lado direito da equação torna-se possível após a expansão,

$$
\begin{aligned}
& \int_{-\frac{a}{2}}^{+\frac{a}{2}} \cos 2 \pi n \frac{X}{a} \cos 2 \pi m \frac{X}{a} \mathrm{dX}=\frac{1}{2} \int_{-\frac{a}{2}}^{+\frac{a}{2}} \cos 2 \pi(\mathrm{n}+\mathrm{m}) \frac{\mathrm{X}}{\mathrm{a}} \mathrm{dX}+\frac{1}{2} \int_{-\frac{a}{2}}^{+\frac{a}{2}} \cos 2 \pi(\mathrm{n}-\mathrm{m}) \frac{\mathrm{X}}{\mathrm{a}} \mathrm{dX}= \\
& \quad=\frac{1}{2}\left\{\left[\operatorname{sen} 2 \pi(\mathrm{n}+\mathrm{m}) \frac{\mathrm{X}}{\mathrm{a}}\right]_{-\frac{a}{2}}^{++\frac{a}{2}}+\left[\operatorname{sen} 2 \pi(\mathrm{n}-\mathrm{m}) \frac{\mathrm{X}}{\mathrm{a}}\right]_{-\frac{a}{2}}^{+\frac{a}{2}}\right\}=\frac{1}{2}\left\{\begin{array}{lll}
a & \text { se } \mathrm{n}=\mathrm{m} \\
0 & \text { se } \mathrm{n} \neq \mathrm{m}
\end{array}\right.
\end{aligned}
$$

Através da equação 83 verifica-se que é possível calcular os coeficientes da série de Fourier para cada valor onde $n=m$, através da equação 82 : 


$$
A_{n}=\frac{2}{a} \int_{-\frac{a}{2}}^{+\frac{a}{2}} \rho(x) \cos 2 \pi n \frac{X}{a} d X
$$

Considerando agora a densidade eletrônica tridimensional, de um cristal, podese expressar esta densidade segundo:

$$
\rho(X Y Z)=\sum_{n} \sum_{m} \sum_{p} A_{n m p} e^{-2 \pi i\left(n \frac{X}{a}+m \frac{Y}{b}+p \frac{Z}{c}\right)}
$$

onde as somatórias vão de $-\infty$ a $+\infty$, ou ainda por:

$$
\mathrm{A}_{\mathrm{nmp}}=\mathrm{A}_{\mathrm{hkl}}=\frac{1}{V} \int_{-\frac{a}{2}}^{+\frac{a}{2}} \int_{-\frac{b}{2}}^{+\frac{b}{2}} \int_{-\frac{c}{2}}^{+\frac{c}{2}} \rho(\mathrm{XYZ}) \mathrm{e}^{\left.-2 \pi i \mathrm{~h} \frac{\mathrm{X}}{\mathrm{a}}+\mathrm{k} \frac{\mathrm{Y}}{\mathrm{b}}+l \frac{\mathrm{Z}}{\mathrm{c}}\right)} \frac{V}{a b c} \mathrm{dXdYdZ}
$$

onde $\mathrm{n}=\mathrm{h}, \mathrm{m}=\mathrm{k}, \mathrm{p}=\mathrm{l}$ e V/abc é o fator geométrico de normalização dos coeficientes, para o caso geral de sistemas não ortogonais.

Entretanto, como a densidade eletrônica $\rho(X, Y, Z)$ de um cristal real é considerada como a soma das densidades eletrônicas dos átomos individuais, a equação do fator de estrutura passa a ter a seguinte forma:

$$
\mathrm{F}_{\mathrm{hkl}}=\sum_{\mathrm{n}} \mathrm{f}_{\mathrm{n}} \mathrm{e}^{2 \pi\left(\mathrm{h} \frac{\mathrm{X}}{\mathrm{a}}+k \frac{\mathrm{Y}}{\mathrm{b}}+l \frac{Z}{c}\right)}{ }_{\mathrm{n}}=\iiint_{\text {cela unitaria }} \rho(\mathrm{XYZ}) \mathrm{e}^{\left.-2 \pi i \mathrm{~h} \frac{\mathrm{X}}{\mathrm{a}}+\mathrm{k} \frac{\mathrm{Y}}{\mathrm{b}}+l \frac{Z}{\mathrm{c}}\right)} \frac{\mathrm{V}}{\mathrm{abc}} \mathrm{dX} \mathrm{dY} \mathrm{dZ}
$$

Combinando-se as expressões obtêm-se:

$$
\mathrm{A}_{\mathrm{nmp}}=\mathrm{A}_{\mathrm{hk} l}=\frac{1}{\mathrm{~V}} \mathrm{~F}_{\mathrm{hkl}}
$$

Finalmente pode-se representar a densidade eletrônica sob forma de série de Fourier de acôrdo com a equação:

$$
\rho(\mathrm{xyz})=1 / \mathrm{V} \sum_{h} \sum_{k=-\infty}^{+\infty} \sum_{l} \mathrm{~F}_{\mathrm{hl} l} \mathrm{e}^{-2 \pi\left(h\left(\frac{\mathrm{X}}{\mathrm{a}}+\mathrm{k} \frac{\mathrm{Y}}{\mathrm{b}}+\mathrm{z} \frac{\mathrm{Z}}{\mathrm{c}}\right)\right.}
$$

ou de uma forma mais compacta, como o produto de dois vetores: 


$$
\rho(\mathbf{r})=1 / \mathrm{VF}_{\mathbf{h}} \mathrm{e}^{-2 \pi \mathrm{i}(\mathbf{h} \cdot \mathbf{r})}
$$

A equação 90, apresenta a densidade eletrônica no espaço direto em termos dos fatores de estrutura no espaço recíproco.

Uma outra expressão alternativa para o cálculo da densidade eletrônica, a partir das equações dos fatores de estrutura, pode ser escrita usando-se os módulos de $F_{h k l}$ [Stout e Jensen, 1989]:

$$
F_{h k l}=\left|F_{h k l}\right| \mathrm{e}^{\mathrm{i} \alpha \mathrm{hk} l}=\left|F_{h k l}\right| \mathrm{e}^{2 \pi i \alpha^{\prime} h k l}
$$

onde $\alpha_{\mathrm{hk} /}$ é o ângulo da fase em radianos e $\alpha^{\prime}{ }_{\mathrm{hk} l}$ é o ângulo da fase em ciclos, substituindo a expressão 91 em 90 obtem-se:

$$
\rho(\mathrm{XYZ})=\frac{1}{V} \sum_{h} \sum_{k} \sum_{l}\left|\mathrm{~F}_{\mathrm{hk} l}\right| e^{2 \pi i \alpha_{h k}^{\prime}} \mathrm{e}^{-2 \pi i\left(\mathrm{~h} \frac{\mathrm{X}}{\mathrm{a}}+\mathrm{k} \frac{\mathrm{Y}}{\mathrm{b}}+1 \frac{\mathrm{Z}}{\mathrm{c}}\right)}
$$

ou

$$
\rho(\mathrm{XYZ})=\frac{1}{V} \sum_{h} \sum_{k} \sum_{l}\left|\mathrm{~F}_{\mathrm{hk} l}\right| e^{-2 \pi i\left(\mathrm{~h} \frac{\mathrm{X}}{\mathrm{a}}+\mathrm{k} \frac{\mathrm{Y}}{\mathrm{b}}+1 \frac{\mathrm{Z}}{\mathrm{c}}-\alpha_{h \mathrm{~h} l}^{\prime}\right)}
$$

Expandindo-se esta última expressão em termos de seno e cosseno, usando coordenadas fracionárias xyz, e assumindo a lei de Friedel [Stout e Jensen, 1989] a equação 93 passa a ter a forma:

$$
\rho(\mathrm{xyz})=1 / \mathrm{V} \sum_{h} \sum_{k} \sum_{l}\left|F_{h k l}\right| \cos 2 \pi\left(\mathrm{hx}+\mathrm{ky}+l \mathrm{z}-\alpha_{\mathrm{hk} l}\right)
$$

A equação 94 é a forma tridimensional da série de Fourier aplicada nos cálculos cristalográficos, a partir dos fatores de estrutura observados e das fases calculadas. 


\subsection{0 - O PROBLEMA DA FASE}

Observando-se a expressão 94,verifica-se que se os fatores de estrutura são conhecidos em módulo, a partir do conhecimento das fases, pode-se calcular a densidade eletrônica do cristal, ou da cela unitária, e assim obter a imagem da estrutura do cristal.

Entretanto, no estudo de difração de raios-X por um cristal apenas as intensidades das ondas espalhadas são registradas, e todas as informações a respeito das fases são perdidas.

A razão desta perda é devida ao detector de raios-X, que podendo ser um filme ou um contador, mede somente as intensidades. Como as intensidades são proporcionais ao quadrado do fator de estrutura, que é um número complexo, quando as intensidades são medidas não é possivel separa-las em parte imaginária e real, então as fases são perdidas. Esta perda da informação da fase é, na cristalografia, conhecida como problema da fase [Hukins, 1981],[Bun, 1972].

Tradicionalmente considera-se que existem quatro métodos principais para a solução do problema da fase:

* Métodos Diretos;

* Função de Patterson;

* Substituição Isomorfa;

* Dispersão Anômola.

Métodos Diretos (que serão discutido mais adiante), são métodos puramente matemáticos que permitem determinar as fases de um certo arranjo de $F_{\mathrm{hk} l}$, através de informações físicas e químicas do sistema [Schenk, 1991].

A Função de Patterson consiste em uma série de Fourier que utiliza os valores de $|\mathrm{F}(\mathrm{hk} l)|^{2}$ como coeficientes da série, ao invés de $\mathrm{F}(\mathrm{hk} l)$, o que produz informações a 
respeito da estrutura. Esta função fornece um mapa dos vetores entre os átomos,e estas informações podem então ser aperfeiçoadas por refinamentos [Sands, 1993].

O método de substituição isomorfa é aplicado para determinar estruturas que contém apenas átomos leves, usando-se o artifício de introduzir nesta estrutura um átomo pesado, desde que se obtenham cristais isomorfos, que passa a ser um átomo marcador para a obtenção das fases [Borges, 1980].

Dispersão anômola [Sands, 1993] é o método no qual a informação das fases é obtida pelo fato do comprimento de onda, da radiação incidente, ser próximo à descontinuidade de absorção de um dos átomos da rede cristalina, ocorrendo então uma interação com a frequência de vibração dos elétrons desse átomo, resultando em uma acentuada modificação da amplitude da radiação por ele dispersada. Neste método explora-se a lei de Friedel, uma vez que, neste caso $|\mathrm{F}(\mathbf{h})| \neq|\mathrm{F}(-\mathbf{h})|$.

\subsection{1 - MÉTODOS DIRETOS}

Todas as informações sobre as fases são perdidas no experimento de coleta de dados por difração de raios-X, pois as informações são apenas as intensidades dos raios difratados. Os Métodos Diretos usados para determinar as fases, são métodos puramente matemáticos que para, o estabelecimento de relações matemáticas, exigem que sejam impostas algumas restrições sobre o sistema. Estas restrições são:

a- a não negatividade da densidade eletrônica ou seja $(\rho \geq 0)$;

b- os átomos devem ser considerados como pontuais, e portanto iguais.

Este método é muito utilizado na determinação de fases, principalmente em compostos orgânicos, onde átomos de carbono,oxigênio e nitrogênio, possuem número de elétrons pequeno e bastante próximos. 


\subsection{1 - DESIGUALDADES DE HARKER e KASPER}

Os métodos diretos tiveram as suas origens nas relações de desigualdades, desenvolvidas por Harker e Kasper [1948]. As expressões desenvolvidas, proporcionaram os primeiros meios para a determinação da fase de uma reflexão em termos de sua magnitude.

Como um exemplo [Azároff, 1968] de obtenção da desigualdade de HarkerKasper, parte-se de desigualdade de Schwartz:

$$
\left|\int f g d r\right|^{2} \leq\left(\int|f|^{2} d r\right)\left(\int|g|^{2} d r\right)
$$

impondo os valores adequados na equação,

$$
\begin{aligned}
& f=[V \rho(x y z)]^{1 / 2} \\
& g=[V \rho(x y z)]^{1 / 2} e^{-2 \pi i(h x+k y+l z)} \\
& d \tau=d x d y d z
\end{aligned}
$$

Substituindo agora as equações no lado esquerdo da expressão 95 tem-se:

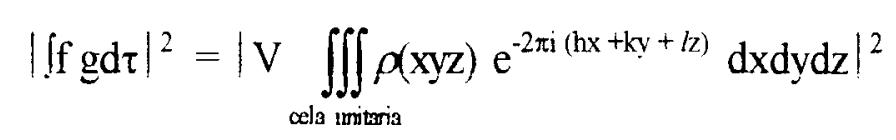

ou,

$$
\left|\int f g d \tau\right|^{2}=\left|F_{h k}\right|^{2}
$$

Substituindo os mesmos valores, agora no direito da equação e lembrando que $\left|\mathrm{e}^{2 \pi \mathrm{i}}\right|^{2}=1$ a desigualdade torna-se:

$$
\left|F_{\text {likl }}\right|^{2} \leq V^{2}\left[\iiint_{\text {celn unteria }} \rho(x y z) d x d y d z\right]^{2}
$$

ou de uma outra forma:

$$
\left|F_{h k}\right|^{2} \leq \xi
$$

sendo $\xi$ o número total de elétrons contidos na cela unitária.

Supondo agora, que o cristal tem centro de simetria, então neste caso tem-se: 


$$
\rho(\mathrm{xyz})=\rho(\overline{\mathrm{x}} \overline{\mathrm{y}} \overline{\mathrm{z}})
$$

e

$$
F_{\mathrm{hk} l}=\mathrm{V} \iiint_{\text {ola }} \rho(\mathrm{xyz}) \cos 2 \pi(\mathrm{hx}+\mathrm{ky}+l \mathrm{z}) \mathrm{dx} \mathrm{dy} \mathrm{dz}
$$

A desigualdade de Schwartz torna-se:

$$
\left|\mathrm{F}_{\mathrm{lk} k l}\right|^{2} \leq\left[\mathrm{V} \iiint_{\text {cela unitrais }} \rho(\mathrm{xyz}) \mathrm{dxdydz}\right]\left[\mathrm{V} \iiint_{\text {cods uniaris }} \rho(\mathrm{xyz}) \cos ^{2} 2 \pi(\mathrm{hx}+\mathrm{ky}+l \mathrm{z}) \mathrm{dxdydz}\right]
$$

Fazendo agora uso da relação do termo trigonométrico $\cos ^{2} x=1 / 2(1+\cos 2 x)$ e observando que o primeiro termo na equação 105 é igual a $\xi$ obtem-se:

$$
\left|F_{h k l}\right|^{2} \leq \xi / 2\left[V \iiint \rho(x y z) d x d y d z+V \iiint \rho(x y z) \cos 2 \pi(2 h x+2 k y+2 l z) d x d y d z\right] \leq \xi / 2\left(\xi+F_{2 h 2 k 2 /}\right)
$$

Tomando a equação anterior e definindo $F_{\mathrm{hk} k} \xi$ como o fator de estrutura unitário $\left(\mathrm{U}_{\mathrm{hk} l}\right)$ (que será discutido na próxima seção) a desigualdade é reescrita finalmente como:

$$
\left|\mathrm{U}_{\mathrm{hk} /}\right|^{2} \leq 1 / 2\left( \pm 1 / 2\left|\mathrm{U}_{2 \mathrm{~h}, 2 \mathrm{k}, 2 l}\right|\right)
$$

Esta desigualdade proposta por Harker e Kasper, possibilita a dedução das fases diretamente das amplitudes dos fatores de estrutura.

Para exemplificar o uso desta relação sobre as fases, pode-se supor que:

$$
\left|U_{h}\right|=0.6 \quad \text { e } \quad U_{2 h}= \pm 0.5
$$

a desigualdade fornece $0.36 \leq(1 / 2)(1 \pm 0.5)$, que somente será satisfeita se o sinal de $U_{2 h}$ for positivo, ou seja implicando que a fase de $U_{2 h}$ é $2 \pi$.

Esta desigualdade inaugurou uma nova era na determinação de estruturas, embora, a sua utilização seja de uso limitado, ou seja, permite resolver apenas aquelas estruturas que contenham um número pequeno de átomos na cela unitária. 


\subsection{2 - FATOR DE ESTRUTURA UNITÁRIO}

Todo o procedimento efetuado no desenvolvimento das desigualdades, até o momento, considerou a expressão para o fator de estrutura, assumindo-se os átomos como sendo pontuais. Considerando-se agora uma cela unitária contendo apenas uma espécie de átomos o fator de estrutura pode ser escrito na forma:

$$
\mathrm{F}_{\mathrm{hk} l}=\mathrm{f} \sum \mathrm{e}^{2 \pi \mathrm{i}(\mathbf{h} \cdot \mathbf{r})}
$$

onde a somatória é feita sobre os $\mathrm{N}$ átomos da mesma espécie;

ou, de outra forma:

$$
\mathrm{F}_{\mathrm{hk} l}=\mathrm{f} . \mathrm{E}
$$

onde E é a soma dos termos exponenciais.

Tomando-se agora a razão entre os fatores de espalhamento para um átomo pontual e o real, e assumindo $f=Z$ ( $Z=$ número atômico $)$ para átomos estacionários pontuais, obtem-se a relação:

$$
\frac{F_{\text {pmitual }}}{F_{\text {real }}}=\frac{Z E}{E f_{0} e^{-B\left(\operatorname{sen}^{2} \theta\right) / \lambda^{2}}}
$$

portanto:

$$
F_{\text {pontual }}=\frac{Z E}{\mathrm{E}_{0} \mathrm{e}^{-\mathrm{B}\left(\operatorname{sen}^{2} \theta\right) / \lambda^{2}}} \mathrm{~F}_{\text {real }}
$$

Mas, como na maioria dos casos os cristais possuem mais do que uma espécie

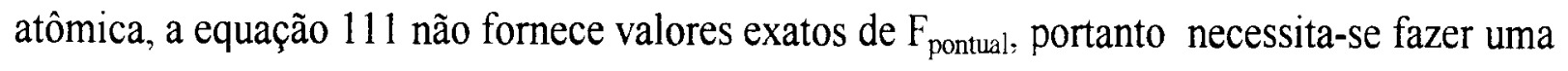
aproximação na relação Z/f, para que seja utilizada nos casos gerais:

$$
\mathrm{F}_{\text {pontual }}=\frac{\sum_{j}^{N} \mathrm{Z},}{\left(\mathrm{e}^{-\mathrm{B}\left(\operatorname{sen}^{2} \theta\right) \cdot \lambda^{2}}\right) \sum_{j}^{N} \mathrm{f}_{0 j}} \mathrm{~F}_{\text {reúl }}
$$


Na prática define-se o fator de estrutura unitário como:

$$
\mathrm{U}_{\mathrm{hk} l}=\frac{\mathrm{F}_{\mathrm{hk} /(\text { pontual })}}{\mathrm{F}_{000}}
$$

Sendo que $\sum_{j}^{N} Z_{j}=\mathrm{F}_{000}$, e utilizando a expressão 113 , tem-se:

$$
\mathrm{U}_{\mathrm{hk} l}=\frac{\mathrm{F}_{\mathrm{hk} l}}{\left(\mathrm{e}^{-\mathrm{B}\left(\operatorname{sen}^{2} \theta\right) / \lambda^{2}}\right) \sum_{j}^{N} \mathrm{f}_{0 j}}
$$

ou de uma forma mais geral, incluindo o fator de temperatura em $\mathrm{f}$ :

$$
\mathrm{U}_{\mathrm{hk} l}=\frac{\mathrm{F}_{\mathrm{hk} l}}{\sum_{j}^{N} \mathrm{f}_{j}}
$$

onde $\mathrm{N}$ é o número total de átomos na cela unitária.

Se a estrutura é centrossimétrica, tem-se:

$$
\mathrm{U}_{\mathrm{hk} l}=2 \sum_{j}^{\frac{N}{2}} \mathrm{n}_{\mathrm{j}} \cos 2 \pi\left(h x_{j}+k y_{j}+l \mathrm{z}_{\mathrm{j}}\right)
$$

onde $n_{j}$ corresponde a $f_{j} / \Sigma f_{j}$. Para o caso de todos os átomos da estrutura iguais $n_{j}$ será dado por $n_{j}=1 / N$.

Quanto aos valores médios dos fatores de estrutura e dos fatores de estrutura unitários as equações podem ser escritos na seguinte forma:

$$
\begin{aligned}
& \left\langle\mathrm{F}^{2}\right\rangle=\sum_{j}^{N} \mathrm{f}_{\mathrm{j}}^{2} \\
& \left\langle\mathrm{U}^{2}\right\rangle=\sum_{j}^{N} \mathrm{n}_{\mathrm{j}}^{2}
\end{aligned}
$$




$$
\langle\mathrm{U}\rangle=\left(\sum_{j}^{N} n_{j}^{2}\right)^{1 / 2}
$$

A equação 119 representa uma boa aproximação quando a estrutura, a ser resolvida, é de um composto orgânico, sem átomos pesados e com todos os átomos com aproximadamente o mesmo número de elétrons.

\subsection{3 - FATOR DE ESTRUTURA NORMALIZADO}

Karle e Hauptman [1956] introduziram pela primeira vez o termo fator de estrutura normalizado $\left(\mathrm{E}_{\mathrm{h} k l}\right)$ dado por:

$$
\mathrm{E}_{\mathrm{hk} l}^{2}=\frac{\mathrm{U}_{\mathrm{hk} l}^{2}}{\left\langle\mathrm{U}^{2}\right\rangle}
$$

nos quais os valores de $\mathrm{E}_{\mathrm{hk} l}$ apresentam certas conveniências matemáticas, permitindo o uso de cálculos probabilísticos além da normalização de todas as classes de reflexões em uma base comum.

Combinando-se as equações 120 e 118 dentro de uma forma geral tem-se:

$$
\mathrm{E}_{\mathrm{hk} J}^{2}=\frac{\mathrm{U}_{\mathrm{hk} l}^{2}}{\varepsilon \sum_{j}^{N} \mathrm{n}_{\mathrm{j}}^{2}}
$$

ou, de forma equivalente, reescrevendo a equação anterior usando módulos: 


$$
\left|\mathrm{E}_{\mathrm{hk} l}\right|^{2}=\frac{\left|\mathrm{F}_{\mathrm{hkl}}\right|^{2}}{\varepsilon \sum_{j}^{\mathrm{N}} \mathrm{f}_{\mathrm{j}}^{2}}
$$

Da equação 122, pode-se observar que: $\left|E_{\text {hkl }}\right|^{2}$ é o fator de estrutura normalizado; $\mathrm{f}_{\mathrm{j}}$ é o fator de espalhamento atômico e $\varepsilon$ é um número inteiro que leva em conta a simetria do grupo espacial, garantindo que todos os tipos de reflexões sejam consideradas ou seja:

$$
\left|\mathrm{E}_{\mathrm{hk} l}\right|^{2} \geq 1
$$

Os fatores de estruturas normalizados, $\left|\mathrm{E}_{\mathrm{hk} l}\right|$, são calculados na suposição que os átomos são pontuais, e a distribuição dos valores de $\left|E_{\mathrm{hk}}\right|$ é independente do conteúdo e tamanho da cela unitária, e quando não dispomos da geometria do conteúdo da cela unitária supõe-se que os átomos estejam aleatoriamente distribuídos na cela. Então de acordo com a equação 122 os fatores de estrutura normalizados dependem da presença ou da ausência de um centro de simetria do grupo espacial. A tabela 1 fornece os valores esperados para os casos centrossimétrico e assimétrico [Stout e Jensen, 1989].

\section{Tabela 1}

\begin{tabular}{|c|c|c|}
\hline \multicolumn{2}{|c|}{ Valores teóricos relacionados aos $|\mathbf{E}|$} \\
\hline Valores médios & Centrossimétrico & Assimétrico \\
\hline$|E|^{2}$ & 1,0000 & 1,0000 \\
\hline$\left|E^{2}-1\right|$ & 0,968 & 0,736 \\
\hline$|E|$ & 0,798 & 0.886 \\
\hline$|E|>1$ & $32,0 \%$ & $36,8 \%$ \\
\hline$|E|>2$ & $5,0 \%$ & $1,8 \%$ \\
\hline$|E|>3$ & $0,3 \%$ & $0,01 \%$ \\
\hline
\end{tabular}




\subsection{4 - DETERMINANTE DE KARLE- HAUPTMAN}

Como foi discutido anteriormente a etapa inicial da aplicação dos métodos diretos para a obtenção das fases utiliza as desigualdades de Harker e Kasper.

Utilizando as relações de desigualdades de Cauchy e Schwartz [Azároff, 1968], Karle e Hauptman [1950] derivaram relações entre os fatores de estrutura que resultam na obtenção de suas fases, impondo que a densidade eletrônica $\rho(\mathbf{r})$ seja positiva em todo o espaço.

De acordo com a expressão 89 , que fornece a densidade eletrônica no espaço real, é possivel obte-la também no espaço recíproco. Esta transformação do espaço real para o espaço recíproco foi resolvida por Karle e Hauptman [1950]. definindo a forma hermitiana.

Partindo-se da transformada de Fourier:

$$
\mathrm{F}_{\mathrm{hk}]}=\mathrm{V} \iiint \rho(\mathrm{x}, \mathrm{y}, \mathrm{z}) \exp ^{-2 \pi \mathrm{i}[(\mathrm{hx}+\mathrm{ky}+l z)]} \mathrm{dx} \mathrm{dy} \mathrm{dz}
$$

a forma hermitiana pode ser escrita:

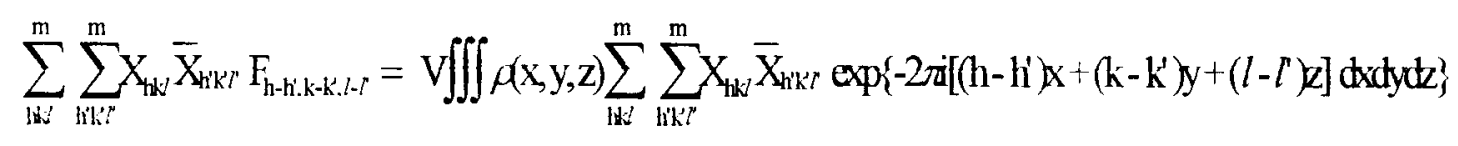

onde: $\mathrm{m}=1,2, \ldots, \mathrm{n} ; \mathrm{X}_{\mathrm{hk} /}$ é uma variável independente; $\overline{\mathrm{X}}_{\mathrm{h} \mathrm{k}^{\prime} \mathrm{l}}$ é o complexo conjugado de $X_{h k l}$; a integral tripla é feita entre 0 e 1,e a somatória representa uma somatória tripla.

Expressando-se o lado direito da equação como o produto de uma soma tripla e seu complexo conjugado, então a expressão 125 torna-se: 


$$
\begin{aligned}
& \mathrm{V} \iiint \rho(\mathrm{x}, \mathrm{y}, \mathrm{z}) \sum_{\mathrm{hk} l}^{\mathrm{m}} \sum_{\mathrm{h}^{\prime} \mathrm{k} l^{\prime}}^{\mathrm{m}} \mathrm{x}_{\mathrm{hk} l^{\prime}} \overline{\mathrm{X}} \mathrm{h}^{\prime} \mathrm{k}^{\prime} l^{\prime} \exp \left\{-2 \pi\left[\left(\mathrm{h}-\mathrm{h}^{\prime}\right) \mathrm{x}+\left(\mathrm{k}-\mathrm{k}^{\prime}\right) \mathrm{y}+\left(l-l^{\prime}\right) \mathrm{zdxdydz}\right]=\right. \\
& =\mathrm{V} \iint \rho \rho(\mathrm{x}, \mathrm{y}, \mathrm{z}) \sum_{\mathrm{hk} l}^{\mathrm{m}} \mathrm{X}_{\mathrm{hk} l} \exp \{-2 \pi \mathrm{i}(\mathrm{hx}+\mathrm{hy}+l \mathrm{z})\}^{2} \mathrm{dxdydz}
\end{aligned}
$$

Como $\rho(x, y, z)$ é uma função não negativa, o lado esquerdo da equação 126 consiste de formas hermitianas não negativas escritas como:

$$
\sum_{\mathrm{h} k l}^{\mathrm{m}} \sum_{\mathrm{h}^{\prime} \mathrm{k}^{\prime} l^{\prime}}^{\mathrm{m}} \mathrm{X}_{\mathrm{hk} l} \overline{\mathrm{X}}_{\mathrm{h}^{\prime} \mathrm{k}^{\prime} l^{\prime} l^{\prime}} \mathrm{F}_{\mathrm{h}-\mathrm{h}^{\prime}, \mathrm{k}-\mathrm{k}^{\prime}, l-l^{\prime}} \geq 0 \quad \mathrm{~m}=1,2,3 \ldots \ldots
$$

Como a forma hermitiana requer a condição de não negatividade, um sistema de determinantes envolvendo fatores de estrutura também deverá ser Não negativo [Karle, 1976], então a partir desta condição, pode-se mostrar que esta condição implica em:

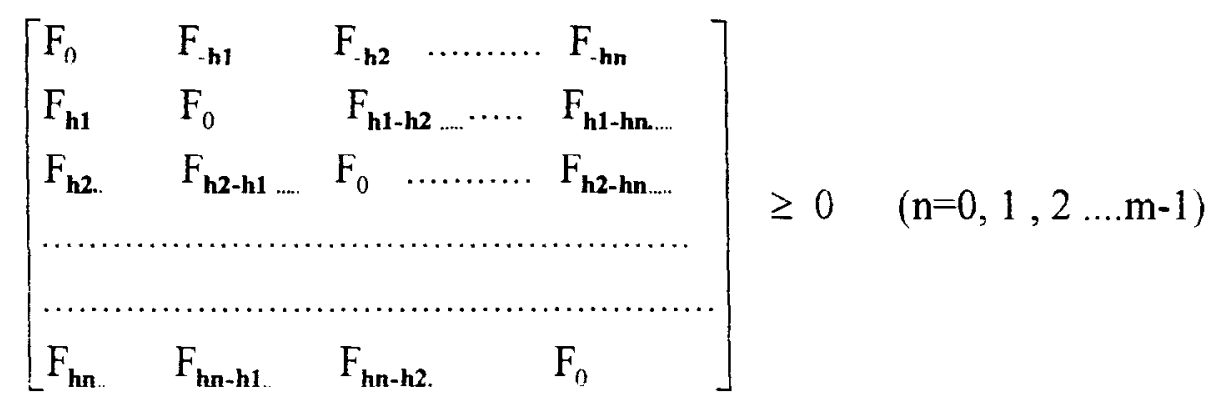

O determinante expresso em 128 é conhecido por determinante de KarleHauptman, podendo ser de qualquer ordem, e devido ao fato da densidade eletrônica ser sempre positiva, o determinante também o será, verificando-se que, quanto ao modo de sua obtenção, não é feita nenhuma suposição prévia a respeito das formas da densidade eletrônica.

Uma maneira mais geral é utilizar o determinante na forma de fatores de estrutura unitários, o que ao mesmo tempo remove a dependência dos fatores de estrutura 
com os valores de $\operatorname{sen} \theta / \lambda$. Usando os fatores unitários o novo determinante será de ordem $\mathbf{m}$, e sabendo-se que $U(0)=1$, terá a seguinte forma:

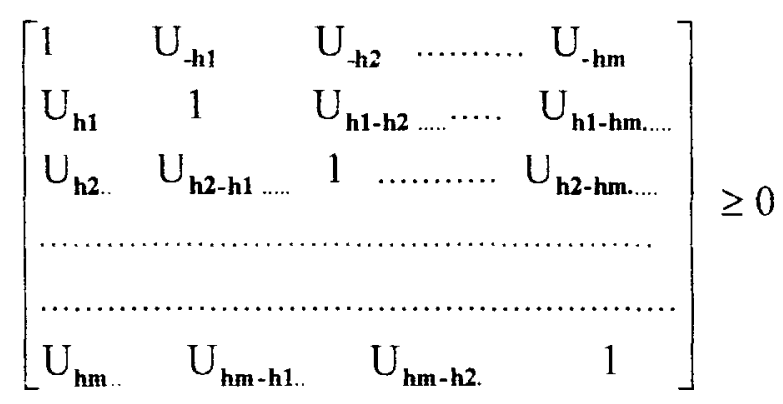

Usando os fatores de estrutura unitários para uma estrutura cristalina centrossimétrica onde $U_{h}=U_{-h}$, e considerando um determinante de ordem três obtêm-se um resultado já familiar:

$$
\left[\begin{array}{ccc}
1 & U_{-h} & U_{-2 h} \\
U_{h 1} & 1 & U_{h 1-h 2} \\
U_{h 2} & U_{h 2-h 1} \ldots . . . & 1
\end{array}\right] \geq 0
$$

Resolvendo o determinante expresso em 130 obtêm-se:

$$
\begin{aligned}
& 1-2\left|U_{h}\right|^{2}-\left|U_{2 h}\right|^{2}+2\left|U_{h}\right|^{2} U_{2 h} \geq 0 \\
& \left(1-U_{2 h}\right)-\left(1+U_{2 h}\right)-2\left|U_{h}\right|^{2}\left(1-U_{2 h}\right) \geq 0 \\
& \left(1-U_{2 h}\right)\left[1+U_{2 h}-2\left|U_{h}\right|^{2}\right] \geq 0
\end{aligned}
$$

e rearranjando, obtêm-se finalmente a desigualdade de Harker e Casper:

$$
\left|U_{h}\right|^{2} \leq 1 / 2\left[1+U_{2 h}\right]
$$

Karle e Hauptman [1950] rearranjaram o determinante 129 para expressar a região de fases para um único fator de estrutura, a partir de dois outros fatores de estrutura, 
cujas fases são conhecidas. Desta forma, assumindo que $U_{h}$ é desconhecido e o restante conhecido, pode-se escrever:

$$
\left|U_{h}-\delta_{h-k}\right| \leq r
$$

onde

$\delta_{h-k}=U_{k} U_{h-k}, e, r=\left[\begin{array}{cc}1 & U_{k}^{*} \\ U_{k} & 1\end{array}\right]^{\frac{1}{2}}\left[\begin{array}{ll}1 & U_{-h+k}^{*} \\ U_{h-k} & 1\end{array}\right]^{\frac{1}{2}}$

A interpretação da equação 132 , usando $F_{h}$ no lugar de $U_{h}$ pode ser representada no diagrama de Argand na figura 16.

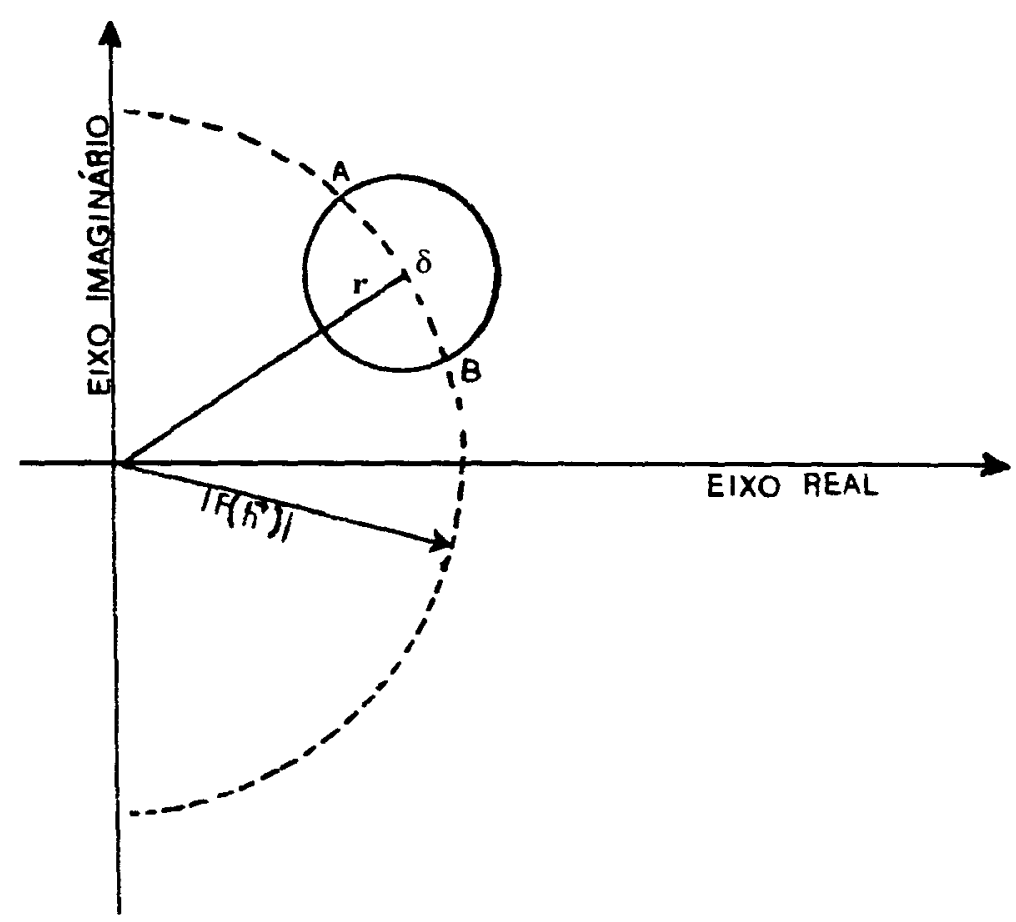

Figura 16 - Ilustração do diagrama de Argand

De acordo com o diagrama da figura 16, o fator de estrutura, $\mathrm{F}_{\mathrm{h}}$, cujo módulo é conhecido, está restrito a um círculo no plano complexo, centrado em $\delta$ e com raio igual a r. 
Este arco (A-B) será tanto menor quanto maiores forem os valores de $\left|F_{\mathbf{h}}\right|$, $\left|F_{\mathbf{k}}\right|$ e $\left|F_{\mathbf{h}-\mathbf{k}}\right|$, ou seja os determinantes tendendo a uma alta ordem, o valor de r pode tornarse muito pequeno ou ser igual a zero.

\subsection{5 - INVARIANTES ESTRUTURAIS [HAUPTMANN E KARLE, 1953]}

Uma das dificuldades na solução do problema da fase é a escolha da origem da cela unitária, com respeito aos elementos de simetria.

Escolhendo-se arbitrariamente a origem da cela, os fatores de estrutura não serão afetados, mas as fases serão drasticamente afetadas. Entretanto, existem certas combinações de fases, cujos valores são determinados pela estrutura isolada e são independentes da escolha da origem, estas combinações lineares de fases são denominadas invariantes estruturais.

Utilizando-se a figura 17, obtêm-se uma expressão quantitativa que fornece a variação da fase, sobre os fatores de estrutura, $\phi_{\mathbf{h}}$, ao variar-se a origem do ponto $O$ para um outro ponto O' sobre um vetor $\mathbf{t}$.

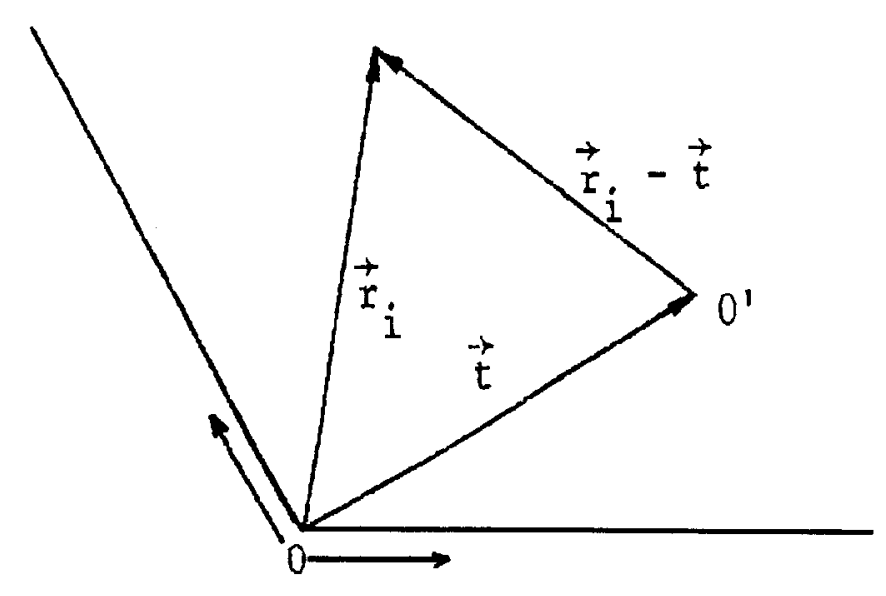

Figura 17 - Deslocamento da origem de 0 para O' 
Definindo $\mathrm{F}_{\mathbf{h}}$ em relação a origem $\mathrm{O}$, e $\mathrm{F}_{\mathbf{h}}$ ' em relação a nova origem $\mathrm{O}^{\prime}$ podese escrever as expressões para os fatores de estrutura na seguinte forma:

$$
\begin{aligned}
& F_{h}=\Sigma f_{j} \exp \left\{2 \pi h \cdot r_{i}\right\} \\
& \mathrm{F}_{\mathbf{h}}{ }^{\prime}=\Sigma \mathrm{f}_{\mathrm{j}} \exp \left\{2 \pi \mathrm{ih} .\left(\mathbf{r}_{\mathbf{i}}-\mathbf{t}\right)\right\} \quad \text { ou } \\
& F_{h}{ }^{\prime}=\Sigma f_{j} \exp \left\{2 \pi i h . r_{j}\right\} \exp \{-2 \pi i h . t\}
\end{aligned}
$$

sendo a somatória sobre os $\mathrm{N}$ átomos; então

$$
F_{h}{ }^{\prime}=F_{h} \exp (-2 \pi i \text { h.t })
$$

ou,

$$
F_{h}^{\prime}=\left|F_{h}\right| \exp \left\{-2 \pi i \mathbf{h . t}+i \phi_{h}\right\}
$$

como $\left|F_{h}\right|=\left|F_{h}\right|$, então:

$$
\phi_{\mathbf{h}}{ }^{\prime}=-2 \pi \mathbf{h . t}+\phi_{\mathbf{h}}
$$

Conclui-se então que as fases dependem da escolha da origem, sendo modificadas por uma quantidade igual a $-2 \pi$ h.t, enquanto, por outro lado, as amplitudes são independentes da escolha da origem.

Desta forma considerando o produto de fatores de estrutura:

$$
F_{h 1} \cdot F_{h 2} \cdot F_{h 3} \cdot
$$

e consequentemente,

$$
\phi_{1}+\phi_{2}+\phi_{3}+
$$


quando: $\mathbf{h}_{1}+\mathbf{h}_{2}+\mathbf{h}_{3}+$ $\equiv 0$, tem se a chamada invariante de estrutura, que é uma quantidade independente da escolha da origem.

Como na difração de raios-X obtem-se informações apenas das amplitudes, o que se quer dizer é que, na prática, qualquer produto de fatores de estrutura, em que a soma dos seus índices for igual a zero deve ser uma invariante estrutural.

Alguns exemplos importantes de invariante de estrutura são:

* $\mathrm{F}(\mathbf{0})$ que depende apenas do conteúdo da cela unitária;

* $F_{h}$. $F_{-h}=\left|F_{h}\right|^{2}$ que estabelece que as amplitudes dos fatores de estrutura são independentes da origem;

$* \mathrm{~F}_{\mathrm{h}} \cdot \mathrm{F}_{\mathrm{k}} \cdot \mathrm{F}_{-\mathrm{h}-\mathrm{k}}$ que é chamado de triplete, relação $\Sigma_{2}$, ou invariante de três fases;

* $\mathrm{F}_{-\mathbf{h}} \mathrm{F}_{-\mathbf{k}} \mathrm{F}_{-l} \mathrm{~F}_{\mathbf{h}+\mathbf{k}+l}$ que são chamados de quartetos invariantes ou invariante de quatro fases.

\subsection{6 - SEMI-INVARIANTES ESTRUTURAIS E DEFINIÇ̃̃O DA ORIGEM}

Semi-invariantes estruturais [Main,1976] são produtos de fatores de estrutura, ou combinações lineares de fases, cujos valores são unicamente determinados pela simetria do cristal e são independentes da escolha da origem desde que a nova origem seja colocada sobre ponto de simetria equivalente.

Para definir a estrutura de um cristal por completo, necessita-se especificar as posições atômicas dentro de uma unidade translacional que é a cela unitária do retículo. De 
fato, a origem da cela unitária pode ser movida livremente dentro de um cristal, mas para especificar as coordenadas atômicas, a origem de uma cela deve ser definida.

A escolha da origem da cela unitária não pode ser feita de uma forma aleatória. Existem posições preferênciais, dependendo então da simetria do cristal. Desta forma para cada grupo espacial existem conjuntos de pontos cuja vizinhança em termos de elementos de simetria é idêntica, e cada um destes conjuntos é chamado de classe de origem equivalente.

Assim por exemplo tomando-se o grupo espacial P1, e definindo a cela unitária no espaço recíproco, representada na figura 18 , onde $\mathrm{V}^{*}$ é o volume do espaço recíproco, e os eixos são definidos pelos vetores $\mathbf{h}_{\mathbf{1}}, \mathbf{h}_{\mathbf{2}} \mathbf{e} \mathbf{h}_{\mathbf{3}}$, não coplanares, pode-se calcular $\mathrm{V}^{*}$ como:

$$
\tau=\mathbf{h}_{1} \bullet \mathbf{h}_{2} \times \mathbf{h}_{3}
$$

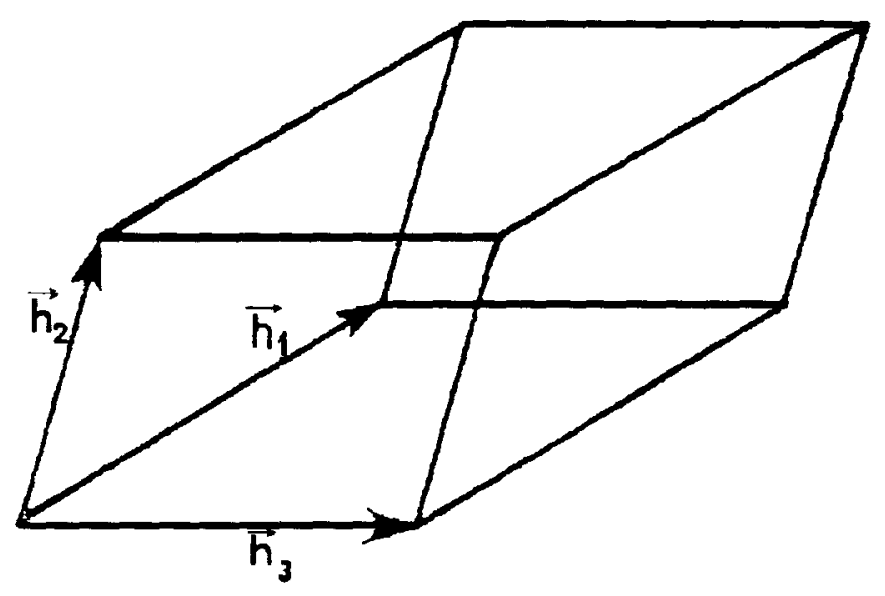

Figura 18- Cela unitária no espaço recíproco.

Escrevendo os vetores em termos de seus componentes tem-se:

$$
\begin{aligned}
& \mathbf{h}_{1}=h_{1} \mathbf{a}^{*}+k_{1} \mathbf{b}^{*}+\mathrm{l}_{1} \mathbf{c}^{*} \\
& \mathbf{h}_{2}=\mathrm{h}_{2} \mathbf{a}^{*}+\mathrm{k}_{2} \mathbf{b}^{*}+\mathrm{l}_{2} \mathbf{c}^{*}
\end{aligned}
$$




$$
\mathbf{h}_{3}=\mathrm{h}_{3} \mathbf{a}^{*+\mathrm{k}_{3}} \mathbf{b}^{*}+\mathrm{l}_{3} \mathbf{c}^{*}
$$

A equação para o volume do paralelepípedo a partir das equações anteriores

torna-se:

$$
\tau=\left(h_{1} \cdot a^{*}+k_{1} \cdot b^{*}+l_{1} \mathbf{c}^{*}\right) \cdot\left(h_{2} a^{*}+k_{2} \mathbf{b}^{*}+l_{2} \mathbf{c}^{*}\right) \cdot\left(h_{3} a^{*}+k_{3} b^{*+1} l_{3} \mathbf{c}^{*}\right)
$$

ou,

$$
\tau=\left(\mathbf{a}^{*} \bullet \mathbf{b}^{*} \times \mathbf{c}^{*}\right)\left\{\mathrm{h}_{1}\left(\mathrm{k}_{2} \mathrm{l}_{3}-\mathrm{k}_{3} \mathrm{l}_{2}\right)+\mathrm{k}_{1}\left(\mathrm{l}_{2} \mathrm{~h}_{3}-\mathrm{l}_{3} \mathrm{~h}_{2}\right)+\mathrm{l}_{1}\left(\mathrm{~h}_{2} \mathrm{k}_{3}-\mathrm{h}_{3} \mathrm{k}_{2}\right)\right.
$$

Expressando a equação anterior em forma de determimante pode-se escrever:

$$
\tau=\left(\mathbf{a}^{*} \bullet \mathbf{b}^{*} \times \mathbf{c}^{*}\right)\left|\begin{array}{lll}
h_{1} & \mathrm{k}_{1} & \mathrm{l}_{1} \\
h_{2} & \mathrm{k}_{2} & \mathrm{l}_{2} \\
h_{3} & \mathrm{k}_{3} & \mathrm{l}_{3}
\end{array}\right|
$$

Como a definição da origem requer $\tau=\mathrm{V}^{*}=\left(\mathbf{a}^{*} \bullet \mathbf{b}^{*} \times \mathbf{c}^{*}\right)$; então $\mathbf{0}$ determinante anterior torna-se:

$$
\left|\begin{array}{lll}
h_{1} & k_{1} & l_{1} \\
h_{2} & k_{2} & l_{2} \\
h_{3} & k_{3} & l_{3}
\end{array}\right|= \pm 1
$$

onde - 1 indica que o conjunto dos 3 vetores $\mathbf{h}_{\mathbf{1}}, \mathbf{h}_{\mathbf{2}}$ e $\mathbf{h}_{\mathbf{3}}$ forma um conjunto "mão esquerda".

Pelas equações 136 e 137, verificou-se que a mudança da origem por um vetor $\mathbf{t}$ deixa inalterado o módulo, mas altera a fase do fator de estrutura.

Considerando o grupo espacial P $\overline{1}$, cuja cela unitária está mostrada na figura 19, pode-se verificar que existem 8 centros de inversão equivalentes. 


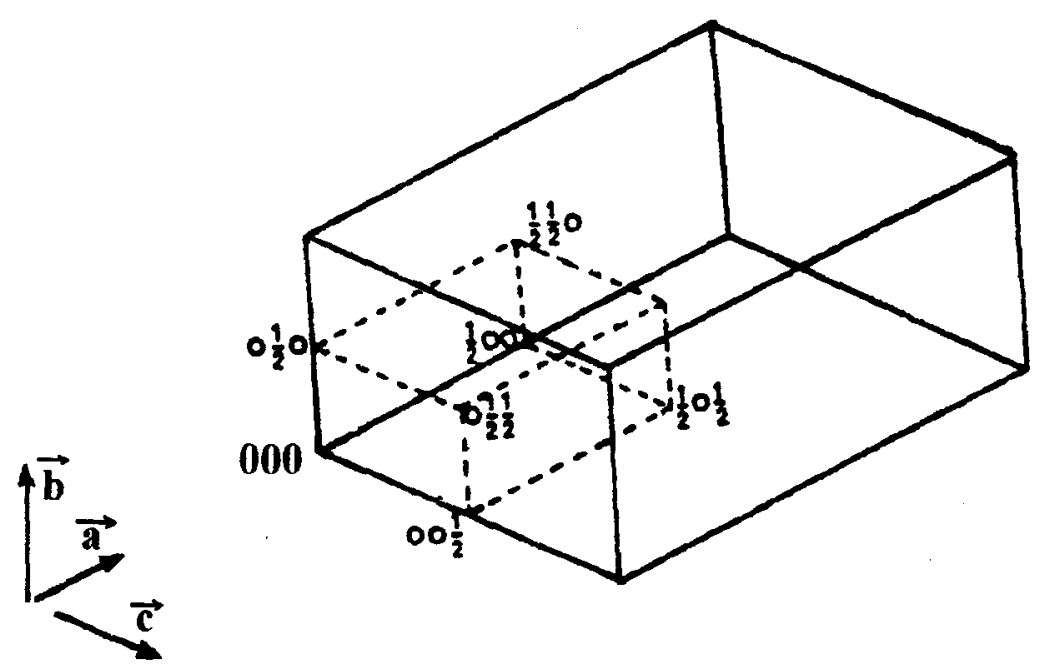

Figura 19 - Origens equivalentes no grupo espacial $\mathbf{P} \overline{1}$.

A equação 137, pode ser reescrita como:

$$
\phi_{\mathbf{h}}^{\prime}=\phi_{\mathbf{h}}-2 \pi \mathbf{h} \Delta \mathbf{x}
$$

onde $\Delta \mathbf{x}$ representa a translação de origem, e então pode-se definir:

$$
\Delta \phi=-2 \pi \mathbf{h} \Delta \mathbf{x}
$$

Para que as fases não sejam alteradas pela mudança de origem é necessário que $\Delta \phi=2 \mathrm{n} \pi$, ou de forma explícita:

$$
\begin{aligned}
& 2 \pi(\mathrm{hx}+\mathrm{ky}+l \mathrm{z})=2 \mathrm{n} \pi \\
& (\mathrm{hx}+\mathrm{ky}+l \mathrm{z})=\mathrm{n}
\end{aligned}
$$

onde $\mathrm{x}, \mathrm{y}, \mathrm{Z}$ são as coordenadas das posições de origem equivalentes e $\mathrm{n}$ é um número inteiro.

Aplicando o mesmo raciocínio para o produto de fatores de estrutura, ou seja para a soma das fases (equação 139), tem-se: 
$\Sigma \mathrm{h}_{\mathrm{i}} \mathrm{x}+\Sigma \mathrm{k}_{\mathrm{i}} \mathrm{y}+\Sigma l_{\mathrm{i}} \mathrm{z}=\mathrm{n}$

Pela figura 19 (grupo espacial $\mathrm{P} \overline{1}$ ) verifica-se que as coordenadas dos centros de inversão possuem valores iguais a 0 e $1 / 2$, para os 3 eixos a, b e c.

Para que a equação 143 seja satisfeita é preciso que $\mathrm{h}_{\mathrm{i}}, \mathrm{k}_{\mathrm{i}}$ e $l_{\mathrm{i}}$ sejam todos pares, o que matematicamente é expresso por: $p(\bmod q)$, ou seja,

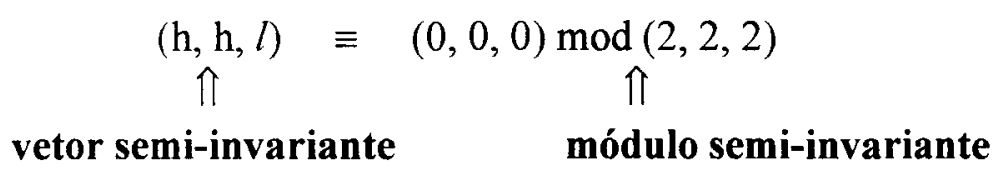

$p(\bmod q)$ significa que $q$ é subtraido de $p$, até que um número $r(0 \leq r \leq q)$ seja

atingido.

Então pode-se concluir que para o grupo espacial $\mathrm{P} \overline{\mathrm{l}}$ as semi-invariantes de estrutura serão as reflexões que tem $\mathrm{hk} l$ todos pares.

Com base nestas considerações pode-se concluir que: " um conjunto de reflexões define a origem se e somente se elas são linearmente independentes e primitivas em relação ao módulo semi-invariante".

Como regra prática para definir a origem em qualquer grupo espacial Hauptmam e Karle [1956] estabeleceram um procedimento simples que consiste em

1 - escolher as p reflexões e reduzi-las ao módulo semi-invariante; o número $p$ é igual ao número de elementos do vetor semi-invariante ( $(\mathrm{hkl}) \bmod (2,2,2)$ implica em 3 reflexões);

2 - montar uma matriz de ordem $p \times p$ das $p$ reflexões escolhidas e calcular o seu determinante;

3 - se o determinante for igual a \pm 1 a origem está fixada, caso contrário não. 
No caso de grupos espaciais não centrossimétricos, além da origem é necessário fixar o enantiomorfo, e isto é feito pela escolha de uma reflexão adicional que seja linearmente dependente daquelas escolhidas para fixar a origem. Esta necessidade surge como consequência direta da definição de estruturas cristalinas enantiomorfas, que são aquelas que embora tenham a mesma origem são relacionadas por diferença de fase, de $\pi$ radianos, em uma reflexão.

\subsection{7 - RELAÇÕES DE PROBABILIDADE}

Para estruturas reais a dimensão dos dados envolvidos, número de reflexões, faz com que somente as desigualdades sejam insuficientes para encontrar uma solução das fases. É então necessário encontrar uma outra forma de abordagem que resulte numa melhor aproximação [Stout e Jensen, 1989].

Pode-se facilmente verificar que há um intervalo de intensidades de reflexões que são pequenas para serem usadas nas desigualdades, mas são suficientemente, ou relativamente, grandes, ou seja estão entre as observáveis. Para estas reflexões é possível estabelecer equações que são provavelmente verdadeiras, e a partir delas extrair informação sobre as suas fases.

Estes métodos, probabilisticos, foram inicialmente introduzidos para o uso em estruturas centrossimetricas e posteriormente estendidos aos grupos não centrossimétricos. 


\subsubsection{1 - MÉTODOS CENTROSSIMÉTRICOS}

As primeiras aproximações para os métodos descritos por Sayre [1952], apresentam resultados equivalentes aos publicados anteriomente por Karle e Hauptman [1950].

Pode ser estabelecido sob certas restrições que:

$$
\mathrm{F}_{\mathrm{hk} l}=\sum_{\mathrm{h}^{\prime}} \sum_{\mathrm{k}^{\prime}} \sum_{l^{\prime}} \mathrm{F}_{\mathrm{h}^{\prime} \mathrm{k},}, \mathrm{F}_{\mathrm{h}-\mathrm{h}^{\prime}, \mathrm{k}-\mathrm{k}^{\prime}, l-r^{\prime}}
$$

A implicação da equação 153 é que qualquer fator de estrutura $F_{h k l}$ pode ser determinado pelo produto de todos os pares de fatores de estrutura, cujos índices adicionados fornecem $\left(\mathrm{hk} l\right.$ ). Assim por exemplo $\mathrm{F}_{213}$ dependerá do produto de $\mathrm{F}_{322}$ e $\mathrm{F}_{\overline{i 1} \mathrm{i}}$.

Segundo a expressão 153, para se determinar um fator de estrutura qualquer, é necessário conhecer as magnitudes e fases de todos os outros, e de acordo com Sayre, para os casos onde $\mathrm{F}_{\mathrm{hk}}$, são altos, as séries devem tender fortemente em uma direção (+ ou -), direção esta que é geralmente determinada pelas concordâncias entre os sinais dos produtos entre os valores de $\mathrm{F}$ grandes. Então para o caso de três reflexões grandes pode-se escrever as seguintes expressões:

$$
\mathrm{s}\left(\mathrm{F}_{\mathrm{hk} l}\right) \approx \mathrm{s}\left(\mathrm{F}_{\mathrm{h}^{\prime} \mathrm{k}^{\prime} l^{\prime}}\right) \cdot \mathrm{s}\left(\mathrm{F}_{\mathrm{h}-\mathrm{h}^{\prime} \cdot \mathrm{k}^{\prime} \cdot \mathrm{k}^{\prime} \cdot l-l^{\prime}}\right)
$$

ou,

$$
\mathrm{s}\left(\mathrm{F}_{\mathrm{hk} l}\right) \cdot \mathrm{s}\left(\mathrm{F}_{\mathrm{h} \cdot \mathrm{k}^{\prime} l}\right) \cdot \mathrm{s}\left(\mathrm{F}_{\mathrm{h}-\mathrm{h}^{\prime} \cdot \mathrm{k}-\mathrm{k}^{\prime} \cdot \mathrm{ll} \mathrm{l}^{\prime}}\right) \approx+1
$$

onde s significa sinal de e $\approx$ indica "provavelmente igual a' e s( ) pode ser considerado como \pm 1 , e geralmente é indicado como s(hkl). 
As equações 154 e 155 são equações de probabilidade derivadas da equação 139 que permite a escolha do sinal da invariante de estrutura.

Cochran e Woolfson [1955] desenvolveram uma expressão para encontrar a probabilidade $(\mathrm{P})$ de um sinal, ou fase:

$$
\mathrm{P}=1 / 2+1 / 2\left\{\operatorname{tgh}\left[\left(\sigma_{3} / \sigma_{2}{ }^{3}\right)\left|\mathrm{U}_{\mathrm{hk} /} \mathrm{U}_{\mathrm{h}^{\prime} \mathrm{k} \%}, \mathrm{U}_{\mathrm{h}-\mathrm{h}^{\prime}, \mathrm{k}-\mathrm{k}^{\prime}, \mathrm{l}-\mathrm{F}}\right|\right]\right\}
$$

onde:

$$
\begin{gathered}
\sigma_{3}=\sum_{j}^{N} \mathrm{n}_{j}{ }^{3} \\
\sigma_{2}=\sum_{j}^{N} \mathrm{n}_{\mathrm{j}}{ }^{2}
\end{gathered}
$$

$\operatorname{com} n_{j}$ definido por $f_{j} / \Sigma f_{j}$.

Para os casos em que os átomos da cela são iguais pode-se facilmente chegar a:

$$
\frac{\sigma_{3}}{\sigma_{2}^{3}}=\frac{\mathrm{N}}{\mathrm{N}^{3}}\left(\frac{\mathrm{N}}{\mathrm{N}^{2}}\right)^{-3}=\mathrm{N}
$$

então:

$$
\mathrm{P}=1 / 2+1 / 2\left\{\operatorname{tgh}\left[\mathrm{N}\left|\mathrm{U}_{\mathrm{hk} l} \quad \mathrm{U}_{\mathrm{h} \cdot \mathrm{k}, \eta}, \mathrm{U}_{\mathrm{h}-\mathrm{h}^{\prime}, \mathrm{k}-\mathrm{k}^{\prime}, \mathrm{l}, \mathrm{H}}\right|\right]\right\}
$$

Reescrevendo a equação 160 em termos de fator de estrutura normalizado $\left(E_{\mathrm{hkl}}\right)$, obtêm-se:

$$
\mathrm{P}=1 / 2+1 / 2\left\{\operatorname{tgh}\left[\left(\sigma_{3} / \sigma_{2}^{3 / 2}\right)\left|\mathrm{E}_{\mathrm{hl} l !} \mathrm{E}_{\mathrm{h}, \mathrm{k},} \mathrm{E}_{\mathrm{h}-\mathrm{h}, \mathrm{k}-\mathrm{k}, \mathrm{l}}\right|\right]\right\}
$$


Notando-se que:

$$
\frac{\sigma_{3}}{\sigma_{2}^{\frac{3}{2}}}=\frac{\mathrm{N}}{\mathrm{N}^{3}}\left(\frac{\mathrm{N}}{\mathrm{N}^{2}}\right)^{\frac{-3}{2}}=\frac{1}{\sqrt{\mathrm{N}}}=\mathrm{N}^{\frac{-1}{2}}
$$

encontra-se:

$$
\mathrm{P}=1 / 2+1 / 2\left\{\operatorname{tgh}\left[\mathrm{N}^{-1 / 2}\left|\mathrm{E}_{\mathrm{hk} l} \mathrm{E}_{\mathrm{h} \cdot \mathrm{k},}, \mathrm{E}_{\mathrm{h}-\mathrm{h}^{\prime}, \mathrm{k}-\mathrm{k}^{\prime}, H^{\prime}}\right|\right]\right\}
$$

As equações 161 e 163 são as de maior uso devido às peculiaridades com que são tratadas no cálculo dos $\mathrm{E}$ as classes especiais de reflexões.

Finalmente, pode-se escrever as equações de probabilidade em termos de fatores de estrutura unitário e fatores de estrutura normalizados, para o caso particular onde $\mathrm{s}(2 \mathrm{~h}, 2 \mathrm{k}, 2 l) \approx \mathrm{s}(\mathrm{hkl}) \cdot \mathrm{s}(\mathrm{hkl})$

$$
\mathrm{P}_{+}\left(\mathrm{U}_{2 \mathrm{~h}, 2 \mathrm{k}, 2 l}\right)=1 / 2+1 / 2\left\{\operatorname{tgh}\left[\left(\sigma_{3} / 2 \sigma_{2}^{3}\right)\left|\mathrm{U}_{2 \mathrm{~h}, 2 \mathrm{k}, 2 !}\right|\left(\mathrm{U}_{\mathrm{hk} l}^{2}-\sigma_{2}\right)\right]\right\}
$$

ou

$$
\mathrm{P}_{+}\left(\mathrm{E}_{2 \mathrm{~h}, 2 \mathrm{k}, 2 !}\right)=1 / 2+1 / 2\left\{\operatorname{tgh}\left[\left(\sigma_{3} / 2 \sigma_{2}^{3 / 2}\right)\left|\mathrm{E}_{2 \mathrm{~h}, 2 \mathrm{k}, 2 l}\right|\left(\mathrm{E}_{\mathrm{hkll}}^{2}-1\right)\right]\right\}
$$

onde $\mathrm{P}_{+}\left(\mathrm{U}_{\mathrm{hkl}}\right)$ é a probabilidade que $\mathrm{U}_{2 \mathrm{~h}, 2 \mathrm{k}, 2 l}$ tenha sinal positivo.

Frequentemente ocorre que nos últimos estágios de determinação das fases existe um grande número de relações na forma da equação 154 para uma mesma reflexão, cada uma delas com probabilidade não muito grande, de forma que aumenta-se a confiabilidade usando a relação conhecida como $\Sigma_{2}$ :

$$
\mathrm{s}(\mathrm{hkl})=\sum_{h^{\prime} k^{\prime} l^{\prime}} \mathrm{s}\left(\mathrm{h}^{\prime} \mathrm{k}^{\prime} l^{\prime}\right) \mathrm{s}\left(\mathrm{h}-\mathrm{h}^{\prime}, \mathrm{k}-\mathrm{k}^{\prime}, l-l^{\prime}\right)
$$


Sob estas condições

$$
\begin{aligned}
& \mathrm{P}_{+}=1 / 2+1 / 2\left\{\operatorname{tgh}\left[\mathrm{N}\left|\mathrm{U}_{\mathrm{hk} l}\right| \sum_{h^{\prime} k^{\prime} \prime^{\prime}} \mathrm{U}_{\mathrm{h}^{\prime} \mathrm{k}^{\prime} \eta}, \mathrm{U}_{\mathrm{h}-\mathrm{h}^{\prime}, \mathrm{k}-\mathrm{k}^{\prime}, I-r^{\prime}}\right]\right\}
\end{aligned}
$$

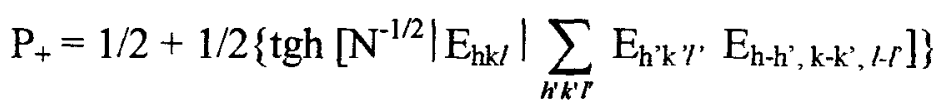

Uma vez que o argumento da tangente hiperbólica pode ser positivo ou negativo, valores de $\mathrm{P}_{+}$menores que $1 / 2$ indicam que o sinal da fase é negativo, enquanto que valores maiores que $1 / 2$ indicam sinal positivo, assim:

$$
\mathrm{P}_{-}=1-\mathrm{P}_{+}
$$

\subsubsection{2 - MÉTODOS NÃO CENTROSSIMÉTRICOS}

Para o caso de grupos espaciais não centrossimétricos a equação 166 é escrita na seguinte forma:

$$
\phi(h) \approx \phi\left(h^{\prime}\right)+\phi\left(h^{\prime} h^{\prime}\right)
$$

ou

$$
\phi(h)+\phi\left(h^{\prime}\right)+\phi\left(\bar{h}-\overline{h^{\prime}}\right)=\phi_{3} \approx 0
$$

onde $\phi$ é o ângulo da fase expressado em frações de um ciclo, e como no caso anterior a probabilidade de $\phi_{3}=0$ aumenta com a magnitude das reflexões envolvidas [Cochran, 1955]. 
As fases agora podem assumir diversos valores, então as probabilidades assumirão a forma de uma distribuição que fornecerão vários graus de erro, assim para a equação 170, a distribuição da probabilidade será dada por [Karle e Hauptman, 1950]:

$$
\mathrm{P}\{\Delta \phi(\mathbf{h})\}=\frac{\mathrm{e}^{\left.\mathrm{K}\left(\mathbf{h}, \mathbf{h}^{\prime}\right) \cos \{\phi h)-\left[\phi\left(\mathbf{h}^{\prime}\right) \phi\left(\mathbf{h}-\mathbf{h}^{\prime}\right)\right]\right\}}}{2 \pi \mathbf{d}_{0}\left[\mathrm{~K}\left(\mathbf{h}, \mathbf{h}^{\prime}\right)\right]}
$$

onde

$$
\mathrm{K}\left(\mathbf{h}, \mathbf{h}^{\prime}\right)=2\left(\mathrm{~N}^{-1 / 2}\right)\left|\mathrm{E}(\mathbf{h}) \mathrm{E}\left(\mathbf{h}^{\prime}\right) \mathrm{E}\left(\overline{\mathbf{h}} \overline{\mathbf{h}^{\mathbf{\prime}}}\right)\right|
$$

e, $I_{n}$ é a função de Bessel modificada.

Como exemplo a figura 20 exibe a expressão para a probabilidade calculada para os diferentes valores de $\mathrm{K}$.

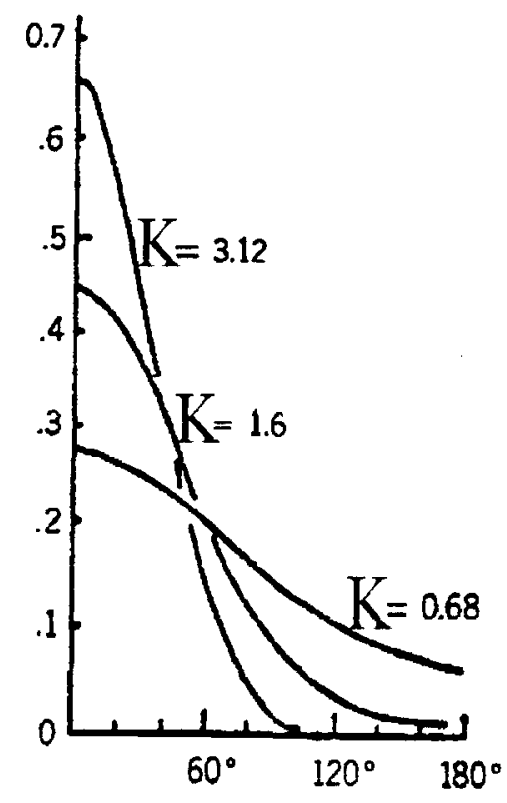

Figura 20- Distribuição de probabilidades de $\phi_{3}$ para três valores de $K$.

A tabela 2 mostra os valores médios de $\mathrm{E}$ para diferentes números de átomos na cela e os diferentes valores de $\mathrm{E}$. 
Tabela 2

VALORES DE K E E PARA DIFERENTES NÚMEROS DE ÁTOMOS

\begin{tabular}{|c|c|c|}
\hline \multicolumn{2}{|c|}{$<\mathrm{E}>=\left(\mathrm{E}_{1} \mathrm{E}_{2} \mathrm{E}_{3}\right)^{1 / 3}$} \\
\hline $\mathrm{K}$ & $\mathrm{N}=100$ & $\mathrm{~N}=30$ \\
\hline 0,68 & 1,5 & 1,2 \\
\hline 1,60 & 2,0 & 1,6 \\
\hline 3,12 & 2,5 & 2,0 \\
\hline
\end{tabular}

De uma forma particular, onde existem mais do que um apontamento triplo para a fase de uma reflexão, pode-se escrever a equação desenvolvida por Karle e Hauptman [1950], conhecida como fórmula da tangente:

$$
\operatorname{tg}[\phi(\mathbf{h})]=\frac{\sum_{\mathbf{h}^{\prime}} K\left(\mathbf{h}, \mathbf{h}^{\prime}\right) \operatorname{sen}\left[\phi\left(\mathbf{h}^{\prime}\right)+\phi\left(\mathbf{h}-\mathbf{h}^{\prime}\right)\right]}{\sum_{\mathbf{h}^{\prime}} K\left(\mathbf{h}, \mathbf{h}^{\prime}\right) \cos \left[\phi\left(\mathbf{h}^{\prime}\right)+\phi\left(\mathbf{h}-\mathbf{h}^{\prime}\right)\right]}
$$

A fórmula da tangente tem grande importância prática em métodos diretos para cristais não centrossimétricos, é frequentemente utilizada na forma pesada, para a qual as contribuições para as somas são pesadas em termos da probabilidade das relações [Hull e Irwin, 1978].

A probabilidade de distribuição de $\phi(\mathbf{h})$ predita pela equação 174 pode ser calculada de equações similares a equação 172 , mas é frequentemente descrita em termos de sua variança, o quadrado dos seus desvios padrão. 


\section{8 - ADIC̄ÃO SIMBÓLICA}

A adição simbólica foi o primeiro método direto utilizado como rotina na determinação de estruturas cristalinas [Karle e Karle, 1966].

Através desta técnica, obtêm-se apenas um único conjunto de fase através da relação: $\phi(\mathbf{h}) \approx \phi(\mathbf{k})+\phi(\mathbf{h}-\mathbf{k})$

Assumindo esta aproximação e considerando-a igual a zero, tem-se que se forem conhecidas duas fases é possível calcular a terceira. Isto pode ser conseguido através da fixação da origem, mas no caso das reflexões que fixam a origem não serem suficientes, combinam-se outras reflexões com fases desconhecidas, que são representadas por símbolos e então novas fases são calculadas em termos desses símbolos. Posteriormente calcula-se o valor destas fases atribuindo aos símbolos valores de fase adequados.

Após a substituição dos símbolos, refina-se essas fases através da fórmula da tangente (equação 174) e assim é possível encontrar as fases restantes obtendo assim, o conjunto de fases para o cálculo do mapa de densidade eletrônica usando como coeficientes os fatores de estrutura normalizado com as fases obtidas.

O método de adição simbólica inicia-se com uma coleção de n relações de fase para $\mathrm{m}$ fases desconhecidas onde $\mathrm{m}<<\mathrm{n}$. Esta reflexões iniciais são escolhidas de forma que em princípio todas as outras fases podem ser obtidas a partir delas.

Quanto ao procedimento uma adição simbólica pode-se estabelecer como passos:

a - Cálculo dos fatores de estrutura normalizados; 
b - Geração das relações de fase (tripletes);

c -Escolha do conjunto inicial ( fixação da origem);

d- Escolha das reflexões usadas com fase igual a símbolo;

e- Determinação de valores numéricos aproximados dos símbolos;

f- Refinamento numérico das fases;

g- Cálculo das sínteses de Fourier e suas interpretações;

h- Rápido refinamento da estrutura.

\subsection{9 - AS BASES DO MÉTODO DE MULTISSOLUCÃO}

Embora a adição simbólica seja um dos importantes métodos diretos [Karle e Karle, 1964] existe um problema com este método, que é o fato de não ser possível combinar certas indicações de fases se elas envolverem diferentes símbolos. Assim, por exemplo, se a fase está indicada como $a+b$ em uma relação de fase e c+d em uma outra relação, então não será permitida uma indicação combinada como uma média $(a+b+c+d) / 2$, Isto ocorre devido a ambiguidade de " $2 \pi$ " na definição das fases. Então com $a=b=\pi / 2$ e $c=d=-\pi / 2$ as duas indicações individuais serão as mesmas, desde que $\pi \equiv-\pi$, mas a indicação média deve ser zero.

Nos estágios iniciais da determinação de fases existem usualmente muitas indicações de fases em que todas podem ser usadas em um modo positivo, por esta razão 
Germain e Woolfson [1968] propuseram o uso de valores de fases explícitos, melhores então do que símbolos, e estas idéias originaram o sistema de programas MULTAN (multisolution tangent formula) e outras técnicas similares.

Os métodos de multissolução, são de variedades diferentes, mas todos sistematizam o mesmo processo de determinação de fase, que ao invés de utilizar um único valor para o símbolo (como na adição simbólica) utilizam um conjunto de valores possíveis de fase para esse símbolo, isto é, estabelecem um intervalo de valores numéricos que são usados. Para cada conjunto de fases utiliza-se a fórmula da tangente, ou uma forma otimizada desta [Hull e Irwin, 1978] para o cálculo das fases do restante das reflexões. Assim para cada conjuto obtem-se os valores das fases, relativos aos valores iniciais atribuidos aos símbolos, gerando-se conjuntos de fases, ou soluções possiveis.

A avaliação da confiabilidade é feita pelo cálculo de valores que representam a consistência entre os dados observados e o modelo que são as figuras de mérito.

As principais etapas na aplicação do método são:

1 - Cálculo dos módulos dos fatores de estrutura normalizados.

Nesta etapa se alguma informação sobre a geometria molecular é conhecida pode-se melhorar os cálculos dos fatores de estrutura normalizados incorporando esta informação.

2 - Seleção de um conjunto de reflexões com os maiores valores de E.

Neste estágio seleciona-se as reflexões com maiores valores de $|E|$, através das quais a estrutura pode ser resolvida (origem, enantiomorfo, reflexões adicionais para as quais vai ser variada a fase). O número de reflexões a serem selecionadas depende do número 
de átomos da molécula e do sistema cristalino. Uma vez que ao final do processo deverá ser calculado um mapa de densidade eletrônica. No sistema MULTAN, a rotina estabelece este número como sendo $4 \times \mathrm{N}+100$, onde $\mathrm{N}$ é o número de átomos da unidade assimétrica, $\mathrm{e}$ acrescenta a ele mais 100 reflexões para o sistema triclínico, e 50 para o sistema monoclínico.

3 - Geração das relações $\Sigma_{2}$ (tripletes):

As relações $\Sigma_{2}$ são geradas pelo uso de reflexões com os maiores valores de $|E|$ e também pelos pares de contribuições das reflexões de menores valores de $|\mathrm{E}|$.

4- - Seleção de um conjunto de partida:

O algorítmo para a escolha de um arranjo de partida é chamado de procedimento de convergência descrito por Germain, Main e Woolfson [1970], cujo diagrama de fluxo está representado na figura 21.

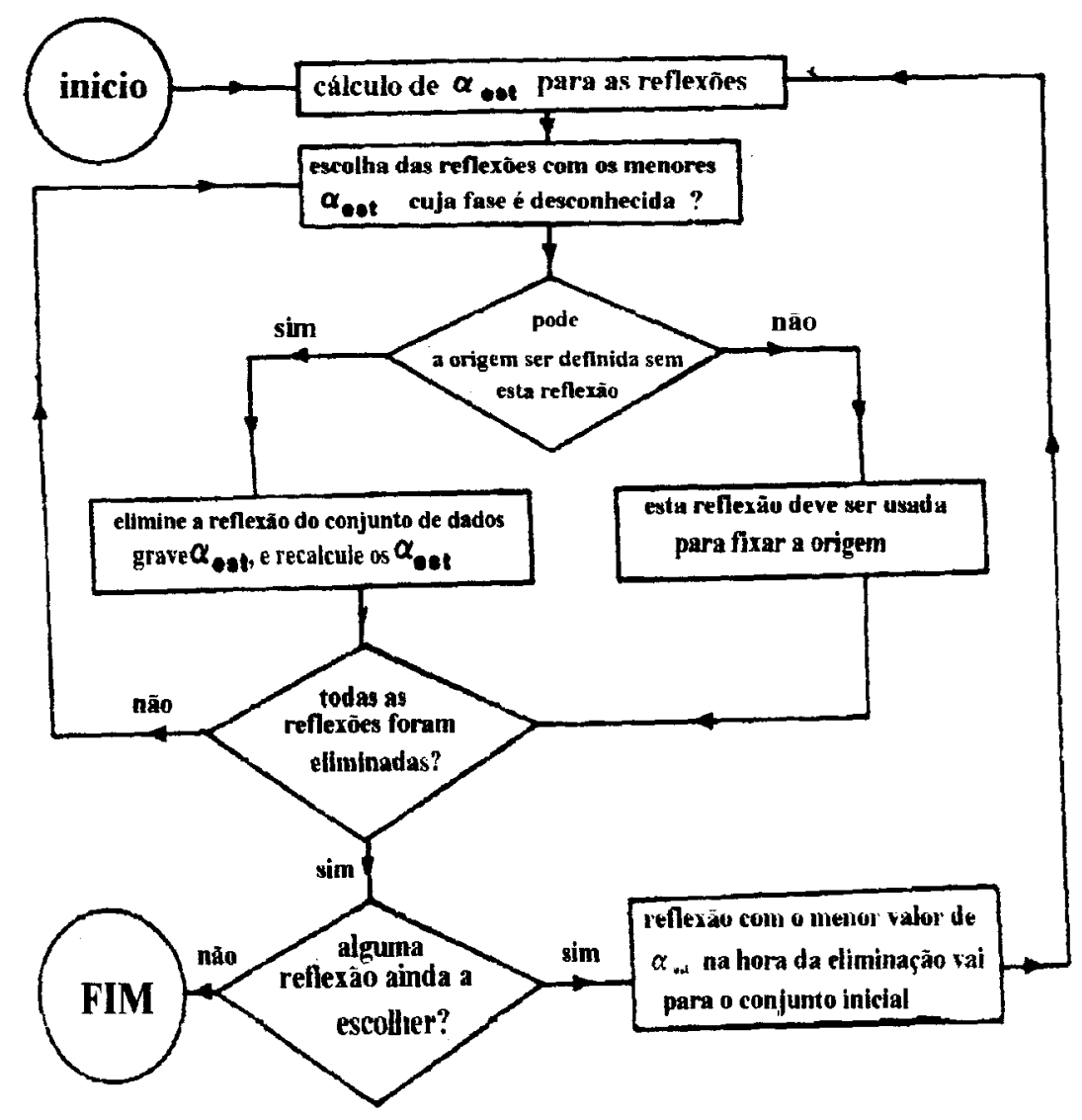

Figura 21 - Diagrama do processo de convergência. 
O valor de $\alpha_{\text {est }}$ é encontrado a partir da equação:

$$
\alpha_{e s t}^{2}=\sum_{\mathbf{k}} \mathrm{K}(\mathbf{h}, \mathbf{k})^{2}+\sum_{\mathbf{k}} \sum_{l} \mathrm{~K}(\mathbf{h}, \mathbf{k}) \mathrm{K}(\mathbf{h}, \boldsymbol{l}) \frac{\mathbf{I}_{1}\{\mathrm{~K}(\mathbf{h}, \mathbf{k})\}}{\mathbf{I}_{0}\{\mathrm{~K}(\mathbf{h}, \mathbf{k})\}} \frac{\mathbf{I}_{1}\{\mathrm{~K}(\mathbf{h}, \boldsymbol{l})\}}{\mathbf{I}_{0}\{\mathrm{~K}(\mathbf{h}, \boldsymbol{l})\}}
$$

onde $\mathbf{I}_{0}$ e $\mathbf{I}_{1}$ são funções de Bessel, a somatória dupla sobre $\mathbf{k}$ e $\boldsymbol{l}$ presupõe $\mathbf{k} \neq \boldsymbol{l}$.

A cada estágio o algoritmo de convergência identifica aquelas reflexões de $\alpha_{e s t}$ que são elimindas do processo desde que elas não sejam necessárias no processo de fixação da origem. Quando $\alpha_{e s t}$ é igual a zero isto significa que esta reflexão não pode ter sua fase determinada pelas remanescentes, e neste caso essa reflexão é incorporada ao conjunto de partida e sua fase vai ser variada no processo de geração dos conjuntos de soluções possíveis.

5- Atribuição das fases iniciais

O processo de determinação de fase através da formula da tangente inicia-se com as fases que definem a origem e enantiomorfo, e mais um conjunto de fases atribuídas em valores permitidos, para fornecer diferentes conjuntos de partida.

6 - Geração dos conjuntos de soluções possiveis.

Através da aplicação da formula da tangente é possivel calcular as fases para os diferentes conjuntos iniciais, e também refina-las.

7 - Avaliação da confiabilidade das soluções obtidas.

Uma vez que as fases de todos os conjuntos foram calculadas, a avaliação da confiabilidade de cada conjunto é feita pelo cálculo das figuras de méritos.

Alguns exemplos de figuras de mérito utilizadas são: 
Figura de Mérito Absoluta [Woolfson,1976]

Esta figura de mérito utiliza diferentes valores de $\alpha$, através de:

$$
\text { ABSFOM }=\frac{\sum_{\mathbf{h}}\left\{\alpha(\mathbf{h})-\alpha_{r}(\mathbf{h})\right\}}{\sum_{\mathbf{h}}\left\{\alpha_{e s t}(\mathbf{h})-\alpha_{r}(\mathbf{h})\right\}}
$$

onde:

$$
\begin{gathered}
\alpha(\mathbf{h})=\left\{\left[\Sigma_{\mathbf{n}} K(\mathbf{h}, \mathbf{k}) \cos [\phi(\mathbf{h})+\phi(\mathbf{h}-\mathbf{k})]\right]^{2}+[\Sigma K(\mathbf{h}, \mathbf{k}) \operatorname{sen}[\phi(\mathbf{k})+\phi(\mathbf{h}-\mathbf{k})]]^{2}\right\}^{1 / 2} \\
\alpha_{e s t}=\sum_{\mathbf{h}} \mathrm{K}(\mathbf{h}, \mathbf{k}) \frac{\mathbf{I}_{1}\{\mathrm{~K}(\mathbf{h}, \mathbf{k})\}}{\mathrm{I}_{0}\{\mathrm{~K}(\mathbf{h}, \mathbf{k})\}}
\end{gathered}
$$

e $\alpha_{\mathrm{r}}$ é dado por:

$$
\alpha_{r}=\sum_{\mathbf{h}} \sqrt{\sum_{\mathbf{h}} K^{2}(\mathbf{h}, \mathbf{k})}
$$

O valor de ABSFOM varia entre 0 (zero), para fases aleatórias e 1 (um) para o caso em que os valores de $\alpha$ são iguais aos valores esperados.

\section{Figura de mérito $\Psi_{0}$}

A segunda figura de mérito $\Psi_{0}$ é calculada usando-se os maiores e menores valores de $|E|$, cujas fases foram determinadas e é calculado por:

$$
\Psi_{0}=\frac{\sum_{\mathbf{h}}\left|\sum_{\mathbf{k}} E(\mathbf{k}) E(\mathbf{h}-\mathbf{k})\right|}{\sum_{\mathbf{h}}\left(\sum_{\mathbf{k}}|E(\mathbf{k}) E(\mathbf{h}-\mathbf{k})|^{2}\right)^{1 / 2}}
$$


Para os conjuntos de fases apropriadas, ou proximas da realidade o valor de $\Psi_{0}$ tende a unidade.

\section{Figura de mérito $R$.}

A terceira figura de mérito é o índice R (fator de concordância) [Karle e Karle 1966]. Esta figura de mérito na realidade fornece o fator de discordância entre a estrutura proposta e verdadeira, portanto para valores menores de $\mathrm{R}$ melhor será o modelo de estrutura encontrado.

O Indice R é definido da seguinte forma:

$$
R=\frac{\left.\sum_{\mathbf{h}}|| E(\mathbf{h})\right|_{\mathrm{obs}}-|E(\mathbf{h})|_{\mathrm{calc}} \mid}{\sum_{\mathbf{h}}|\mathrm{E}(\mathbf{h})|_{\mathrm{obs}}}
$$

onde, $|E(h)|_{\text {obs são os módulos de fatores de estrutura normalizados observados e }|E(\mathbf{h})|_{\text {calc }}}$ são os módulos dos fatores de estrutura calculados de acordo com:

$$
|E(\mathbf{h})|_{\text {cale }}=K \sum_{\mathbf{k}} E(\mathbf{k}) E(\mathbf{h}-\mathbf{k})
$$

onde $\mathrm{K}$ é uma constante de normalização garantindo que:

$$
\sum_{\mathbf{h}}|\mathrm{E}(\mathbf{h})|_{\mathrm{obs}}^{2}=\sum_{\mathbf{h}}|\mathrm{E}(\mathbf{h})|_{\mathrm{calc}}^{2}
$$


Uma outra forma da figura de mérito $\mathrm{R}$ é a calculada usando-se os valores de $\alpha$, conhecida como R $\alpha$ que então tem a seguinte forma:

$$
\mathrm{R} \alpha=\frac{\sum_{\mathbf{h}}\left|\alpha(\mathbf{h})-\alpha_{\mathrm{est}}(\mathbf{h})\right|}{\sum_{\mathbf{h}} \alpha_{\mathrm{est}}(\mathbf{h})}
$$

O que ocorre normalmente, é que na maioria das vezes as três ou quatro figuras de mérito descritas não são satisfeitas ao mesmo tempo para os conjuntos considerados. Assim para solucionar o problema definiu-se uma figura de mérito combinada envolvendo ABSFOM, $\Psi_{0}$ e R que foi chamada de CFOM, expressa da forma:

$\mathrm{CFOM}=w_{1} \frac{\mathrm{ABSFOM}-(\mathrm{ABSFOM})_{\min }}{(\mathrm{ABSFOM})(\mathrm{ABSFOM})_{\max }}+w_{2} \frac{\left(\Psi_{0}\right)_{\max }-\Psi_{0}}{\left(\Psi_{0}\right)_{\max }-\left(\Psi_{0}\right)_{\min }}+w_{3} \frac{\mathrm{R}_{\max }-\mathrm{R}}{\mathrm{R}_{\max }-\mathrm{R}_{\min }}$

ou usando $R \alpha$ :

$\mathrm{CFOM}=w_{1} \frac{\mathrm{ABSFOM}-(\mathrm{ABSFOM})_{\min }}{(\mathrm{ABSFOM})(\mathrm{ABSFOM})_{\max }}+w_{2} \frac{\left(\Psi_{0}\right)_{\max }-\Psi_{0}}{\left(\Psi_{0}\right)_{\max }-\left(\Psi_{0}\right)_{\min }}+w_{3} \frac{\mathrm{R} \alpha_{\max }-\mathrm{R} \alpha}{\mathrm{R} \alpha_{\text {max }}-\mathrm{R} \alpha_{\text {min }}}$

onde $\mathbf{w}_{1}, \mathbf{w}_{2}, \mathrm{w}_{3}$ são pesos geralmente tomados como $0,6,1,2$ e 1,2 respectivamente, para dar a CFOM valores no intervalo 0 a 3 .

\section{7 - Mapas de E.}

Mapa de E é o nome dado aos mapas de densidade eletrônica, calculados usando-se como coeficientes da série de Fourier os valores dos módulos dos fatores de estutura normalizados, $|E|$, atribuindo a eles as fases calculadas pelo processo descrito anteriormente. 
Desta forma finalmente constrói-se os mapas de Fourier das melhores soluções. Nesta etapa quase ou todos os átomos são localizados. O modelo inicial da estrutura é assim obtido estando terminada a etapa de aplicação dos métodos diretos.

Caso o modelo obtido seja muito incompleto, ou seja, poucos átomos foram localizados, pode-se reiniciar o processo de obtenção das fases, usando como informação, para o cálculo dos fatores de estrutura normalizados, a parte da estrutura obtida no modelo. $\mathrm{O}$ processo restante segue as etapas descritas.

\subsection{2 - REFINAMENTO DE UMA ESTRUTURA}

Após resolver o problema de encontrar as fases obtem-se um modelo que consiste em uma coleção de parâmetros especificando as localizações de todos os átomos (ou da maior parte) dentro da cela unitária. O modelo obtido deve agora ser ajustado (refinado) relativamente às intensidades das reflexões medidas, ou seja, no estágio de refinamento de uma estrutura a ser analisada, supõe-se que a estrutura contém todos os átomos e a apartir daí, pode-se localizar os átomos restantes ou ajustar a posição dos já encontrados, até o máximo de concordância permitido pelos dados coletados.

\subsection{1 - REFINAMENTO PELA SÍNTESE DE FOURIER DIFERENÇA}

Uma das maneiras de encontrar-se os átomos, não considerados no modelo, em uma cela unitária é uma aproximação importante, conhecida como síntese de Fourier diferença (Stout e Jensen, 1989), na qual calcula-se a densidade eletrônica ( $\rho(x y z)$ ) usando 
como coeficientes da série as diferenças entre os módulos dos fatores de estrutura observado e calculado $\left(\Delta \mathrm{F}=\left|\mathrm{F}_{\mathrm{o}}\right|-\left|\mathrm{F}_{\mathrm{c}}\right|\right)$.

Escrevendo separadamente $\rho_{\mathrm{o}}$ e $\rho_{\mathrm{c}}$, densidade eletrônica observada e calculada respectivamente,

$$
\begin{aligned}
& \rho_{\mathrm{o}}(\mathrm{x}, \mathrm{y}, \mathrm{z})=\frac{1}{\mathrm{~V}} \Sigma \Sigma \Sigma \mathrm{F}_{\mathrm{o}}(\mathrm{hk} l) \mathrm{e}^{-2 \pi \mathrm{i}(\mathrm{hx}+\mathrm{ky}+l \mathrm{z})}+\mathrm{R} \\
& \rho_{\mathrm{c}}(\mathrm{x}, \mathrm{y}, \mathrm{z})=\frac{1}{\mathrm{~V}} \Sigma \Sigma \Sigma \mathrm{F}_{\mathrm{c}}(\mathrm{hk} l) \mathrm{e}^{-2 \pi \mathrm{i}(\mathrm{hx}+\mathrm{ky}+l \mathrm{z})}+\mathrm{R}^{\prime}
\end{aligned}
$$

onde R e R' são representações remanescentes das partes omitidas das séries, e tomando-se as duas séries e subtraíndo termo a termo encontra-se:

$$
\rho_{\mathrm{o}}(\mathrm{x}, \mathrm{y}, \mathrm{z})-\rho_{\mathrm{c}}(\mathrm{x}, \mathrm{y}, \mathrm{z})=\frac{1}{\mathrm{~V}} \Sigma \Sigma \Sigma\left(\mathrm{F}_{\mathrm{o}}-\mathrm{F}_{\mathrm{c}}\right)_{\mathrm{hk} l} \mathrm{e}^{-2 \pi \mathrm{i}(\mathrm{hx}+\mathrm{ky}+l \mathrm{z})}+\mathrm{R}-\mathrm{R}^{\prime}
$$

como as terminações da síntese são aproximadamentes iguais, ou seja, próximas de zero então a síntese de diferença pode ser escrita de uma nova forma:

$$
\rho_{o}-\rho_{c}=\frac{1}{V} \Sigma \Sigma \Sigma \Delta F(h k l) e^{-2 \pi i(h x+k y+l z)}
$$

ou separando $\mathrm{F}(\mathrm{hk} l)$ em amplitude e fase, a expressão 189 adquire uma nova forma, dada por:

$$
\Delta \rho=1 / \mathrm{V} \Sigma \Sigma \Sigma\left(\left|\mathrm{F}_{\mathrm{o}}\right|-\left|\mathrm{F}_{\mathrm{c}}\right|\right) \mathrm{e}^{\mathrm{i} \alpha \mathrm{c}} \mathrm{e}^{-2 \pi \mathrm{i}(\mathrm{hx}+\mathrm{ky}+\mathrm{lz})}
$$

onde $\alpha_{\mathrm{c}}$ é a fase do Fator de estrutura calculado. O significado desta expressão é que ela representa estritamente a diferença entre a densidade eletrônica real e a do modelo usado para o cálculo do fator de estrutura.

Uma outra vantagem da síntese de diferença é de mostrar claramente os erros posicionais dos átomos na estrutura que está sendo analisada, podendo então ser utilizada como uma bases para o posterior refinamento.

Os efeitos nas coordenadas posicionais pode ser mostrado através da figura 22. 

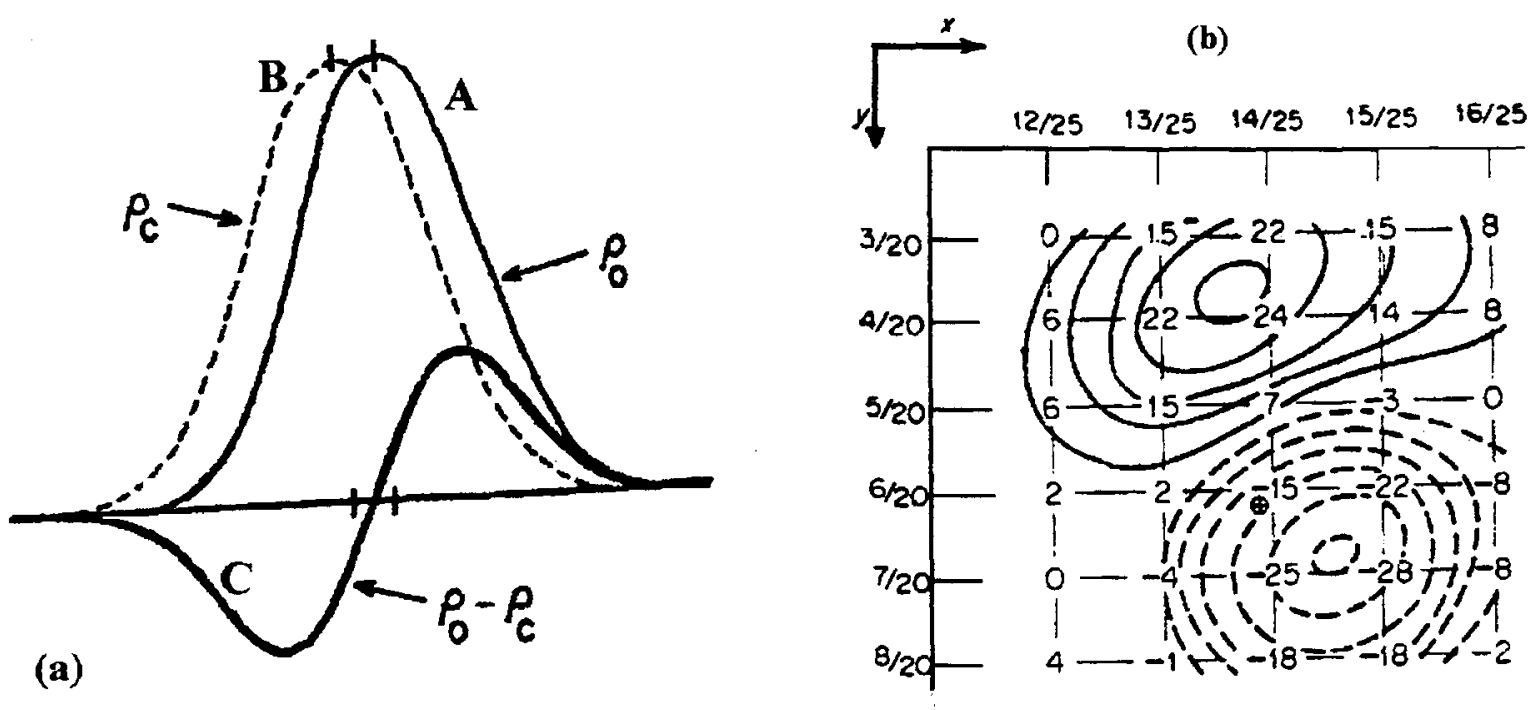

Figura 22 - Efeito dos erros posicionais visualizado na Fourier diferença. (a) na direção de $x$; (b) no plano xy.

$\mathrm{Na}$ figura 22a a linha A ilustra a densidade eletrônica ideal de um átomo, projetado sobre uma linha através do seu centro, a linha B (tracejada) ilustra a posição do átomo do modelo usado no cálculo de $\mathrm{F}_{\mathrm{c}}$ e finalmente a linha $\mathrm{C}$ é o resultado da síntese $\Delta \mathrm{F}$.

A figura $22 \mathrm{~b}$ mostra um átomo mal localizado, linhas tracejadas (região negativa) e a posição correta do átomo está representada na região de linha contínua.

Torna-se evidente que um erro em uma coordenada resultará em um gradiente na sintese $\Delta \mathrm{F}$, assim, a correção para uma coordenada atômica estará na direção do gradiente que se direcionará a uma região mais positiva.

Correções nas coordenadas $\mathrm{x}$, por exemplo, podem ser obtidas com suficiente precisão através da expressão:

$$
\Delta_{\mathrm{xq}}=\frac{\text { inclinação }}{\text { curvatura }}=\frac{\partial \Delta \rho / \partial x_{q}}{\partial^{2} \rho / \partial x_{q}^{2}}
$$

Equações similares podem ser obtidas para as coordenadas y e $z$.

Os valores próprios para as curvaturas podem ser derivados de uma síntese de $\mathrm{F}_{\mathrm{o}}$, contendo somente termos correspondentes àqueles usados na síntese de $\Delta \mathrm{F}$ 
Na prática entretanto esse método de ajuste, das coordenadas posicionais dos átomos do modelo, demanda um grande volume computacional, sendo este tipo de ajuste pouco utilizado.

$\mathrm{Na}$ equação 191, no primeiro termo da exponencial, aparece o termo $\alpha_{\mathrm{c}}$ (fase calculada), mostrando que a principal informação obtida é que se o valor de $\alpha_{c}$ for igual ou próximo a $\alpha_{0}$ (fase do fator de estrutura observado), resultará o fornecimento de uma medida direta dos erros entre o modelo usado $\left(\left|F_{c}\right|\right)$ e a verdadeira estrutura, implícito pelos valores de $\left|F_{o}\right|$. A consequência importante é que através deste tipo de síntese pode-se localizar átomos não incluidos no modelo, permitindo completar a estrutura.

O principal argumento para estas considerações vem das considerações feitas para as situações limitantes.

\subsubsection{1 - SITUAÇÕES LIMITANTES DA FOURIER DIFERENÇA}

Deve-se analisar agora os casos limitantes de $\left|F_{n}\right|$ e $\left|F_{c}\right|$, que são basicamente $\left|F_{o}\right| \approx\left|F_{c}\right|$ e $\left|F_{o}\right| \neq\left|F_{c}\right|$

Para o segundo caso duas situações diferentes podem ocorrer, $\left|F_{o}\right|>\left|F_{c}\right|$ e $\left|F_{c}\right|>\left|F_{o}\right|$. Os três casos estão mostradas na figura 23 .

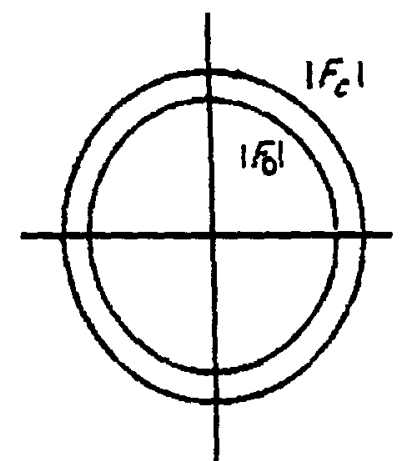

(a)

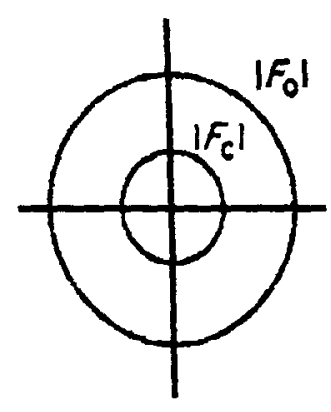

(b)

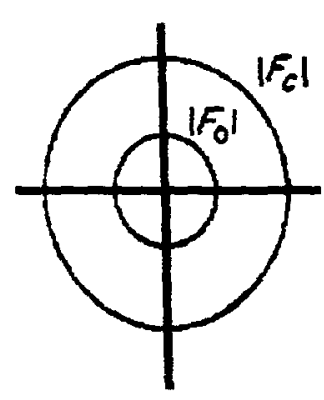

(c)

Figura 23 - Situações limites da sintese $\Delta F$. (a) $\left|F_{o}\right| \approx\left|F_{c}\right| ;$ (b) $\left|F_{0}\right|>\left|F_{c}\right|$; (c) $\left|\mathbf{F}_{\mathbf{o}}\right|>\left|\mathbf{F}_{\mathbf{c}}\right|$. 
Primeiro caso onde $\left|F_{n}\right| \approx\left|F_{c}\right|$ :

Se o modelo de fases for correto existirá alta probabilidade de que $\alpha_{c}$ seja aproximadamente correspondente ao valor da fase verdadeira de $F_{n}$. É portanto nesta suposição que a síntese $F_{n}$ (sintese de Fourier usando como coeficientes $\left|F_{n}\right|$ com a fase igual a $\alpha_{c}$ ) está baseada.

Para o caso em que $\alpha_{c}$ difere do valor verdadeiro, um valor alto de $\left|F_{o}\right|$ introduzirá sérios erros na síntese resultante.

Reflexões nas quais $\left|F_{o}\right| \approx\left|F_{c}\right|$ (figura 23a) tendem a reproduzir o modelo, e acrescentam apenas pequenas informações, desta forma o uso desta reflexões oferece, neste estágio, pequeno ganho, mas possíveis riscos de grandes distorções. $\mathrm{Na}$ síntese de Fourier diferença entretanto $\left|F_{n}\right|-\left|F_{c}\right| \approx 0$, então o efeito destas reflexões é automaticamente minimizado.

Segundo caso $\left|F_{n}\right| \neq\left|F_{c}\right|$ :

Para este caso duas situações se apresentam, $\left|F_{n}\right|>\left|F_{c}\right|$ e $\left|F_{c}\right|>\left|F_{0}\right|$.

No caso onde $\left|F_{n}\right|>\left|F_{c}\right|$ (figura 23b, então $\left|F_{n}\right|$ fará uma importante contribuição para a série de Fourier diferença e proporcionará informações úteis; desde que $\alpha_{\mathrm{c}}$ seja próximo de $\alpha_{0}$. Entretanto a probabilidade de correspondência entre $\alpha_{\mathrm{c}}$ e $\alpha_{0}$, é tanto menor quanto maior for a diferença entre $\left|F_{o}\right|$ e $\left|F_{c}\right|$ e diminuirá ainda mais quando $\left|F_{c}\right|$ se aproxima de zero, desta forma estas reflexões são inseguras.

Quando $\left|F_{c}\right|>\left|F_{o}\right|$ (figura 23c) embora as reflexões observadas transportem informações sobre a estrutura verdadeira, o módulo $\left|F_{0}\right|$ contribue com muito pouco efeito na somatória no caso da sintese de Fourier com os $\mathrm{F}_{\mathrm{o}}$ como coeficientes. 
Na sintese $\Delta F$ entretanto, ||$F_{o}|-| F_{c}||$ serão grandes e farão uma importante contribuição para a síntese.

Deve ser lembrado porém que estes termos, ||$F_{o}|-| F_{c}||$, terão suas fases mais ou menos corretas, dependendo da diferença entre $\alpha_{c}$ e $\alpha_{o}$.

A verdade sobre esta afirmação pode ser verificada pela interpretação da figura 24.

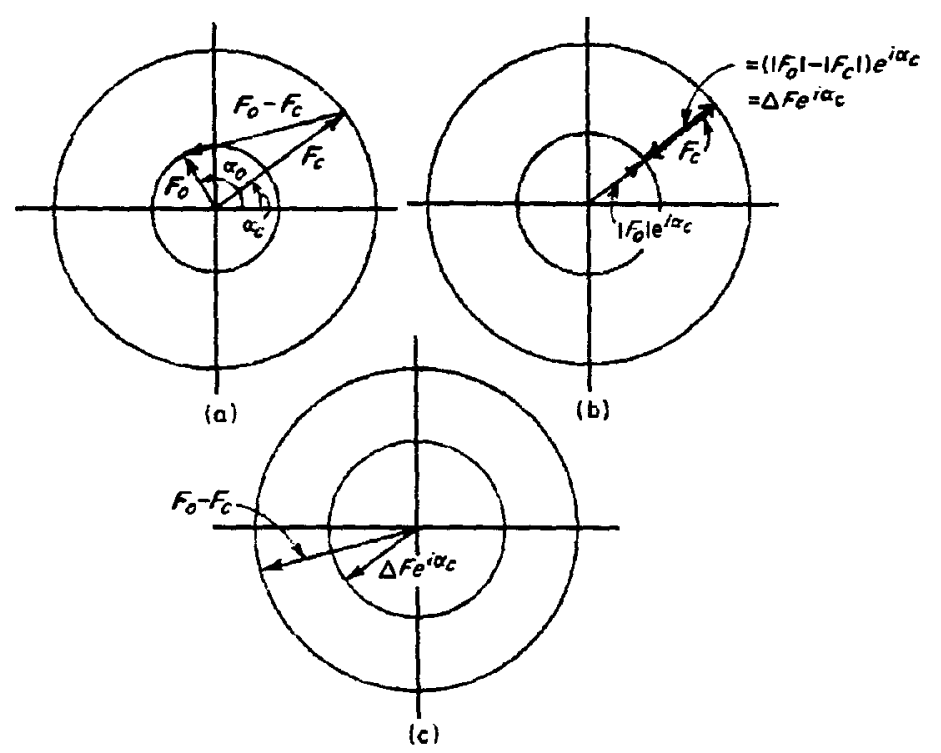

Figura 24 - (a) vetores para o caso $\left|F_{c}\right|>\left|F_{o}\right|$. (b) vetores para o caso, $\left|F_{c}\right|>\left|F_{o}\right|$.com $\alpha_{0}=\alpha_{c}$ (c) comparação entre os vetores $F_{o}-F_{c}$ e $\Delta F \mathrm{e}^{-i \alpha c}$.

Representando-se os coeficientes da síntese diferença $\left(\Delta F=F_{0}-F_{\mathbf{c}}\right)$, através de vetores para os três casos anteriores, ilustrados na figura 24 , pode -se escrever:

$$
|\Delta F| e^{i \alpha \Delta}=\left|F_{o}\right| e^{i \alpha o}-\left|F_{c}\right| e^{i \alpha c}
$$

Pela observação da figura $24 a$, nota-se que estes valores são justamente os vetores requeridos para corrigir o valor de $F_{c}$ para os valores reais de $F_{0}$. Estes vetores são os fatores resultantes da adição da densidade eletrônica quando necessária e subtração quando em excesso.

Como $\alpha_{0}$ é desconhecido, pode-se fazer as seguintes suposições: 


$$
\begin{aligned}
& \alpha_{o} \approx \alpha_{c} \\
& |\Delta F| e^{i \alpha\rfloor} \approx\left|F_{o}\right| e^{i \alpha c}-\left|F_{c}\right| e^{i \alpha c} \\
& |\Delta F| e^{i \alpha \Delta} \approx\left(\left|F_{o}\right|-\left|F_{c}\right|\right) e^{i \alpha c} \\
& |\Delta F| e^{i \alpha \Delta} \approx|\Delta F| e^{i \alpha c}
\end{aligned}
$$

Como considerou-se até o presente momento somente reflexões para as quais $\Delta F$ resulta sempre negativo a aproximação na realidade é:

$$
|\Delta F| \mathrm{e}^{\mathrm{i} \alpha \Delta} \approx-|\Delta F| \mathrm{e}^{\mathrm{i} \alpha c}
$$

Observando-se a figura $24 \mathrm{c}$, verifica-se que estes dois vetores são comparáveis em magnitude e fase.

Pode-se agora generalizar a construção geométrica destes fatores (equação 197) reescrevendo-a na seguinte forma:

$$
\Delta \mathrm{F}=-\mathbf{F}_{\mathbf{c}}+\mathbf{F}_{\mathbf{o}}
$$

Esta generalização pode ser ilustrada na figura 25 , concluindo-se que o valor de $\Delta F$ sempre deve estar situado sobre um círculo de raio $\left|F_{0}\right|$ ao redor dos $F_{c}$.

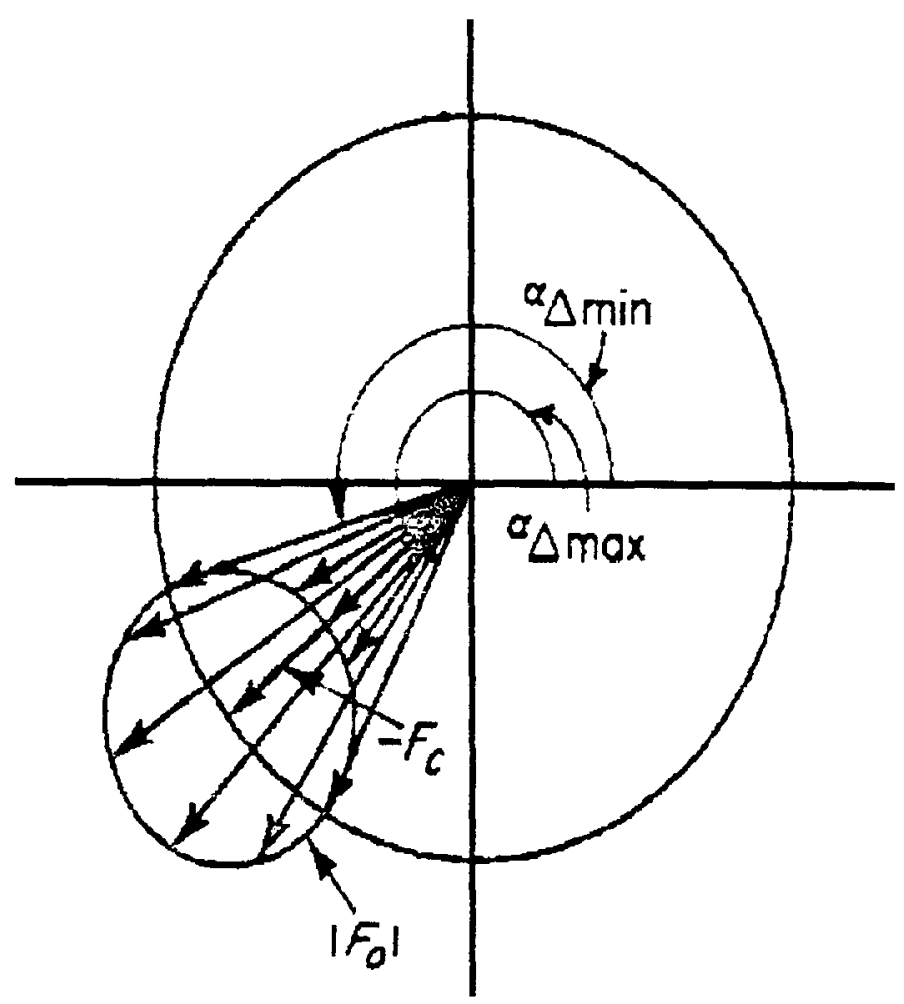

Figura 25 - Construção mostrando o intervalo de $\alpha$. 
A síntese $\Delta F$ também pode mostrar os efeitos dos erros em parâmetros térmicos usados no modelo.

Observando-se a figura 26 verifica-se que a linha contínua (a), ilustra a densidade eletrônica real, a linha tracejada (b); representa a densidade eletrônica calculada e a linha mais escura (c) representa a diferença $\left(\rho_{o}-\rho_{c}\right)$.

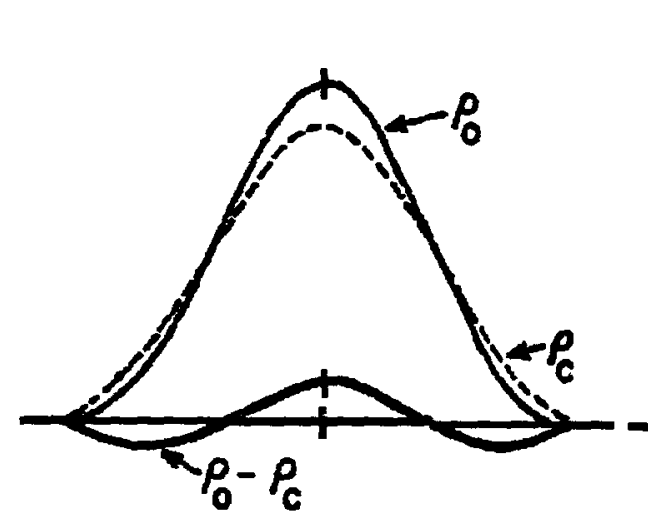

(a)

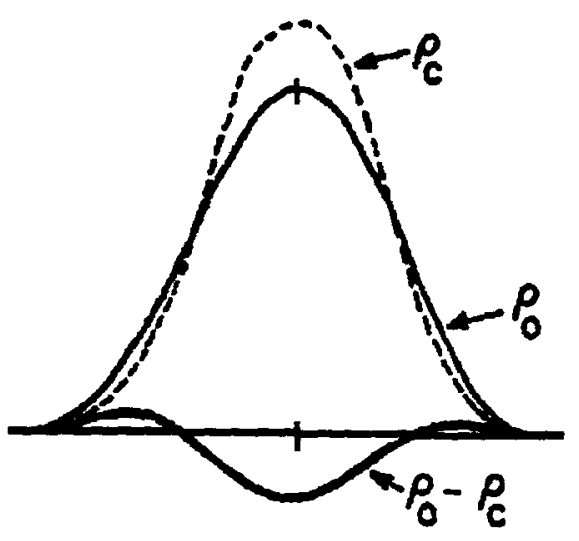

(b)

Figura 26 - Seção em linha da síntese $\rho_{0}-\rho_{c}$ : (a)- parâmetros térmicos super-estimados; (b)-parâmetros térmicos sub-estimados

Pela figura 26a verifica-se que se o parâmetro de vibração témica assumido for maior que o real, $\Delta \mathrm{F}$ mostrará um maximo positivo, e na figura $26 \mathrm{~b}$ se o parâmetro for assumido menor que o real, $\Delta \mathrm{F}$ fornecerá uma região negativa. Pode-se também mostrar que a magnitude e o sinal da curvatura do máximo ou mínimo no mapa $\Delta \mathrm{F}$ é uma medida de erros nos parâmetros.

Sabendo-se que os movimentos térmicos dos átomos não são esfericamente simétricos, numa situação anisotrópica, o movimento vibratório conduzirá a uma distribuição elipsoidal da densidade eletrônica. Isto esta ilustrado na figura 27 , que apresenta uma seção através de um átomo vibrando anisotropicamente (a), o mesmo átomo vibrando isotropicamente (b) e a diferença (c )entre (a) e (b). 


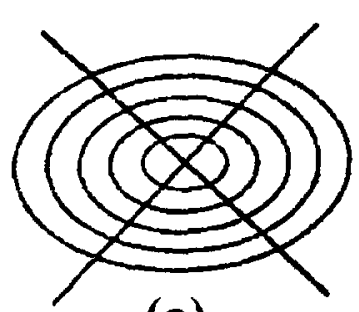

(a)

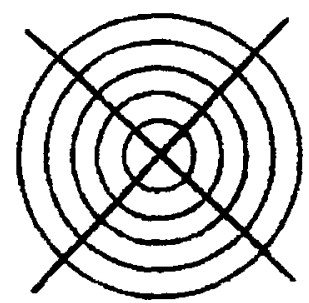

(b)

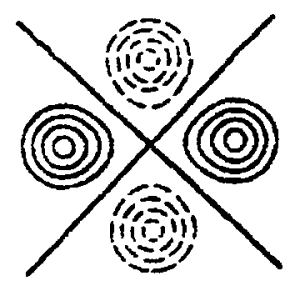

(c)

Figura 27 - Seção da densidade eletrônica por um átomo. (a) densidade eletrônica ideal, usando $F_{0}$; (b) densidade eletronica usando vibração isotrópica $\left(F_{c}\right)$; (c) densidade eletrônica $\Delta F$, mostrando a aparencia da diferença que ocorre quando um átomo vibrando anisotrópicamente é considerado isotrópico. As linhas contínuas representam regiōes positivas e as tracejadas regiões de densidade eletrônica negativa.

\subsection{2 - REFINAMENTO POR MÍNIMOS QUADRADOS}

Um método analítico de refinamento de grande poder e alcance, está baseado sobre o princípio dos mínimos quadrados [Stout e Jensen, 1989].

Considerando uma função linear com $n$ variáveis $\mathrm{x}_{1}, \mathrm{x}_{2}, \ldots, \mathrm{x}_{\mathrm{n}}$, estas variáveis, podem ser obtidas definindo um espaço, cujo valor em algum ponto é determinado pela localização $\left(x_{1}, x_{2}, x_{3}, \ldots, x_{n}\right)$ e pelos parâmetros independentes $\left(p_{1}, p_{2}, \ldots p_{n}\right)$, que definem a função,

Assim pode-se escrever a função como:

$$
f=p_{1} x_{1}+p_{2} x_{2}+p_{3} x_{3}+\ldots+p_{n} x_{n}
$$

Se os valores da função são medidos em $\mathbf{m}$ diferentes pontos com $\mathbf{m}>\mathbf{n}, 0$ princípio dos mínimos quadrados impõe que os melhores valores para os parâmetros $\mathrm{p}_{1}, \mathrm{p}_{2}, \ldots \ldots . \mathrm{p}_{\mathrm{n}}$ são aqueles que minimizam a soma dos quadrados das diferenças (propriamente pesadas) entre os valores observados e calculados da função, para todos os pontos observados. Desta forma a quantidade a ser minimizada é dada por: 


$$
D=\sum_{r=1}^{m} w_{r}\left(f_{o, r}-f_{c, r}\right)^{2}
$$

onde $\mathrm{W}_{\mathrm{r}}$ é o peso determinado de uma observação, $\mathrm{f}_{\mathrm{o} . \mathrm{r}}$ é um dos $\mathrm{m}$ valores observados da função e $f_{c, r}$ é o valor correspondente calculado.

Para obter-se as melhores correspondências será necessário, considerar os parâmetros $\mathrm{p}$ como variáveis que podem ser ajustadas para minimizar $\mathrm{D}$. Este é um problema de minimização direta que é tratado pela diferenciação do lado direito da equação (200), com respeito a cada parâmetro, e igualando a zero. Então:

$$
\sum_{r=1}^{m} w_{r}\left(f_{0, r}-f_{c, r}\right)^{2} \frac{\sigma_{c r}}{\partial p_{j}}=0, \quad \text { onde } j=1,2,3, \ldots \ldots \ldots \ldots \ldots, n
$$

que se constitui em um conjunto de $\mathrm{n}$ equações a $\mathrm{n}$ variaveis, conjunto este conhecido como equações normais.

O que ocorre na prática, é que existem m equações observadas com a forma da equação 201, sendo uma para cada observação $\left(\mathrm{F}_{\mathrm{o}}\right)$. Como o que se quer é tratar os parâmetros $p_{j}$, como quantidades a serem ajustadas, e os diferentes valores de $\mathrm{x}$ possuem diferentes valores fixados para cada uma das m observações, a situação é costumariamente reverter a ordem.

Tomando-se as derivadas parciais $\partial \mathrm{f}_{\mathrm{c} . \mathrm{r}} / \partial \mathrm{p}_{\mathrm{j}}$ para cada uma das $\mathbf{m}$ equações observadas e substituindo na equação 202 obtem-se as n equações normais representadas abaixo:

$$
\begin{aligned}
& \sum_{r=1}^{m} w_{r}\left(f_{o, r}-x_{r} p_{1}-x_{r 2} p_{2}-x_{m} p_{n}\right) x_{r 1}=0 \\
& \sum_{r=1}^{m} w_{r}\left(f_{0, r}-x_{r 1} p_{1}-x_{r 2} p_{2}-\ldots x_{m} p_{n}\right) x_{r 2}=0 \\
& \sum_{r=1}^{m} w_{r}\left(f_{0, I}-x_{r 1} p_{1}-x_{r 2} p_{2}-\ldots-x_{m} p_{n}\right) x_{m}=0
\end{aligned}
$$


Rearranjando as expressões anteriores pode-se reescrever de forma mais

completa:

$$
\begin{aligned}
& \sum_{r=1}^{m} w_{r} x_{r 1}^{2} p_{1}+\sum_{r=1}^{m} w_{r} x_{r 1} x_{r 2} p_{2}+\ldots+\sum_{r=1}^{m} w_{r} x_{r 1} x_{m} p_{n}=\sum_{r=1}^{m} w_{r} f_{0, r} x_{r 1} \\
& \sum_{r=1}^{m} w_{r} x_{r 2} x_{r 1} p_{1}+\sum_{r=1}^{m} w_{r} x_{r 2}^{2} p_{2}+\ldots+\sum_{r=1}^{m} w_{r} x_{r 2} x_{m} p_{n}=\sum_{r=1}^{m} w_{r} f_{o, r} x_{r 2} \\
& \sum_{r=1}^{m} w_{r} x_{r n} x_{r 1} p_{1}+\sum_{r=1}^{m} w_{r} x_{r} x_{r 2} p_{2}+\ldots+\sum_{r=1}^{m} w_{r} x_{r n}^{2} p_{n}=\sum_{r=1}^{m} w_{r} f_{o, r} x_{m}
\end{aligned}
$$

A solução deste sistema de $\mathbf{n}$ equações fornece diretamente os melhores valores dos parâmetros $p_{\mathrm{j}}$ no sentido dos mínimos quadrados.

Se a forma funcional das equações observadas não é linear nos valores de $p$, então as equações normais não são lineares tornando-se insolúveis, entretanto nestes casos , pode ser tornadas lineares por uma aproximação da função em série de Taylor:

$$
f\left(p_{1}, p_{2}, \ldots, p_{n}\right)=f\left(a_{1}, a_{2} \ldots, a_{n}\right)+\frac{\partial\left(a_{1}, a_{2}, \ldots a_{n}\right)}{\partial p_{1}}\left(p_{1}-a_{1}\right)+\ldots \frac{\partial\left(a_{1}, a_{2}, \ldots a_{n}\right)}{\partial p_{n}}\left(p_{n}-a_{n}\right)
$$

ou

$$
f\left(p_{1}, p_{2}, \ldots, p_{n}\right)=f\left(a_{1}, a_{2} \ldots, a_{n}\right)+\frac{\partial\left(a_{1}, a_{2}, \ldots a_{n}\right)}{\partial p_{1}} \Delta p_{1}+\ldots \frac{\partial\left(a_{1}, a_{2}, \ldots . a_{n}\right)}{\partial p_{n}} \Delta p_{n}
$$

onde termos de $\Delta p_{j}$, de potencia maior que um foram desprezados. Os $a_{j}$, são valores aproximados de $p_{i}$, e $f\left(a_{1}, a_{2}, \ldots, a_{n}\right) ; \partial f\left(a_{1}, a_{2}, ., a_{n}\right) / \partial p_{j} ; \partial f\left(a_{1}, a_{2}, \ldots, a_{n}\right) / \partial p_{n}$, são as funções e suas derivadas avaliadas em seus valores aproximados.

Se os valores de $a_{j}$ são aproximações suficientes boas, a aplicação dos processos de mínimos quadrados usando as equações lineares dadas pela equação 204 fornecerá valores para as quantidades $\Delta \mathrm{p}_{\mathrm{j}}$, de modo que os $\mathrm{a}_{\mathrm{i}}$ sejam dados por:

$$
\mathrm{a}_{\mathrm{i}}{ }^{\prime}=\mathrm{a}_{\mathrm{i}}+\Delta \mathrm{p}_{\mathrm{j}}
$$


Portanto uma aproximação melhor para os melhores valores dos parâmetros $\mathrm{p}_{\mathrm{j}}$, melhores então do que os iniciais $a_{j}$.

Por outro lado devido as séries terem sido truncadas pelo despreso de altas potencias de $\Delta \mathrm{p}_{\mathrm{j}}$, os cálculos devem ser repetidos usando como valores aproximados para cada repetição os resultados derivados dos cálculos precedentes.

O processo interativo está completo quando não existe variação significativa nos parâmetros entre dois ciclos sucessivos, isto está em contraste para o caso onde as equações observadas são verdadeiramente lineares, quando nenhuma aproximação nos parâmetros é requerida e a solução das equações normais fornece seus valores interativos.

Em difração de raios-X, as formas funcionais dos fatores de estrutura são transcendentais e assim devem ser aproximadas por uma série de Taylor truncada, então neste caso a quantidade minimizada é dada por:

$$
\mathrm{D}=\sum_{\mathrm{hk} /} \mathrm{w}_{\mathrm{hk} /}\left(\left|\mathrm{F}_{\mathrm{o}}\right|-\left|\mathrm{kF}_{\mathrm{c}}\right|\right)^{2}
$$

Desta forma a quantidade D é minimizada sobre todas as reflexões observadas.

A minimização é feita como no caso anterior tomando-se a derivada com respeito a cada parâmetro e igualando a zero, o que conduzirá às $n$ equações similares a equação 202:

$$
\sum_{k k l} w_{h k}\left(\left|F_{o}\right|-\left|k F_{c}\left(p_{1}, p_{2}, \ldots, p_{n}\right)\right| \frac{\partial\left|k F_{c}\left(p_{1}, p_{2}, \ldots p_{n}\right)\right|}{\partial p_{j}}=0 \quad \text { com } j=1,2,3 \ldots n\right.
$$

Expressando-se a função $\left|F_{c}\right|$ como uma série de Taylor e negligênciando os termos de potência maior que um:

$$
\left|k F_{c}\left(p_{1}, \ldots, p_{n}\right)\right|=\left|k F_{c}\left(a_{1} \ldots, a_{n}\right)\right|+\frac{\partial\left|k F_{c}\right|}{\partial_{1}} \Delta p_{1}+\ldots+\frac{\partial\left|k F_{c}\right|}{\partial_{n}} \Delta p_{n}
$$


onde $\mathrm{p}_{\mathrm{l}}, \ldots, \mathrm{p}_{\mathrm{n}}$, podem ser algum dos parâmetros: escala, posicionais ou térmicos, e. $\Delta \mathrm{p}_{\mathrm{j}}=\mathrm{p}_{\mathrm{j}}-\mathrm{a}_{\mathrm{j}}$ Substituindo a equação209 em 208 tem-se:

$$
\sum_{\mathrm{h} l} \mathrm{w}_{\mathrm{hk} l}\left(\left|\mathrm{~F}_{\mathrm{o}}\right|-\left|\mathrm{kF}_{\mathrm{c}}\left(\mathrm{a}_{1}, \ldots, \mathrm{a}_{\mathrm{n}}\right)\right|-\frac{\partial\left|\mathrm{kF} \mathrm{F}_{\mathrm{c}}\right|}{\partial \mathrm{p}_{1}} \Delta \mathrm{p}_{1}-\ldots-\frac{\partial\left|\mathrm{kF}_{\mathrm{c}}\right|}{\partial \mathrm{p}_{\mathrm{n}}} \Delta \mathrm{p}_{\mathrm{n}}\right) \frac{\partial|\mathrm{kF}|}{\partial \mathrm{p}_{\mathrm{j}}}=0
$$

onde $\mathrm{j}=1,2,3 \ldots, \mathrm{n}$

A equação 210 pode ser escrita ainda de uma outra forma:

$$
\sum_{\mathrm{h} J} \mathrm{w}_{\mathrm{hk} l}\left(\Delta \mathrm{F}-\frac{\partial\left|\mathrm{kF}_{\mathrm{c}}\right|}{\partial \mathrm{p}_{1}} \Delta \mathrm{p}_{1}-\ldots-\frac{\partial\left|k \mathrm{~F}_{\mathrm{c}}\right|}{\partial \mathrm{p}_{\mathrm{n}}} \Delta \mathrm{p}_{\mathrm{n}}\right) \frac{\partial\left|k \mathrm{kF}_{\mathrm{c}}\right|}{\partial \mathrm{p}_{\mathrm{j}}}=0
$$

onde $\Delta F$ tem o papel das quantidades observadas $f_{o}$ nas equações 201 e 202 respectivamente.

Expandindo-se e rearranjando a equação 211 obtem-se um conjunto de equações, que pode ser comparado termo a termo com a equação 203.

$$
\begin{aligned}
& \sum_{r=1}^{m} w_{r}\left(\frac{\partial\left|k F_{c, r}\right|}{\partial p_{1}}\right)^{2} \Delta p_{1}+\sum_{r=1}^{m} w_{r} \frac{\partial\left|k F_{c . r}\right|}{\partial p_{1}} \frac{\partial\left|k F_{c . r}\right|}{\partial p_{2}} \Delta p_{2}+\ldots+\sum_{r=1}^{m} w_{r} \frac{\partial\left|k F_{c . r}\right|}{\partial p_{1}} \frac{\partial\left|k F_{c, r}\right|}{\partial p_{n}} \Delta p_{n}=\sum_{r=1}^{m} w_{r} \Delta F_{r} \frac{\partial\left|k F_{c . r}\right|}{\partial p_{1}} \\
& \sum_{\mathrm{r}=1}^{m} \mathrm{w}_{\mathrm{r}} \frac{\partial\left|k \mathrm{~F}_{\mathrm{c}, \mathrm{r}}\right|}{\partial \mathrm{p}_{2}} \frac{\partial\left|k \mathrm{~F}_{\mathrm{c}, \mathrm{r}}\right|}{\partial \mathrm{p}_{1}} \Delta \mathrm{p}_{1}+\sum_{\mathrm{r}=1}^{\mathrm{m}} \mathrm{w}_{\mathrm{r}}\left(\frac{\partial\left|k \mathrm{~F}_{\mathrm{c}, \mathrm{r}}\right|}{\partial \mathrm{p}_{2}}\right)^{2} \Delta \mathrm{p}_{2}+\ldots+\sum_{\mathrm{r}=1}^{\mathrm{m}} \mathrm{w}_{\mathrm{r}} \frac{\partial\left|k \mathrm{~F}_{\mathrm{c}, \mathrm{r}}\right|}{\partial \mathrm{p}_{2}} \frac{\partial\left|k \mathrm{~F}_{\mathrm{c}, \mathrm{r}}\right|}{\partial \mathrm{p}_{\mathrm{n}}} \Delta \mathrm{p}_{\mathrm{n}}=\sum_{\mathrm{r}=1}^{\mathrm{m}} \mathrm{w}_{\mathrm{r}} \Delta \mathrm{F}_{\mathrm{r}} \frac{\partial\left|k \mathrm{~F}_{\mathrm{c}, \mathrm{r}}\right|}{\partial \mathrm{p}_{2}}
\end{aligned}
$$

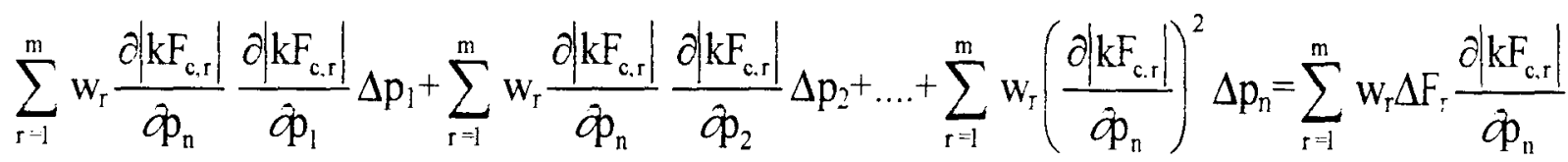

Este é um sistema de $n$ equações sobre $n$ incognitas $\Delta p_{j}$. Observa-se que estas equações são lineares sobre os $\Delta \mathrm{p}_{\mathrm{i}}$ e tem solução. A combinação destas equações com as aproximações iniciais dos $a_{i}$ fornece os melhores valores aproximados para vários parâmetros, podendo ser usada para a repetição do processo, assim as convergências são obtidas até que os sucessivos ciclos não produzam variações significativas. 


\subsection{3 - FUNCÕES PESO}

As funções minimizadas pelos métodos de mínimos quadrados trazem consigo, o fator de pesagem (w), como sendo uma medida de segurança das observações. Escolhida adequadamente, possui o efeito de ajustar a contribuição de cada observação para as equações normais com o objetivo de produzir melhores resultados.

Existem diversos esquemas de pesagem, cada um incluindo diferentes considerações. Para a maioria dos sistemas de peso, as reflexões não observadas são omitidas das equações de minimização, portanto tem peso zero.

A seguir faz-se uma breve descrição dos principais esquemas de peso.

\subsubsection{CONTRIBUIÇÃO DE NON POISSON}

Este esquema de peso é dado pela equação abaixo notando que quando a reflexão obedece $\mathrm{F}^{2} \leq$ corte $\bullet \sigma\left(\mathrm{F}^{2}\right)$, ou seja é não observada, então a reflexão será omitida:

$$
w=\frac{1}{\sigma(\mathrm{F})^{2}}
$$

onde,

$$
\begin{aligned}
\sigma(\mathrm{F}) & =\frac{\sigma\left(\mathrm{F}^{2}\right)}{2 \mathrm{~F}} \\
\sigma\left(\mathrm{F}^{2}\right) & =\left(\sigma(\mathrm{l})^{2}+\left(\mathrm{p} \cdot \mathrm{F}^{2}\right)^{2}\right)^{1 / 2}
\end{aligned}
$$

Para estas equações tem-se:

F: é o fator de estrutura com desvio padrão $\sigma(F)$;

$\sigma(\mathrm{I})$ : é o desvio padrão de I, baseado sobre a contagem estatística;

w: peso para a reflexão;

p: fator de instabilidade experimental usado para diminuir o efeito de reflexões mais fortes. Este fator depende sobre a estabilidade dos dados coletados experimentalmente, sendo valores entre 0,02 e 0,07 são geralmente apropriados. No sistema MolEN [Enraf-Nonius, 1990), o valor é tomado como 0,04 


\subsubsection{2 - PESO PIVOT-POINT}

$O$ "pivot" é definido como sendo $1 / 3$ do valor de $F_{\max }$, sendo $F_{\max }$ o valor máximo do fator de estrutura observado.

Para o sistema de peso pivot-point são definidas as seguintes situações:

Se $F<$ pivô, então $w=F /$ pivot;

Se $\mathrm{F} \geq$ pivô, então $\mathrm{w}=$ pivot $/ \mathrm{F}$;

Se $F<$ corte então $w=0$.

\subsubsection{3 - PESO UNITÁRIO}

Neste caso o valor de w será assumido igual a 1, para todas as reflexões, com exceção das não observadas, para as quais $\mathrm{w}=0$, ou seja, se $\mathrm{I} \leq$ corte $\cdot \sigma(\mathrm{I})$ onde o corte é um parâmetro definido.

\subsubsection{4 - PESO UNITÁRIO MODIFICADO}

Neste sistema de pesagem de reflexões, o fator de estrutura apresenta duas situações definidas pela figura28.

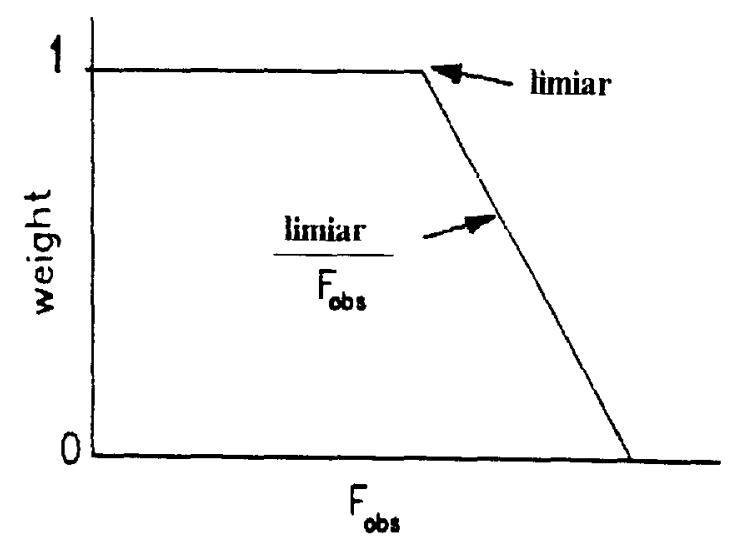

Figura 28 - Gráfico do limiar para o sistema de peso unitário modificado.

Usando-se o gráfico se $\mathrm{F}>$ limiar então: $\mathrm{W}=1,0$; se $\mathrm{F} \leq$ limiar a equação para 0 peso será dada por :

$$
\mathrm{w}=[\operatorname{limiar} / \mathrm{F}]^{2}
$$

Se $F$ for nâo observado, $W=0$. 


\subsubsection{5 - PESO POLINOMIAL CRUICKSHANK}

Este peso é dado pela equação:

$$
W=\frac{1}{C_{0}+C_{1} F+C_{2} F^{2}+C_{3} F^{3}}
$$

Onde os coeficientes $\mathrm{C}_{0}, \mathrm{C}_{1}, \mathrm{C}_{2} \mathrm{e} \mathrm{C}_{3}$ são normalmente definidos pelo usuário.

\subsubsection{6 - PESO POLINOMIAL MODIFICADO DE CRUICKSHANK}

Este tipo de peso inclui os coeficientes largura e altura da reflexão, e é usualmente definido por:

$$
\mathrm{w}=\frac{1}{1+\left[\frac{\mathrm{F}-\text { altura }}{\text { largura }}\right]^{2}}
$$

Os coeficientes altura e largura são definidos pelo usuário.

\subsubsection{7 - MÉTODO DE PESAGEM KILLEAN E LAWRENCE [1969]}

A expressão para este peso é dada por :

$$
w=\frac{1}{\sigma F^{2}+(P W T \cdot F)^{2}+Q W T}
$$

onde PWT e QWT são valores usualmente definidos com valores 0.02 e 1 respectivamente. Valores típicos para PWT são de 0.02 a 0.03 e para QWT são de 0 a 3 dependendo dos dados de intensidade. O fator PWT ajusta a contribuição dos F mais altos, enquanto que QWT tem maior eficiência sobre os valores de $\mathrm{F}$ pequenos. 


\subsection{4. - CONTROLE DE REFINAMENTO E INDICE DE DISCORDÂNCIA}

Um índice de verificação da adequação do sistema de pesos utilizado no programa de refinamento por métodos de mínimos quadrados é denominado 'godness of fit' (GOF ou S) dado pela equação:

$$
\mathrm{GOF}=\sqrt{\frac{\sum_{i=1}^{\mathrm{m}} \mathrm{w}\left\|\mathrm{Fo}_{\mathrm{i}}|-| \mathrm{Fc}_{\mathrm{i}}\right\|^{2}}{\text { graus de liberdade }}}
$$

onde graus de liberdade corresponde ao número de reflexões menos o número de variáveis.

A equação 219 , algumas vezes também chamada de desvio padrão de uma observação de pesos unitários (S), e é uma medida dos graus para os quais a distribuição encontrada das diferenças entre $\left|F_{o}\right|$ e $\left|F_{c}\right|$ adequa as distribuições esperadas dos pesos usados no refinamento.

Os controles de refinamento de uma estrutura mais usuais são os chamados índices de discordância, $\mathrm{R}$.

Dentre os mais utilizados colocam-se:

$R$ não pesado (unweighted $R$ value), dado pela expressão:

$$
R=\sqrt{\frac{\sum_{i=1}^{m} \| \mathrm{Fo}_{i}|-| \mathrm{Fc}_{i}||^{2}}{\sum_{i-1}^{\mathrm{m}}\left|\mathrm{Fo}_{i}\right|^{2}}}
$$

$R$ pesado (weighted $R$ ), dado pela equação:

$$
R=\sqrt{\frac{\sum_{i-1}^{m} w|| F o_{i}|-| F c_{i} \|^{2}}{\sum_{i=1}^{m} w\left|F o_{i}\right|^{2}}}
$$

Rall, ou R sobre todas as reflexões é calculado como $R$ pesado ( com peso unitário), incluindo todas as reflexões, é o único que inclue as não observadas. 


\section{CAPÍTULO II}

\section{DETERMINAÇ̃̃O DA ESTRUTURA CRISTALINA E MOLECULAR DO \\ 5,4'- DIHIDROXI - 3',5'- DIMETOXI - 6,7 - (2", 2", DIMETILPIRANO) FLAVONA}

\section{1 - INTRODUÇÃO}

Como motivação deste trabalho e dos pesquisadores que atuam nesta área da Ciência, pode-se parafrasear o Prof. Dr. Nilso Barelli:

"Os motivos que levam a resolver as estruturas das substâncias cristalinas, naturais ou artificiais, se aprofundam na indagação da própria natureza, fascínio pela estrutura do mundo em que vivemos, o que na verdade constitui uma atitude profundamente religiosa."

Neste capítulo será apresentada a estrutura cristalina e molecular do composto 5,4',-Dihidroxi-3',5'- dimetoxi- 6,7-(2",2"'- dimetilpirano)flavona.

Os monocristais foram fornecidos pelo Prof. Dr. Lourivaldo da Silva Santos da Universidade Federal do Pará, com o objetivo da determinação de sua estrutura molecular, para efeito de comparação com resultados espectroscópicos.

Para obter melhores monocristais a amostra foi recristalizada utilizando como solvente álcool etilico e deixada a baixa temperatura (geladeira). 


\subsection{COLETA DE DADOS}

Um monocristal do composto de cor amarela transparente, de dimensões 0,10 $\times 0,08 \times 0,12 \mathrm{~mm}$, foi selecionado e colado em uma fibra de vidro, sendo então montado no difratômetro $\mathrm{CAD}-4$ da Enraf-Nonius para a realização das medidas dos parâmetros da cela unitária e das intensidades das reflexões.

Os ângulos de posicionamento para 25 reflexões, localizadas e centradas automaticamente, permitiram calcular e refinar os parâmetros da cela unitária.

No experimento as medidas de intensidades foram feitas utilizando-se a radiação $\mathrm{K}_{\alpha}(\mathrm{Mo})(\lambda=0.71073 \AA)$, monocromatizada por um cristal de grafite no modo ômega $(\omega)$, com um intervalo de $2 \theta$ entre 0 e $41.2^{\circ}$, e velocidade de varredura máxima igual a $16,48^{\circ} / \mathrm{min}$, e minima de $1,83^{\circ} / \mathrm{min}$.

Durante a coleta de dados foram usadas três reflexões $(\overline{1}, 11, \overline{2} ; 0 \overline{6} \overline{4} ; 6,0, \overline{12})$, para o controle das intensidades, as quais foram registradas a cada $2 \mathrm{~h}$, observando-se que a variação foi menor que 5\%.

Tendo-se em vista o limite máximo de $\theta$ mediu-se as reflexões com índices no intervalo $-13 \leq \mathrm{h} \leq 13 ; 0 \leq \mathrm{k} \leq 23 ;-13 \leq l \leq 0$; sendo coletadas 4118 reflexões, das quais 3952 reflexões independentes, destas, 1743 , foram consideradas observadas com $I \geq 3 \sigma(I)$.

O cristal de 5,4',-Dihidroxi- 3',5'- dimetoxi- 6,7-(2",2"- dimetilpirano)flavona pertence ao sistema cristalino monoclínico, com: $a=13,561(1), b=23,428(2), c=13,725(1) \AA$, $\beta=119,528(4)^{0}, V=3819,6(5) \AA^{3}$

A análise das extinções sistemáticas $(\mathrm{h} k l$ com $\mathrm{h}+\mathrm{k}=2 \mathrm{n})$ levou a uma cela unitária centrada $\mathrm{C}$; a presença de reflexões hol somente do tipo $l=2 \mathrm{n}$ indicou duas possibilidades de grupo espacial: $\mathrm{C} 2 / \mathrm{c}$ ou Cc. 
$O$ cálculo da densidade supondo $Z=8$ moleculas/cela unitária resultou no valor $D_{c}=1,366 \mathrm{~g} \cdot \mathrm{cm}^{-3}$, coerente com o esperado para este tipo de composto (calculando para $\mathrm{Z}=4$, obtem-se $\mathrm{D}_{\mathrm{c}}=0.683 \mathrm{~g} \cdot \mathrm{cm}^{-3}$ ).

A escolha do grupo espacial $\mathrm{C} 2 / \mathrm{c}$ foi confirmada como correta quando do cálculo da distribuição estatística dos fatores de estrutura, que apresentaram distribuição correspondente a cristal centrossimétrico $(<|\mathrm{E}|>=0,670)$.

\section{Tabela 3}

\section{RESUMO DOS PRINCIPAIS DADOS CRISTALOGRÁFICOS}

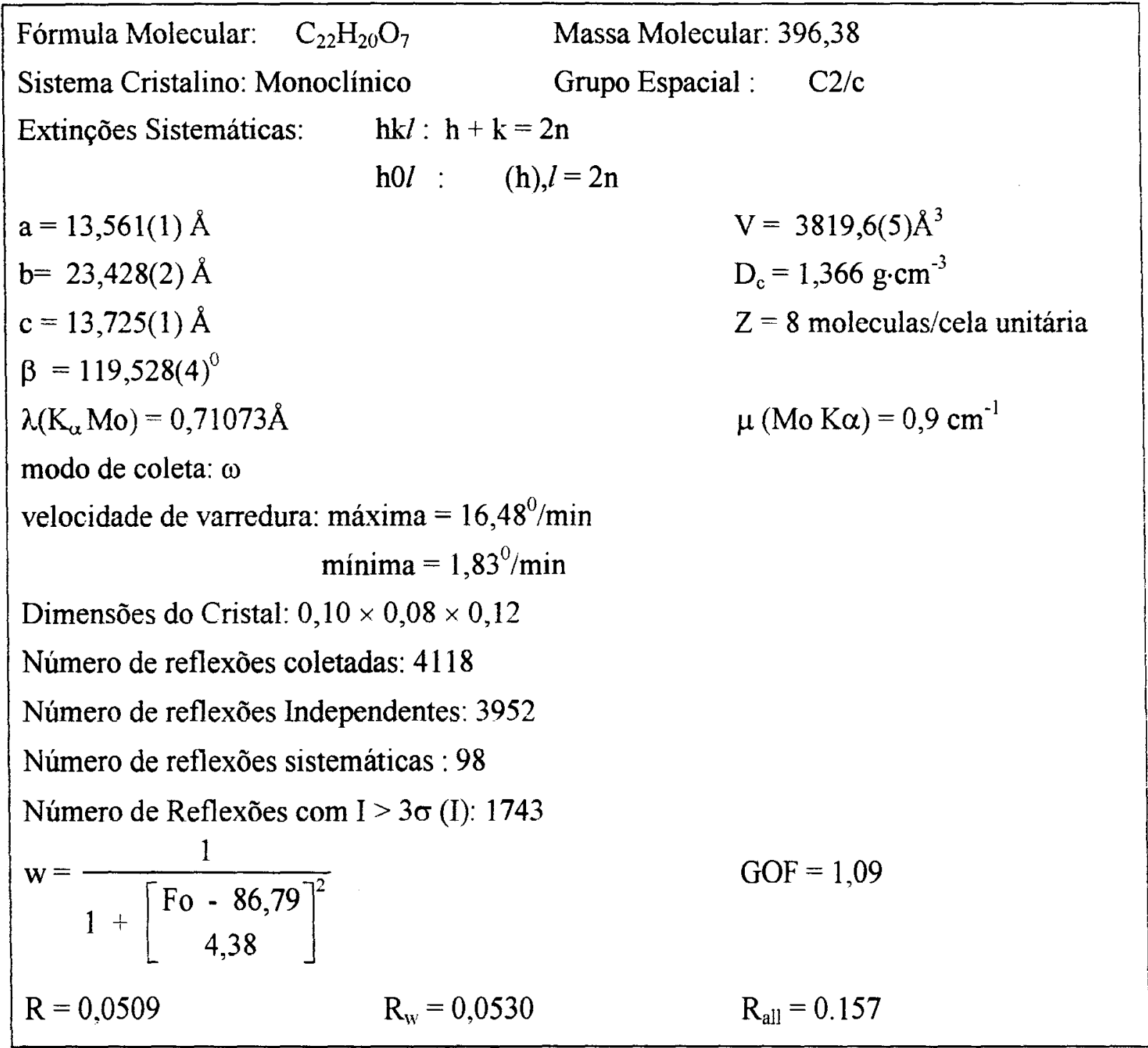




\section{3 - SOLUÇÃO E REFINAMENTO DA ESTRUTURA}

Os dados de intensidades foram reduzidos a módulos dos fatores de estrutura com seus respectivos desvios padrão, após a correção pelos fatores de Lorentz polarização e absorção pelo método empírico PSISCAN [Enraf-Nonius, 1990].

Os índices de consistência interna calculados para os dados foram: 0,016 e 0,021 , para as reflexões observadas e todas respectivamente, quando considerou-se as intensidades; e 0,015 e 0,055 considerando-se os fatores de estrutura.

Os fatores de transmissão máximos e mínimo foram respectivamente: $0,9998 \mathrm{e}$ 0,9648, respectivamente, e o médio igual a 0,9791 .

A estrutura foi resolvida utilizando Métodos Diretos pelo programa SIR [Enraf- Nonius, 1990], tendo sido localizados todos os átomos, exceto os hidrogênios.

Os fatores de escala e temperatura isotrópico médio foram conseguidos pelo gráfico de Wilson com os seguintes valores: $\mathrm{K}=10.74637$ e $\mathrm{B}=3.83207$.

Inicialmente as relações de fase foram obtidas, após a normalização dos fatores de estrutura, utilizando-se as reflexões com $\mathrm{E} \geq 1.836$, gerando 3229 tripletes positivos, 173 negativos e 500 quartetos negativos.

A fixação da origem da cela unitária foi efetuada pelas reflexões 715 e $8,18, \overline{7}$, e os símbolos foram selecionados para as seguintes reflexões: $570 ; 5,1,10 ; 6,0, \overline{12} ; 408$ e $4,16,1$.

A partir do conjunto que apresentou as melhores figuras de mérito, calculou-se um mapa de E que mostrou máximos de densidade eletrônica, todos com aproximadamente a mesma intensidade, para os átomos de carbono e oxigênio. 
O refinamento da estrutura foi feito pelo método de mínimos quadrados utilizando o sistema de programa Molen [Enraf-Nonius, 1990], instalado no computador VAX 4600 do Instituto de Química de São Carlos USP.

Foram usados os fatores de espalhamento atômico de Cromer e Waber [1974] para os átomos não hidrogênio, e os de Stewart e outros [1965] para os átomos de hidrogênio.

Os parâmetros posicionais e de vibração térmica isotrópica foram refinados por 3 ciclos,considerando-se todos os átomos como carbono, resultando em um fator de discordância $\mathrm{R}=0,36$, usando peso unitário. Após o cálculo do mapa de Fourier diferença verificou-se que os átomos estavam corretamente localizados, e foi possivel distinguir os oxigênios.

Manteve-se os átomos refinando com fator de vibração térmica isotrópico até $\mathrm{R}=0,19$, e após transforma-los em anisotrópicos, o índice de discordância tornou-se igual a $\mathrm{R}=0,079$.

Finalmente os átomos de hidrogênio foram posicionados, de acôrdo com a geometria do átomo ao qual estavam ligados, quando $\mathrm{R}=0,072$. Suas posições não foram refinadas , mas sim recalculadas a cada estágio de refinamento, até a convergência final. Foi adotado o valor de $6,0 \AA^{2}$ para o fator de temperatura dos hidrogênios.

Os hidrogênios das hidroxilas (O5-H e O7-H) foram localizados nos mapas de Fourier diferença, mas não foram refinados.

Os parâmetros atômicos, exceto os hidrogênios, foram refinados por mínimos quadrados usando a matriz completa até $\mathrm{R}=0,0509$ e $\mathrm{R}_{\mathrm{w}}=0,0530$, com peso Cruickshank modificado (equação 216), com largura igual 4,38 e altura igual a 86,79. Neste estágio GOF = 1,09 e $R_{\mathrm{all}}=0.157$

Durante os estágios finais do refinamento foi incluido o fator de extinção, e refinado, seu valor final foi de $1,24(8) \cdot 10^{-7}$. 
Foram refinados 263 parâmetros, com 1763 reflexões observadas.

Durante todo o processo de refinamento foram efetuados cálculos de distâncias e ângulos interatômicos, para verificação da coerência química do modelo refinado. Os programas utilizados foram ESD e BOND do sistema MolEN [Enraf- Nonius, 1990].

No estágio final do refinamento o mapa de Fourier diferença mostrou densidade eletrônica positiva máxima igual a $0,27(2)$ e $/ \AA^{3}$ e negativa igual a -012(2) e/ $\AA^{3}$.

\section{4 - RESULTADOS E CONCLUSÕES}

A estrutura molecular apresentou-se como proposta pelo Prof. Dr. Lourivaldo da S. Santos.

A estrutura cristalina consiste de uma molécula por unidade assimétrica, com a formação de ligações de hidrogênio intra e intermoleculares.

Uma representação ORTEP [Jonhson, 1965] da estrutura molecular do composto, está na figura 29 com os átomos identificados.

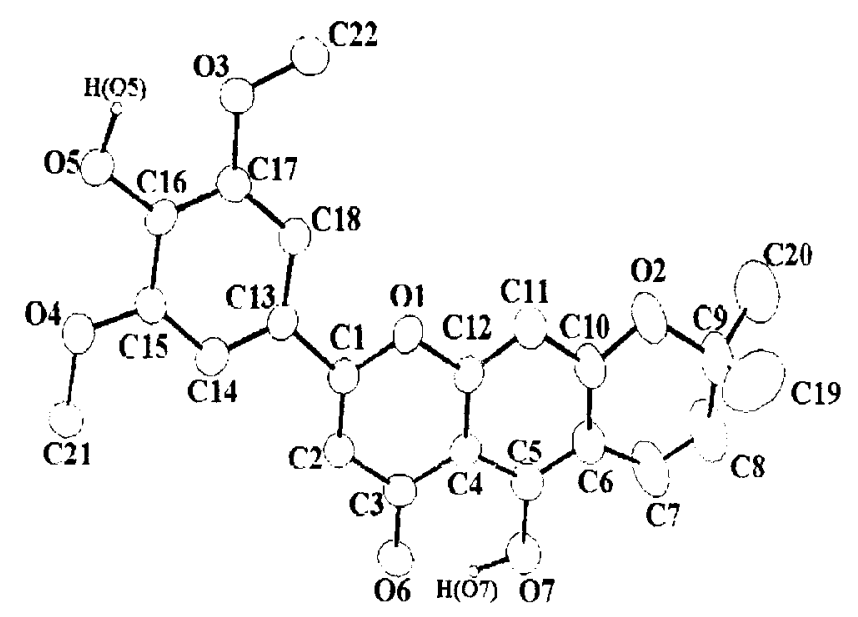

Figura 29 - Representação Ortep da molécula de 5,4'- dihidroxi - 3',5'- dimetoxi - 6,7 (2", 2", dimetilpirano) flavona 
$\mathrm{Na}$ figura 30 é feita uma representação ORTEP com os elipsóides com $50 \%$ de probabilidade.

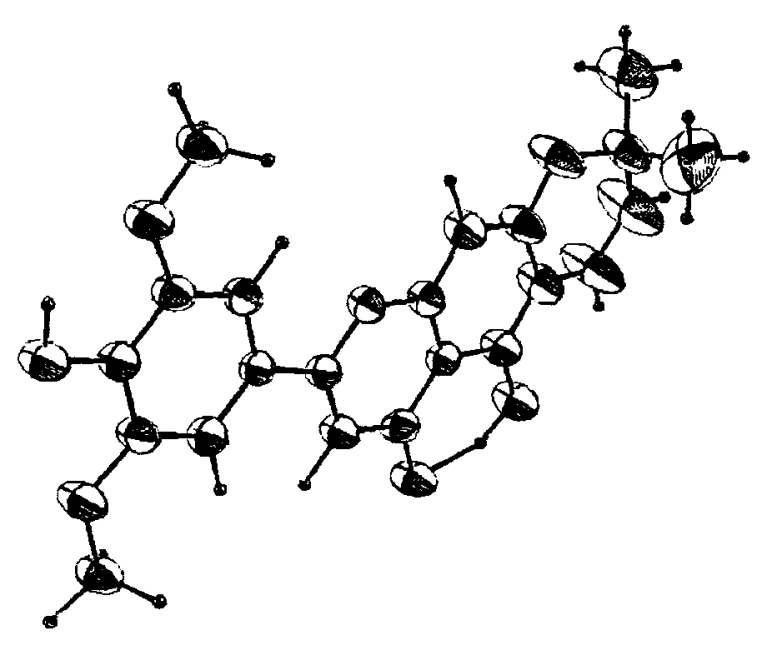

Figura 30 - Representação Ortep da Molécula de 5,4'- Dihidroxi - 3',5'- dimetoxi - 6,7 (2", 2", dimetilpirano) flavona com os elipsoides de vibração térmica.

Os parâmetros posicionais com os respectivos fatores de vibração térmica isotrópica equivalentes de todos os átomos, exceto os hidrogênios, estão listados na tabela 4 . Os parâmetros posicionais dos átomos de hidrogênios estão na tabela 5.

A tabela 6 apresenta os parâmetros de vibração térmica anisotrópica dos átomos, exceto para os átomos de hidrogênios.

Na tabela 7 encontram-se as distâncias interatômicas com seus desvios padrão.

A tabela 8 mostra os ângulos interatômicos com seus desvios padrão.

Os fatores de estrutura calculados e observados finais estão listados no Apendice 1.

A tabela 9 contém os ângulos de torção calculados para a molécula de 5,4'dihidroxi-3',5'-dimetoxi-6,7-(2', 2''-dimetilpirano) flavona. 


\section{TABELA 4}

COORDENADAS ATÔMICAS FRACIONÁRIAS E FATORES DE VIBRAÇÃO TÉRMICA ISOTRÓPICOS EQUIVALENTES $\left(\AA^{2}\right)$ COM OS RESPECTIVOS DESVIOS PADRÃO ENTRE PARÊNTESES EXCLUIDOS OS HIDROGENIOS PARA O 5,4'-DIHIDROXI-3',5'-DIMETOXI-6,7-(2"',2"'-DIMETILPIRANO) FLAVONA

\begin{tabular}{|c|c|c|c|c|}
\hline Átomo & $\mathbf{x}$ & $\mathbf{y}$ & $\mathbf{z}$ & Beq \\
\hline 01 & $0.9828(2)$ & $0.5515(1)$ & $0.1211(2)$ & $3.38(7)$ \\
\hline $\mathrm{O} 2$ & $1.1064(2)$ & $0.7399(1)$ & $0.1908(3)$ & $6.5(1)$ \\
\hline 03 & $0.5870(2)$ & $0.4812(1)$ & $-0.0850(3)$ & $4.73(8)$ \\
\hline $\mathrm{O} 4$ & $0.7937(2)$ & $0.3136(1)$ & $0.0148(3)$ & $5.25(9)$ \\
\hline O5 & $0.6014(2)$ & $0.3674(1)$ & $-0.0846(3)$ & $5.13(9)$ \\
\hline O6 & $1.3018(2)$ & $0.4883(1)$ & $0.2675(3)$ & $4.77(8)$ \\
\hline 07 & $1.3781(2)$ & $0.5903(1)$ & $0.3116(3)$ & $5.07(9)$ \\
\hline $\mathrm{Cl}$ & $1.0014(3)$ & $0.4943(2)$ & $0.1256(3)$ & $2.93(9)$ \\
\hline $\mathrm{C} 2$ & $1.1059(3)$ & $0.4723(2)$ & $0.1736(3)$ & $3.4(1)$ \\
\hline $\mathrm{C} 3$ & $1.2038(3)$ & $0.5084(2)$ & $0.2229(3)$ & $3.3(1)$ \\
\hline $\mathrm{C} 4$ & $1.1832(3)$ & $0.5684(2)$ & $0.2189(3)$ & $3.03(9)$ \\
\hline $\mathrm{C} 5$ & $1.2702(3)$ & $0.6093(2)$ & $0.2632(4)$ & $3.9(1)$ \\
\hline C6 & $1.2482(3)$ & $0.6668(2)$ & $0.2569(4)$ & $4.7(1)$ \\
\hline $\mathrm{C} 7$ & $1.3333(3)$ & $0.7111(2)$ & $0.2935(6)$ & $8.5(2)$ \\
\hline $\mathrm{C} 8$ & $1.3042(4)$ & $0.7649(2)$ & $0.2914(6)$ & $9.6(2)$ \\
\hline C9 & $1.1891(3)$ & $0.7831(2)$ & $0.2595(4)$ & $5.5(1)$ \\
\hline $\mathrm{C} 10$ & $1.1351(3)$ & $0.6840(2)$ & $0.2050(4)$ & $4.4(1)$ \\
\hline $\mathrm{C} 11$ & $1.0469(3)$ & $0.6458(2)$ & $0.1583(4)$ & $4.0(1)$ \\
\hline $\mathrm{C} 12$ & $1.0725(3)$ & $0.5887(2)$ & $0.1672(3)$ & $3.2(1)$ \\
\hline $\mathrm{C} 13$ & $0.8949(3)$ & $0.4618(2)$ & $0.0716(3)$ & $2.92(9)$ \\
\hline $\mathrm{Cl} 4$ & $0.8987(3)$ & $0.4027(2)$ & $0.0717(3)$ & $3.5(1)$ \\
\hline$C 15$ & $0.8000(3)$ & $0.3714(2)$ & $0.0198(4)$ & $3.7(1)$ \\
\hline $\mathrm{C} 16$ & $0.6965(3)$ & $0.3999(2)$ & $-0.0335(4)$ & $3.7(1)$ \\
\hline $\mathrm{C} 17$ & $0.6936(3)$ & $0.4586(2)$ & $-0.0315(4)$ & $3.6(1)$ \\
\hline $\mathrm{C} 18$ & $0.7920(3)$ & $0.4906(2)$ & $0.0197(3)$ & $3.3(1)$ \\
\hline $\mathrm{C} 19$ & $1.1759(5)$ & $0.7914(3)$ & $0.3609(6)$ & $9.8(3)$ \\
\hline $\mathrm{C} 20$ & $1.1530(4)$ & $0.8357(2)$ & $0.1893(5)$ & $7.8(2)$ \\
\hline $\mathrm{C} 21$ & $0.8967(3)$ & $0.2824(2)$ & $0.0682(4)$ & $4.8(1)$ \\
\hline $\mathrm{C} 22$ & $0.5760(3)$ & $0.5414(2)$ & $-0.0874(4)$ & $5.3(1)$ \\
\hline
\end{tabular}

TABELA 5

COORDENADAS ATÔMICAS FRACIONÁRIAS DOS ÁTOMOS DE HIDROGÊNIO PARA 0 5,4'-DIHIDROXI-3',5'-DIMETOXI-6,7-(2",2"'-DIMETILPIRANO)

FLAVONA.

\begin{tabular}{|lccl|llcc|}
\hline Átomo & $\mathbf{x}$ & $\mathbf{y}$ & $\mathbf{z}$ & Átomo & $\mathbf{x}$ & $\mathbf{y}$ & $\mathbf{z}$ \\
$\mathrm{H}(\mathrm{O} 5)$ & 0.5375 & 0.3950 & -0.1049 & $\mathrm{H} 193$ & 1.2341 & 0.8237 & 0.4140 \\
$\mathrm{H}(\mathrm{O} 7)$ & 1.3750 & 0.5464 & 0.3059 & $\mathrm{H} 201$ & 1.0680 & 0.8466 & 0.1688 \\
$\mathrm{H} 2$ & 1.1165 & 0.4265 & 0.1751 & $\mathrm{H} 202$ & 1.2090 & 0.8704 & 0.2348 \\
$\mathrm{H} 7$ & 1.4202 & 0.7000 & 0.3221 & $\mathrm{H} 203$ & 1.1558 & 0.8281 & 0.1129 \\
$\mathrm{H} 8$ & 1.3682 & 0.7972 & 0.3144 & $\mathrm{H} 211$ & 0.8787 & 0.2372 & 0.0575 \\
$\mathrm{H} 11$ & 0.9605 & 0.6602 & 0.1161 & $\mathrm{H} 212$ & 0.9479 & 0.2938 & 0.0311 \\
$\mathrm{H} 14$ & 0.9789 & 0.3809 & 0.1124 & $\mathrm{H} 213$ & 0.9413 & 0.2926 & 0.1563 \\
$\mathrm{H} 18$ & 0.7890 & 0.5366 & 0.0194 & $\mathrm{H} 221$ & 0.4877 & 0.5526 & -0.1320 \\
$\mathrm{H} 191$ & 1.0907 & 0.8047 & 0.3351 & $\mathrm{H} 222$ & 0.6136 & 0.5574 & -0.0027 \\
$\mathrm{H} 192$ & 1.1933 & 0.7517 & 0.4065 & $\mathrm{H} 223$ & 0.6180 & 0.5599 & -0.1290 \\
\hline
\end{tabular}




\section{TABELA 6}

PARÂMETROS DE VIBRAÇÃO ANISOTRÓPICA PARA O 5,4'-DIHIDROXI-3',5'DIMETOXI-6,7-(2"',2"'-DIMETILPIRANO) FLAVONA.

\begin{tabular}{|c|c|c|c|c|c|c|}
\hline Átomo & $\mathrm{U}(1,1)$ & $\mathrm{U}(2,2)$ & $\mathrm{U}(3,3)$ & $\mathrm{U}(1,2)$ & $\mathrm{U}(1,3)$ & $U(2,3)$ \\
\hline O1 & $0.021(1)$ & $0.036(1)$ & $0.059(2)$ & $-0.005(1)$ & $0.010(1)$ & $-0.004(1)$ \\
\hline $\mathrm{O} 2$ & $0.038(1)$ & $0.035(2)$ & $0.152(3)$ & $-0.004(1)$ & $0.030(1)$ & $-0.010(2)$ \\
\hline $\mathrm{O} 3$ & $0.022(1)$ & $0.038(1)$ & $0.094(2)$ & $0.001(1)$ & $0.009(1)$ & $0.001(2)$ \\
\hline O4 & $0.026(1)$ & $0.035(1)$ & $0.106(2)$ & $-0.000(1)$ & $0.007(1)$ & $0.007(2)$ \\
\hline O5 & $0.023(1)$ & $0.039(1)$ & $0.103(2)$ & $-0.003(1)$ & $0.008(1)$ & $0.007(2)$ \\
\hline O6 & $0.023(1)$ & $0.043(2)$ & $0.092(2)$ & $0.004(1)$ & $0.010(1)$ & $0.007(2)$ \\
\hline $\mathrm{O} 7$ & $0.022(1)$ & $0.044(2)$ & $0.103(2)$ & $-0.003(1)$ & $0.013(1)$ & $-0.003(2)$ \\
\hline $\mathrm{Cl}$ & $0.029(1)$ & $0.034(2)$ & $0.043(2)$ & $-0.003(1)$ & $0.014(1)$ & $0.002(2)$ \\
\hline $\mathrm{C} 2$ & $0.027(1)$ & $0.034(2)$ & $0.060(2)$ & $-0.001(1)$ & $0.014(1)$ & $0.002(2)$ \\
\hline $\mathrm{C} 3$ & $0.028(1)$ & $0.040(2)$ & $0.048(2)$ & $0.000(2)$ & $0.011(1)$ & $0.007(2)$ \\
\hline $\mathrm{C} 4$ & $0.025(1)$ & $0.039(2)$ & $0.044(2)$ & $-0.003(1)$ & $0.012(1)$ & $-0.001(2)$ \\
\hline $\mathrm{C} 5$ & $0.025(1)$ & $0.040(2)$ & $0.075(3)$ & $-0.003(2)$ & $0.018(1)$ & $-0.002(2)$ \\
\hline $\mathrm{C} 6$ & $0.029(1)$ & $0.040(2)$ & $0.102(3)$ & $-0.006(2)$ & $0.025(2)$ & $-0.008(2)$ \\
\hline $\mathrm{C} 7$ & $0.033(2)$ & $0.042(3)$ & $0.221(6)$ & $-0.007(2)$ & $0.040(2)$ & $-0.018(4)$ \\
\hline $\mathrm{C} 8$ & $0.043(2)$ & $0.051(3)$ & $0.246(6)$ & $-0.020(2)$ & $0.053(3)$ & $-0.034(4)$ \\
\hline C9 & $0.044(2)$ & $0.039(2)$ & $0.116(3)$ & $-0.007(2)$ & $0.031(2)$ & $-0.016(3)$ \\
\hline $\mathrm{C} 10$ & $0.035(2)$ & $0.032(2)$ & $0.093(3)$ & $-0.004(2)$ & $0.026(2)$ & $-0.004(2)$ \\
\hline $\mathrm{C} 11$ & $0.027(1)$ & $0.042(2)$ & $0.077(3)$ & $-0.002(2)$ & $0.021(1)$ & $-0.003(2)$ \\
\hline $\mathrm{C} 12$ & $0.025(1)$ & $0.035(2)$ & $0.055(2)$ & $-0.006(1)$ & $0.015(1)$ & $-0.003(2)$ \\
\hline $\mathrm{C} 13$ & $0.026(1)$ & $0.036(2)$ & 0.042 & $-0.004(1)$ & $0.011(1)$ & $0.000(2)$ \\
\hline $\mathrm{C} 14$ & $0.024(1)$ & $0.040(2)$ & $0.057(2)$ & $-0.002(1)$ & $0.012(1)$ & $0.004(2)$ \\
\hline $\mathrm{C} 15$ & $0.030(1)$ & $0.032(2)$ & $0.065(2)$ & $-0.001(2)$ & $0.013(1)$ & $0.007(2)$ \\
\hline $\mathrm{C} 16$ & $0.022(1)$ & $0.037(2)$ & $0.066(3)$ & $-0.006(2)$ & $0.008(1)$ & $0.003(2)$ \\
\hline $\mathrm{C} 17$ & $0.028(1)$ & $0.036(2)$ & $0.062(2)$ & $0.002(2)$ & $0.014(1)$ & $0.005(2)$ \\
\hline $\mathrm{C} 18$ & $0.029(1)$ & $0.035(2)$ & $0.055(2)$ & $-0.002(2)$ & $0.015(1)$ & $0.000(2)$ \\
\hline $\mathrm{C} 19$ & $0.072(3)$ & $0.150(6)$ & $0.120(5)$ & $-0.018(4)$ & $0.023(3)$ & $-0.014(5)$ \\
\hline $\mathrm{C} 20$ & $0.083(3)$ & $0.056(3)$ & $0.154(5)$ & $-0.019(2)$ & $0.055(3)$ & $-0.000(3)$ \\
\hline $\mathrm{C} 21$ & $0.031(2)$ & $0.037(2)$ & $0.089(3)$ & $0.003(2)$ & $0.009(2)$ & $0.008(2)$ \\
\hline $\mathrm{C} 22$ & $0.034(2)$ & $0.044(2)$ & $0.098(3)$ & $0.006(2)$ & $0.014(2)$ & $-0.003(3)$ \\
\hline
\end{tabular}

A expressão para o parâmetro de vibração anisotrópica é:

$\exp \left[-2 \pi \mathrm{i} 2\left\{\mathrm{~h}^{2} \mathrm{a}^{* 2} \mathrm{U}(1,1)+\mathrm{k}^{2} \mathrm{~b}^{2} \mathrm{U}(2,2)+l^{2} \mathrm{c}^{* 2} \mathrm{U}(3,3)+2 \mathrm{hka} \mathrm{b}^{*} \mathrm{U}(1,2)+2 \mathrm{~h} / \mathrm{a}^{*} \mathrm{c}^{*} \mathrm{U}(1,3)+2 \mathrm{k} / \mathrm{b}^{*} \mathrm{c}^{*} \mathrm{U}(2,3)\right\}\right]$ 
TABELA 7

DISTÂNCIAS INTRAMOLECULARES (Å) COM OS RESPECTIVOS DESVIOS PADRÃO ENTRE PARÊNTESES PARA O 5,4'-DIHIDROXI-3',5'-DIMETOXI-6,7(2",2"'-DIMETILPIRANO) FLAVONA.

\begin{tabular}{|llllll|}
\hline $\mathrm{O} 1$ & $\mathrm{C} 1$ & $1.359(4)$ & $\mathrm{C} 4$ & $\mathrm{C} 12$ & $1.397(4)$ \\
$\mathrm{O} 1$ & $\mathrm{C} 12$ & $1.377(4)$ & $\mathrm{C} 5$ & $\mathrm{C} 6$ & $1.374(6)$ \\
$\mathrm{O} 2$ & $\mathrm{C} 9$ & $1.461(5)$ & $\mathrm{C} 6$ & $\mathrm{C} 7$ & $1.449(6)$ \\
$\mathrm{O} 2$ & $\mathrm{C} 10$ & $1.353(5)$ & $\mathrm{C} 6$ & $\mathrm{C} 10$ & $1.402(5)$ \\
$\mathrm{O} 3$ & $\mathrm{C} 17$ & $1.371(4)$ & $\mathrm{C} 7$ & $\mathrm{C} 8$ & $1.317(7)$ \\
$\mathrm{O} 3$ & $\mathrm{C} 22$ & $1.416(5)$ & $\mathrm{C} 8$ & $\mathrm{C} 9$ & $1.469(7)$ \\
$\mathrm{O} 4$ & $\mathrm{C} 15$ & $1.356(4)$ & $\mathrm{C} 9$ & $\mathrm{C} 19$ & $1.50(1)$ \\
$\mathrm{O} 4$ & $\mathrm{C} 21$ & $1.425(4)$ & $\mathrm{C} 9$ & $\mathrm{C} 20$ & $1.492(7)$ \\
$\mathrm{O} 5$ & $\mathrm{C} 16$ & $1.363(4)$ & $\mathrm{C} 10$ & $\mathrm{C} 11$ & $1.378(5)$ \\
$\mathrm{O} 6$ & $\mathrm{C} 3$ & $1.255(4)$ & $\mathrm{C} 11$ & $\mathrm{C} 12$ & $1.371(5)$ \\
$\mathrm{O} 7$ & $\mathrm{C} 5$ & $1.357(4)$ & $\mathrm{C} 13$ & $\mathrm{C} 14$ & $1.385(5)$ \\
$\mathrm{C} 1$ & $\mathrm{C} 2$ & $1.345(5)$ & $\mathrm{C} 13$ & $\mathrm{C} 18$ & $1.395(5)$ \\
$\mathrm{C} 1$ & $\mathrm{C} 13$ & $1.476(5)$ & $\mathrm{C} 14$ & $\mathrm{C} 15$ & $1.382(5)$ \\
$\mathrm{C} 2$ & $\mathrm{C} 3$ & $1.437(5)$ & $\mathrm{C} 15$ & $\mathrm{C} 16$ & $1.399(5)$ \\
$\mathrm{C} 3$ & $\mathrm{C} 4$ & $1.429(5)$ & $\mathrm{C} 16$ & $\mathrm{C} 17$ & $1.377(5)$ \\
$\mathrm{C} 4$ & $\mathrm{C} 5$ & $1.409(5)$ & $\mathrm{C} 17$ & $\mathrm{C} 18$ & $1.388(5)$ \\
\hline
\end{tabular}

TABELA 8

ÂNGULOS INTRAMOLECULARES (') COM OS DESVIOS PADRÃO ENTRE PARÊNTESES PARA PARA O 5,4'-DIHIDROXI-3',5'-DIMETOXI-6,7-(2",2"'DIMETILPIRANO) FLAVONA.

\begin{tabular}{|llllllll|}
\hline $\mathrm{C} 1$ & $\mathrm{O} 1$ & $\mathrm{C} 12$ & $119.9(2)$ & $\mathrm{O} 2$ & $\mathrm{C} 9$ & $\mathrm{C} 8$ & $111.3(4)$ \\
$\mathrm{C} 9$ & $\mathrm{O} 2$ & $\mathrm{C} 10$ & $120.0(3)$ & $\mathrm{O} 2$ & $\mathrm{C} 9$ & $\mathrm{C} 19$ & $106.4(5)$ \\
$\mathrm{C} 17$ & $\mathrm{O} 3$ & $\mathrm{C} 22$ & $118.0(3)$ & $\mathrm{O} 2$ & $\mathrm{C} 9$ & $\mathrm{C} 20$ & $104.5(3)$ \\
$\mathrm{C} 15$ & $\mathrm{O} 4$ & $\mathrm{C} 21$ & $117.6(3)$ & $\mathrm{C} 8$ & $\mathrm{C} 9$ & $\mathrm{C} 19$ & $111.0(5)$ \\
$\mathrm{O} 1$ & $\mathrm{C} 1$ & $\mathrm{C} 2$ & $122.0(3)$ & $\mathrm{C} 8$ & $\mathrm{C} 9$ & $\mathrm{C} 20$ & $113.3(5)$ \\
$\mathrm{O} 1$ & $\mathrm{C} 1$ & $\mathrm{C} 13$ & $111.8(3)$ & $\mathrm{C} 19$ & $\mathrm{C} 9$ & $\mathrm{C} 20$ & $109.9(5)$ \\
$\mathrm{C} 2$ & $\mathrm{C} 1$ & $\mathrm{C} 13$ & $126.2(3)$ & $\mathrm{O} 2$ & $\mathrm{C} 10$ & $\mathrm{C} 6$ & $121.2(3)$ \\
$\mathrm{C} 1$ & $\mathrm{C} 2$ & $\mathrm{C} 3$ & $121.3(3)$ & $\mathrm{O} 2$ & $\mathrm{C} 10$ & $\mathrm{C} 11$ & $116.0(3)$ \\
$\mathrm{O} 6$ & $\mathrm{C} 3$ & $\mathrm{C} 2$ & $121.8(3)$ & $\mathrm{C} 6$ & $\mathrm{C} 10$ & $\mathrm{C} 11$ & $122.6(4)$ \\
$\mathrm{O} 6$ & $\mathrm{C} 3$ & $\mathrm{C} 4$ & $122.0(3)$ & $\mathrm{C} 10$ & $\mathrm{C} 11$ & $\mathrm{C} 12$ & $117.8(3)$ \\
$\mathrm{C} 2$ & $\mathrm{C} 3$ & $\mathrm{C} 4$ & $116.2(3)$ & $\mathrm{O} 1$ & $\mathrm{C} 12$ & $\mathrm{C} 4$ & $120.8(3)$ \\
$\mathrm{C} 3$ & $\mathrm{C} 4$ & $\mathrm{C} 5$ & $122.9(3)$ & $\mathrm{O} 1$ & $\mathrm{C} 12$ & $\mathrm{C} 11$ & $116.5(3)$ \\
$\mathrm{C} 3$ & $\mathrm{C} 4$ & $\mathrm{C} 12$ & $119.8(3)$ & $\mathrm{C} 4$ & $\mathrm{C} 12$ & $\mathrm{C} 11$ & $122.7(3)$ \\
$\mathrm{C} 5$ & $\mathrm{C} 4$ & $\mathrm{C} 12$ & $117.2(3)$ & $\mathrm{C} 1$ & $\mathrm{C} 13$ & $\mathrm{C} 14$ & $119.3(3)$ \\
$\mathrm{O} 7$ & $\mathrm{C} 5$ & $\mathrm{C} 4$ & $118.0(3)$ & $\mathrm{C} 1$ & $\mathrm{C} 13$ & $\mathrm{C} 18$ & $120.0(3)$ \\
$\mathrm{O} 7$ & $\mathrm{C} 5$ & $\mathrm{C} 6$ & $120.2(3)$ & $\mathrm{C} 14$ & $\mathrm{C} 13$ & $\mathrm{C} 18$ & $120.7(3)$ \\
$\mathrm{C} 4$ & $\mathrm{C} 5$ & $\mathrm{C} 6$ & $121.8(3)$ & $\mathrm{C} 13$ & $\mathrm{C} 14$ & $\mathrm{C} 15$ & $120.2(3)$ \\
$\mathrm{C} 5$ & $\mathrm{C} 6$ & $\mathrm{C} 7$ & $124.5(4)$ & $\mathrm{O} 4$ & $\mathrm{C} 15$ & $\mathrm{C} 14$ & $125.2(3)$ \\
$\mathrm{C} 5$ & $\mathrm{C} 6$ & $\mathrm{C} 10$ & $117.7(3)$ & $\mathrm{O} 4$ & $\mathrm{C} 15$ & $\mathrm{C} 16$ & $115.2(3)$ \\
$\mathrm{C} 7$ & $\mathrm{C} 6$ & $\mathrm{C} 10$ & $117.6(4)$ & $\mathrm{C} 14$ & $\mathrm{C} 15$ & $\mathrm{C} 16$ & $119.5(3)$ \\
$\mathrm{C} 6$ & $\mathrm{C} 7$ & $\mathrm{C} 8$ & $119.7(4)$ & $\mathrm{O} 5$ & $\mathrm{C} 16$ & $\mathrm{C} 15$ & $117.5(3)$ \\
$\mathrm{C} 15$ & $\mathrm{C} 16$ & $\mathrm{C} 17$ & $119.8(3)$ & $\mathrm{C} 16$ & $\mathrm{C} 17$ & $\mathrm{C} 18$ & $121.3(3)$ \\
$\mathrm{O} 3$ & $\mathrm{C} 17$ & $\mathrm{C} 16$ & $114.1(3)$ & $\mathrm{C} 13$ & $\mathrm{C} 18$ & $\mathrm{C} 17$ & $118.5(3)$ \\
$\mathrm{O} 3$ & $\mathrm{C} 17$ & $\mathrm{C} 18$ & $124.6(3)$ & & & & \\
\hline
\end{tabular}


Os planos de mínimos quadrados calculados para a estrutura da molécula 5,4'dihidroxi-3',5'-dimetoxi-6,7(2",2"-dimetilpirano)flavona (tabela 9) mostram que o anel $\gamma$ pirona $(\mathrm{C} 1 / \mathrm{C} 2 / \mathrm{C} 3 / \mathrm{C} 4 / \mathrm{C} 12 / 01)$ e o anel benzênico $\mathrm{A}(\mathrm{C} 4 / \mathrm{C} 5 / \mathrm{C} 6 / \mathrm{C} 10 / \mathrm{C} 11 / \mathrm{C} 12)$ são planares.

O anel benzenico B é totalmente planar com, fazendo um ângulo de $3(1)^{\circ}$, com o plano do anel $\gamma$-pirona.

\section{TABELA 9 \\ EQUAÇÕES DOS PLANOS DE MÍNIMOS QUADRADOS E ÂNGULOS DIEDROS PARA O 5,4'-DIHIDROXI-3',5'-DIMETOXI-6,7-(2', 2''-DIMETILPIRANO) FLAVONA.}

Equação Normalizada do Plano 1: $0.481(1) X+0.049(2) Y-0.8756(7) Z-5.41(3)=0$

Equação Cristalográfica do Plano 1: 6.55(2) X+1.15(4) Y - 13.7(2) Z - 5.41(3) $=0$

Coordenadas Ortogonalizadas dos Átomos no Plano 1

\begin{tabular}{lccrr} 
átomo & $\mathrm{X}$ & $\mathrm{Y}$ & $\mathrm{Z}$ & \multicolumn{1}{c}{ Distância } \\
C1 & 12.8017 & 11.5779 & 1.5006 & $-0.003(4)$ \\
C2 & 13.9027 & 11.0613 & 2.0742 & $-0.001(4)$ \\
C3 & 14.9042 & 11.9072 & 2.6636 & $0.005(4)$ \\
C4 & 14.6500 & 13.3130 & 2.6149 & $-0.006(4)$ \\
C12 & 13.4910 & 13.7884 & 1.9972 & $0.002(4)$ \\
O1 & 12.5781 & 12.9175 & 1.4466 & $0.003(3)$
\end{tabular}

--- outros átomos -----

Chi Squared $=5.4$

$\begin{array}{lllll}\text { O6 } & 15.9384 & 11.4356 & 3.1964 & 0.013(3)\end{array}$

Equação Normalizada do Plano 2: 0.483(2) X+0.044(2) $Y-0.8747(9) Z-5.37(4)=0$

Equação Cristalográfica do Plano 2: 6.58(2) X+1.03(4) $Y-13.7(3) Z-5.37(4)=0$

Coordenadas Ortogonalizadas dos Átomos no Plano 2

$\begin{array}{lcccc}\text { átomo } & \mathrm{X} & \mathrm{Y} & \mathrm{Z} & \text { Distância } \\ \mathrm{C} 4 & 14.6500 & 13.3130 & 2.6149 & -0.004(4) \\ \mathrm{C} 5 & 15.5376 & 14.2700 & 3.1442 & 0.003(4) \\ \text { C6 } & 15.2798 & 15.6177 & 3.0692 & 0.004(5) \\ \text { C10 } & 14.0883 & 16.0200 & 2.4488 & -0.011(5) \\ \text { C11 } & 13.2018 & 15.1248 & 1.8914 & 0.010(4) \\ \text { C12 } & 13.4910 & 13.7884 & 1.9972 & -0.002(4)\end{array}$

---- outros átomos -----

$\begin{array}{lllll}07 & 16.6812 & 13.8256 & 3.7235 & 0.029(2)\end{array}$

Chi Squared $=12.7$ 


\section{TABELA 9 (continuação)}

Equação Normalizada do Plano 3: 0.450(2) X + 0.118(2) Y - 0.885(I) Z - 6.09(5) =0 Equação Cristalográfica do Plano : 6.13(3) X+2.77(5) Y - 13.6(3) Z - 6.09(5) $=0$

Coordenadas Ortogonalizadas dos Átomos no Plano 3

\begin{tabular}{|c|c|c|c|c|}
\hline átomo & $\mathrm{X}$ & $\mathrm{Y}$ & $Z$ & Distância \\
\hline C6 & 15.2798 & I5 6177 & 3.0692 & $-0.085(5)$ \\
\hline $\mathrm{C} 7$ & 16.1931 & 16.6545 & 3.5062 & $0.061(7)$ \\
\hline $\mathrm{C} 8$ & 15.8096 & 17.9142 & 3.4816 & $0.060(7)$ \\
\hline $\mathrm{C} 9$ & 14.4560 & 18.3406 & 3.1008 & $-0.161(5)$ \\
\hline $\mathrm{O} 2$ & 13.7931 & 17.3298 & 2.2798 & $0.148(4)$ \\
\hline $\mathrm{C} 10$ & 14.0883 & 16.0200 & 2.4488 & $-0.024(5)$ \\
\hline \multicolumn{5}{|c|}{ itros átomos -...- } \\
\hline $\mathrm{C} 19$ & 13.5923 & 18.5357 & 4.3120 & $-1.599(7)$ \\
\hline $\mathrm{C} 20$ & 14.4390 & 19.5736 & 2.2611 & $0.721(6)$ \\
\hline
\end{tabular}

Chi Squared $=2939.2$

Equação Normalizada do Plano 4: 0.519(2) X+0.022(2) Y $-0.8544(9) Z-5.58(2)=0$ Equação Cristalográfica do Plano 4: 7.08(2) X+0.51(4) Y $-13.7(2) Z-5.58(2)=0$

Coordenadas Ortogonalizadas dos Átomos no Plano 4

$\begin{array}{ccccc}\text { atomo } & \mathrm{X} & \mathrm{Y} & \mathrm{Z} & \text { Distància } \\ \mathrm{C} 13 & 11.7144 & 10.8153 & 0.8558 & 0.003940 \\ \mathrm{C} 14 & 11.7647 & 9.4317 & 0.8572 & -0.002(4) \\ \text { C15 } & 10.7711 & 8.6993 & 0.2365 & -0.004(4) \\ \text { C16 } & 9.7191 & 9.3659 & -0.4004 & 0.009(4) \\ \text { C17 } & 9.6667 & 10.7415 & -0.3768 & -0.008(4) \\ \text { C18 } & 10.6621 & 11.4898 & 0.2353 & 0.002(4) \\ \text { outros átomos }--. . & & & \\ \text { C1 } & 12.8017 & 11.5779 & 1.5006 & 0.034(4) \\ \text { O5 } & 8.7681 & 8.6042 & -1.0105 & 0.020(3) \\ \text { O4 } & 10.7180 & 7.3460 & 0.1773 & -0.011(3) \\ \text { C21 } & 11.7616 & 6.6154 & 0.8149 & -0.030(4) \\ \text { O3 } & 8.5754 & 11.2709 & -1.0158 & -0.017(3) \\ \text { C22 } & 8.4404 & 12.6801 & -1.0440 & -0.032(5)\end{array}$

Chi Squared $=10.0$

Equação Normalizada do Plano 5:-0.787(5) X - 0.354(7) Y $-0.504(4) Z+19.45(8)=0$ Equação Cristalográfica do Plano5: -10.73(6) X - 8.3(2) Y $-0.7(5) Z+19.45(8)=0$

Coordenadas Ortogonalizadas dos Átomos no Plano 5

$\begin{array}{lccc}\text { átomo } & \mathrm{X} & \mathrm{Y} & \mathrm{Z} \\ \text { C19 } & 13.5923 & 18.5357 & 4.3120 \\ \text { C20 } & 14.4390 & 19.5736 & 2.2611 \\ \text { C9 } & 14.4560 & 18.3406 & 3.1008\end{array}$

\section{Ângulos Diedros Entre Os Planos}

$\begin{array}{crc}\text { Plano No. } & \text { Plano No. } & \text { Ângulos Diedros } \\ 1 & 2 & 0 .(6) \\ 1 & 3 & 4 .(1) \\ 1 & 4 & 3 .(1) \\ 1 & 5 & 87.4(3) \\ 2 & 3 & 5 .(1) \\ 2 & 4 & 3 .(2) \\ 2 & 5 & 87.4(3) \\ 3 & 4 & 7.0(8) \\ 3 & 5 & 87.1(3) \\ 4 & 5 & 89.2(3)\end{array}$


O plano de mínimos quadrados calculado para o anel formado pelos átomos C6/C7/C8/C9/O2/C10, mostrou a quase planaridade deste. As distâncias máximas ao plano ocorrem para $C(9)(-0,161(5) \AA ̊)$ e $O(2)(0,148(4) \AA)$. Este plano faz um ângulo diedro de $4(1)^{0}$ com o anel $\gamma$-pirona.

O plano cálculado para os 2 grupos metil e carbono do anel a que se ligam (C9/C19/C20) faz um ângulo de $87,4(3)^{0}$ com o anel $\gamma$-pirona.

O comprimento da ligação médio calculado para os anéis benzênico A e B são respectivamente de 1,387 e $1,388 \AA$.

Estes valores são próximos aos valores encontrados em anéis aromáticos de flavonas, como por exemplo no composto 5-Hidroxi-6,7,8,3',4',5'-hexametoxi flavona [Rajan e outros, 1987], onde verifica-se o comprimento médio nas ligações de C-C nos anéis benzênicos A e B de 1,39 (1) e 1,40(1) $\AA$ respectivamente. Os valores encontrados são próximos aos valores médios para anéis aromáticos que é de 1,395(3)§̊. [Kennard e outros 1972].

De acordo com a tabela 7 as ligações $C(1)-O(1)$ e $C(12)-O(1)$ são respectivamente de $1,359(4)$ e $1,377(4) \AA$, valores próximos aos valores encontrados para a estrutura de flavonóides descritos por Rossi e outros [1980].

Analisando os comprimentos das ligações $\mathrm{C}(4)-\mathrm{C}(3)$ e $\mathrm{C}(3)-\mathrm{C}(2)$ encontra-se valores de $1,429(5)$ e $1,437(4) \AA$, valores coerentes com as distâncias C-C em uma situação

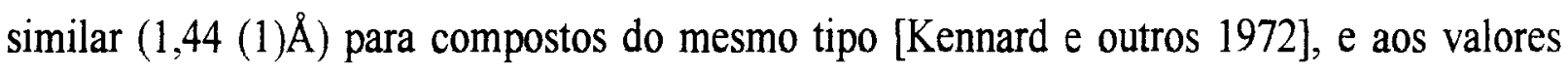
encontrados em outras flavonas [Srinivasan e outros 1986]. Assim por exemplo o comprimento da ligação $C(10)-C(4)$ e $C(4)-C(3)(1,454(4)$ e $1,425(4) \AA)$ no composto 7 hidroxi-2',3',4'-trimetoxi flavona [Molins 1992].correspondem aos valores encontrados neste trabalho. 
Para a ligação C(5)-O(7) a distância de 1,357(4) $\AA$ (tabela 7) é um comprimento de ligação normal para um ligação C (aromático) - O (simples), situação similar à encontrada para uma distância de 1,364(5) $\AA$, observada no 4-bromo-5-hidroxi-flavona [Hayashi, e outros, 1974].

Os átomos de $\mathrm{C}(1)$-C(13) ligam os dois anéis, $\gamma$-pirona e o anel benzênico $\mathrm{B}$, com uma distância de $1,476(5) \AA$. Este valor de distância pode ser comparado com os

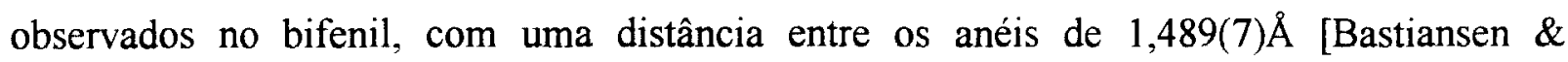

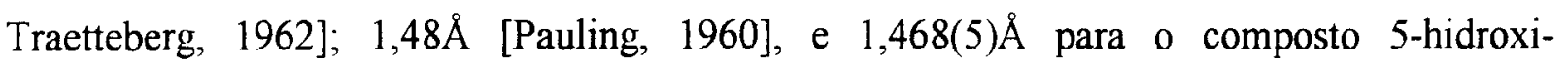
$6,7,8,3^{\prime}, 4^{\prime}, 5^{\prime}$ - hexametoxiflavona [Vijayalakshmi e outros, 1987].

A distância da ligação $\mathrm{C}=\mathrm{O}$ (Carbonila) entre os átomos $\mathrm{C}(3)-\mathrm{O}(6)$ é de $1,255(4) \AA$ (tabela 7) que pode ser comparada ao valor de $1,254 \AA$ encontrado no 4'-bromo-5hidroxiflavona [Hayashi e outros 1974].

Quanto às distâncias de não ligação observa-se que existe uma ligação de hidrogênio entre 0 grupo hidroxila $(\mathrm{O} 7-\mathrm{H})$ ligado ao $\mathrm{C}(5)$ e o oxigênio (O6) ligado ao $\mathrm{C}(3)$. As distâncias de $1,616(3) \AA$ entre o $\mathrm{H}(\mathrm{O} 7)$ e $\mathrm{O}(6)$, e $2,558(3) \AA$ entre $0 \mathrm{O}(7)$ e $\mathrm{O}(6)$ caracterizam que o hidrogênio $\mathrm{H}(\mathrm{O} 7)$, está intramolecularmente ligado ao oxigênio $\mathrm{O}(6)$, estabelecendo assim uma ligação de hidrogênio, com um ângulo $\mathrm{O}(6)-\mathrm{H}(\mathrm{O})-\mathrm{O}(7)$ de $149,5(2)^{\circ}$. Esta ligação de hidrogênio é considerada de força média.

Outra ligação intramolecular, embora mais fraca, pode ser observada entre $\mathrm{O}(5)-\mathrm{H}(05)-\mathrm{O}(3)$, com distância $\mathrm{O}-\mathrm{O}$ de 2,674(4) $\AA$, distância $\mathrm{H}(05)-\mathrm{O}(3)$ de 2,102(3) $\AA$, e ângulo de $113,8(2)^{0}$. Estas ligações intramoleculares podem ser vistas na figura 31 . 


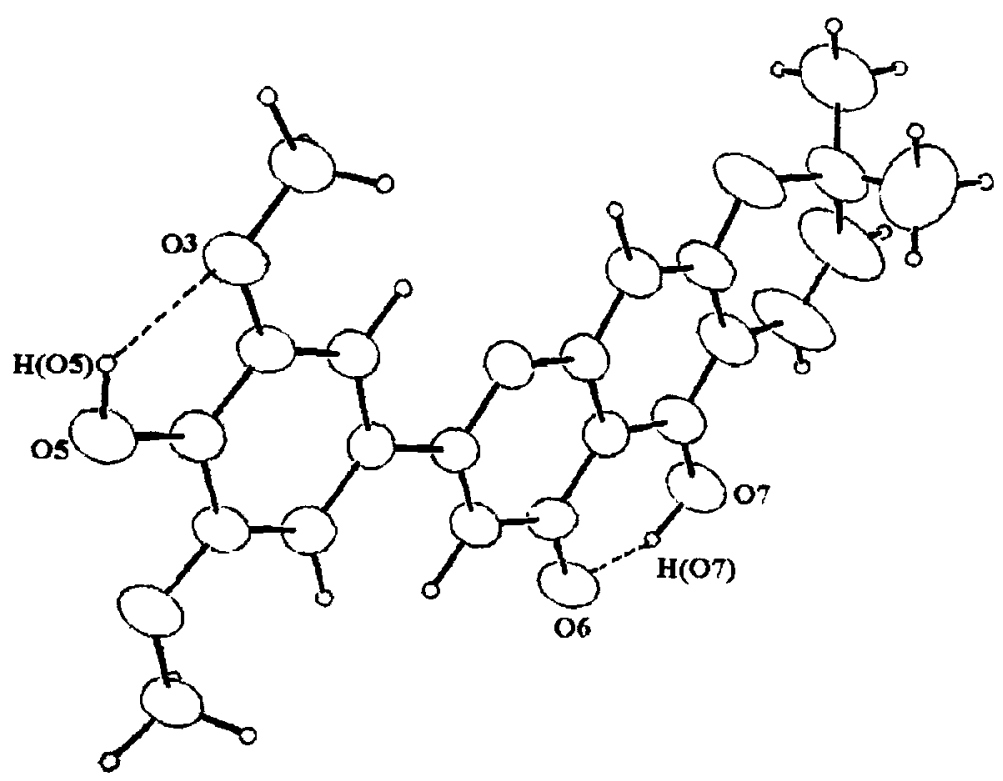

Figura 31 - Representação Ortep da molécula de 5,4'-dihidroxi-3',5'-dimetoxi-6,7(2",2"'-dimetilpirano) flavona. As linhas tracejadas indicam as ligações de hidrogênio intramoleculares.

Além destas ligações intramoleculares o empacotamento cristalino mostra a existência de duas outras ligações, mais fracas, intermoleculares.

Uma é feita compartilhando $\mathrm{H}(05)$, entre $\mathrm{O}(5) \mathrm{e} O(7)$ com operação de simetria $x-1,1-y, z-0,5\left(d_{0-0}=2,830(3) \AA\right)$; a outra é a interação entre $\mathrm{O} 3$ eO7, pela mesma operação de simetria $\left(d_{0-0}=2,992(3) \AA\right)$ compartilhando $\mathrm{H}(\mathrm{O} 7)$.

As distâncias e ângulos envolvidos nas ligações de hidrogênio estão mostradas na tabela 10 e 11 respectivamente.

Tabela 10

DISTÂNCIAS DE LIGAÇÃO DE HIDROGÊNIO (Å) INTRA E INTERMOLECULARES, COM OS RESPECTIVOS DESVIOS PADRÃO ENTRE PARÊNTESES PARA O 5,4'-DIHIDROXI-3',5'-DIMETOXI-6,7-(2",2"'DIMETILPIRANO) FLAVONA.

\begin{tabular}{|ccc|ccc|ccc|}
\hline O6 & O7 & $2,558(4)$ & $\mathrm{O} 6$ & $\mathrm{H}(\mathrm{O} 7)$ & $1,616(3)$ & $\mathrm{O} 7$ & $\mathrm{H}(07)$ & $1,030(2)$ \\
$\mathrm{O} 3$ & $\mathrm{O} 5$ & $2,674(4)$ & $\mathrm{O} 3$ & $\mathrm{H}(05)$ & $2,212(3)$ & $\mathrm{O} 5$ & $\mathrm{H}(05)$ & $1,001(3)$ \\
O5 & $07 *$ & $2,830(3)$ & $\mathrm{O} *$ & $\mathrm{H}(05)$ & $1,924(2)$ & & & \\
O3 & $07 \star$ & $2,992(3)$ & $\mathrm{O} 3$ & $\mathrm{H}(\mathrm{O} 7) \star$ & $2,599(2)$ & & & \\
\hline
\end{tabular}

* operação de simetria: x-1,1-y,z-0,5 
Tabela 11

ÂNGULOS DE LIGAÇ̃̃O DE HIDROGÊNIO ( $\left.{ }^{\circ}\right)$ INTRA E INTERMOLECULARES,

COM OS RESPECTIVOS DESVIOS PADRÃO ENTRE PARÊNTESES PARA O

5,4'-DIHIDROXI-3',5'-DIMETOXI-6,7-(2',2',-DIMETILPIRANO) FLAVONA.

\begin{tabular}{|cccc|clll|}
\hline O6 & $\mathrm{H}(\mathrm{O} 7)$ & $\mathrm{O} 7$ & $149,5(1)$ & $\mathrm{O} 3$ & $\mathrm{H}(\mathrm{O} 5)$ & $\mathrm{O} 5$ & $113,8(2)$ \\
O5 & $\mathrm{H}(\mathrm{O} 5)$ & $\mathrm{O} *$ & $147,9(2)$ & O3 & $\mathrm{HO}(7) \star$ & $\mathrm{O}$ * & $102,2(1)$ \\
\hline
\end{tabular}

* operação de simetria: $\mathrm{x}-1,1-\mathrm{y}, \mathrm{z}-0,5$

As ligações intermoleculares produzem o efeito de uma cadeia infinita de moléculas colocadas ao longo da direção [101] e geradas pelo espelho c, como mostrado na figura 32 .

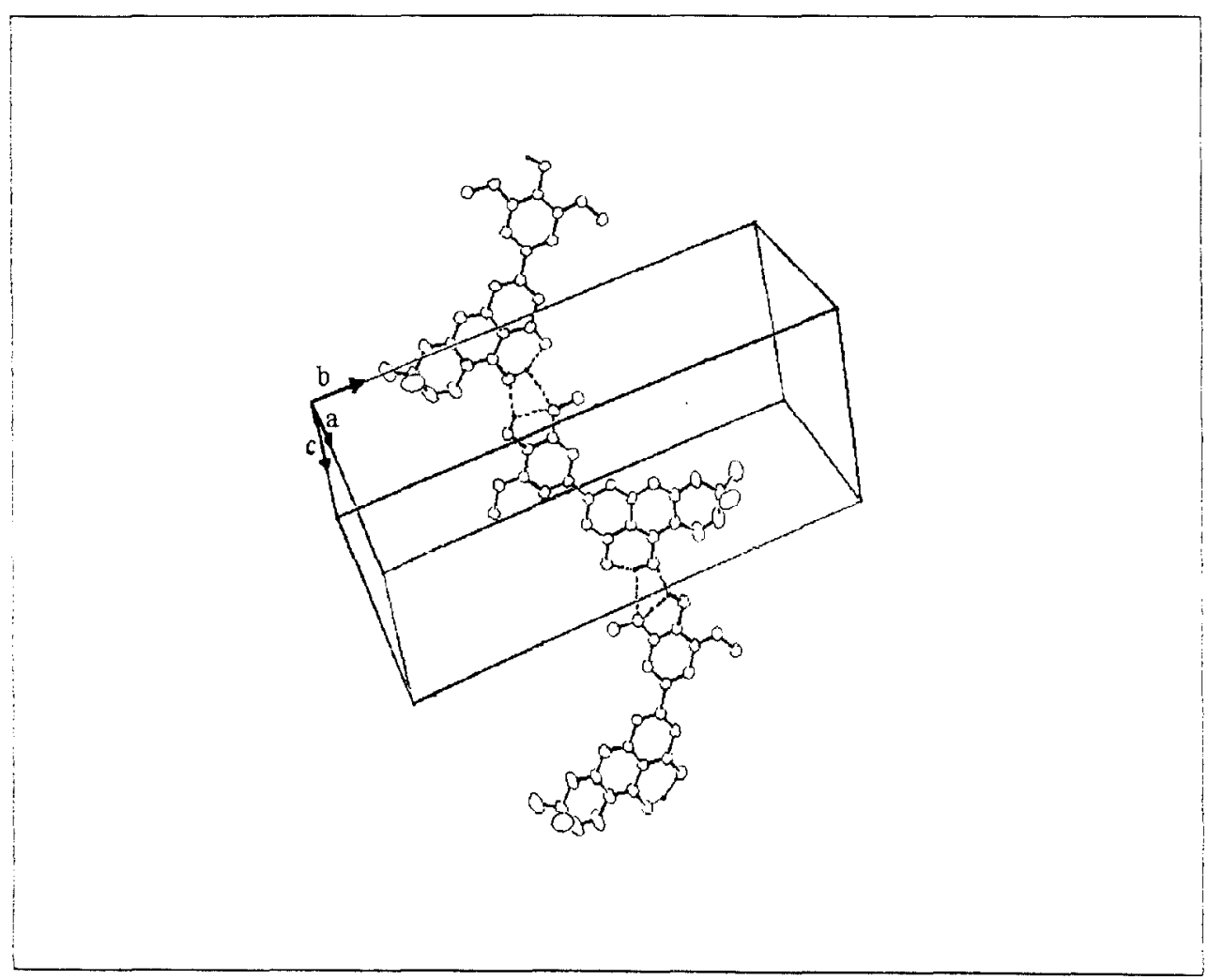

Figura 32 - Representação ORTEP das moléculas com orientação relativa à cela unitária, formando cadeia a longo da direção [101]. As ligações de hidrogênio intra e intermoleculares estão representadas por linhas tracejadas. 


\section{REFERÊNCIAS BIBLIOGRÁFICAS}

Arruda, A. C., Souza, J.P.I. de, Souza, M.B.C. \& Arruda, M.S.P.; $16^{\mathrm{a}}$ Reunião Anual da Sociedade Brasileira de Química. Caxambú, MG, maio 1993.

Azároff, L.V.; Elements of X-ray crystallography. (1968), New York, Mac Graw-Hill Book Company.

Bastiansen, O. \& Traetteberg, M; Tetrahedron, (1962), 17, 147.

Borges, S.B.; Elementos de Cristalografia. (1980), Lisboa, Fundação Calouste Gulbernian.

Bunn, C. W., Cristais se Papel na Natureza e na Ciência. (1972), São Paulo, Editora da Universidade de São Paulo

Cochran, W: Acta Cryst. (1955), 8, 473.

Cochran, W. \& Woolfson, M.M.; Acta Cryst. (1955), 8 ,1.

Cromer,D.T. \& Waber, J.T.;.International Tables For X-Ray Cryatallography. (1974) IV, London, The Kynoch Press.

Cullity, B.D.; Elements of x-ray diffraction In Detection of x-ray. (1967), New York, Addisson-Wesley.

Enraf -Nonius; Molen Crystal Structures Analysis. (1990), Vol.I, Holland.

Geismann,T.A. \& Hinreiner,E.; Bot.Rev. (1952),18, 77.

Germain, G., Main, P. \& Woofson, M.M.; Acta Cryst. (1970), B26, 274.

Germain, G. \& Woofson, M.M.; Acta Cryst. (1968), B24, 91.

Gooddwin, T.W.; Chemistry and Biochemistry of Plants Pigments. (1965), London, Academic Press.

Harker, D. \& Kasper.; Acta Cryst. (1948), 1, 70.

Hauptmann H. \& Karle, J.; Monograph 3 (1953), Pittsburgh, Polycrystal Book Service.

Hayashi, T., Kaway, S., Ohno, T., Iitaka,Y \& Akimoto, T.; Chem. Pharm. Bull (1974)22, 1219.

Hull, S.E. \& Irwin, M. J.; Acta Cryst. (1978), A34 , 863

Hukins, W.L.D.; X-ray Diffraction by Disordered and Ordered Systems. (1981), New York, Pergamon Press.

Ikan, R.; Natural Products, A Laboratory Guide. (1991), New York, Academic Press, Inc..

Johnson, C.K.;. ORTEP: A FORTRAN Thermal- ellipsoid Plot Program for Crystal Structure Illustration. (1965), Oak Ridge National Laboratory, Tennessee, USA. 
Karle, J.; Escola Latino Americana de Cristalografia: Métodos Diretos (Notas de Aula), (1976), Cap. II, São Carlos, SP, Brasil.

Karle, J \& Hauptman, H.; Acta Cryst. (1950), 3, 181.

Karle, J \& Hauptman, H.; Acta Cryst. (1956), 9, 635.

Karle, J . \& Karle I.L.; Acta Cryst. (1964), 17, 835.

Karle, J . \& Karle I.L.; Acta Cryst. (1966), 21, 849.

Kennard, D.J., Watson, F.H., Allen,N.W., Isaacs, W.D., Motherwell, S., Patterson, R.C. \& Town, W.G.; Molecular Structures and Dimensions. (1972), A1, Utrech: Oosthoek.

Killean, R.C.G. \& Lawrence, J.L.; Acta Cryst. (1969), B25, 1750.

Main. P.; Escola Latino Americana de Cristalografia. Métodos Diretos (Notas de Aula). (1976) Cap. 6, São Carlos, SP, Brasil.

Morris, S.J. \& Thomson, R.H.; J. Insect Physiol. (1963),9, 391.

Pauling, L.; The Nature of The Chemical Bond. (1960), Cornell Univ. Press.

Rossi, M., Cantrell, J.S., Farber, A. J.,Dyott, T.,Carrel, H.L. Glusker, J.P.; Cancer Res. (1980), 40, 2774.

Sands, D.E.; Introduction to Crystallography. (1993), New York, Dover Publications, Inc..

Santos, L. da S., Silva, E.O. da \& Lobato, C.M. da L.; XXXV Congresso Brasileiro de Química. Salvador, Ba, setembro (1995)

Sayre, D.; Acta Cryst. (1952), 5, 60.

Schenk, H.; Direct Methods of Solving Crystal Structures. (1991), New York, Plenum Press Stewart, R.F., Davidson, E.R. \& Simpson W.T.; J.Chem. Phys.: (1965), 42, 3175.

Stout, G.H. \& Jensen, L.H.; X-ray Structure Determination: A Practical Guide. (1989), New York, John Wiley \& Sons, Inc.

Vainshtein, B.K.; Modern Crystallography Symmetry of Crystals, Methods of Structural Crystallography. (1981), New York, Springer-Verlag Berlin Heidlberg New York. Vijayalakshma, J., Rajan, S.S. \& Srinivasan, R.; Acta Cryst. (1987), C43, 1998.

Willis, B.T.M. and Pryor, A.W.; Thermal Vibrations in Crvstallography. (1975), Cambridge, Cambridge University Press.

Woolfson, M.M.; Escola Latino Americana de Cristalografia: Métodos Diretos №tas de Aula), (1976), Cap. XV, São Carlos, SP, Brasil. 


\title{
APENDICE 1
}

\author{
TABELA A1
}

FATORES DE ESTRUTURA OBSERVADOS E CALCULADOS PARA O 5,4'-DIHIDROXI-3',5'-DIMETOXI- 6,7 -(2', 2'"-DIMETILPIRANO)FLAVONA 
Reflexões observadas $I \geq 3 \sigma(I)$

Values of $10 *$ Fobs and $10 *$ Fcalc

Page

\begin{tabular}{|c|c|c|c|c|c|c|c|c|c|}
\hline $\mathrm{H}$ & $\mathrm{L}$ & Fobs & Ecalc & $\operatorname{Sig} F$ & $\mathrm{H}$ & K & Fobs & Fcalc & SigF \\
\hline - & - & ---- & ---- & ---- & - & - & ---- & ----- & ---- \\
\hline 8 & $0-16$ & 326 & 328 & 9 & 10 & $18-12$ & 200 & 177 & 11 \\
\hline 7 & $1-16$ & 135 & 102 & 15 & 9 & $19-12$ & 124 & 93 & 14 \\
\hline 7 & $11-15$ & 115 & 102 & 17 & 5 & $1-11$ & 208 & 269 & 8 \\
\hline 11 & $7-14$ & 113 & 105 & 15 & 7 & $1-11$ & 209 & 226 & 8 \\
\hline 6 & $12-14$ & 122 & 106 & 17 & 9 & $1-11$ & 180 & 142 & 9 \\
\hline 5 & $1-13$ & 161 & 118 & 13 & 15 & $1-11$ & 152 & 156 & 13 \\
\hline 6 & $2-13$ & 242 & 244 & 10 & 4 & $2-11$ & 210 & 216 & 8 \\
\hline 3 & $3-13$ & 101 & 60 & 15 & 8 & $2-11$ & 135 & 123 & 10 \\
\hline 11 & $3-13$ & 116 & 117 & 15 & 10 & $2-11$ & 163 & 170 & 12 \\
\hline 2 & $4-13$ & 110 & 89 & 15 & 1 & $3-11$ & 198 & 149 & 9 \\
\hline 12 & $6-13$ & 137 & 134 & 13 & 5 & $3-11$ & 215 & 189 & 8 \\
\hline 11 & $7-13$ & 124 & 124 & 14 & 7 & $3-11$ & 222 & 216 & 8 \\
\hline 7 & $11-13$ & 192 & 182 & 11 & 11 & $3-11$ & 193 & 183 & 1: \\
\hline 2 & $16-13$ & 158 & 148 & 14 & 15 & $3-11$ & 324 & 312 & 9 \\
\hline 3 & $17-13$ & 204 & 196 & 12 & 10 & $4-11$ & 172 & 149 & 11 \\
\hline 4 & $0-12$ & 111 & 90 & 14 & 14 & $4-11$ & 168 & 156 & 12 \\
\hline 6 & $0-12$ & 1002 & 1027 & 14 & 1 & $5-11$ & 245 & 303 & 9 \\
\hline 16 & $0-12$ & 258 & 275 & 10 & 13 & $5-11$ & 132 & 112 & 12 \\
\hline 1 & $1-12$ & 121 & 74 & 14 & 15 & $5-11$ & 188 & 192 & 11 \\
\hline 5 & $1-12$ & 228 & 204 & 9 & 14 & $6-11$ & 168 & 167 & 12 \\
\hline 7 & $1-12$ & 332 & 331 & 7 & 1 & $7-11$ & 188 & 170 & 11 \\
\hline 11 & $1-12$ & 120 & 91 & 16 & 9 & $7-11$ & 116 & 115 & 15 \\
\hline 6 & $2-12$ & 458 & 508 & 7 & 2 & $8-11$ & 155 & 138 & 13 \\
\hline 16 & $2-12$ & 212 & 212 & 11 & 6 & $8-11$ & 165 & 175 & 12 \\
\hline 5 & $3-12$ & 209 & 227 & 10 & 5 & $9-11$ & 125 & 135 & 15 \\
\hline 15 & $3-12$ & 131 & 132 & 15 & 6 & $10-11$ & 193 & 187 & 11 \\
\hline 6 & $4-12$ & 152 & 105 & 12 & 5 & $11-11$ & 286 & 277 & 9 \\
\hline 16 & $4-12$ & 161 & 155 & 14 & 12 & $12-11$ & 143 & 135 & 14 \\
\hline 1 & $5-12$ & 150 & 174 & 12 & 5 & $13-11$ & 190 & 162 & 12 \\
\hline 15 & $5-12$ & 253 & 243 & 11 & 11 & $15-11$ & 162 & 147 & 13 \\
\hline 2 & $6-12$ & 193 & 176 & 12 & 10 & $16-11$ & 172 & 159 & 12 \\
\hline 1 & $7-12$ & 139 & 109 & 12 & 12 & $16-11$ & 162 & 150 & 13 \\
\hline 11 & $7-12$ & 144 & 161 & 13 & 3 & $17-11$ & 118 & 111 & 15 \\
\hline 10 & $8-12$ & 103 & 105 & 16 & 9 & $17-11$ & 124 & 108 & 14 \\
\hline 11 & $13-12$ & 188 & 195 & 11 & 2 & $18-11$ & 115 & 150 & 17 \\
\hline 2 & $14-12$ & 111 & 102 & 17 & 10 & $18-11$ & 299 & 294 & 9 \\
\hline 12 & $14-12$ & 189 & 172 & 11 & 12 & $18-11$ & 108 & 80 & 16 \\
\hline 1 & $15-12$ & 311 & 285 & 9 & 9 & $19-11$ & 195 & 188 & 11 \\
\hline 11 & $15-12$ & 264 & 258 & 9 & 2 & $20-11$ & 117 & 113 & 18 \\
\hline 2 & $16-12$ & 201 & 212 & 12 & 8 & $20-11$ & 181 & 189 & 12 \\
\hline 12 & $16-12$ & 147 & 148 & 15 & 9 & $21-11$ & 159 & 141 & 12 \\
\hline 1 & $17-12$ & 221 & 218 & 11 & 4 & $0-10$ & 151 & 136 & 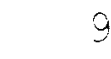 \\
\hline 11 & $17-12$ & 125 & 133 & 15 & 6 & $0-10$ & 227 & 196 & 7 \\
\hline 2 & $18-12$ & 132 & 118 & 16 & 14 & $0-10$ & 262 & 245 & \\
\hline
\end{tabular}


Reflexões não observadas $I<3 \sigma$ (I)

Values of $10 *$ Fobs and $10 *$ Fcalc

Page 2

\begin{tabular}{|c|c|c|c|c|c|c|c|c|c|}
\hline $\mathrm{H}$ & $\mathrm{L}$ & Fobs & Ecalc & $\operatorname{SigF}$ & $\mathrm{H}$ & I & Fobs & Fcalc & $\operatorname{Sig} F$ \\
\hline- & - & -- & -ーーー- & ---- & - & - & ---- & ----- & ---- \\
\hline 13 & $5-15$ & 0 & 33 & 92 & 12 & $0-14$ & -97 & 4 & 36 \\
\hline 2 & $6-15$ & 59 & 104 & 64 & 14 & $0-14$ & -20 & 16 & 41 \\
\hline 4 & $6-15$ & 53 & 20 & 28 & 1 & $1-14$ & -54 & 56 & 53 \\
\hline 6 & $6-15$ & 52 & 48 & 28 & 3 & $1-14$ & 60 & 4 & 22 \\
\hline 8 & $6-15$ & -98 & 15 & 47 & 5 & $1-14$ & -117 & 83 & 35 \\
\hline 10 & $6-15$ & -104 & 7 & 39 & 7 & $1-14$ & -105 & 28 & 34 \\
\hline 12 & $6-15$ & -112 & 17 & 41 & 9 & $1-14$ & 80 & 22 & 18 \\
\hline 3 & $7-15$ & -64 & 70 & 55 & 11 & $1-14$ & 0 & 47 & 92 \\
\hline 5 & $7-15$ & -63 & 6 & 52 & 13 & $1-14$ & -83 & 43 & 44 \\
\hline 7 & $7-15$ & 32 & 41 & 79 & 15 & $1-14$ & 35 & 4 & 73 \\
\hline 9 & $7-15$ & -62 & 9 & 49 & 2 & $2-14$ & -28 & 8 & 35 \\
\hline 11 & $7-15$ & -111 & 28 & 36 & 4 & $2-14$ & 68 & 36 & 21 \\
\hline 13 & $7-15$ & 49 & 5 & 62 & 6 & $2-14$ & -68 & 6 & 21 \\
\hline 2 & $8-15$ & 70 & 13 & 58 & 8 & $2-14$ & -58 & 11 & 52 \\
\hline 4 & $8-15$ & 0 & 38 & 100 & 10 & $2-14$ & 95 & 39 & 16 \\
\hline 6 & $8-15$ & -58 & 7 & 26 & 12 & $2-14$ & 55 & 61 & 55 \\
\hline 10 & $8-15$ & -49 & 25 & 27 & 14 & $2-14$ & 48 & 35 & 63 \\
\hline 12 & $8-15$ & -60 & 8 & 25 & 1 & $3-14$ & -113 & 29 & 35 \\
\hline 3 & $9-15$ & 49 & 4 & 65 & 3 & $3-14$ & 0 & 37 & 92 \\
\hline 5 & $9-15$ & -78 & 20 & 48 & 5 & $3-14$ & -31 & 47 & 34 \\
\hline 7 & $9-15$ & -78 & 59 & 52 & 7 & $3-14$ & 30 & 53 & 71 \\
\hline 9 & $9-15$ & -71 & 13 & 51 & 9 & $3-14$ & -48 & 10 & 26 \\
\hline 11 & $9-15$ & -73 & 2 & 52 & 11 & $3-14$ & -74 & 46 & 53 \\
\hline 4 & $10-15$ & 49 & 29 & 70 & 13 & $3-14$ & 0 & 1 & 91 \\
\hline 6 & $10-15$ & 49 & 3 & 32 & 15 & $3-14$ & -100 & 35 & 48 \\
\hline 8 & $10-15$ & 87 & 86 & 21 & 2 & $4-14$ & 56 & 28 & 59 \\
\hline 10 & $10-15$ & 50 & 27 & 27 & 4 & $4-14$ & -29 & 27 & 71 \\
\hline 12 & $10-15$ & 61 & 25 & 49 & 6 & $4-14$ & 43 & 48 & 61 \\
\hline 3 & $11-15$ & -134 & 4 & 41 & 8 & $4-14$ & 84 & 9 & 18 \\
\hline 5 & $11-15$ & -73 & 20 & 53 & 10 & $4-14$ & -51 & 19 & 51 \\
\hline 9 & $11-15$ & -113 & 17 & 36 & 12 & $4-14$ & 0 & 61 & 100 \\
\hline 11 & $11-15$ & -66 & 26 & 50 & 14 & $4-14$ & 49 & 12 & 65 \\
\hline 4 & $12-15$ & -66 & 5 & 55 & 1 & $5-14$ & 66 & 22 & 50 \\
\hline 6 & $12-15$ & 55 & 28 & 31 & 3 & $5-14$ & -42 & 1 & 59 \\
\hline 8 & $12-15$ & -80 & 42 & 54 & 5 & $5-14$ & -123 & 6 & 32 \\
\hline 10 & $12-15$ & 59 & 8 & 57 & 7 & $5-14$ & -69 & 27 & 21 \\
\hline 5 & $13-15$ & -82 & 25 & 47 & 9 & $5-14$ & 80 & 10 & 17 \\
\hline 7 & $13-15$ & -66 & 67 & 53 & 11 & $5-14$ & -101 & 29 & 41 \\
\hline 9 & $13-15$ & 95 & 6 & 20 & 13 & $5-14$ & -31 & 61 & 79 \\
\hline 2 & $0-14$ & 32 & 1 & 68 & 2 & $6-14$ & -53 & 27 & 54 \\
\hline 4 & $0-14$ & -59 & 27 & 48 & 4 & $6-14$ & -11 & 26 & 49 \\
\hline 6 & $0-14$ & 73 & 36 & 47 & 6 & $6-14$ & 0 & 18 & 95 \\
\hline 8 & $0-14$ & 52 & 75 & 56 & 8 & $6-14$ & 0 & 4 & 93 \\
\hline 10 & $0-14$ & -41 & 61 & 60 & 10 & $6-14$ & -86 & 8 & 41 \\
\hline
\end{tabular}


Reflexões observadas $I \geq 3 \sigma$ (I)

Values of $10^{*}$ Fobs and $10^{*} \mathrm{FCalC}$

Page 3

\begin{tabular}{|c|c|c|c|c|c|c|c|c|c|c|}
\hline $\mathrm{H}$ & $\mathrm{L}$ & Fobs & Ecalc & $\operatorname{Sig} F$ & $\mathrm{H}$ & K & $\mathrm{L}$ & Fobs & Fcalc & $\operatorname{Sig} F$ \\
\hline - & - & ---- & ---- & ---- & - & - & - & ---- & ---- & ---- \\
\hline 1315 & -9 & 170 & 155 & 12 & 2 & 6 & -8 & 128 & 150 & 9 \\
\hline 1016 & -9 & 219 & 221 & 10 & 4 & 6 & -8 & 246 & 274 & 6 \\
\hline 1216 & -9 & 220 & 217 & 10 & 6 & 6 & -8 & 265 & 260 & 6 \\
\hline $\begin{array}{ll}1 & 1\end{array}$ & -9 & 391 & 385 & 7 & 8 & 6 & -8 & 211 & 202 & 7 \\
\hline $\begin{array}{ll}7 & 17\end{array}$ & -9 & 184 & 164 & 9 & 10 & 6 & -8 & 200 & 214 & 8 \\
\hline 111 & -9 & 177 & 173 & 11 & 3 & 7 & -8 & 235 & 219 & 7 \\
\hline 818 & -9 & 113 & 120 & 14 & 7 & 7 & -8 & 153 & 145 & 9 \\
\hline 1018 & -9 & 337 & 310 & 8 & 9 & 7 & -8 & 306 & 335 & 6 \\
\hline 11 & -9 & 157 & 183 & 12 & 11 & 7 & -8 & 118 & 96 & 14 \\
\hline 71 & -9 & 214 & 199 & 9 & 15 & 7 & -8 & 166 & 153 & 12 \\
\hline 42 & -9 & 97 & 47 & 14 & 4 & 8 & -8 & 281 & 252 & 6 \\
\hline 82 & -9 & 231 & 232 & 9 & 6 & 8 & -8 & 165 & 149 & 8 \\
\hline $\begin{array}{ll}32 \\
\end{array}$ & $3-9$ & 133 & 147 & 14 & 14 & 8 & -8 & 211 & 219 & 10 \\
\hline 2 & -8 & 477 & 446 & 7 & 1 & 9 & -8 & 110 & 105 & 11 \\
\hline 4 & -8 & 3051 & 3165 & 11 & 5 & 9 & -8 & 200 & 192 & 7 \\
\hline 8 & -8 & 258 & 236 & 6 & 7 & 9 & -8 & 109 & 120 & 12 \\
\hline 10 & -8 & 105 & 124 & 12 & 11 & 9 & -8 & 137 & 157 & 14 \\
\hline 14 & -8 & 606 & 618 & 10 & 2 & 10 & -8 & 422 & 432 & 6 \\
\hline 1 & -8 & 136 & 125 & 8 & 4 & 10 & -8 & 246 & 259 & 7 \\
\hline 3 & $L-8$ & 404 & 411 & 6 & 6 & 10 & -8 & 213 & 211 & 7 \\
\hline 5 & -8 & 571 & 567 & 8 & 7 & 11 & -8 & 88 & 90 & 13 \\
\hline 7 & -8 & 293 & 272 & 5 & 2 & 12 & -8 & 130 & 137 & 10 \\
\hline 9 & -8 & 268 & 244 & 6 & 4 & 12 & -8 & 139 & 96 & 10 \\
\hline 13 & $1-8$ & 170 & 170 & 12 & 6 & 12 & -8 & 121 & 96 & 10 \\
\hline 2 & $2-8$ & 312 & 303 & 5 & 8 & 12 & -8 & 189 & 199 & 8 \\
\hline 4 & $2-8$ & 1106 & 1156 & 11 & 1 & 13 & -8 & 94 & 100 & 14 \\
\hline 6 & $2-8$ & 134 & 140 & 8 & 3 & 13 & -8 & 186 & 167 & 8 \\
\hline 8 & $2-8$ & 232 & 216 & 6 & 7 & 13 & -8 & 130 & 129 & 10 \\
\hline 12 & $2-8$ & 148 & 126 & 12 & 9 & 13 & -8 & 308 & 297 & 8 \\
\hline 14 & $2-8$ & 448 & 431 & 7 & 11 & 13 & -8 & 280 & 269 & 8 \\
\hline 1 & $3-8$ & 171 & 162 & 7 & 4 & 14 & -8 & 145 & 132 & 10 \\
\hline 3 & $3-8$ & 315 & 327 & 5 & 6 & 14 & -8 & 138 & 103 & 9 \\
\hline 11 & $3-8$ & 284 & 288 & 7 & 10 & 14 & -8 & 354 & 354 & 8 \\
\hline 13 & $3-8$ & 180 & 192 & 12 & 9 & 15 & -8 & 515 & 505 & 8 \\
\hline 4 & $4-8$ & 269 & 230 & 5 & 6 & 16 & -8 & 140 & 157 & 12 \\
\hline 6 & $4-8$ & 159 & 177 & 7 & 8 & 16 & -8 & 264 & 267 & 9 \\
\hline 8 & $4-8$ & 113 & 93 & 11 & 10 & 16 & -8 & 229 & 254 & 9 \\
\hline 14 & $4-8$ & 329 & 326 & 8 & 14 & 16 & -8 & 125 & 102 & 13 \\
\hline 1 & $5-8$ & 241 & 239 & 6 & 1 & 17 & -8 & 115 & 81 & 15 \\
\hline 3 & $5-8$ & 338 & 318 & 5 & 5 & 17 & -8 & 110 & 122 & 16 \\
\hline 7 & $5-8$ & 148 & 133 & 9 & 7 & 17 & -8 & 113 & 85 & 15 \\
\hline 9 & $5-8$ & 175 & 180 & 9 & 9 & 17 & -8 & 224 & 228 & 9 \\
\hline 13 & $5-8$ & 584 & 571 & 9 & 13 & 17 & -8 & 109 & 85 & 15 \\
\hline 15 & $5-8$ & 137 & 172 & 14 & 6 & 18 & -8 & 136 & 101 & 12 \\
\hline
\end{tabular}


Reflexões observadas $I \geq 3 \sigma$ (I)

Values of $10 *$ Eobs and $10 *$ Ecalc

Page 4

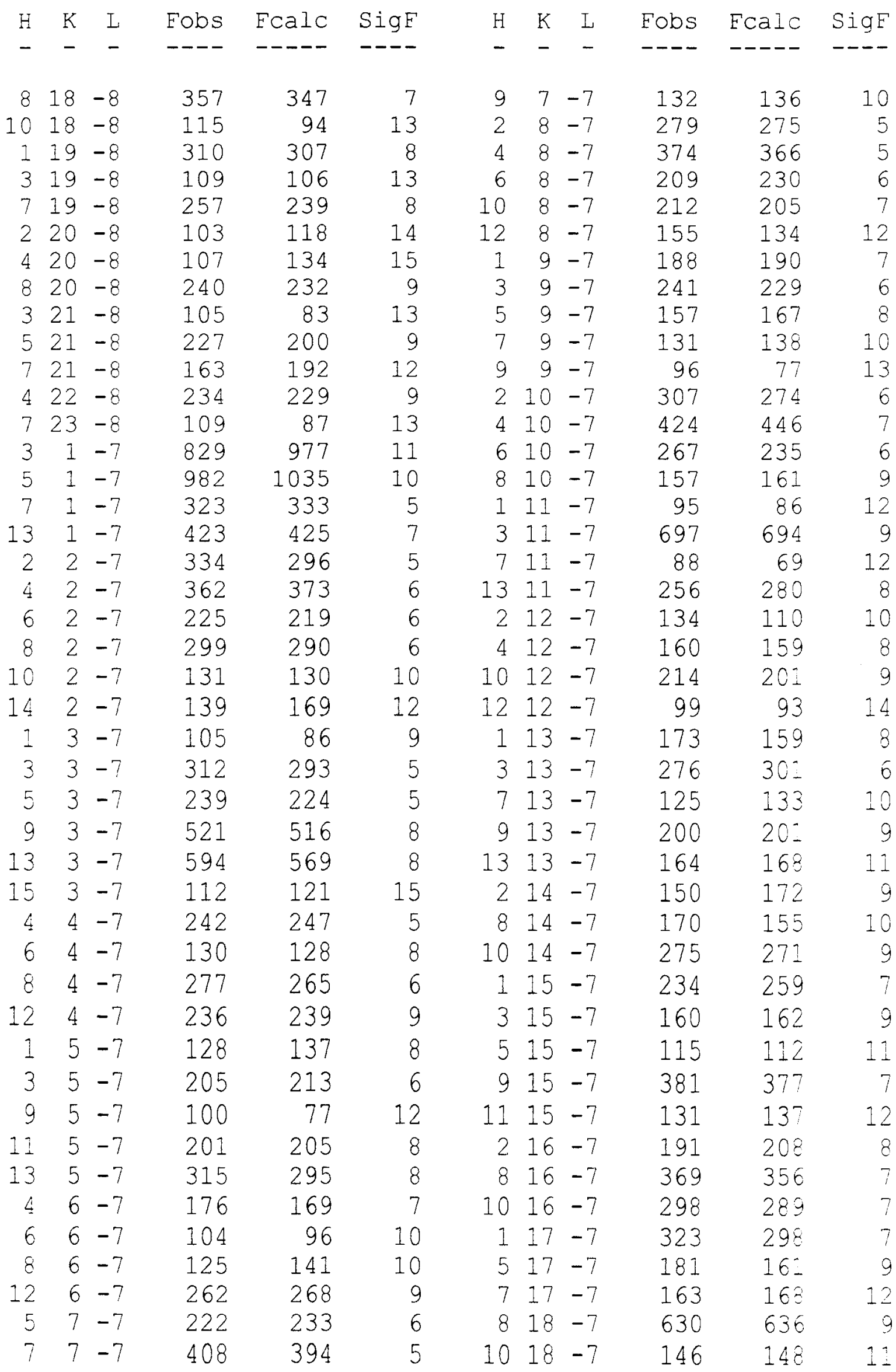


Reflexões observadas $I \geq 3 \sigma$ (I)

Values of $10 *$ Fobs and $10 *$ Fcalc

Page 5

\begin{tabular}{|c|c|c|c|c|c|c|c|c|c|c|c|}
\hline $\mathrm{H}$ & K & $I$ & Fobs & Ecalc & $\operatorname{Sig} F$ & $\mathrm{H}$ & $\mathrm{K}$ & L & Fobs & Fcalc & $\operatorname{Sig} F$ \\
\hline- & - & - & --- & ---- & ---- & - & - & - & ---- & -- & ---- \\
\hline 1 & 19 & -7 & 124 & 122 & 14 & 10 & 6 & -6 & 163 & 156 & 8 \\
\hline 7 & 19 & -7 & 342 & 335 & 7 & 12 & 6 & -6 & 253 & 258 & 9 \\
\hline 6 & 20 & -7 & 381 & 385 & 7 & 1 & 7 & -6 & 240 & 251 & 5 \\
\hline 3 & 21 & -7 & 102 & 99 & 14 & 5 & 7 & -6 & 327 & 322 & 5 \\
\hline 7 & 21 & -7 & 227 & 215 & 8 & 7 & 7 & -6 & 562 & 578 & 9 \\
\hline 8 & 22 & -7 & 120 & 106 & 12 & 9 & 7 & -6 & 429 & 424 & 6 \\
\hline 3 & 23 & -7 & 132 & 112 & 12 & 13 & 7 & -6 & 248 & 239 & 8 \\
\hline 5 & 23 & -7 & 177 & 165 & 10 & 15 & 7 & -6 & 110 & 115 & 14 \\
\hline 2 & 0 & -6 & 735 & 830 & 10 & 4 & 8 & -6 & 150 & 140 & 7 \\
\hline 6 & 0 & -6 & 288 & 249 & 5 & 6 & 8 & -6 & 161 & 202 & 7 \\
\hline 8 & 0 & -6 & 231 & 231 & 6 & 8 & 8 & -6 & 379 & 367 & 5 \\
\hline 10 & 0 & -6 & 92 & 109 & 12 & 10 & 8 & -6 & 227 & 226 & 7 \\
\hline 12 & 0 & -6 & 423 & 425 & 7 & 14 & 8 & -6 & 165 & 159 & 11 \\
\hline 14 & 0 & -6 & 386 & 381 & 7 & 3 & 9 & -6 & 377 & 330 & 5 \\
\hline 1 & 1 & -6 & 364 & 360 & 6 & 5 & 9 & -6 & 329 & 291 & 5 \\
\hline 3 & 1 & -6 & 144 & 197 & 7 & 7 & 9 & -6 & 408 & 420 & 6 \\
\hline 7 & 1 & -6 & 307 & 319 & 5 & 4 & 10 & -6 & 230 & 250 & 6 \\
\hline 11 & 1 & -6 & 128 & 145 & 11 & 6 & 10 & -6 & 164 & 167 & 7 \\
\hline 13 & 1 & -6 & 307 & 304 & 9 & 12 & 10 & -6 & 147 & 115 & 12 \\
\hline 2 & 2 & -6 & 297 & 306 & 5 & 1 & 11 & -6 & 295 & 294 & 5 \\
\hline 4 & 2 & -6 & 302 & 315 & 5 & 3 & 11 & -6 & 103 & 91 & 10 \\
\hline 6 & 2 & -6 & 422 & 453 & 7 & 5 & 11 & -6 & 168 & 168 & 7 \\
\hline 8 & 2 & -6 & 308 & 306 & 5 & 7 & 11 & -6 & 210 & 199 & 6 \\
\hline 16 & 2 & -6 & 175 & 170 & 12 & 11 & 11 & -6 & 260 & 253 & 9 \\
\hline 1 & 3 & -6 & 385 & 382 & 5 & 2 & 12 & -6 & 672 & 665 & 9 \\
\hline 3 & 3 & -6 & 229 & 187 & 5 & 4 & 12 & -6 & 669 & 665 & 9 \\
\hline 5 & 3 & -6 & 277 & 292 & 5 & 6 & 12 & -6 & 304 & 294 & 5 \\
\hline 7 & 3 & -6 & 312 & 309 & 5 & 8 & 12 & -6 & 157 & 164 & 9 \\
\hline 11 & 3 & -6 & 193 & 205 & 8 & 12 & 12 & -6 & 178 & 162 & 10 \\
\hline 13 & 3 & -6 & 325 & 333 & 8 & 14 & 12 & -6 & 205 & 190 & 10 \\
\hline 2 & 4 & -6 & 164 & 134 & 6 & 9 & 13 & -6 & 328 & 320 & 7 \\
\hline 4 & 4 & -6 & 227 & 224 & 5 & 2 & 14 & -6 & 296 & 290 & 6 \\
\hline 6 & 4 & -6 & 116 & 132 & 9 & 4 & 14 & -6 & 194 & 186 & 7 \\
\hline 8 & 4 & -6 & 470 & 487 & 8 & 10 & 14 & -6 & 508 & 497 & 8 \\
\hline 10 & 4 & -6 & 142 & 152 & 10 & 12 & 14 & -6 & 245 & 253 & 9 \\
\hline 12 & 4 & -6 & 347 & 350 & 7 & 7 & 15 & -6 & 162 & 163 & 8 \\
\hline 14 & 4 & -6 & 293 & 286 & 8 & 9 & 15 & -6 & 392 & 368 & 7 \\
\hline 1 & 5 & -6 & 94 & 106 & 10 & 13 & 15 & -6 & 106 & 100 & 14 \\
\hline 7 & 5 & -6 & 598 & 601 & 9 & 10 & 16 & -6 & 143 & 135 & 11 \\
\hline 9 & 5 & -6 & 478 & 500 & 7 & 12 & 16 & -6 & 164 & 181 & 12 \\
\hline 11 & 5 & -6 & 264 & 258 & 7 & 9 & 17 & -6 & 342 & 349 & 8 \\
\hline 13 & 5 & -6 & 372 & 383 & 8 & 4 & 18 & -6 & 199 & 156 & 9 \\
\hline 2 & 6 & -6 & 157 & 123 & 7 & 6 & 18 & -6 & 335 & 328 & 8 \\
\hline 6 & 6 & -6 & 338 & 336 & 5 & 8 & 18 & -6 & 159 & 125 & 11 \\
\hline
\end{tabular}


Reflexões não observadas $I<3 \sigma$ (I)

Values of $10 *$ Fobs and $10 *$ Ecalc

Page 6

\begin{tabular}{|c|c|c|c|c|c|c|c|c|c|}
\hline $\mathrm{H}$ & K I & Fobs & Ecalc & $\operatorname{SigF}$ & $\mathrm{H}$ & $I$ & Fobs & Fcalc & SigF \\
\hline - & - & ---- & $-\cdots$ & --- & - & - & ---- & ---- & ---- \\
\hline 4 & $16-12$ & 59 & 83 & 26 & 7 & $5-11$ & 67 & 12 & 17 \\
\hline 6 & $16-12$ & 27 & 3 & 38 & 9 & $5-11$ & 49 & 3 & 27 \\
\hline 8 & $16-12$ & -82 & 59 & 48 & 11 & $5-11$ & -27 & 64 & 71 \\
\hline 10 & $16-12$ & 73 & 101 & 51 & 2 & $6-11$ & 27 & 44 & 75 \\
\hline 3 & $17-12$ & -44 & 1 & 28 & 4 & $6-11$ & 70 & 113 & 47 \\
\hline 5 & $17-12$ & 81 & 1 & 48 & 6 & $6-11$ & 54 & 27 & 21 \\
\hline 7 & $17-12$ & -59 & 15 & 24 & 8 & $6-11$ & -50 & 7 & 25 \\
\hline 9 & $17-12$ & -36 & 15 & 32 & 10 & $6-11$ & -102 & 36 & 40 \\
\hline 4 & $18-12$ & 13 & 3 & 50 & 12 & $6-11$ & -112 & 10 & 35 \\
\hline 6 & $18-12$ & 77 & 19 & 19 & 16 & $6-11$ & -57 & 7 & 60 \\
\hline 8 & $18-12$ & -78 & 51 & 42 & 3 & $7-11$ & -32 & 39 & 35 \\
\hline 5 & $19-12$ & -32 & 51 & 68 & 5 & $7-11$ & 90 & 43 & 15 \\
\hline 7 & $19-12$ & -108 & 20 & 38 & 7 & $7-11$ & 78 & 58 & 18 \\
\hline 4 & $20-12$ & -12 & 59 & 49 & 11 & $7-11$ & 84 & 23 & 49 \\
\hline 6 & $20-12$ & 89 & 75 & 20 & 13 & $7-11$ & 76 & 37 & 20 \\
\hline 8 & $20-12$ & -105 & 49 & 44 & 15 & $7-11$ & -129 & 24 & 34 \\
\hline 2 & $0-11$ & -81 & 0 & 41 & 4 & $8-11$ & 77 & 108 & 54 \\
\hline 4 & $0-11$ & -50 & 0 & 53 & 8 & $8-11$ & 0 & 72 & 92 \\
\hline 6 & $0-11$ & -109 & 0 & 32 & 10 & $8-11$ & -55 & 24 & 28 \\
\hline 8 & $0-11$ & 55 & 0 & 21 & 12 & $8-11$ & 69 & 36 & 43 \\
\hline 10 & $0-11$ & -110 & 0 & 36 & 14 & $8-11$ & 90 & 55 & 17 \\
\hline 12 & $0-11$ & 51 & 0 & 56 & 1 & $9-11$ & 58 & 33 & 57 \\
\hline 14 & $0-11$ & -121 & 0 & 30 & 3 & $9-11$ & -104 & 18 & 36 \\
\hline 16 & $0-11$ & 49 & 0 & 57 & 7 & $9-11$ & 47 & 38 & 62 \\
\hline 1 & $1-11$ & 66 & 12 & 48 & 9 & $9-11$ & -99 & 5 & 39 \\
\hline 3 & $1-11$ & -62 & 5 & 43 & 11 & $9-11$ & 12 & 46 & 48 \\
\hline 11 & $1-11$ & -82 & 36 & 43 & 13 & $9-11$ & 37 & 3 & 32 \\
\hline 13 & $1-11$ & -65 & 5 & 50 & 2 & $10-11$ & 89 & 79 & 20 \\
\hline 2 & $2-11$ & -50 & 28 & 22 & 4 & $10-11$ & 69 & 35 & 52 \\
\hline 6 & $2-11$ & 87 & 68 & 15 & 8 & $10-11$ & 100 & 105 & 18 \\
\hline 12 & $2-11$ & -98 & 29 & 40 & 10 & $10-11$ & -80 & 68 & 46 \\
\hline 14 & $2-11$ & 62 & 33 & 24 & 12 & $10-11$ & 66 & 57 & 22 \\
\hline 16 & $2-11$ & 92 & 103 & 20 & 14 & $10-11$ & -55 & 24 & 51 \\
\hline 3 & $3-11$ & -57 & 30 & 50 & 1 & $11-11$ & 86 & 5 & 15 \\
\hline 9 & $3-11$ & 0 & 2 & 90 & 3 & $11-11$ & 59 & 69 & 28 \\
\hline 13 & $3-11$ & -135 & 15 & 29 & 7 & $11-11$ & 70 & 39 & 55 \\
\hline 2 & $4-11$ & -25 & 84 & 69 & 9 & $11-11$ & -94 & 67 & 47 - \\
\hline 4 & $4-11$ & 52 & 15 & 52 & 11 & $11-11$ & 0 & 11 & 95 \\
\hline 6 & $4-11$ & -35 & 54 & 59 & 13 & $11-11$ & -76 & 5 & 4 \\
\hline 8 & $4-11$ & 53 & 42 & 49 & 15 & 11-11 & -88 & 121 & 52 \\
\hline 12 & $4-11$ & 30 & 42 & 72 & 2 & $12-12$ & -157 & 17 & 25 \\
\hline 16 & $4-11$ & 60 & 64 & 27 & 4 & $12-11$ & 48 & 49 & 3 \\
\hline 3 & $5-11$ & 13 & 14 & 42 & 6 & $12-11$ & 73 & 95 & 2 \\
\hline 5 & $5-11$ & 52 & 92 & 54 & 8 & $12-11$ & 57 & 19 & 51 \\
\hline
\end{tabular}


Reflexões observadas $I \geq 3 \sigma$ (I)

Values of $10 *$ Fobs and $10 *$ Fcalc

Page 7

\begin{tabular}{|c|c|c|c|c|c|c|c|c|c|c|c|}
\hline $\mathrm{H}$ & K & $\mathrm{L}$ & Fobs & Fcalc & SigF & $\mathrm{H}$ & $\mathrm{K}$ & L & Fobs & Ecalc & SigF \\
\hline - & - & - & ---- & ---- & --- & - & - & - & ---- & ----- & ---- \\
\hline 9 & 17 & -5 & 215 & 217 & 10 & 15 & 3 & -4 & 118 & 99 & 14 \\
\hline 11 & 17 & -5 & 142 & 120 & 10 & 2 & 4 & -4 & 730 & 739 & 9 \\
\hline 4 & 18 & -5 & 171 & 157 & 9 & 4 & 4 & -4 & 181 & 160 & 5 \\
\hline 6 & 18 & -5 & 214 & 212 & 8 & 8 & 4 & -4 & 208 & 189 & 6 \\
\hline 8 & 18 & -5 & 589 & 579 & 9 & 10 & 4 & -4 & 238 & 237 & 7 \\
\hline 5 & 19 & -5 & 409 & 411 & 7 & 12 & 4 & -4 & 638 & 620 & 9 \\
\hline 7 & 19 & -5 & 130 & 130 & 14 & 14 & 4 & -4 & 113 & 107 & 13 \\
\hline 9 & 19 & -5 & 95 & 95 & 14 & 1 & 5 & -4 & 486 & 496 & 7 \\
\hline 2 & 20 & -5 & 132 & 161 & 13 & 3 & 5 & -4 & 458 & 455 & 6 \\
\hline 6 & 20 & -5 & 459 & 445 & 7 & 5 & 5 & -4 & 397 & 411 & 6 \\
\hline 3 & 21 & -5 & 116 & 129 & 14 & 7 & 5 & -4 & 191 & 185 & 6 \\
\hline 2 & 22 & -5 & 154 & 184 & 10 & 11 & 5 & -4 & 893 & 932 & 10 \\
\hline 4 & 22 & -5 & 158 & 150 & 10 & 13 & 5 & -4 & 304 & 323 & 8 \\
\hline 1 & 23 & -5 & 219 & 258 & 9 & 15 & 5 & -4 & 129 & 132 & 13 \\
\hline 2 & 24 & -5 & 104 & 89 & 14 & 2 & 6 & -4 & 506 & 510 & 7 \\
\hline 3 & 25 & -5 & 114 & 106 & 12 & 4 & 6 & -4 & 615 & 620 & 7 \\
\hline 7 & 25 & -5 & 105 & 113 & 15 & 6 & 6 & -4 & 606 & 577 & 7 \\
\hline 2 & 0 & -4 & 8219 & 8199 & 27 & 8 & 6 & -4 & 284 & 288 & 5 \\
\hline 4 & 0 & -4 & 698 & 673 & 9 & 16 & 6 & -4 & 169 & 138 & 12 \\
\hline 6 & 0 & -4 & 621 & 621 & 7 & 1 & 7 & -4 & 521 & 545 & 7 \\
\hline 8 & 0 & -4 & 429 & 414 & 6 & 3 & 7 & -4 & 409 & 414 & 7 \\
\hline 10 & 0 & -4 & 212 & 217 & 7 & 5 & 7 & -4 & 265 & 293 & 5 \\
\hline 12 & 0 & -4 & 1258 & 1245 & 15 & 7 & 7 & -4 & 710 & 696 & 8 \\
\hline 14 & 0 & -4 & 167 & 185 & 10 & 9 & 7 & -4 & 181 & 193 & 7 \\
\hline 16 & 0 & -4 & 203 & 207 & 11 & 13 & 7 & -4 & 232 & 252 & 9 \\
\hline 1 & 1 & -4 & 608 & 614 & 8 & 2 & 8 & -4 & 649 & 692 & 10 \\
\hline 3 & 1 & -4 & 1026 & 1023 & 8 & 4 & 8 & -4 & 171 & 190 & 5 \\
\hline 5 & 1 & -4 & 730 & 710 & 9 & 8 & 8 & -4 & 342 & 350 & 6 \\
\hline 7 & 1 & -4 & 563 & 551 & 8 & 10 & 8 & -4 & 122 & 89 & 10 \\
\hline 9 & 1 & -4 & 175 & 186 & 7 & 12 & 8 & -4 & 443 & 439 & 7 \\
\hline 11 & 1 & -4 & 212 & 231 & 7 & 14 & 8 & -4 & 117 & 124 & 13 \\
\hline 2 & 2 & -4 & 2106 & 2164 & 8 & 1 & 9 & -4 & 104 & 108 & 8 \\
\hline 4 & 2 & -4 & 205 & 242 & 4 & 3 & 9 & -4 & 322 & 330 & 5 \\
\hline 6 & 2 & -4 & 288 & 343 & 4 & 5 & 9 & -4 & 268 & 250 & - \\
\hline 8 & 2 & -4 & 84 & 85 & 11 & 7 & 9 & -4 & 172 & 179 & 6 \\
\hline 10 & 2 & -4 & 179 & 182 & 7 & 9 & 9 & -4 & 168 & 179 & $a$ \\
\hline 12 & 2 & -4 & 604 & 592 & 9 & 2 & 10 & -4 & 420 & 408 & 6 \\
\hline 16 & 2 & -4 & 145 & 132 & 13 & 4 & 10 & -4 & 682 & 632 & 0 \\
\hline 1 & 3 & -4 & 519 & 514 & 6 & 10 & 10 & -4 & 98 & 98 & 12 \\
\hline 3 & 3 & -4 & 488 & 475 & 6 & 12 & 10 & -4 & 221 & 229 & \\
\hline 5 & 3 & -4 & 239 & 254 & 4 & 1 & 11 & -4 & 145 & 144 & 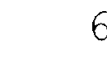 \\
\hline 7 & 3 & -4 & 127 & 147 & 7 & 3 & 11 & -4 & 73 & 67 & 11 \\
\hline 9 & 3 & -4 & 620 & 616 & 9 & 5 & 11 & -4 & 244 & 235 & \\
\hline 11 & 3 & -4 & 299 & 281 & 6 & 9 & 11 & -4 & 109 & 129 & 11 \\
\hline
\end{tabular}


Reflexões observadas $I \geq 3 \sigma$ (I)

Values of $10 *$ Fobs and $10 *$ Fcalc

Page 8

\begin{tabular}{|c|c|c|c|c|c|c|c|c|c|c|c|}
\hline $\mathrm{H}$ & K & $\mathrm{L}$ & Fobs & Ecalc & $\operatorname{Sig} E$ & $\mathrm{H}$ & $\mathrm{K}$ & L & Fobs & Fcalc & SigF \\
\hline- & - & - & ---- & ----- & --- & - & - & - & --- & --ー-- & ---- \\
\hline 15 & 11 & -4 & 249 & 238 & 9 & 6 & 26 & -4 & 173 & 146 & \\
\hline 2 & 12 & -4 & 212 & 198 & 5 & 1 & 27 & -4 & 123 & 122 & \\
\hline 4 & 12 & -4 & 272 & 280 & 5 & 5 & 27 & -4 & 114 & 96 & \\
\hline 6 & 12 & -4 & 203 & 225 & 6 & 1 & 1 & -3 & 2069 & 2099 & \\
\hline 1 & 13 & -4 & 313 & 299 & 5 & 3 & 1 & -3 & 2029 & 1998 & \\
\hline 5 & 13 & -4 & 265 & 253 & 5 & 5 & 1 & -3 & 1033 & 988 & \\
\hline 7 & 13 & -4 & 282 & 294 & 6 & 7 & 1 & -3 & 162 & 156 & \\
\hline 9 & 13 & -4 & 458 & 454 & 6 & 11 & 1 & -3 & 606 & 628 & \\
\hline 2 & 14 & -4 & 265 & 248 & 5 & 13 & 1 & -3 & 228 & 244 & \\
\hline 4 & 14 & -4 & 188 & 180 & 6 & 2 & 2 & -3 & 1418 & 1490 & \\
\hline 8 & 14 & -4 & 504 & 511 & 7 & 4 & 2 & -3 & 430 & 444 & \\
\hline 10 & 14 & -4 & 202 & 211 & 10 & 6 & 2 & -3 & 339 & 306 & \\
\hline 12 & 14 & -4 & 98 & 72 & 14 & 8 & 2 & -3 & 192 & 187 & \\
\hline 5 & 15 & -4 & 123 & 122 & 9 & 12 & 2 & -3 & 193 & 201 & \\
\hline 7 & 15 & -4 & 803 & 825 & 10 & 14 & 2 & -3 & 103 & 110 & \\
\hline 11 & 15 & -4 & 166 & 161 & 10 & 16 & 2 & -3 & 134 & 140 & 1 \\
\hline 4 & 16 & -4 & 243 & 267 & 6 & 1 & 3 & -3 & 388 & 429 & \\
\hline 6 & 16 & -4 & 458 & 483 & 7 & 3 & 3 & -3 & 505 & 542 & \\
\hline 8 & 16 & -4 & 349 & 353 & 7 & 7 & 3 & -3 & 1137 & 1105 & \\
\hline 12 & 16 & -4 & 173 & 162 & 11 & 11 & 3 & -3 & 973 & 972 & \\
\hline 1 & 17 & -4 & 166 & 198 & 8 & 13 & 3 & -3 & 169 & 177 & \\
\hline 3 & 17 & -4 & 258 & 273 & 6 & 2 & 4 & -3 & 141 & 143 & \\
\hline 5 & 17 & -4 & 241 & 245 & 6 & 4 & 4 & -3 & 210 & 223 & \\
\hline 7 & 17 & -4 & 377 & 366 & 6 & 6 & 4 & -3 & 683 & 640 & \\
\hline $1 i$ & 17 & -4 & 146 & 161 & 11 & 8 & 4 & -3 & 242 & 233 & \\
\hline 4 & 18 & -4 & 180 & 195 & 8 & 10 & 4 & -3 & 238 & 267 & \\
\hline 6 & 18 & -4 & 542 & 557 & 9 & 14 & 4 & -3 & 192 & 189 & \\
\hline 8 & 18 & -4 & 275 & 271 & 8 & 1 & 5 & -3 & 253 & 257 & \\
\hline 5 & 19 & -4 & 536 & 531 & 9 & 5 & 5 & -3 & 218 & 270 & \\
\hline 7 & 19 & -4 & 145 & 146 & 12 & 9 & 5 & -3 & 356 & 351 & \\
\hline 9 & 19 & -4 & 175 & 164 & 10 & 11 & 5 & -3 & 207 & 211 & \\
\hline 11 & 19 & -4 & 100 & 94 & 15 & 13 & 5 & -3 & 142 & 127 & \\
\hline 2 & 20 & -4 & 173 & 147 & 9 & 2 & 6 & -3 & 296 & 247 & \\
\hline 4 & 20 & -4 & 176 & 165 & 10 & 4 & 6 & -3 & 405 & 417 & \\
\hline 6 & 20 & -4 & 323 & 327 & 8 & 6 & 6 & -3 & 191 & 187 & \\
\hline 8 & 20 & -4 & 113 & 107 & 12 & 10 & 6 & -3 & 357 & 364 & \\
\hline 1 & 21 & -4 & 210 & 224 & 9 & 1 & 7 & -3 & 158 & 177 & \\
\hline 3 & 21 & -4 & 335 & 353 & 7 & 3 & 7 & -3 & 412 & 413 & \\
\hline 5 & 21 & -4 & 168 & 144 & 10 & 5 & 7 & -3 & 927 & 940 & \\
\hline 2 & 22 & -4 & 384 & 413 & 7 & 7 & 7 & -3 & 391 & 402 & \\
\hline 4 & 22 & -4 & 130 & 167 & 12 & 9 & 7 & -3 & 108 & 118 & \\
\hline 5 & 23 & -4 & 158 & 195 & 11 & 13 & 7 & -3 & 195 & 184 & \\
\hline 9 & 23 & -4 & 113 & 85 & 13 & 15 & 7 & -3 & 146 & 157 & \\
\hline 2 & 26 & -4 & 123 & 129 & 12 & 2 & 8 & -3 & 790 & 830 & \\
\hline
\end{tabular}


Reflexões observadas $I \geq 3 \sigma$ (I)

Values of $10^{*}$ Fobs and $10^{*}$ Fcalc

Page 9

\begin{tabular}{|c|c|c|c|c|c|c|c|c|c|c|c|}
\hline $\mathrm{H}$ & $\mathrm{K}$ & L & Fobs & Fcalc & SigF & $\mathrm{H}$ & K & L & Fobs & Fcalc & $\operatorname{Sig} F$ \\
\hline- & - & - & ---- & ----- & ---- & - & - & - & ---- & ----- & ---- \\
\hline 4 & 8 & -3 & 514 & 510 & 7 & 9 & 17 & -3 & 108 & 116 & 15 \\
\hline 6 & 8 & -3 & 175 & 161 & 6 & 6 & 18 & -3 & 1004 & 1015 & 14 \\
\hline 8 & 8 & -3 & 359 & 329 & 5 & 8 & 18 & -3 & 186 & 161 & 10 \\
\hline 10 & 8 & -3 & 176 & 184 & 7 & 10 & 18 & -3 & 167 & 173 & 10 \\
\hline 14 & 8 & -3 & 144 & 159 & 12 & 12 & 18 & -3 & 146 & 131 & 12 \\
\hline 1 & 9 & -3 & 262 & 205 & 4 & 5 & 19 & -3 & 509 & 512 & 8 \\
\hline 3 & 9 & -3 & 513 & 535 & 7 & 7 & 19 & -3 & 178 & 177 & 10 \\
\hline 5 & 9 & -3 & 314 & 319 & 4 & 4 & 20 & -3 & 543 & 538 & 9 \\
\hline 7 & 9 & -3 & 87 & 77 & 11 & 1 & 21 & -3 & 174 & 168 & 10 \\
\hline 9 & 9 & -3 & 180 & 184 & 7 & 5 & 21 & -3 & 233 & 215 & 8 \\
\hline 2 & 10 & -3 & 779 & 764 & 10 & 7 & 21 & -3 & 124 & 102 & 11 \\
\hline 4 & 10 & -3 & 291 & 288 & 4 & 2 & 22 & -3 & 111 & 135 & 15 \\
\hline 10 & 10 & -3 & 136 & 131 & 10 & 4 & 22 & -3 & 168 & 148 & 11 \\
\hline 1 & $1 I$ & -3 & 1491 & 1544 & 10 & 6 & 22 & -3 & 176 & 194 & 10 \\
\hline 3 & 11 & -3 & 243 & 250 & 5 & 1 & 23 & -3 & 167 & 167 & 9 \\
\hline 5 & 11 & -3 & 217 & 186 & 5 & 3 & 23 & -3 & 305 & 316 & 7 \\
\hline 7 & 11 & -3 & 324 & 334 & 5 & 4 & 24 & -3 & 93 & 96 & 14 \\
\hline 11 & 11 & -3 & 362 & 406 & 8 & 6 & 24 & -3 & 132 & 120 & 11 \\
\hline 13 & 11 & -3 & 126 & 149 & 13 & 7 & 25 & -3 & 106 & 100 & 14 \\
\hline 2 & 12 & -3 & 165 & 124 & 6 & 4 & 26 & -3 & 112 & 100 & 13 \\
\hline 4 & 12 & -3 & 272 & 227 & 5 & 6 & 26 & -3 & 153 & 136 & 12 \\
\hline 6 & 12 & -3 & 273 & 257 & 5 & 3 & 27 & -3 & 97 & 77 & 14 \\
\hline 8 & 12 & -3 & 144 & 151 & 8 & 5 & 27 & -3 & 196 & 151 & 10 \\
\hline 1 & 13 & -3 & 372 & 360 & 6 & 2 & 0 & -2 & 641 & 691 & 6 \\
\hline 3 & 13 & -3 & 120 & 91 & 7 & 4 & 0 & -2 & 460 & 412 & 6 \\
\hline 5 & 13 & -3 & 326 & 334 & 5 & 6 & 0 & -2 & 961 & 929 & 10 \\
\hline 11 & 13 & -3 & 230 & 249 & 8 & 8 & 0 & -2 & 243 & 259 & 5 \\
\hline 4 & 14 & -3 & 108 & 120 & 9 & 10 & 0 & -2 & 687 & 697 & 10 \\
\hline 6 & 14 & -3 & 203 & 191 & 6 & 12 & 0 & -2 & 502 & 502 & - \\
\hline 8 & 14 & -3 & 619 & 625 & 8 & 14 & 0 & -2 & 192 & 183 & 9 \\
\hline 10 & 14 & -3 & 101 & 115 & 15 & 1 & 1 & -2 & 250 & 280 & 3 \\
\hline 1 & 15 & -3 & 327 & 333 & 5 & 3 & 1 & -2 & 316 & 316 & \\
\hline 3 & 15 & -3 & 309 & 284 & 5 & 5 & 1 & -2 & 962 & 937 & 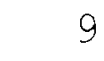 \\
\hline 5 & 15 & -3 & 225 & 229 & 6 & 7 & 1 & -2 & 104 & 103 & \\
\hline 7 & 15 & -3 & 683 & 704 & 10 & 9 & 1 & -2 & 265 & 258 & \\
\hline 9 & 15 & -3 & 345 & 346 & 7 & 11 & 1 & -2 & 597 & 600 & \\
\hline 11 & 15 & -3 & 232 & 231 & 8 & 2 & 2 & -2 & 737 & 767 & \\
\hline 4 & 16 & -3 & 129 & 134 & 8 & 4 & 2 & -2 & 1167 & 1207 & \\
\hline 6 & 16 & -3 & 673 & 673 & 10 & 6 & 2 & -2 & 102 & 57 & \\
\hline 8 & 16 & -3 & 468 & 470 & 6 & 12 & 2 & -2 & 153 & 151 & 1 \\
\hline 1 & 17 & -3 & 248 & 249 & 6 & 14 & 2 & -2 & 258 & 265 & \\
\hline 3 & 17 & -3 & 237 & 240 & 6 & 1 & 3 & -2 & 192 & 230 & \\
\hline 5 & 17 & -3 & 283 & 260 & 6 & 3 & 3 & -2 & 989 & 996 & \\
\hline 7 & 17 & -3 & 185 & 165 & 10 & 5 & 3 & -2 & 420 & 450 & \\
\hline
\end{tabular}


Reflexões não observadas $I<3 \sigma(I)$

Values of 10*Fobs and 10*Fcalc

Page 10

\begin{tabular}{|c|c|c|c|c|c|c|c|c|c|c|}
\hline K & I & Fobs & Ecalc & $\operatorname{Sig} F$ & $\mathrm{H}$ & K & L & Eobs & Ecalc & Sig \\
\hline - & - & ---- & ----- & ---- & - & - & - & ---- & ----- & \\
\hline 622 & -9 & 70 & 65 & 21 & 3 & 11 & -8 & -75 & 28 & \\
\hline 822 & -9 & -121 & 12 & 38 & 5 & 11 & -8 & -44 & 19 & \\
\hline $10 \quad 22$ & -9 & -100 & 32 & 38 & 9 & 11 & -8 & -73 & 11 & \\
\hline 123 & -9 & 60 & 37 & 52 & 11 & 11 & -8 & 39 & 62 & \\
\hline 523 & -9 & 55 & 23 & 23 & 13 & 11 & -8 & -73 & 2 & \\
\hline $\begin{array}{ll}7 & 23\end{array}$ & -9 & -56 & 23 & 50 & 15 & 11 & -8 & -65 & 5 & \\
\hline 923 & -9 & -47 & 10 & 52 & 10 & 12 & -8 & 56 & 18 & \\
\hline 224 & -9 & 94 & 107 & 19 & 12 & 12 & -8 & -71 & 24 & \\
\hline 424 & -9 & -73 & 83 & 51 & 14 & 12 & -8 & -63 & 15 & \\
\hline 624 & -9 & -87 & 35 & 41 & 5 & 13 & -8 & -79 & 45 & \\
\hline 6 & -8 & 0 & 25 & 74 & 13 & 13 & -8 & 0 & 15 & \\
\hline 12 & -8 & -37 & 67 & 61 & 15 & 13 & -8 & 48 & 7 & \\
\hline 16 & -8 & 84 & 93 & 19 & 2 & 14 & -8 & 37 & 35 & \\
\hline 11 & -8 & 38 & 49 & 55 & 8 & 14 & -8 & -37 & 71 & \\
\hline 15 & -8 & 80 & 59 & 18 & 12 & 14 & -8 & -29 & 105 & \\
\hline 10 & -8 & 26 & 71 & 61 & 1 & 15 & -8 & 39 & 120 & \\
\hline 16 & -8 & -45 & 47 & 60 & 3 & 15 & -8 & 47 & 71 & \\
\hline 7 & -8 & 73 & 79 & 14 & 5 & 15 & -8 & -91 & 11 & \\
\hline 9 & -8 & 72 & 84 & 16 & 7 & 15 & -8 & 90 & 77 & \\
\hline 15 & -8 & -145 & 31 & 30 & 11 & 15 & -8 & -35 & 39 & \\
\hline 2 & -8 & 60 & 17 & 15 & 13 & 15 & -8 & 71 & 69 & \\
\hline 10 & -8 & 64 & 95 & 47 & 2 & 16 & -8 & -71 & 150 & \\
\hline 12 & -8 & 69 & 47 & 50 & 4 & 16 & -8 & 39 & 37 & \\
\hline 16 & -8 & 0 & 74 & 95 & 12 & 16 & -8 & 32 & 47 & \\
\hline 5 & -8 & -96 & 29 & 25 & 3 & 17 & -8 & -27 & 37 & \\
\hline 11 & -2 & 55 & 22 & 51 & 11 & 17 & -8 & 0 & 34 & \\
\hline 12 & -8 & -91 & 47 & 42 & 2 & 18 & -8 & 77 & $6 \hat{9}$ & \\
\hline 14 & -8 & -89 & 61 & 44 & 4 & 18 & -8 & 55 & 10 & \\
\hline 16 & -8 & 0 & 73 & 92 & 12 & 18 & -8 & 42 & 54 & \\
\hline 1 & -8 & -64 & 75 & 34 & 5 & 19 & -8 & 73 & 9 & \\
\hline 5 & -8 & 61 & 63 & 42 & 9 & 19 & -8 & 0 & 3 & \\
\hline 13 & -8 & -64 & 34 & 43 & 11 & 19 & -8 & -26 & 83 & \\
\hline 2 & -8 & 62 & 16 & 17 & 6 & 20 & -8 & 43 & 87 & \\
\hline 8 & -8 & 66 & 153 & 43 & 10 & 20 & -8 & 37 & 50 & \\
\hline 12 & -8 & -39 & 10 & 61 & 12 & 20 & -8 & -100 & $i$ & \\
\hline 16 & -8 & 51 & 71 & 27 & 1 & 21 & -8 & 64 & 111 & \\
\hline 9 & -8 & 26 & 38 & 68 & 9 & 21 & -8 & -54 & $\leq$ & \\
\hline 13 & -8 & 53 & 55 & 50 & 11 & 21 & -8 & -32 & 31 & \\
\hline 15 & -8 & 48 & 102 & 60 & 2 & 22 & -8 & -19 & 49 & \\
\hline 810 & -8 & 57 & 51 & 50 & 6 & 22 & -8 & 78 & $13 \hat{L}$ & \\
\hline 1010 & -8 & -64 & 27 & 49 & 8 & 22 & -8 & 64 & 3 & \\
\hline 1210 & -8 & -58 & 17 & 21 & 10 & 22 & -8 & -93 & $\Sigma$ & \\
\hline 1410 & -8 & -87 & 134 & 48 & 1 & 23 & -8 & -71 & 42 & \\
\hline 11 & -8 & -55 & 31 & 43 & 3 & 23 & -8 & -126 & 62 & \\
\hline
\end{tabular}


Reflexões observadas $I \geq 3 \sigma$ (I)

Values of $10 *$ Fobs and $10^{*} \mathrm{FCalC}$

Page 11

\begin{tabular}{|c|c|c|c|c|c|c|c|c|c|c|c|}
\hline $\mathrm{H}$ & K & L & Fobs & Ecalc & SigF & $\mathrm{H}$ & K & I & Fobs & Ecalc & $\operatorname{Sig} E$ \\
\hline - & - & - & ---- & ----- & ---- & - & - & - & ---- & ----- & ---- \\
\hline 52 & 27 & -2 & 124 & 107 & 13 & 12 & 8 & -1 & 260 & 241 & 8 \\
\hline 3 & 1 & -1 & 825 & 835 & 7 & 1 & 9 & -1 & 334 & 326 & 5 \\
\hline 7 & 1 & -1 & 446 & 422 & 6 & 3 & 9 & -1 & 405 & 422 & 6 \\
\hline 9 & 1 & -1 & 208 & 195 & 6 & 5 & 9 & -1 & 290 & 295 & 4 \\
\hline 11 & 1 & -1 & 423 & 425 & 7 & 7 & 9 & -1 & 314 & 307 & 5 \\
\hline 15 & 1 & -1 & 150 & 130 & 12 & 9 & 9 & -1 & 136 & 146 & 9 \\
\hline 2 & 2 & -1 & 822 & 841 & 6 & 11 & 9 & -1 & 344 & 360 & 8 \\
\hline 4 & 2 & -1 & 267 & 265 & 4 & 13 & 9 & -1 & 183 & 189 & 10 \\
\hline 6 & 2 & -1 & 564 & 531 & 7 & 2 & 10 & -1 & 736 & 715 & 10 \\
\hline 8 & 2 & -1 & 551 & 559 & 9 & 6 & 10 & -1 & 283 & 259 & 5 \\
\hline 10 & 2 & -1 & 226 & 237 & 7 & 8 & 10 & -1 & 176 & 182 & 7 \\
\hline 1 & 3 & -1 & 1374 & 1416 & 5 & 10 & 10 & -1 & 193 & 202 & 9 \\
\hline 3 & 3 & -1 & 1196 & 1239 & 7 & 12 & 10 & -1 & 140 & 146 & 10 \\
\hline 5 & 3 & -1 & 1316 & 1249 & 9 & 14 & 10 & -1 & 107 & 91 & 16 \\
\hline 7 & 3 & -1 & 512 & 499 & 8 & 1 & 11 & -1 & 1642 & 1614 & 10 \\
\hline 9 & 3 & -1 & 143 & 121 & 8 & 5 & 11 & -1 & 294 & 302 & 5 \\
\hline 13 & 3 & -1 & 130 & 118 & 11 & 9 & 11 & -1 & 152 & 164 & 9 \\
\hline 15 & 3 & -1 & 229 & 227 & 10 & 11 & 11 & -1 & 348 & 358 & 7 \\
\hline 4 & 4 & -1 & 679 & 668 & 9 & 13 & 11 & -1 & 141 & 120 & 11 \\
\hline 6 & 4 & -1 & 1528 & 1482 & 10 & 2 & 12 & -1 & 516 & 474 & 8 \\
\hline 8 & 4 & -1 & 352 & 323 & 6 & 4 & 12 & -1 & 172 & 177 & 6 \\
\hline 10 & 4 & -1 & 336 & 362 & 6 & 6 & 12 & -1 & 236 & 228 & 6 \\
\hline 12 & 4 & -1 & 143 & 150 & 12 & 10 & 12 & -1 & 384 & 396 & 7 \\
\hline 1 & 5 & -1 & 366 & 380 & 6 & 1 & 13 & -1 & 565 & 514 & 8 \\
\hline 3 & 5 & -1 & 434 & 435 & 6 & 3 & 13 & -1 & 337 & 350 & 5 \\
\hline 5 & 5 & -1 & 654 & 611 & 9 & 5 & 13 & -1 & 264 & 231 & 5 \\
\hline 7 & 5 & -1 & 85 & 95 & 9 & 7 & 13 & -1 & 240 & 274 & 6 \\
\hline 9 & 5 & -1 & 356 & 355 & 5 & 2 & 14 & -1 & 176 & 197 & 6 \\
\hline 11 & 5 & -1 & 320 & 330 & 7 & 4 & 14 & -1 & 517 & 529 & 7 \\
\hline 2 & 6 & -1 & 495 & 512 & 6 & 6 & 14 & -1 & 337 & 361 & 6 \\
\hline 6 & 6 & -1 & 1016 & 962 & 11 & 10 & 14 & -1 & 328 & 368 & 7 \\
\hline 8 & 6 & -1 & 522 & 543 & 8 & 1 & 15 & -1 & 227 & 277 & 5 \\
\hline 10 & 6 & -1 & 115 & 137 & 10 & 3 & 15 & -1 & 642 & 627 & 9 \\
\hline 12 & 6 & -1 & 132 & 145 & 11 & 5 & 15 & -1 & 290 & 282 & 5 \\
\hline 1 & 7 & -1 & 230 & 240 & 4 & 7 & 15 & -1 & 446 & 458 & 6 \\
\hline 3 & 7 & -1 & 100 & 118 & 6 & 9 & 15 & -1 & 244 & 245 & 8 \\
\hline 5 & 7 & -1 & 1201 & 1162 & 10 & 13 & 15 & -1 & 196 & 196 & 11 \\
\hline 9 & 7 & -1 & 167 & 154 & 8 & 2 & 16 & -1 & 272 & 272 & 6 \\
\hline 11 & 7 & -1 & 262 & 280 & 8 & 4 & 16 & -1 & 119 & 135 & 9 \\
\hline 13 & 7 & -1 & 147 & 169 & 11 & 6 & 16 & -1 & 490 & 507 & 8 \\
\hline 2 & 8 & -1 & 1001 & 1019 & 9 & 8 & 16 & -1 & 614 & 631 & \\
\hline 4 & 8 & -1 & 1291 & 1240 & 9 & 1 & 17 & -1 & 376 & 322 & 0 \\
\hline 6 & 8 & -1 & 400 & 397 & 6 & 3 & 17 & -1 & 417 & 449 & 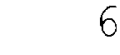 \\
\hline 8 & 8 & -1 & 351 & 357 & 5 & 7 & 17 & -1 & 201 & 180 & \\
\hline
\end{tabular}


Reflexões observadas $I \geq 3 \sigma$ (I)

Values of $10 *$ Fobs and $10 * \mathrm{Fcalc}$

Page 12

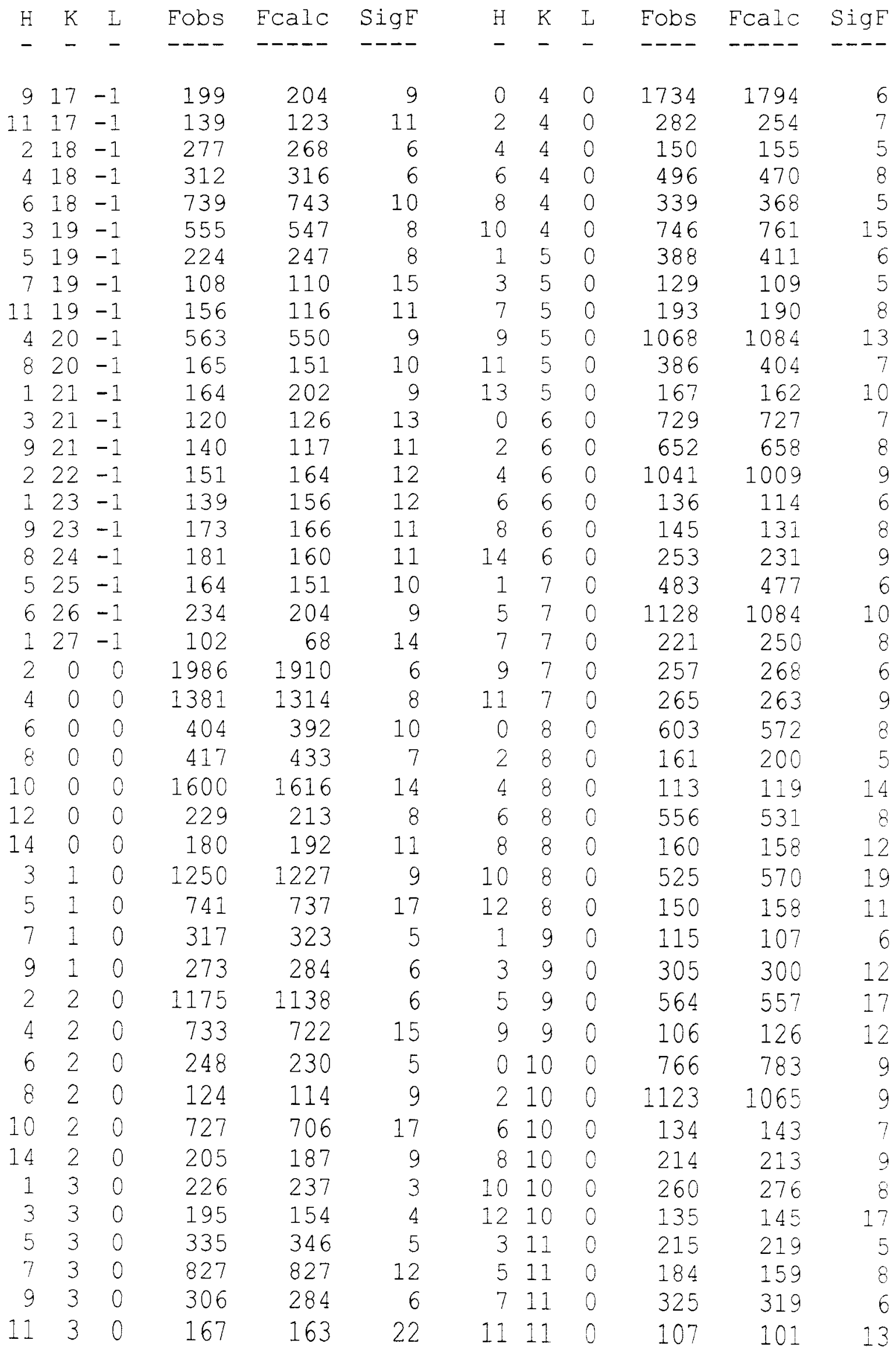


Reflexões observadas $I \geq 3 \sigma$ (I)

Values of $10 *$ Fobs and $10 * \mathrm{Fcalc}$

Page 13

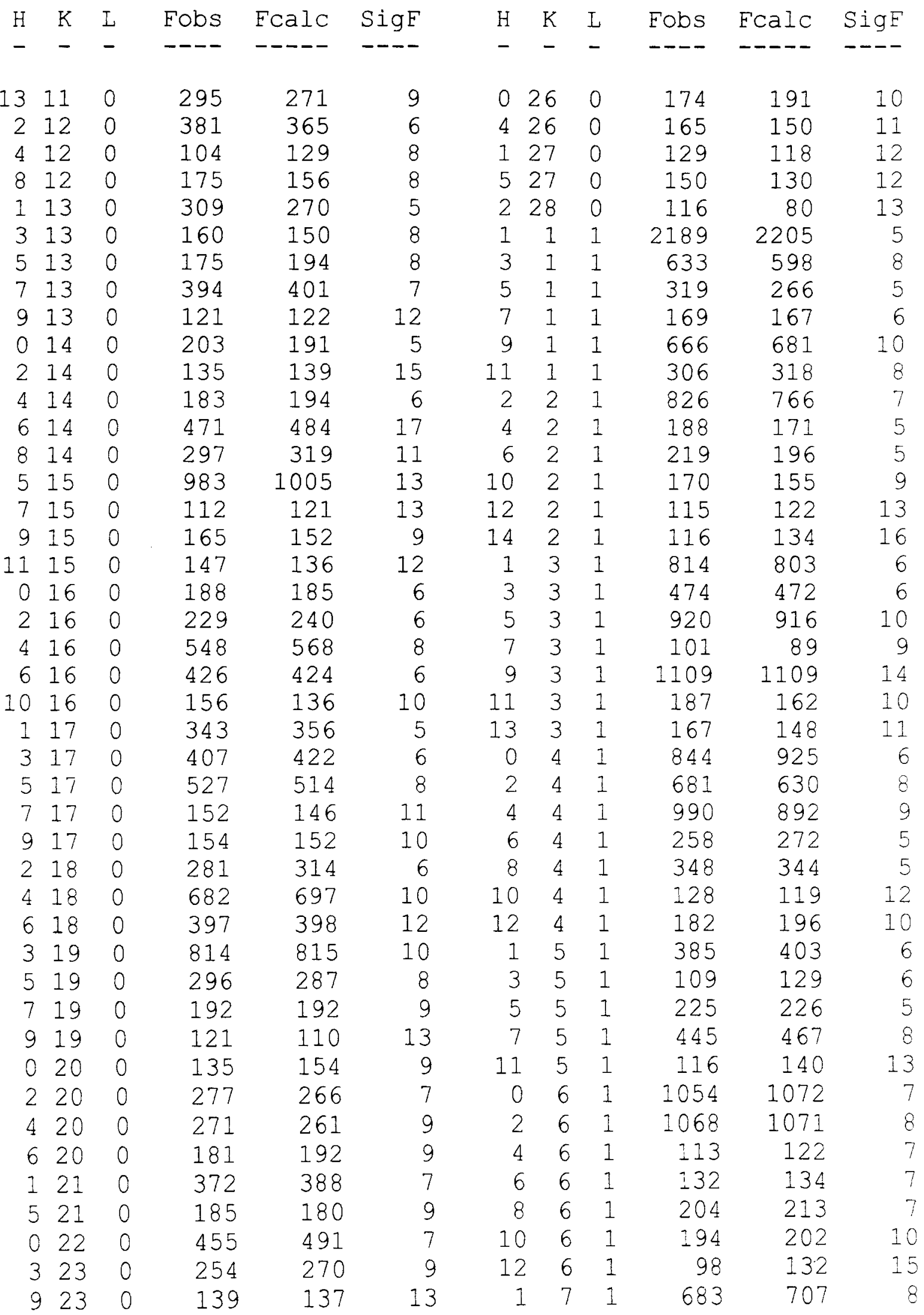


Reflexões observadas $I \geq 3 \sigma$ (I)

Values of $10 *$ Fobs and $10^{*} \mathrm{FCalc}$

Page 14

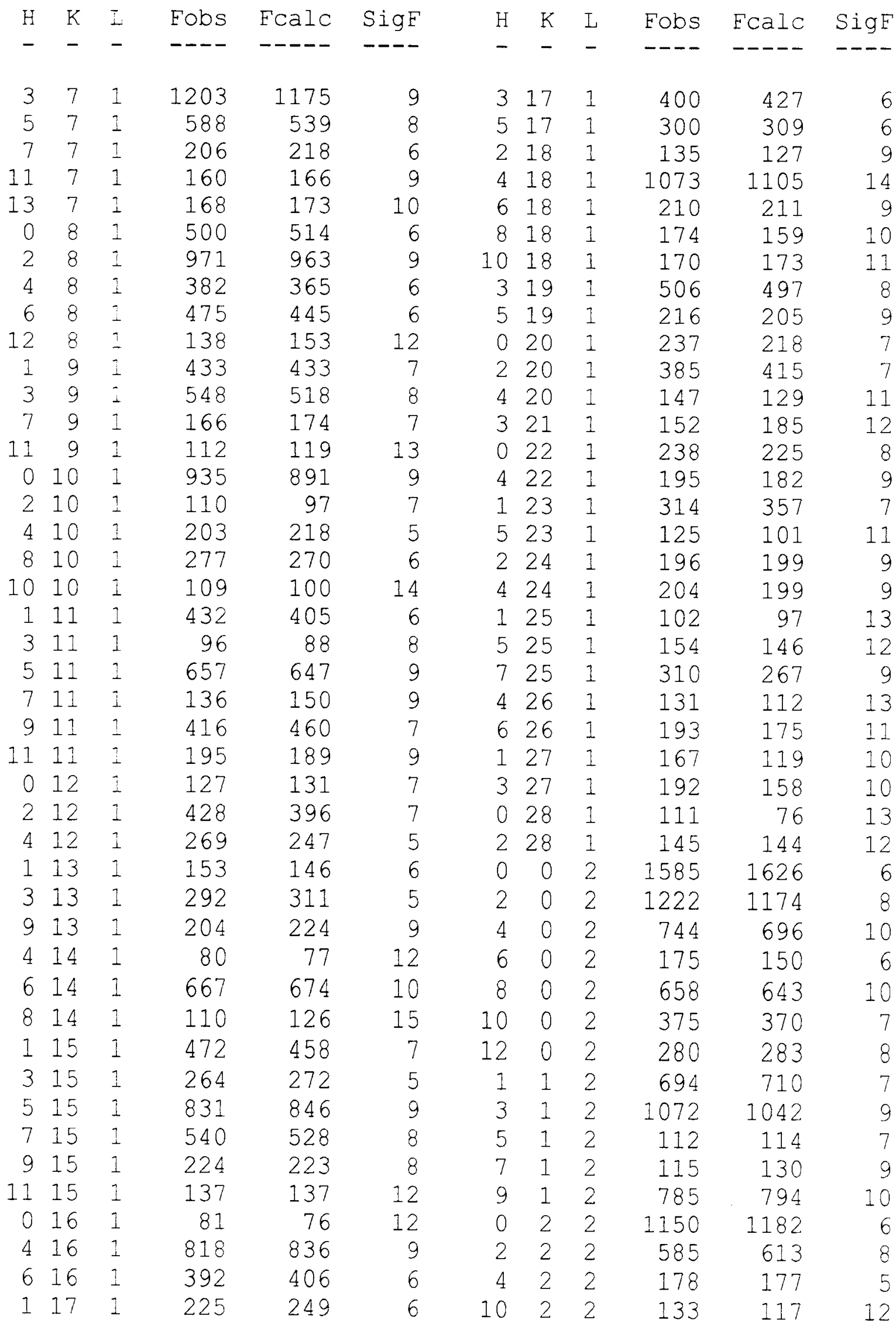


Reflexões observadas $I \geq 3 \sigma$ (I)

Values of $10^{*}$ Fobs and $10^{*} \mathrm{FcalC}$

Page 15

\begin{tabular}{|c|c|c|c|c|c|c|c|c|c|c|c|}
\hline $\mathrm{H}$ & K & I & Fobs & Fcalc & $\operatorname{Sig} F$ & $\mathrm{H}$ & K & L & Fobs & Fcalc & $\operatorname{Sig} F$ \\
\hline- & - & - & --- & ---- & - & - & - & - & ---- & ---- & - \\
\hline 12 & 2 & 2 & 234 & 219 & 9 & 6 & 12 & 2 & 232 & 231 & 7 \\
\hline 1 & 3 & 2 & 707 & 708 & 8 & 8 & 12 & 2 & 130 & 165 & 13 \\
\hline 3 & 3 & 2 & 416 & 387 & 7 & 10 & 12 & 2 & 205 & 220 & 9 \\
\hline 5 & 3 & 2 & 268 & 257 & 5 & 1 & 13 & 2 & 109 & 65 & 8 \\
\hline 7 & 3 & 2 & 474 & 453 & 7 & 5 & 13 & 2 & 305 & 320 & 6 \\
\hline 9 & 3 & 2 & 255 & 268 & 7 & 0 & 14 & 2 & 505 & 464 & 7 \\
\hline 0 & 4 & 2 & 598 & 639 & 8 & 2 & 14 & 2 & 331 & 337 & 0 \\
\hline 2 & 4 & 2 & 799 & 795 & 8 & 4 & 14 & 2 & 115 & 126 & 10 \\
\hline 4 & 4 & 2 & 619 & 566 & 7 & 6 & 14 & 2 & 662 & 692 & 10 \\
\hline 6 & 4 & 2 & 315 & 280 & 5 & 8 & 14 & 2 & 443 & 478 & 7 \\
\hline 8 & 4 & 2 & 544 & 580 & 7 & 3 & 15 & 2 & 327 & 333 & 5 \\
\hline 10 & 4 & 2 & 548 & 561 & 8 & 5 & 15 & 2 & 497 & 512 & 7 \\
\hline 1 & 5 & 2 & 194 & 189 & 4 & 9 & 15 & 2 & 164 & 149 & 10 \\
\hline 3 & 5 & 2 & 884 & 824 & 9 & 0 & 16 & 2 & 155 & 183 & 1 \\
\hline 5 & 5 & 2 & 1108 & 1078 & 11 & 2 & 16 & 2 & 198 & 200 & 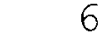 \\
\hline 7 & 5 & 2 & 97 & 79 & 10 & 4 & 16 & 2 & 287 & 268 & 6 \\
\hline 9 & 5 & 2 & 323 & 339 & 7 & 1 & 17 & 2 & 199 & 179 & \\
\hline 13 & 5 & 2 & 140 & 150 & 12 & 3 & 17 & 2 & 249 & 248 & \\
\hline 0 & 6 & 2 & 288 & 303 & 4 & 5 & 17 & 2 & 500 & 500 & \\
\hline 2 & 6 & 2 & 390 & 349 & 6 & 0 & 18 & 2 & 285 & 275 & \\
\hline 6 & 6 & 2 & 334 & 355 & 5 & 2 & 18 & 2 & 597 & 592 & \\
\hline 8 & 6 & 2 & 361 & 375 & 6 & 4 & 18 & 2 & 101 & 10 & \\
\hline 1 & 7 & 2 & 327 & 310 & 5 & 8 & 18 & 2 & 147 & 149 & 12 \\
\hline 3 & 7 & 2 & 1271 & 1183 & 10 & 1 & 19 & 2 & 617 & 649 & - \\
\hline 5 & 7 & 2 & 433 & 402 & 6 & 3 & 19 & 2 & 281 & 289 & \\
\hline 7 & 7 & 2 & 163 & 139 & 8 & 5 & 19 & 2 & 323 & 321 & \\
\hline 9 & 7 & 2 & 189 & 211 & 9 & 2 & 20 & 2 & 145 & 161 & 2 \\
\hline 11 & 7 & 2 & 178 & 192 & 10 & 1 & 21 & 2 & 206 & 212 & \\
\hline 13 & 7 & 2 & 215 & 196 & 9 & 3 & 21 & 2 & 145 & 148 & \\
\hline 2 & 8 & 2 & 430 & 456 & 8 & 5 & 21 & 2 & 110 & 116 & \\
\hline 4 & 8 & 2 & 446 & 446 & 6 & 0 & 22 & 2 & 286 & 324 & \\
\hline 6 & 8 & 2 & 313 & 304 & 5 & 1 & 23 & 2 & 302 & 315 & \\
\hline 10 & 8 & 2 & 271 & 290 & 7 & 5 & 25 & 2 & 105 & 83 & \\
\hline 1 & 9 & 2 & 201 & 160 & 5 & 2 & 26 & 2 & 117 & 115 & \\
\hline 3 & 9 & 2 & 599 & 626 & 8 & 3 & 27 & 2 & 134 & 111 & \\
\hline 5 & 9 & 2 & 258 & 250 & 5 & 2 & 28 & 2 & 117 & 72 & \\
\hline 7 & 9 & 2 & 226 & 228 & 7 & 1 & 1 & 3 & 173 & 188 & \\
\hline 0 & 10 & 2 & 194 & 254 & 5 & 3 & 1 & 3 & 201 & 203 & \\
\hline 6 & 10 & 2 & 106 & 121 & 10 & 5 & 1 & 3 & 301 & 302 & \\
\hline 1 & 11 & 2 & 692 & 664 & 8 & 7 & 1 & 3 & 343 & 358 & \\
\hline 3 & 11 & 2 & 69 & 51 & 11 & 9 & 1 & 3 & 454 & 480 & \\
\hline 7 & 11 & 2 & 395 & 393 & 6 & 13 & 1 & 3 & 187 & 171 & \\
\hline 0 & 12 & 2 & 1172 & 1144 & 10 & 0 & 2 & 3 & 901 & 899 & \\
\hline 2 & 12 & 2 & 813 & 819 & 11 & 2 & 2 & 3 & 99 & 116 & \\
\hline
\end{tabular}


Reflexões observadas $I \geq 3 \sigma$ (I)

Values of $10 *$ Fobs and $10 *$ Fcalc

Page 16

\begin{tabular}{|c|c|c|c|c|c|c|c|c|c|c|c|}
\hline $\mathrm{H}$ & K & I & Eobs & Fcalc & SigF & $\mathrm{H}$ & $\mathrm{K}$ & $\mathrm{L}$ & Fobs & Fcalc & $\operatorname{SigF}$ \\
\hline - & - & - & ---- & ----- & ---- & - & - & - & ---- & ----- & ---- \\
\hline 4 & 2 & 3 & 167 & 148 & 6 & 8 & 10 & 3 & 247 & 251 & 8 \\
\hline 6 & 2 & 3 & 362 & 361 & 5 & 10 & 10 & 3 & 106 & 119 & 14 \\
\hline 8 & 2 & 3 & 299 & 300 & 6 & 1 & 11 & 3 & 208 & 217 & 5 \\
\hline 10 & 2 & 3 & 200 & 206 & 8 & 3 & 11 & 3 & 231 & 264 & 5 \\
\hline 1 & 3 & 3 & 272 & 263 & 4 & 5 & 11 & 3 & 117 & 105 & 9 \\
\hline 3 & 3 & 3 & 1000 & 990 & 10 & 7 & 11 & 3 & 226 & 230 & 8 \\
\hline 5 & 3 & 3 & 326 & 329 & 5 & 9 & 11 & 3 & 355 & 387 & 7 \\
\hline 7 & 3 & 3 & 119 & 134 & 10 & 0 & 12 & 3 & 313 & 320 & 4 \\
\hline 9 & 3 & 3 & 206 & 212 & 10 & 2 & 12 & 3 & 114 & 113 & 8 \\
\hline 13 & 3 & 3 & 182 & 174 & 11 & 4 & 12 & 3 & 220 & 212 & 6 \\
\hline 0 & 4 & 3 & 240 & 244 & 4 & 6 & 12 & 3 & 103 & 102 & 12 \\
\hline 2 & 4 & 3 & 368 & 375 & 6 & 8 & 12 & 3 & 368 & 403 & 8 \\
\hline 4 & 4 & 3 & 976 & 955 & 11 & 1 & 13 & 3 & 410 & 393 & 6 \\
\hline 6 & 4 & 3 & 164 & 117 & 7 & 3 & 13 & 3 & 155 & 172 & 8 \\
\hline 8 & 4 & 3 & 371 & 387 & 6 & 5 & 13 & 3 & 169 & 177 & 8 \\
\hline 1 & 5 & 3 & 502 & 524 & 7 & 2 & 14 & 3 & 400 & 402 & 7 \\
\hline 3 & 5 & 3 & 925 & 854 & 10 & 4 & 14 & 3 & 195 & 223 & 7 \\
\hline 7 & 5 & 3 & 367 & 370 & 6 & 8 & 14 & 3 & 256 & 297 & 8 \\
\hline 9 & 5 & 3 & 151 & 171 & 12 & 1 & 15 & 3 & 405 & 462 & 6 \\
\hline 0 & 6 & 3 & 455 & 470 & 7 & 3 & 15 & 3 & 128 & 138 & 9 \\
\hline 2 & 6 & 3 & 307 & 314 & 4 & 5 & 15 & 3 & 486 & 489 & 7 \\
\hline 4 & 6 & 3 & 1290 & 1208 & 11 & 0 & 16 & 3 & 345 & 362 & 5 \\
\hline 6 & 6 & 3 & 363 & 368 & 6 & 4 & 16 & 3 & 431 & 429 & 7 \\
\hline 8 & 6 & 3 & 160 & 164 & 10 & 6 & 16 & 3 & 532 & 560 & 7 \\
\hline 10 & 6 & 3 & 166 & 175 & 10 & 8 & 16 & 3 & 112 & 105 & 13 \\
\hline 1 & 7 & 3 & 224 & 257 & 5 & 1 & 17 & 3 & 489 & 471 & 6 \\
\hline 3 & 7 & 3 & 870 & 865 & 11 & 5 & 17 & 3 & 130 & 134 & 14 \\
\hline 7 & 7 & 3 & 108 & 94 & 11 & 7 & 17 & 3 & 142 & 160 & 11 \\
\hline 9 & 7 & 3 & 205 & 214 & 10 & 0 & 18 & 3 & 162 & 154 & 8 \\
\hline 11 & 7 & 3 & 175 & 164 & 10 & 2 & 18 & 3 & 416 & 420 & 6 \\
\hline 0 & 8 & 3 & 805 & 765 & 9 & 4 & 18 & 3 & 665 & 670 & 9 \\
\hline 2 & 8 & 3 & 1314 & 1310 & 10 & 1 & 19 & 3 & 344 & 379 & 7 \\
\hline 4 & 8 & 3 & 561 & 555 & 9 & 3 & 19 & 3 & 165 & 198 & 11 \\
\hline 6 & 8 & 3 & 270 & 270 & 6 & 2 & 20 & 3 & 385 & 391 & 7 \\
\hline 10 & 8 & 3 & 187 & 197 & 10 & 6 & 20 & 3 & 188 & 176 & 10 \\
\hline 1 & 9 & 3 & 610 & 641 & 8 & 1 & 21 & 3 & 122 & 102 & 13 \\
\hline 3 & 9 & 3 & 222 & 188 & 5 & 0 & 22 & 3 & 123 & 123 & 14 \\
\hline 5 & 9 & 3 & 315 & 298 & 5 & 4 & 22 & 3 & 107 & 103 & 14 \\
\hline 7 & 9 & 3 & 139 & 150 & 9 & 6 & 22 & 3 & 97 & 72 & 15 \\
\hline 9 & 9 & 3 & 337 & 340 & 8 & 7 & 23 & 3 & 182 & 159 & 10 \\
\hline 11 & 9 & 3 & 166 & 159 & 11 & 3 & 25 & 3 & 123 & 98 & 12 \\
\hline 0 & 10 & 3 & 657 & 705 & 7 & 4 & 26 & 3 & 247 & 207 & 9 \\
\hline 2 & 10 & 3 & 81 & 88 & 10 & 0 & 0 & 4 & 764 & 762 & 8 \\
\hline 6 & 10 & 3 & 227 & 240 & 7 & 2 & 0 & 4 & 607 & 638 & \\
\hline
\end{tabular}


Reflexões observadas $I \geq 3 \sigma$ (I)

Values of $10 *$ Fobs and $10 *$ Fcalc

Page 17

\begin{tabular}{|c|c|c|c|c|c|c|c|c|c|c|c|}
\hline $\mathrm{H}$ & K & $\mathrm{L}$ & Fobs & Ecalc & $\operatorname{Sig} E$ & $\mathrm{H}$ & K & $L$ & Fobs & Ecalc & SigE \\
\hline - & - & - & ---- & ----- & --- & - & - & - & ---- & --- & ---- \\
\hline 4 & 0 & 4 & 571 & 584 & 8 & 6 & 10 & 4 & 98 & 75 & 11 \\
\hline 6 & 0 & 4 & 126 & 130 & 10 & 8 & 10 & 4 & 247 & 259 & 9 \\
\hline 8 & 0 & 4 & 1285 & 1288 & 14 & 10 & 10 & 4 & 195 & 188 & 9 \\
\hline 10 & 0 & 4 & 216 & 214 & 9 & 5 & 11 & 4 & 331 & 339 & 6 \\
\hline 1 & 1 & 4 & 506 & 502 & 7 & 11 & 11 & 4 & 275 & 246 & 8 \\
\hline 3 & 1 & 4 & 544 & 553 & 8 & 0 & 12 & 4 & 342 & 330 & 5 \\
\hline 5 & 1 & 4 & 302 & 321 & 5 & 4 & 12 & 4 & 147 & 174 & 9 \\
\hline 7 & 1 & 4 & 264 & 273 & 6 & 8 & 12 & 4 & 133 & 122 & 10 \\
\hline 0 & 2 & 4 & 421 & 433 & 7 & 3 & 13 & 4 & 196 & 181 & 7 \\
\hline 2 & 2 & 4 & 488 & 532 & 7 & 5 & 13 & 4 & 279 & 246 & 7 \\
\hline 6 & 2 & 4 & 341 & 349 & 5 & 7 & 13 & 4 & 129 & 170 & 15 \\
\hline 8 & 2 & 4 & 693 & 708 & 11 & 9 & 13 & 4 & 113 & 113 & 15 \\
\hline 10 & 2 & 4 & 102 & 91 & 14 & 0 & 14 & 4 & 128 & 104 & 8 \\
\hline 12 & 2 & 4 & 125 & 97 & 13 & 2 & 14 & 4 & 274 & 304 & 6 \\
\hline 1 & 3 & 4 & 400 & 419 & 6 & 4 & 14 & 4 & 191 & 195 & 8 \\
\hline 5 & 3 & 4 & 567 & 556 & 9 & 6 & 14 & 4 & 208 & 205 & 9 \\
\hline 7 & 3 & 4 & 381 & 375 & 6 & 1 & 15 & 4 & 120 & 157 & 10 \\
\hline 9 & 3 & 4 & 132 & 122 & 12 & 3 & 15 & 4 & 825 & 859 & 10 \\
\hline 0 & 4 & 4 & 77 & 84 & 9 & 0 & 16 & 4 & 202 & 210 & 7 \\
\hline 4 & 4 & 4 & 313 & 295 & 5 & 2 & 16 & 4 & 482 & 502 & 8 \\
\hline 8 & 4 & 4 & 534 & 532 & 8 & 4 & 16 & 4 & 465 & 464 & 7 \\
\hline 1 & 5 & 4 & 662 & 645 & 10 & 8 & 16 & 4 & 104 & 81 & 14 \\
\hline 3 & 5 & 4 & 391 & 372 & 6 & 1 & 17 & 4 & 248 & 252 & 7 \\
\hline 5 & 5 & 4 & 268 & 265 & 5 & 3 & 17 & 4 & 495 & 8 & 7 \\
\hline 7 & 5 & 4 & 604 & 632 & 9 & 5 & 17 & 4 & 175 & 172 & 11 \\
\hline 9 & 5 & 4 & 194 & 188 & 8 & 7 & 17 & 4 & 114 & 114 & 14 \\
\hline 11 & 5 & 4 & 138 & 135 & 12 & 0 & 18 & 4 & 202 & 231 & 7 \\
\hline 0 & 6 & 4 & 331 & 344 & 6 & 2 & 18 & 4 & 570 & 566 & 9 \\
\hline 2 & 6 & 4 & 876 & 866 & 11 & 4 & 18 & 4 & 356 & 353 & 7 \\
\hline 6 & 6 & 4 & 163 & 170 & 8 & 6 & 18 & 4 & 113 & 104 & 13 \\
\hline 12 & 6 & 4 & 237 & 227 & 10 & 1 & 19 & 4 & 620 & 619 & 11 \\
\hline 1 & 7 & 4 & 270 & 293 & 5 & 3 & 19 & 4 & 198 & 195 & 10 \\
\hline 3 & 7 & 4 & 627 & 619 & 8 & 5 & 19 & 4 & 173 & 167 & 10 \\
\hline 5 & 7 & 4 & 107 & 138 & 10 & 0 & 20 & 4 & 215 & 234 & 9 \\
\hline 9 & 7 & 4 & 302 & 292 & 7 & 2 & 20 & 4 & 167 & 152 & 11 \\
\hline 0 & 8 & 4 & 181 & 200 & 5 & 4 & 20 & 4 & 207 & 206 & 9 \\
\hline 4 & 8 & 4 & 355 & 341 & 5 & 1 & 23 & 4 & 127 & 160 & 13 \\
\hline 6 & 8 & 4 & 176 & 178 & 8 & 0 & 28 & 4 & 108 & 74 & 14 \\
\hline 8 & 8 & 4 & 389 & 413 & 7 & 1 & 1 & 5 & 447 & 436 & 6 \\
\hline 1 & 9 & 4 & 95 & 113 & 9 & 7 & 1 & 5 & 477 & 497 & 6 \\
\hline 3 & 9 & 4 & 284 & 293 & 5 & 9 & 2 & 5 & 169 & 165 & 10 \\
\hline 9 & 9 & 4 & 97 & 86 & 14 & 0 & 2 & 5 & 104 & 99 & \\
\hline 0 & 10 & 4 & 812 & 804 & 11 & 6 & 2 & 5 & 151 & 153 & \\
\hline 2 & 10 & 4 & 121 & 122 & 8 & 1 & 3 & 5 & 406 & 425 & \\
\hline
\end{tabular}


Reflexões observadas $I \geq 3 \sigma$ (I)

Values of $10 *$ Fobs and $10 *$ EcalC

Page 18

\begin{tabular}{|c|c|c|c|c|c|c|c|c|c|c|c|}
\hline $\mathrm{H}$ & K & L & Fobs & Fcalc & $\operatorname{SigF}$ & $\mathrm{H}$ & $\mathrm{K}$ & L & Eobs & Fcalc & SigE \\
\hline- & - & - & ---- & ----- & ---- & - & - & - & ---- & ----- & ---- \\
\hline 3 & 3 & 5 & 659 & 629 & 8 & 0 & 18 & 5 & 146 & 164 & 11 \\
\hline 7 & 3 & 5 & 810 & 806 & 11 & 2 & 18 & 5 & 815 & 817 & 11 \\
\hline 9 & 3 & 5 & 193 & 199 & 9 & 4 & 18 & 5 & 212 & 229 & 8 \\
\hline 2 & 4 & 5 & 542 & 552 & 8 & 1 & 19 & 5 & 296 & 321 & 8 \\
\hline 4 & 4 & 5 & 188 & 200 & 7 & 3 & 19 & 5 & 145 & 142 & 11 \\
\hline 6 & 4 & 5 & 141 & 136 & 9 & 0 & 20 & 5 & 245 & 234 & 8 \\
\hline 10 & 4 & 5 & 152 & 127 & 11 & 1 & 21 & 5 & 124 & 116 & 12 \\
\hline 1 & 5 & 5 & 96 & 100 & 9 & 2 & 22 & 5 & 139 & 116 & 11 \\
\hline 3 & 5 & 5 & 139 & 151 & 8 & 0 & 24 & 5 & 201 & 204 & 9 \\
\hline 5 & 5 & 5 & 268 & 275 & 6 & 2 & 24 & 5 & 185 & 181 & 11 \\
\hline 9 & 5 & 5 & 122 & 98 & 12 & 2 & 26 & 5 & 105 & 81 & 15 \\
\hline 0 & 6 & 5 & 165 & 182 & 6 & 1 & 27 & 5 & 141 & 122 & 13 \\
\hline 2 & 6 & 5 & 135 & 134 & 8 & 0 & 0 & 6 & 328 & 300 & 5 \\
\hline 4 & 6 & 5 & 276 & 268 & 6 & 2 & 0 & 6 & 379 & 375 & 5 \\
\hline 6 & 6 & 5 & 233 & 246 & 7 & 6 & 0 & 6 & 431 & 447 & 6 \\
\hline 1 & 7 & 5 & 569 & 552 & 8 & 8 & 0 & 6 & 293 & 323 & 8 \\
\hline 3 & 7 & 5 & 394 & 385 & 6 & 10 & 0 & 6 & 226 & 216 & 9 \\
\hline 5 & 7 & 5 & 106 & 107 & 11 & 1 & 1 & 6 & 496 & 497 & 7 \\
\hline 9 & 7 & 5 & 117 & 128 & 13 & 7 & 1 & 6 & 655 & 657 & 9 \\
\hline 11 & 7 & 5 & 152 & 119 & 11 & 0 & 2 & 6 & 471 & 480 & 6 \\
\hline 0 & 8 & 5 & 292 & 294 & 5 & 8 & 2 & 6 & 119 & 143 & 12 \\
\hline 2 & 8 & 5 & 187 & 176 & 6 & 10 & 2 & 6 & 152 & 136 & 12 \\
\hline 4 & 8 & 5 & 293 & 281 & 6 & 1 & 3 & 6 & 233 & 204 & 5 \\
\hline 1 & 9 & 5 & 201 & 169 & 6 & 3 & 3 & 6 & 82 & 96 & 12 \\
\hline 3 & 9 & 5 & 167 & 161 & 7 & 5 & 3 & 6 & 264 & 287 & 6 \\
\hline 0 & 10 & 5 & 91 & 91 & 10 & 0 & 4 & 6 & 336 & 319 & 5 \\
\hline 2 & 10 & 5 & 145 & 161 & 8 & 2 & 4 & 6 & 272 & 284 & 5 \\
\hline 1 & 11 & 5 & 132 & 123 & 8 & 4 & 4 & 6 & 166 & 180 & 8 \\
\hline 3 & 11 & 5 & 306 & 319 & 6 & 6 & 4 & 6 & 315 & 281 & 7 \\
\hline 7 & 11 & 5 & 278 & 300 & 7 & 8 & 4 & 6 & 349 & 324 & 7 \\
\hline 0 & 12 & 5 & 267 & 262 & 6 & 1 & 5 & 6 & 335 & 320 & 6 \\
\hline 2 & 12 & 5 & 139 & 149 & 9 & 3 & 5 & 6 & 496 & 480 & 7 \\
\hline 1 & 13 & 5 & 271 & 280 & 6 & 7 & 5 & 6 & 156 & 175 & 13 \\
\hline 7 & 13 & 5 & 138 & 167 & 12 & 0 & 6 & 6 & 152 & 159 & 7 \\
\hline 4 & 14 & 5 & 414 & 414 & 6 & 4 & 6 & 6 & 182 & 189 & 8 \\
\hline 1 & 15 & 5 & 244 & 213 & 6 & 6 & 6 & 6 & 204 & 179 & 9 \\
\hline 3 & 15 & 5 & 462 & 470 & 7 & 1 & 7 & 6 & 747 & 736 & 8 \\
\hline 5 & 15 & 5 & 426 & 419 & 7 & 3 & 7 & 6 & 378 & 371 & 6 \\
\hline 7 & 15 & 5 & 110 & 130 & 15 & 0 & 8 & 6 & 304 & 333 & 5 \\
\hline 9 & 15 & 5 & 143 & 136 & 13 & 2 & 8 & 6 & 221 & 220 & 6 \\
\hline 2 & 16 & 5 & 660 & 650 & 9 & 4 & 8 & 6 & 128 & 129 & 9 \\
\hline 4 & 16 & 5 & 229 & 214 & 10 & 8 & 8 & 6 & 199 & 192 & 9 \\
\hline 1 & 17 & 5 & 518 & 516 & 8 & 1 & 9 & 6 & 353 & 345 & 5 \\
\hline 3 & 17 & 5 & 398 & 371 & 8 & 3 & 9 & 6 & 129 & 122 & 10 \\
\hline
\end{tabular}


Reflexões observadas $I \geq 3 \sigma$ (I)

Values of $10 *$ Fobs and $10 *$ Fcalc

Page 19

\begin{tabular}{|c|c|c|c|c|c|c|c|c|c|c|}
\hline K & $\mathrm{L}$ & Fobs & Ecalc & $\operatorname{SigF}$ & $\mathrm{H}$ & $\mathrm{K}$ & I & Fobs & Fcalc & SigE \\
\hline - & - & ---- & ----- & ---- & - & - & - & --- & $-\cdots$ & $\cdots-$ \\
\hline 9 & 6 & 143 & 153 & 11 & 5 & 9 & 7 & 145 & 134 & 13 \\
\hline 410 & 6 & 107 & 85 & 11 & 7 & 9 & 7 & 235 & 252 & 9 \\
\hline 511 & 6 & 218 & 231 & 10 & 6 & 10 & 7 & 187 & 203 & 10 \\
\hline 012 & 6 & 323 & 313 & 5 & 1 & 11 & 7 & 129 & 128 & 10 \\
\hline 412 & 6 & 113 & 89 & 13 & 7 & 11 & 7 & 273 & 272 & 8 \\
\hline 612 & 6 & 141 & 161 & 11 & 6 & 12 & 7 & 285 & 282 & 8 \\
\hline 812 & 6 & 156 & 160 & 11 & 3 & 13 & 7 & 138 & 135 & 13 \\
\hline 313 & 6 & 169 & 149 & 10 & 0 & 14 & 7 & 234 & 222 & 7 \\
\hline 414 & 6 & 402 & 376 & 7 & 4 & 14 & 7 & 114 & 128 & 13 \\
\hline 614 & 6 & 298 & 313 & 8 & 6 & 14 & 7 & 149 & 165 & 12 \\
\hline 115 & 6 & 196 & 190 & 8 & 3 & 15 & 7 & 317 & 304 & 9 \\
\hline 315 & 6 & 278 & 264 & 9 & 2 & 16 & 7 & 292 & 308 & 9 \\
\hline 715 & 6 & 110 & 87 & 14 & 4 & 16 & 7 & 330 & 305 & 8 \\
\hline 016 & 6 & 93 & 103 & 12 & 0 & 18 & 7 & 302 & 300 & 9 \\
\hline 216 & 6 & 260 & 283 & 8 & 2 & 18 & 7 & 368 & 368 & 7 \\
\hline 117 & 6 & 168 & 184 & 11 & 0 & 20 & 7 & 226 & 217 & 9 \\
\hline 317 & 6 & 324 & 344 & 8 & 2 & 0 & 8 & 241 & 243 & 7 \\
\hline 018 & 6 & 380 & 385 & 7 & 4 & 0 & 8 & 129 & 109 & 12 \\
\hline 218 & 6 & 146 & 149 & 13 & 6 & 0 & 8 & 775 & 773 & 12 \\
\hline 119 & 6 & 189 & 189 & 11 & 1 & 1 & 8 & 161 & 193 & 8 \\
\hline 319 & 6 & 226 & 214 & 8 & 3 & 1 & 8 & 153 & 159 & 9 \\
\hline 020 & 6 & 117 & 108 & 12 & 5 & 1 & 8 & 268 & 253 & 8 \\
\hline 1 & 7 & 177 & 143 & 8 & 7 & 1 & 8 & 119 & 130 & 13 \\
\hline 1 & 7 & 221 & 213 & 9 & 0 & 2 & 8 & 110 & 160 & 10 \\
\hline 2 & 7 & 186 & 179 & 7 & 2 & 2 & 8 & 93 & 76 & 12 \\
\hline 62 & 7 & 216 & 200 & 10 & 4 & 2 & 8 & 171 & 183 & 10 \\
\hline 2 & 7 & 151 & 134 & 11 & 6 & 2 & 8 & 439 & 479 & 7 \\
\hline 3 & 7 & 360 & 332 & 6 & 1 & 3 & 8 & 165 & 145 & 8 \\
\hline 3 & 7 & 159 & 140 & 8 & 3 & 3 & 8 & 222 & 211 & 7 \\
\hline 3 & 7 & 107 & 99 & 13 & 5 & 3 & 8 & 245 & 258 & 9 \\
\hline 4 & 7 & 220 & 214 & 6 & 7 & 3 & 8 & 144 & 134 & 11 \\
\hline 4 & 7 & 543 & 518 & 8 & 0 & 4 & 8 & 166 & 145 & 8 \\
\hline 4 & 7 & 202 & 233 & 10 & 2 & 4 & 8 & 145 & 158 & 9 \\
\hline 5 & 7 & 630 & 679 & 9 & 6 & 4 & 8 & 350 & 298 & 7 \\
\hline 5 & 7 & 187 & 171 & 10 & 1 & 5 & 8 & 230 & 240 & 7 \\
\hline 2 & 7 & 643 & 628 & 9 & 3 & 5 & 8 & 200 & 197 & 8 \\
\hline 6 & 7 & 182 & 178 & 8 & 5 & 5 & 8 & 298 & 291 & 9 \\
\hline 2 & 7 & 443 & 427 & 6 & 7 & 5 & 8 & 109 & 87 & 14 \\
\hline 7 & 7 & 136 & 119 & 12 & 0 & 6 & 8 & 417 & 417 & 7 \\
\hline 8 & 7 & 536 & 520 & 8 & 1 & 7 & 8 & 355 & 329 & 6 \\
\hline 8 & 7 & 172 & 187 & 9 & 7 & 7 & 8 & 148 & 139 & 12 \\
\hline 8 & 7 & 143 & 153 & 12 & 2 & 8 & 8 & 205 & 182 & 7 \\
\hline 8 & 7 & 136 & 134 & 13 & 6 & 8 & 8 & 222 & 197 & 10 \\
\hline 39 & 7 & 89 & 93 & 13 & 1 & 9 & 8 & 111 & 95 & 12 \\
\hline
\end{tabular}


Reflexões observadas $I \geq 3 \sigma$ (I)

Values of $10 *$ Eobs and $10 * \mathrm{Fcalc}$

Page 20

\begin{tabular}{|c|c|c|c|c|c|c|c|c|c|c|}
\hline $\mathrm{K}$ & $\mathrm{I}$ & Eobs & Ecalc & $\operatorname{Sig} F$ & $\mathrm{H}$ & K & I & Eobs & Fcalc & SigF \\
\hline- & - & ---- & $-\cdots$ & --- & - & - & - & --- & ---- & ---- \\
\hline 610 & 8 & 178 & 180 & 10 & 6 & 4 & 10 & 142 & 140 & 14 \\
\hline 311 & 8 & 174 & 192 & 12 & 1 & 5 & 10 & 157 & 134 & 11 \\
\hline 113 & 8 & 196 & 195 & 10 & 4 & 6 & 10 & 122 & 58 & 12 \\
\hline 313 & 8 & 158 & 137 & 10 & 1 & 7 & 10 & 160 & 191 & 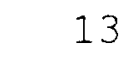 \\
\hline 713 & 8 & 110 & 90 & 15 & 3 & 9 & 10 & 115 & 93 & 13 \\
\hline 014 & 8 & 231 & 222 & 9 & 4 & 12 & 10 & 128 & 111 & 13 \\
\hline 414 & 8 & 110 & 118 & 15 & 2 & 14 & 10 & 131 & 126 & 15 \\
\hline 115 & 8 & 552 & 520 & 9 & 4 & 14 & 10 & 152 & 149 & 10 \\
\hline 016 & 8 & 356 & 359 & 8 & 1 & 15 & 10 & 163 & 124 & 11 \\
\hline 216 & 8 & 290 & 328 & 8 & 0 & 16 & 10 & 189 & 172 & 11 \\
\hline 117 & 8 & 362 & 380 & 7 & 1 & 17 & 10 & 153 & 146 & 14 \\
\hline 317 & 8 & 114 & 112 & 15 & 0 & 4 & 11 & 188 & 213 & 11 \\
\hline 018 & 8 & 300 & 302 & 8 & 0 & 6 & 11 & 280 & 294 & \\
\hline 218 & 8 & 236 & 239 & 9 & 4 & 12 & 11 & 171 & 148 & $\perp$ \\
\hline 319 & 8 & 160 & 130 & 11 & 1 & 13 & 11 & 104 & 80 & 10 \\
\hline 220 & 8 & 149 & 146 & 13 & 1 & 15 & 11 & 142 & 125 & 1 \\
\hline $\begin{array}{ll}5 & 1\end{array}$ & 9 & 235 & 261 & 9 & 0 & 16 & 11 & 166 & 145 & 1 \\
\hline 1 & 9 & 120 & 95 & 13 & 2 & 16 & 11 & 189 & 141 & 12 \\
\hline 2 & 9 & 170 & 179 & 11 & 0 & 18 & 11 & 176 & 170 & 1 \\
\hline 3 & 9 & 229 & 202 & 7 & 4 & 0 & 12 & 354 & 350 & \\
\hline 3 & 9 & 445 & 414 & 7 & 3 & 1 & 12 & 176 & 169 & 1. \\
\hline 3 & 9 & 137 & 134 & 13 & 4 & 2 & 12 & 247 & 282 & 1 \\
\hline 4 & 9 & 317 & 290 & 6 & 3 & 3 & 12 & 140 & 140 & 1 \\
\hline 4 & 9 & 119 & 120 & 15 & 4 & 4 & 12 & 153 & 130 & 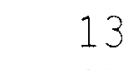 \\
\hline 6 & 9 & 184 & 183 & 10 & 3 & 5 & 12 & 111 & 118 & 1 \\
\hline 6 & 9 & 106 & 116 & 14 & 0 & 16 & 12 & 164 & 165 & 1 \\
\hline 7 & 9 & 247 & 227 & 7 & 3 & 3 & 13 & 173 & 154 & 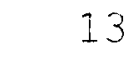 \\
\hline 7 & 9 & 110 & 61 & 16 & & & & & & \\
\hline 8 & 9 & 125 & 107 & 11 & & & & & & \\
\hline 8 & 9 & 147 & 126 & 12 & & & & & & \\
\hline 111 & 9 & 124 & 119 & 14 & & & & & & \\
\hline 511 & 9 & 127 & 129 & 15 & & & & & & \\
\hline 214 & 9 & 186 & 167 & 10 & & & & & & \\
\hline 115 & 9 & 174 & 173 & 11 & & & & & & \\
\hline 315 & 9 & 236 & 241 & 10 & & & & & & \\
\hline 016 & 9 & 385 & 373 & 7 & & & & & & \\
\hline 117 & 9 & 247 & 252 & 10 & & & & & & \\
\hline 018 & 9 & 436 & 414 & 7 & & & & & & \\
\hline 218 & 9 & 130 & 173 & 15 & & & & & & \\
\hline 40 & 10 & 146 & 159 & 12 & & & & & & \\
\hline 60 & 10 & 156 & 174 & 12 & & & & & & \\
\hline 1 & 10 & 379 & 377 & 8 & & & & & & \\
\hline 33 & 10 & 129 & 140 & 12 & & & & & & \\
\hline 44 & 10 & 140 & 87 & 12 & & & & & & \\
\hline
\end{tabular}


Reflexões não observadas $I<3 \sigma$ (I)

Values of $10^{*}$ Eobs and $10^{*}$ Ecalc

Page 1

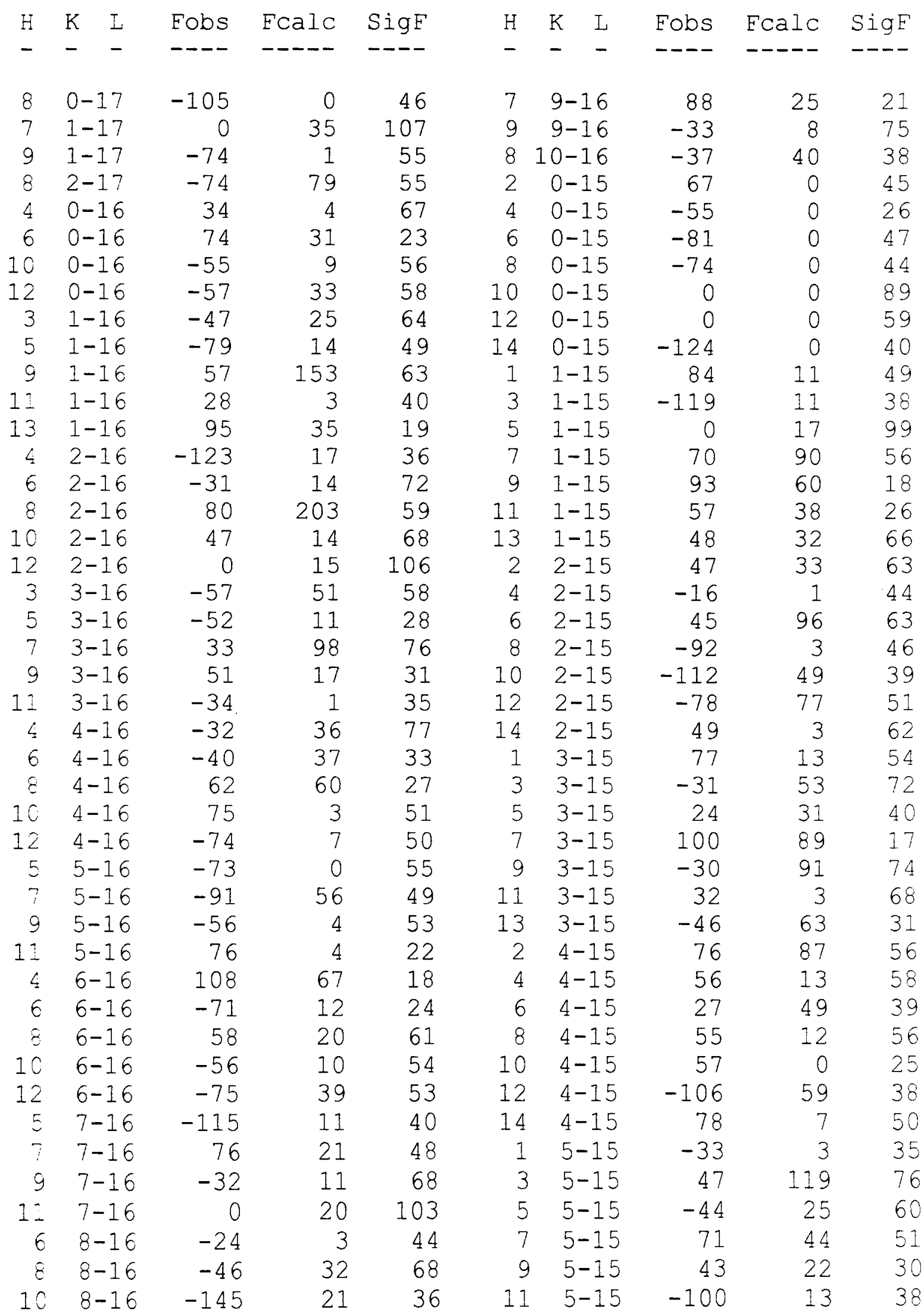


Reflexões não observadas $I<3 \sigma(I)$

Values of $10{ }^{*}$ Fobs and $10 * \mathrm{Fcalc}$

Page 2

\begin{tabular}{|c|c|c|c|c|c|c|c|c|c|}
\hline $\mathrm{H}$ & I & Fobs & Fcalc & SigF & $\mathrm{H}$ & L & Fobs & Ecalc & SigF \\
\hline- & - & --- & $-\cdots-$ & -- & - & - & ---- & -- & -- \\
\hline & $5-15$ & 0 & 33 & 92 & 12 & $0-14$ & -97 & 4 & 36 \\
\hline Z & $6-15$ & 59 & 104 & 64 & 14 & $0-14$ & -20 & 16 & 41 \\
\hline 4 & $6-15$ & 53 & 20 & 28 & 1 & $1-14$ & -54 & 56 & 53 \\
\hline 6 & $6-15$ & 52 & 48 & 28 & 3 & $1-14$ & 60 & 4 & 22 \\
\hline 8 & $6-15$ & -98 & 15 & 47 & 5 & $1-14$ & -117 & 83 & 35 \\
\hline 8 & $6-15$ & -104 & 7 & 39 & 7 & $1-14$ & -105 & 28 & 34 \\
\hline & $6-15$ & -112 & 17 & 41 & 9 & $1-14$ & 80 & 22 & 18 \\
\hline 3 & $7-15$ & -64 & 70 & 55 & 11 & $1-14$ & 0 & 47 & 92 \\
\hline 5 & $7-15$ & -63 & 6 & 52 & 13 & $1-14$ & -83 & 43 & 44 \\
\hline 7 & $7-15$ & 32 & 41 & 79 & 15 & $1-14$ & 35 & 4 & 7 \\
\hline 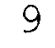 & $7-15$ & -62 & 9 & 49 & 2 & $2-14$ & -28 & 8 & 35 \\
\hline & $7-15$ & -111 & 28 & 36 & 4 & $2-14$ & 68 & 36 & 21 \\
\hline & $7-15$ & 49 & 5 & 62 & 6 & $2-14$ & -68 & 6 & 21 \\
\hline 2 & $8-15$ & 70 & 13 & 58 & 8 & $2-14$ & -58 & 11 & 52 \\
\hline 4 & $8-15$ & 0 & 38 & 100 & 10 & $2-14$ & 95 & 39 & 16 \\
\hline 6 & $8-15$ & -58 & 7 & 26 & 12 & $2-14$ & 55 & 61 & $5 b$ \\
\hline & $8-15$ & -49 & 25 & 27 & 14 & $2-14$ & 48 & 35 & 63 \\
\hline 12 & $8-15$ & -60 & 8 & 25 & 1 & $3-14$ & -113 & 29 & 3 \\
\hline 3 & $9-15$ & 49 & 4 & 65 & 3 & $3-14$ & 0 & 37 & 92 \\
\hline 5 & $9-15$ & -78 & 20 & 48 & 5 & $3-14$ & -31 & 47 & 34 \\
\hline 7 & $9-15$ & -78 & 59 & 52 & 7 & $3-14$ & 30 & 53 & 71 \\
\hline 9 & $9-15$ & -71 & 13 & 51 & 9 & $3-14$ & -48 & 10 & 26 \\
\hline 11 & $9-15$ & -73 & 2 & 52 & 11 & $3-14$ & -74 & 46 & 53 \\
\hline 4 & $10-15$ & 49 & 29 & 70 & 13 & $3-14$ & 0 & 1 & 91 \\
\hline 6 & $10-15$ & 49 & 3 & 32 & 15 & $3-14$ & -100 & 35 & 8 \\
\hline 8 & $10-15$ & 87 & 86 & 21 & 2 & $4-14$ & 56 & 28 & $0 y$ \\
\hline 10 & $10-15$ & 50 & 27 & 27 & 4 & $4-14$ & -29 & 27 & 71 \\
\hline 12 & $10-15$ & 61 & 25 & 49 & 6 & $4-14$ & 43 & 48 & 61 \\
\hline 3 & $11-15$ & -134 & 4 & 41 & 8 & $4-14$ & 84 & 9 & 1 \\
\hline 5 & $11-15$ & -73 & 20 & 53 & 10 & $4-14$ & -51 & 19 & \\
\hline 9 & $11-15$ & -113 & 17 & 36 & 12 & $4-14$ & 0 & 61 & 10 \\
\hline 11 & $11-15$ & -66 & 26 & 50 & 14 & $4-14$ & 49 & 12 & 6 \\
\hline 4 & $12-15$ & -66 & 5 & 55 & 1 & $5-14$ & 66 & 22 & 5 \\
\hline 6 & $12-15$ & 55 & 28 & 31 & 3 & $5-14$ & -42 & 1 & 5 \\
\hline 8 & $12-15$ & -80 & 42 & 54 & 5 & $5-14$ & -123 & 6 & 3 \\
\hline 10 & $12-15$ & 59 & 8 & 57 & 7 & $5-14$ & -69 & 27 & 2 \\
\hline 5 & $13-15$ & -82 & 25 & 47 & 9 & $5-14$ & 80 & 10 & 1 \\
\hline 7 & $13-15$ & -66 & 67 & 53 & 11 & $5-14$ & -101 & 29 & 4 \\
\hline 9 & $13-15$ & 95 & 6 & 20 & 13 & $5-14$ & -31 & 61 & 7 \\
\hline 2 & $0-14$ & 32 & 1 & 68 & 2 & $6-14$ & -53 & 27 & 5 \\
\hline 4 & $0-14$ & -59 & 27 & 48 & 4 & $6-14$ & -11 & 26 & \\
\hline 6 & $0-14$ & 73 & 36 & 47 & 6 & $6-14$ & 0 & 18 & \\
\hline 8 & $0-14$ & 52 & 75 & 56 & 8 & $6-14$ & 0 & 4 & \\
\hline & $0-14$ & -41 & 61 & 60 & 10 & $6-14$ & -86 & 8 & \\
\hline
\end{tabular}


Reflexões não observadas $I<3 \sigma(I)$

Values of $10 *$ Fobs and $10{ }^{*}$ Fcalc

Page 3

\begin{tabular}{|c|c|c|c|c|c|c|c|c|c|}
\hline $\mathrm{H}$ & K I & Eobs & Fcalc & SigE & $\mathrm{H}$ & $\mathrm{L}$ & Fobs & Ecalc & $\operatorname{Sig} E$ \\
\hline - & - & ---- & ---- & ---- & - & - & ---- & ---- & ---- \\
\hline 12 & $6-14$ & 0 & 22 & 98 & 11 & $13-14$ & -109 & 19 & 40 \\
\hline 14 & $6-14$ & 69 & 31 & 58 & 4 & $14-14$ & -135 & 44 & 37 \\
\hline 1 & $7-14$ & -140 & 71 & 30 & 6 & $14-14$ & -79 & 69 & 48 \\
\hline 3 & $7-14$ & 87 & 102 & 19 & 8 & $14-14$ & -40 & 43 & 34 \\
\hline 5 & $7-14$ & 54 & 10 & 58 & 10 & $14-14$ & 31 & 6 & 36 \\
\hline 7 & $7-14$ & -68 & 39 & 21 & 5 & $15-14$ & -99 & 46 & 43 \\
\hline 9 & $7-14$ & 23 & 33 & 39 & 7 & $15-14$ & -65 & 11 & 58 \\
\hline 13 & $7-14$ & 90 & 52 & 20 & 9 & $15-14$ & 84 & 3 & 53 \\
\hline 2 & $8-14$ & -100 & 12 & 39 & 6 & $16-14$ & -145 & 44 & 35 \\
\hline 4 & $8-14$ & 78 & 31 & 46 & 8 & $16-14$ & -173 & 28 & 30 \\
\hline 6 & $8-14$ & 24 & 41 & 42 & 2 & $0-13$ & -76 & 0 & 41 \\
\hline 8 & $8-14$ & -72 & 6 & 21 & 4 & $0-13$ & -84 & 0 & 42 \\
\hline 10 & $8-14$ & -43 & 6 & 54 & 6 & $0-13$ & 77 & 0 & 21 \\
\hline 12 & $8-14$ & -44 & 65 & 60 & 8 & $0-13$ & -62 & 0 & 53 \\
\hline 14 & $8-14$ & 50 & 3 & 63 & 10 & $0-13$ & 59 & 0 & 46 \\
\hline 1 & $9-14$ & 18 & 32 & 48 & 12 & $0-13$ & -42 & 0 & 63 \\
\hline 3 & $9-14$ & 47 & 54 & 70 & 14 & $0-13$ & -44 & 0 & 52 \\
\hline 5 & $9-14$ & 64 & 19 & 24 & 1 & $1-13$ & -33 & 9 & 32 \\
\hline 7 & $9-14$ & 52 & 67 & 30 & 3 & $1-13$ & -110 & 38 & 29 \\
\hline 9 & $9-14$ & -81 & 43 & 41 & 7 & $1-13$ & -73 & 12 & 24 \\
\hline 11 & $9-14$ & 71 & 72 & 22 & 9 & $1-13$ & -63 & 14 & 51 \\
\hline 13 & $9-14$ & 60 & 7 & 50 & 11 & $1-13$ & 52 & 32. & 54 \\
\hline 2 & $10-14$ & -45 & 2 & 60 & 13 & $1-13$ & 32 & 9 & 74 \\
\hline 4 & $10-14$ & -129 & 1 & 30 & 15 & $1-13$ & -72 & 19 & 49 \\
\hline 6 & $10-14$ & 32 & 5 & 72 & 2 & $2-13$ & -58 & 61 & 52 \\
\hline 8 & $10-14$ & -43 & 39 & 61 & 4 & $2-13$ & -65 & 9 & 26 \\
\hline 10 & $10-14$ & 21 & 38 & 41 & 8 & $2-13$ & -119 & 71 & 34 \\
\hline 12 & $10-14$ & -64 & 38 & 55 & 10 & $2-13$ & -53 & 31 & 24 \\
\hline 1 & $11-14$ & 35 & 16 & 71 & 12 & $2-13$ & -10 & 10 & 46 \\
\hline 3 & $11-14$ & -46 & 10 & 28 & 14 & $2-13$ & -31 & 24 & 73 \\
\hline 5 & $11-14$ & -99 & 11 & 41 & 1 & $3-13$ & 79 & 57 & 19 \\
\hline 7 & $11-14$ & 73 & 7 & 52 & 5 & $3-13$ & 28 & 121 & 80 \\
\hline 9 & $11-14$ & 17 & 15 & 44 & 7 & $3-13$ & 12 & 39 & $5 !$ \\
\hline 11 & $11-14$ & -45 & 69 & 64 & 9 & $3-13$ & 65 & 46 & 54 \\
\hline 13 & $11-14$ & -74 & 21 & 24 & 13 & $3-13$ & -53 & 13 & 54 \\
\hline 2 & $12-14$ & -56 & 14 & 57 & 15 & $3-13$ & -86 & 28 & 42 \\
\hline 4 & $12-14$ & 48 & 31 & 65 & 4 & $4-13$ & 82 & 26 & 48 \\
\hline 8 & $12-14$ & 79 & 84 & 23 & 6 & $4-13$ & 59 & 82 & $2 \hat{9}$ \\
\hline 10 & $12-14$ & -55 & 46 & 56 & 8 & $4-13$ & -25 & 47 & 42 \\
\hline 12 & $12-14$ & -136 & 36 & 36 & 10 & $4-13$ & -10 & 3 & 45 \\
\hline 3 & $13-14$ & -135 & 1 & 37 & 12 & $4-13$ & 0 & 141 & 110 \\
\hline 5 & $13-14$ & 34 & 43 & 71 & 14 & $4-13$ & 92 & 48 & 18 \\
\hline 7 & $13-14$ & 58 & 30 & 64 & 1 & $5-13$ & -46 & 63 & 26 \\
\hline 9 & $13-14$ & -120 & 30 & 42 & 3 & $5-13$ & -57 & 21 & 56 \\
\hline
\end{tabular}


Reflexões não observadas $I<3 \sigma(I)$

Values of $10 *$ Fobs and $10 *$ Fcalc

Page 4

\begin{tabular}{|c|c|c|c|c|c|c|c|c|}
\hline K & Fobs & Ecalc & SigF & $\mathrm{H}$ & K & Fobs & Fcalc & SigF \\
\hline - & ---- & ----- & ---- & - & - & ---- & ---- & ---- \\
\hline $5-13$ & -113 & 47 & 41 & 11 & $11-13$ & -98 & 29 & 41 \\
\hline $5-13$ & -56 & 30 & 58 & 13 & $11-13$ & -45 & 22 & 69 \\
\hline $5-13$ & -64 & 13 & 42 & 2 & $12-13$ & 66 & 12 & D \\
\hline $5-13$ & 49 & 73 & 27 & 4 & $12-13$ & 61 & 46 & 27 \\
\hline $5-13$ & 57 & 39 & 58 & 6 & $12-13$ & -90 & 50 & 41 \\
\hline $5-13$ & -32 & 17 & 74 & 8 & $12-13$ & 0 & 70 & 105 \\
\hline $6-13$ & 69 & 81 & 49 & 10 & $12-13$ & 73 & 5 & 45 \\
\hline $6-13$ & 0 & 18 & 92 & 12 & $12-13$ & -120 & 90 & 3 \\
\hline $6-13$ & -62 & 17 & 26 & 1 & $13-13$ & -32 & 10 & 1 \\
\hline $6-13$ & 65 & 9 & 44 & 3 & $13-13$ & 62 & 15 & 5 \\
\hline $6-13$ & -65 & 0 & 45 & 5 & $13-13$ & -53 & 42 & \\
\hline $6-13$ & 59 & 3 & 25 & 7 & $13-13$ & -43 & 64 & \\
\hline $7-13$ & 80 & 94 & 21 & 9 & $13-13$ & -32 & 42 & 35 \\
\hline $7-13$ & 70 & 57 & 21 & 11 & $13-13$ & -71 & 43 & \\
\hline $7-13$ & -79 & 15 & 19 & 13 & $13-13$ & -105 & 55 & בע \\
\hline $7-13$ & 43 & 10 & 29 & 2 & $14-13$ & 34 & 28 & $J$ \\
\hline $7-13$ & -40 & 13 & 54 & 4 & $14-13$ & -109 & 17 & 35 \\
\hline $7-13$ & -62 & 2 & 53 & 6 & $14-13$ & 80 & 10 & 19 \\
\hline $7-13$ & -57 & 50 & 53 & 8 & $14-13$ & -69 & 10 & 51 \\
\hline $8-13$ & -52 & 13 & 55 & 10 & $14-13$ & 0 & 24 & 8 \\
\hline $8-13$ & -71 & 11 & 48 & 12 & $14-13$ & 94 & 82 & $\angle 1$ \\
\hline $8-13$ & -57 & 64 & 53 & 1 & $15-13$ & -105 & 43 & 15 \\
\hline $8-13$ & -87 & 54 & 40 & 3 & $15-13$ & 68 & 28 & 5 \\
\hline $8-13$ & 31 & 106 & 77 & 5 & $15-13$ & 44 & 20 & 31 \\
\hline $8-13$ & 73 & 3 & 47 & 7 & $15-13$ & -63 & 26 & 57 \\
\hline $8-13$ & -65 & 18 & 51 & 9 & $15-13$ & -111 & 46 & 37 \\
\hline $9-13$ & -50 & 48 & 27 & 11 & $15-13$ & -65 & 18 & 2. \\
\hline $9-13$ & -66 & 3 & 49 & 4 & $16-13$ & 0 & 6 & 97 \\
\hline $9-13$ & 75 & 9 & 48 & 6 & $16-13$ & -170 & 0 & 30 \\
\hline $9-13$ & 43 & 81 & 63 & 8 & $16-13$ & 0 & 3 & 92 \\
\hline $9-13$ & -83 & 61 & 40 & 10 & $16-13$ & -17 & 31 & 44 \\
\hline $9-13$ & 83 & 89 & 21 & 5 & $17-13$ & -80 & 6 & 49 \\
\hline $9-13$ & 77 & 8 & 21 & 7 & $17-13$ & -122 & 12 & $J$ \\
\hline $210-13$ & -92 & 2 & 36 & 9 & $17-13$ & -46 & 62 & 60 \\
\hline $410-13$ & -43 & 14 & 29 & 4 & $18-13$ & -88 & 70 & 4 \\
\hline $610-13$ & 90 & 70 & 18 & 6 & $18-13$ & 60 & 12 & 5 \\
\hline $810-13$ & -10 & 48 & 47 & 8 & $18-13$ & -12 & 18 & 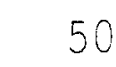 \\
\hline $010-13$ & -30 & 77 & 71 & 2 & $0-12$ & -47 & 27 & - \\
\hline $210-13$ & -94 & 31 & 42 & 8 & $0-12$ & -80 & 4 & 4 \\
\hline $410-13$ & 0 & 5 & 93 & 10 & $0-12$ & -61 & 30 & 5 \\
\hline $111-13$ & -118 & 13 & 33 & 12 & $0-12$ & -71 & 53 & 4 \\
\hline $311-13$ & -28 & 18 & 35 & 14 & $0-12$ & -43 & 20 & 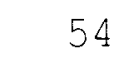 \\
\hline $511-13$ & 63 & 32 & 53 & 3 & $1-12$ & -46 & 6 & 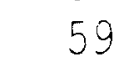 \\
\hline $911-13$ & -100 & 20 & 38 & 9 & $1-12$ & 74 & 58 & 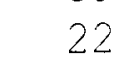 \\
\hline
\end{tabular}


Reflexões não observadas $I<3 \sigma$ (I)

Values of $10 *$ Fobs and $10 *$ Ecalc

Page 5

\begin{tabular}{|c|c|c|c|c|c|c|c|c|c|}
\hline $\mathrm{H}$ & K & Eobs & Ecalc & SigF & $\mathrm{H}$ & K & Fobs & Fcalc & $\operatorname{Sig} F$ \\
\hline - & - & ---- & ---- & ---- & - & - & ---- & ---- & ---- \\
\hline 13 & $1-12$ & -94 & 14 & 38 & 1 & $9-12$ & 56 & 15 & 25 \\
\hline 15 & $1-12$ & 84 & 90 & 21 & 3 & $9-12$ & -75 & 45 & 23 \\
\hline 2 & $2-12$ & 93 & 76 & 19 & 5 & $9-12$ & 50 & 40 & 64 \\
\hline 4 & $2-12$ & -70 & 44 & 49 & 7 & $9-12$ & -39 & 9 & 62 \\
\hline 8 & $2-12$ & -75 & 43 & 46 & 9 & $9-12$ & -26 & 26 & 33 \\
\hline 10 & $2-12$ & -87 & 32 & 43 & 11 & $9-12$ & 54 & 54 & 51 \\
\hline 12 & $2-12$ & -82 & 63 & 40 & 13 & $9-12$ & -88 & 51 & $\leq 1$ \\
\hline 14 & $2-12$ & -69 & 44 & 44 & 15 & $9-12$ & -27 & 16 & 37 \\
\hline 1 & $3-12$ & 82 & 82 & 49 & 2 & $10-12$ & -65 & 10 & 45 \\
\hline 3 & $3-12$ & -49 & 45 & 30 & 4 & $10-12$ & 72 & 98 & 55 \\
\hline 7 & $3-12$ & -84 & 32 & 46 & 6 & $10-12$ & 59 & 103 & 65 \\
\hline 9 & $3-12$ & 67 & 17 & 23 & 8 & $10-12$ & 58 & 54 & 44 \\
\hline 11 & $3-12$ & 69 & 64 & 25 & 10 & $10-12$ & -33 & 44 & 31 \\
\hline 13 & $3-12$ & 73 & 66 & 22 & 12 & $10-12$ & -97 & 59 & 42 \\
\hline 2 & $4-12$ & -73 & 65 & 51 & 14 & $10-12$ & -138 & 7 & 33 \\
\hline 4 & $4-12$ & -46 & 63 & 60 & 1 & $11-12$ & -73 & 50 & 49 \\
\hline 8 & $4-12$ & -81 & 34 & 40 & 3 & $11-12$ & -75 & 10 & 18 \\
\hline 10 & $4-12$ & -33 & 9 & 35 & 5 & $11-12$ & 0 & 28 & 93 \\
\hline 12 & $4-12$ & -71 & 38 & 45 & 7 & $11-12$ & -70 & 11 & 44 \\
\hline 14 & $4-12$ & 69 & 24 & 23 & 9 & $11-12$ & -42 & 5 & 28 \\
\hline 3 & $5-12$ & 93 & 56 & 20 & 11 & $11-12$ & 64 & 31 & 48 \\
\hline 5 & $5-12$ & 100 & 101 & 17 & 13 & $11-12$ & -84 & 57 & 45 \\
\hline 7 & $5-12$ & 27 & 28 & 72 & 2 & $12-12$ & -73 & 44 & 48 \\
\hline 9 & $5-12$ & 55 & 41 & 26 & 4 & $12-12$ & 53 & 75 & 52 \\
\hline 11 & $5-12$ & 83 & 93 & 19 & 6 & $12-12$ & -50 & 50 & 55 \\
\hline 13 & $5-12$ & -74 & 13 & 43 & 8 & $12-12$ & 43 & 7 & 28 \\
\hline 4 & $6-12$ & -64 & 20 & 24 & 10 & $12-12$ & -42 & 94 & 66 \\
\hline 6 & $6-12$ & -130 & 84 & 33 & 12 & $12-12$ & 66 & 29 & 58 \\
\hline 8 & $6-12$ & 56 & 68 & 54 & 14 & $12-12$ & 30 & 26 & 38 \\
\hline 10 & $6-12$ & -48 & 85 & 63 & 1 & $13-12$ & -30 & 134 & 78 \\
\hline 12 & $6-12$ & 88 & 96 & 19 & 3 & $13-12$ & -19 & 56 & 42 \\
\hline 14 & $6-12$ & 18 & 66 & 47 & 5 & $13-12$ & 86 & 75 & 20 \\
\hline 3 & $7-12$ & -48 & 15 & 60 & 7 & $13-12$ & 62 & 24 & 55 \\
\hline 5 & $7-12$ & -72 & 8 & 45 & 9 & $13-12$ & -85 & 39 & 43 \\
\hline 7 & $7-12$ & -22 & 47 & 42 & 13 & $13-12$ & 61 & 96 & 28 \\
\hline 9 & $7-12$ & -68 & 61 & 43 & 4 & $14-12$ & -45 & 21 & 28 \\
\hline 13 & $7-12$ & 73 & 7 & 53 & 6 & $14-12$ & 31 & 43 & 79 \\
\hline 15 & $7-12$ & 69 & 104 & 62 & 8 & $14-12$ & -100 & 38 & 39 \\
\hline 2 & $8-12$ & 67 & 74 & 50 & 10 & $14-12$ & 0 & 82 & 94 \\
\hline 4 & $8-12$ & -104 & 32 & 41 & 3 & $15-12$ & 65 & 72 & 5 \\
\hline 6 & $8-12$ & 63 & 79 & 60 & 5 & $15-12$ & -30 & 46 & 67 \\
\hline 8 & $8-12$ & 85 & 61 & 19 & 7 & $15-12$ & 55 & 0 & 50 \\
\hline 12 & $8-12$ & -49 & 7 & 26 & 9 & $15-12$ & -93 & 31 & \\
\hline 14 & $8-12$ & -31 & 18 & 65 & 13 & $15-12$ & -74 & 20 & 3 \\
\hline
\end{tabular}


Reflexões não observadas $I<3 \sigma$ (I)

Values of $10^{*}$ Eobs and $10^{*}$ Ecalc

Page 6

\begin{tabular}{|c|c|c|c|c|c|c|c|c|}
\hline K I & Fobs & Ecalc & $\operatorname{SigF}$ & $\mathrm{H}$ & $\mathrm{L}$ & Fobs & Ecalc & $\operatorname{Sig} E$ \\
\hline- & ---- & ----- & ---- & - & - & ---- & ---- & ---- \\
\hline $416-12$ & 59 & 83 & 26 & 7 & $5-11$ & 67 & 12 & 17 \\
\hline $6 \quad 16-12$ & 27 & 3 & 38 & 9 & $5-11$ & 49 & 3 & 27 \\
\hline $816-12$ & -82 & 59 & 48 & 11 & $5-11$ & -27 & 64 & 71 \\
\hline $1016-12$ & 73 & 101 & 51 & 2 & $6-11$ & 27 & 44 & 75 \\
\hline $317-12$ & -44 & 1 & 28 & 4 & $6-11$ & 70 & 113 & 47 \\
\hline $5 \quad 17-12$ & 81 & 1 & 48 & 6 & $6-11$ & 54 & 27 & 21 \\
\hline $7 \quad 17-12$ & -59 & 15 & 24 & 8 & $6-11$ & -50 & 7 & 25 \\
\hline $9 \quad 17-12$ & -36 & 15 & 32 & 10 & $6-11$ & -102 & 36 & 40 \\
\hline $4 \quad 18-12$ & 13 & 3 & 50 & 12 & $6-11$ & -112 & 10 & 35 \\
\hline $618-12$ & 77 & 19 & 19 & 16 & $6-11$ & -57 & 7 & 60 \\
\hline $818-12$ & -78 & 51 & 42 & 3 & $7-11$ & -32 & 39 & 35 \\
\hline $5 \quad 19-12$ & -32 & 51 & 68 & 5 & $7-11$ & 90 & 43 & 15 \\
\hline $719-12$ & -108 & 20 & 38 & 7 & $7-11$ & 78 & 58 & 18 \\
\hline $420-12$ & -12 & 59 & 49 & 11 & $7-11$ & 84 & 23 & 49 \\
\hline $620-12$ & 89 & 75 & 20 & 13 & $7-11$ & 76 & 37 & 20 \\
\hline $820-12$ & -105 & 49 & 44 & 15 & $7-11$ & -129 & 24 & 34 \\
\hline $20-11$ & -81 & 0 & 41 & 4 & $8-11$ & 77 & 108 & 54 \\
\hline $0-11$ & -50 & 0 & 53 & 8 & $8-11$ & 0 & 72 & 92 \\
\hline $0-11$ & -109 & 0 & 32 & 10 & $8-11$ & -55 & 24 & 28 \\
\hline $0-11$ & 55 & 0 & 21 & 12 & $8-11$ & 69 & 36 & 43 \\
\hline $0-11$ & -110 & 0 & 36 & 14 & $8-11$ & 90 & 55 & 17 \\
\hline $0-11$ & 51 & 0 & 56 & 1 & $9-11$ & 58 & 33 & 57 \\
\hline $0-11$ & -121 & 0 & 30 & 3 & $9-11$ & -104 & 18 & 36 \\
\hline $0-11$ & 49 & 0 & 57 & 7 & $9-11$ & 47 & 38 & 62 \\
\hline $1-11$ & 66 & 12 & 48 & 9 & $9-11$ & -99 & 5 & 39 \\
\hline $1-11$ & -62 & 5 & 43 & 11 & $9-11$ & 12 & 46 & 48 \\
\hline $1-11$ & -82 & 36 & 43 & 13 & $9-11$ & 37 & 3 & 32 \\
\hline $1-11$ & -65 & 5 & 50 & 2 & $10-11$ & 89 & 79 & 20 \\
\hline $2-11$ & -50 & 28 & 22 & 4 & $10-11$ & 69 & 35 & 52 \\
\hline $2-11$ & 87 & 68 & 15 & 8 & $10-11$ & 100 & 105 & 18 \\
\hline $2-11$ & -98 & 29 & 40 & 10 & $10-11$ & -80 & 68 & 46 \\
\hline $2-11$ & 62 & 33 & 24 & 12 & $10-11$ & 66 & 57 & 22 \\
\hline $2-1 i$ & 92 & 103 & 20 & 14 & $10-11$ & -55 & 24 & 51 \\
\hline $3-11$ & -57 & 30 & 50 & 1 & $11-11$ & 86 & 5 & 15 \\
\hline $3-11$ & 0 & 2 & 90 & 3 & $11-11$ & 59 & 69 & 28 \\
\hline $3-11$ & -135 & 15 & 29 & 7 & $11-11$ & 70 & 39 & 55 \\
\hline $4-11$ & -25 & 84 & 69 & 9 & $11-11$ & -94 & 67 & 41 \\
\hline $4-I I$ & 52 & 15 & 52 & 11 & $11-1 \hat{1}$ & 0 & 11 & 95 \\
\hline $4-11$ & -35 & 54 & 59 & 13 & $11-11$ & -76 & 5 & 44 \\
\hline $4-11$ & 53 & 42 & 49 & 15 & $11-11$ & -88 & 121 & 52 \\
\hline $4-11$ & 30 & 42 & 72 & 2 & $12-11$ & -157 & 17 & 25 \\
\hline $4-11$ & 60 & 64 & 27 & 4 & $12-11$ & 48 & 49 & 33 \\
\hline $5-11$ & 13 & 14 & 42 & 6 & $12-11$ & 73 & 95 & 26 \\
\hline $5 \quad 5-11$ & 52 & 92 & 54 & 8 & $12-1$ & 57 & 19 & 51 \\
\hline
\end{tabular}


Reflexões não observadas $I<3 \sigma(I)$

Values of $10 *$ Fobs and $10^{*} \mathrm{ECalC}$

Page 7

\begin{tabular}{|c|c|c|c|c|c|c|c|c|c|}
\hline $\mathrm{H}$ & K I & Fobs & Fcalc & SigE & $\mathrm{H}$ & L & Fobs & Fcalc & $\operatorname{Sig} F$ \\
\hline - & - & ---- & --- & ---- & - & - & ---- & ----- & --- \\
\hline 10 & $12-11$ & -71 & 27 & 49 & 6 & $22-11$ & -41 & 13 & 31 \\
\hline 14 & $12-11$ & -92 & 29 & 41 & 2 & $0-10$ & -54 & 91 & 49 \\
\hline 1 & $13-11$ & -59 & 1 & 49 & 8 & $0-10$ & 64 & 90 & 17 \\
\hline 3 & $13-11$ & 79 & 81 & 20 & 10 & $0-10$ & 46 & 8 & 50 \\
\hline 7 & $13-11$ & -80 & 33 & 44 & 12 & $0-10$ & -99 & 12 & 39 \\
\hline 9 & $13-11$ & -28 & 38 & 68 & 7 & $1-10$ & -67 & 26 & 38 \\
\hline 11 & $13-11$ & 90 & 103 & 19 & 9 & $1-10$ & 54 & 12 & 21 \\
\hline 13 & $13-11$ & 74 & 73 & 22 & 11 & $1-10$ & -70 & 62 & 46 \\
\hline 2 & $14-11$ & -29 & 77 & 69 & 13 & $1-10$ & 43 & 42 & 56 \\
\hline 4 & $14-11$ & 95 & 93 & 17 & 4 & $2-10$ & -23 & 44 & 64 \\
\hline 6 & $14-11$ & -81 & 1 & 41 & 6 & $2-10$ & 48 & 12 & 49 \\
\hline 8 & $14-11$ & -71 & 12 & 46 & 14 & $2-10$ & -42 & 46 & 60 \\
\hline 10 & $14-11$ & 54 & 78 & 54 & 16 & $2-10$ & -60 & 31 & 23 \\
\hline 12 & $14-11$ & 77 & 65 & 23 & 1 & $3-10$ & 39 & 56 & 26 \\
\hline 14 & $14-11$ & -115 & 34 & 40 & 3 & $3-10$ & 49 & 92 & 51 \\
\hline 1 & $15-11$ & -133 & 72 & 32 & 5 & $3-10$ & 64 & 112 & 42 \\
\hline 3 & $15-11$ & 93 & 113 & 18 & 7 & $3-10$ & 30 & 30 & 28 \\
\hline 5 & $15-11$ & -105 & 63 & 38 & 9 & $3-10$ & 68 & 81 & 18 \\
\hline 7 & $15-11$ & -29 & 42 & 34 & 11 & $3-10$ & 97 & 22 & 17 \\
\hline 9 & $15-11$ & 69 & 46 & 42 & 13 & $3-10$ & 62 & 99 & 24 \\
\hline 13 & $15-11$ & -32 & 45 & 69 & 2 & $4-10$ & 76 & 107 & 46 \\
\hline 2 & $16-11$ & -58 & 23 & 24 & 4 & $4-10$ & 78 & 94 & 15 \\
\hline 4 & $16-11$ & -89 & 15 & 16 & 6 & $4-10$ & 56 & 2 & 19 \\
\hline 6 & $16-11$ & -99 & 2 & 40 & 8 & $4-10$ & 57 & 43 & 46 \\
\hline 8 & $16-11$ & 69 & 17 & 44 & 12 & $4-10$ & -39 & 3 & 63 \\
\hline 1 & $17-11$ & 47 & 42 & 30 & 1 & $5-10$ & 59 & 14 & 20 \\
\hline 5 & $17-11$ & -67 & 21 & 47 & 5 & $5-10$ & 0 & 53 & 83 \\
\hline 7 & $17-11$ & -52 & 69 & 55 & 7 & $5-10$ & -42 & 52 & 53 \\
\hline 12 & $17-11$ & 74 & 14 & 44 & 2 & $6-10$ & 52 & 13 & 52 \\
\hline 4 & $18-11$ & -30 & 6 & 63 & 4 & $6-10$ & -39 & 13 & 26 \\
\hline 6 & $18-11$ & -43 & 27 & 62 & 6 & $6-10$ & -43 & 1 & 23 \\
\hline 8 & $18-11$ & 27 & 51 & 38 & 10 & $6-10$ & -65 & 32 & 23 \\
\hline 2 & $19-11$ & -45 & 33 & 67 & 12 & $6-10$ & -69 & 11 & 50 \\
\hline 3 & $19-11$ & 33 & 21 & 63 & 16 & $6-10$ & -46 & 88 & 60 \\
\hline 5 & $19-11$ & -31 & 3 & 64 & 3 & $7-10$ & 69 & 2 & 41 \\
\hline 7 & $19-11$ & -77 & 16 & 40 & 5 & $7-10$ & 25 & 58 & 70 \\
\hline 11 & $19-11$ & -46 & 6 & 62 & 7 & $7-10$ & -93 & 9 & 33 \\
\hline$\leq$ & $20-11$ & -55 & 8 & 51 & 13 & $7-10$ & -102 & 15 & 34 \\
\hline 6 & $20-11$ & -64 & 72 & 56 & 2 & $8-10$ & 27 & 36 & 68 \\
\hline 10 & $20-11$ & 58 & 74 & 25 & 4 & $8-10$ & -24 & 41 & 67 \\
\hline 3 & $21-11$ & 69 & 27 & 24 & 6 & $8-10$ & -35 & 31 & 62 \\
\hline 5 & $21-11$ & -92 & 37 & 43 & 8 & $8-10$ & -25 & 54 & 69 \\
\hline 7 & $21-11$ & -44 & 0 & 29 & 12 & $8-10$ & 58 & 74 & 29 \\
\hline$S$ & $22-11$ & -47 & 32 & 52 & 14 & $8-10$ & -43 & 49 & 50 \\
\hline
\end{tabular}


Reflexões não observadas $I<3 \sigma$ (I)

Values of $10 *$ Fobs and $10 *$ Fcalc

Page 8

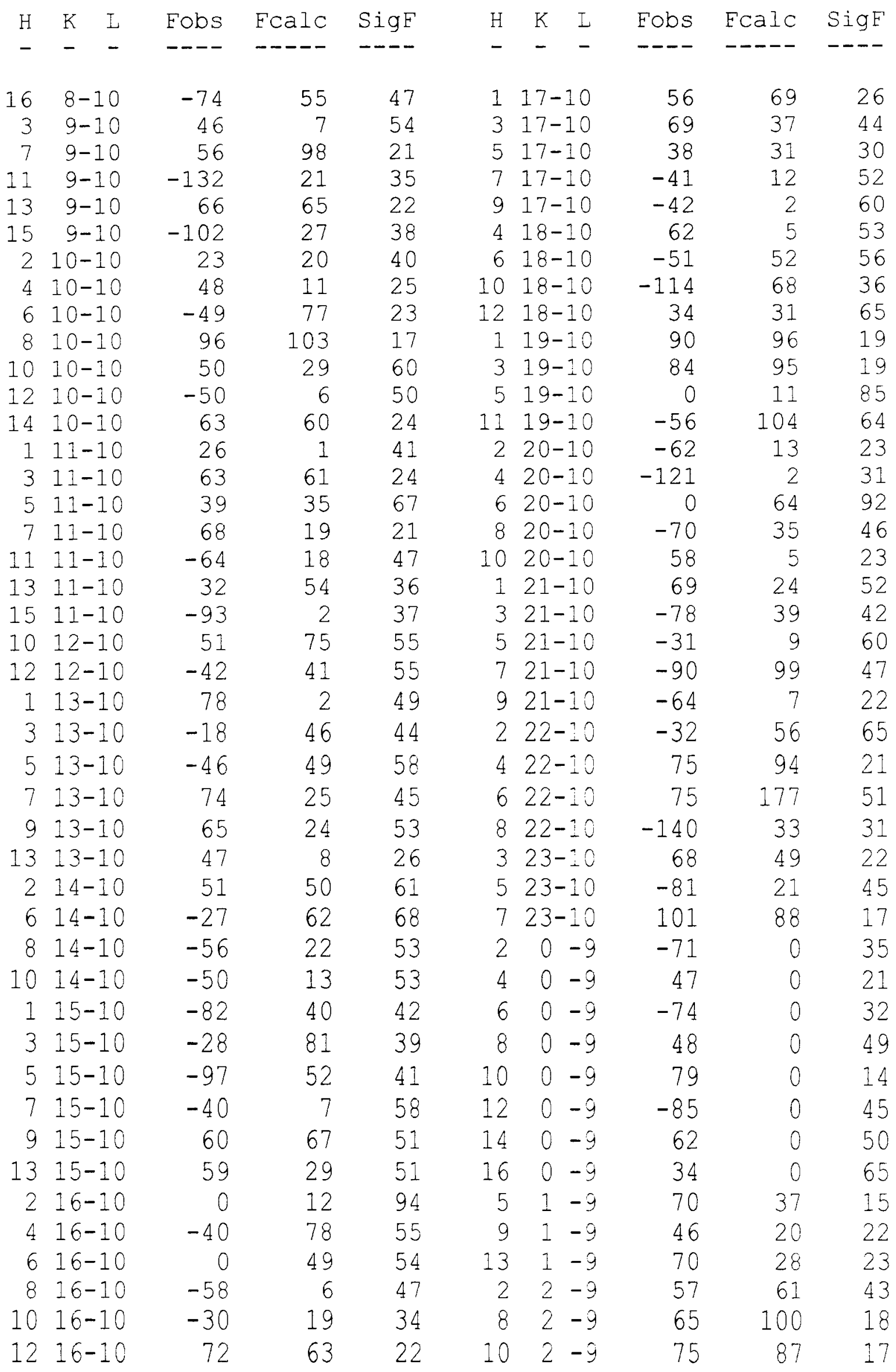


Reflexões não observadas $I<3 \sigma$ (I)

Values of $10 *$ Fobs and $10 * \mathrm{Fcalc}$

Page 9

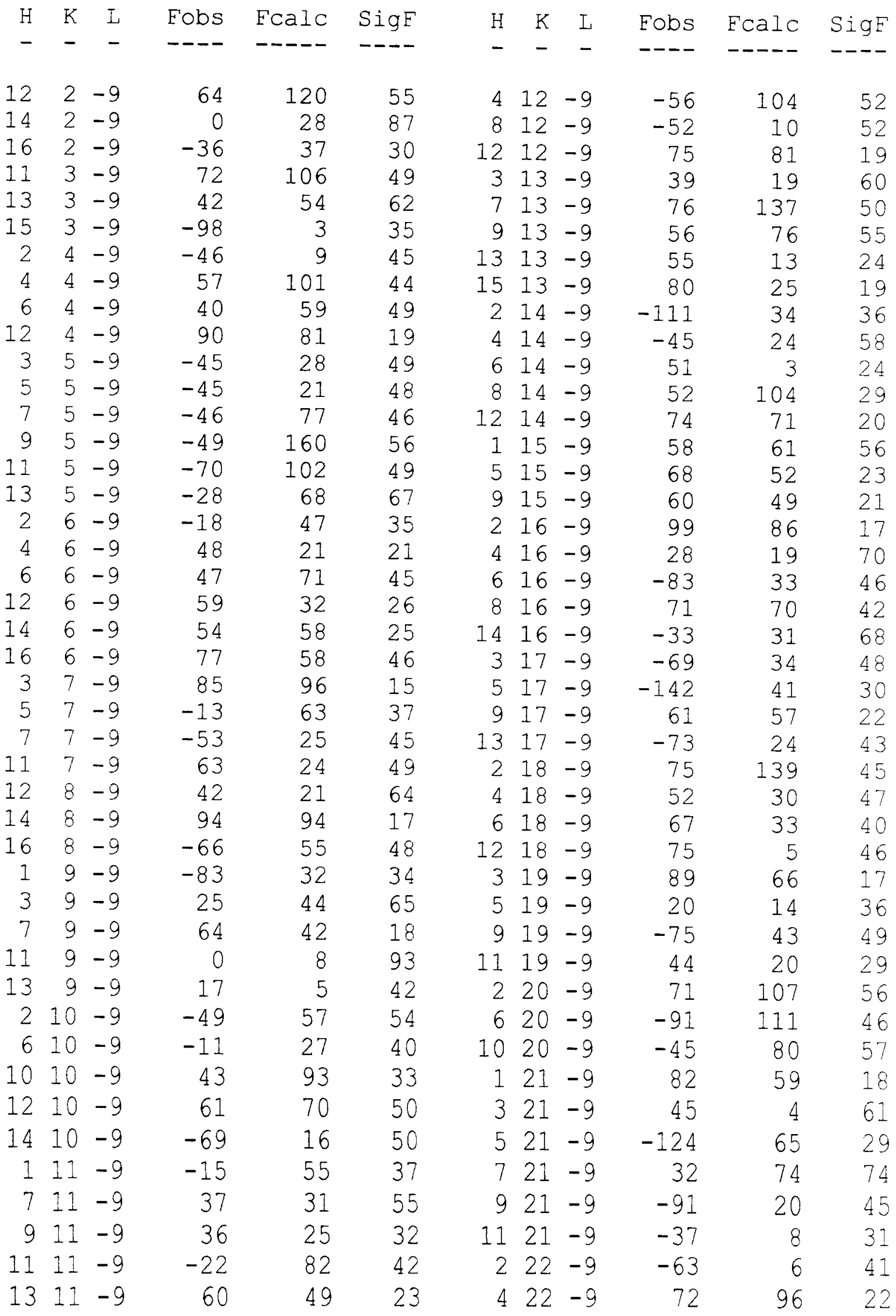


Reflexões não observadas I < $3 \sigma$ (I)

Values of $10^{*}$ Fobs and $10^{*}$ Ecalc

Page 10

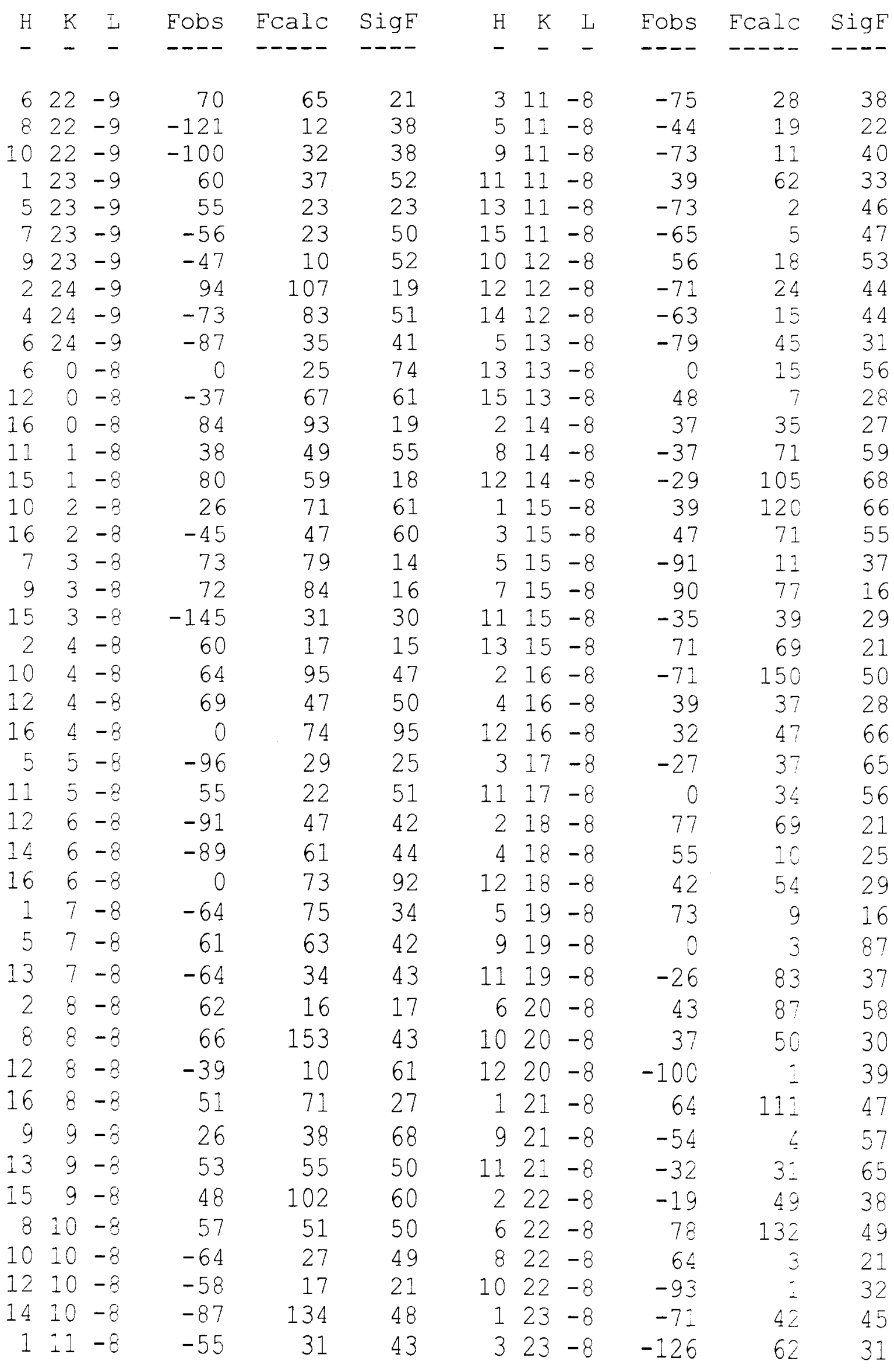


Reflexões não observadas $I<3 \sigma$ (I)

Values of $10 *$ Fobs and $10 *$ Ecalc

Page 11

\begin{tabular}{|c|c|c|c|c|c|c|c|c|c|c|c|}
\hline $\mathrm{H}$ & K & L & Fobs & Fcalc & SigE & $\mathrm{H}$ & K & L & Fobs & Fcalc & SigF \\
\hline - & - & - & ---- & ----- & ---- & - & - & - & ---- & ---- & ---- \\
\hline 52 & $23-$ & -8 & 60 & 26 & 22 & 11 & 9 & -7 & 39 & 74 & 63 \\
\hline 92 & $23-$ & -8 & 69 & 49 & 21 & 13 & 9 & -7 & 31 & 50 & 58 \\
\hline 22 & 24 & -8 & -45 & 52 & 55 & 15 & 9 & -7 & 60 & 31 & 23 \\
\hline 42 & 24 & -8 & 36 & 61 & 30 & 10 & 10 & -7 & -101 & 62 & 35 \\
\hline 62 & 24 & -8 & 33 & 13 & 66 & 12 & 10 & -7 & 42 & 2 & 61 \\
\hline 8 & 24 & -8 & 48 & 16 & 49 & 14 & 10 & -7 & 32 & 42 & 59 \\
\hline 12 & 25 & -8 & 49 & 57 & 60 & 5 & 11 & -7 & 20 & 41 & 31 \\
\hline 32 & 25 & -8 & 55 & 5 & 24 & 9 & 11 & -7 & 58 & 74 & 19 \\
\hline 5 & 25 & -8 & -46 & 21 & 54 & 11 & 11 & -7 & -47 & 11 & 57 \\
\hline 72 & 25 & -3 & 69 & 10 & 42 & 15 & 11 & -7 & 72 & 93 & 22 \\
\hline 2 & 0. & -7 & 53 & 0 & 39 & 6 & 12 & -7 & 56 & 63 & 18 \\
\hline 4 & 0 & -7 & 20 & 0 & 27 & 8 & 12 & -7 & 80 & 82 & 15 \\
\hline 6 & 0 & -7 & -60 & 0 & 34 & 14 & 12 & -7 & -44 & 0 & 56 \\
\hline 8 & 0. & -7 & 56 & 0 & 37 & 5 & 13 & -7 & 49 & 25 & 19 \\
\hline 10 & 0 & -7 & 42 & 0 & 21 & 11 & 13 & -7 & -124 & 94 & 32 \\
\hline 12 & 0 & -7 & -99 & 0 & 35 & 15 & 13 & -7 & -88 & 1 & 41 \\
\hline 14 & 0 & -7 & -109 & 0 & 32 & 4 & 14 & -7 & -28 & 10 & 27 \\
\hline 16 & 0 & -7 & -102 & 0 & 35 & 6 & 14 & -7 & 36 & 23 & 52 \\
\hline 1 & 1 & -7 & 57 & 24 & 14 & 12 & 14 & -7 & -104 & 3 & 34 \\
\hline 9 & 1. & -7 & 34 & 14 & 25 & 14 & 14 & -7 & -107 & 16 & 36 \\
\hline 11 & 1. & -7 & -48 & 20 & 21 & 7 & 15 & -7 & 70 & 101 & 43 \\
\hline 15 & 1 & -7 & -53 & 145 & 56 & 13 & 15 & -7 & 0 & 148 & 101 \\
\hline 12 & 2 & -7 & -65 & 21 & 45 & 4 & 16 & -7 & -15 & 45 & 35 \\
\hline 6 & 2 & -7 & 0 & 69 & 95 & 6 & 16 & -7 & 54 & 65 & 47 \\
\hline 7 & 3 & -7 & 63 & 106 & 41 & 12 & 16 & -7 & 64 & 51 & 47 \\
\hline$i$ & 3 & -7 & 27 & 79 & 67 & 14 & 16 & -7 & -33 & 53 & 63 \\
\hline 2 & 4 & -7 & 21 & 61 & 55 & 3 & 17 & -7 & 83 & 94 & 17 \\
\hline 0 & 4 & -7 & -24 & 46 & 64 & 9 & 17 & -7 & -46 & 39 & 25 \\
\hline 14 & 4 & -7 & 72 & 64 & 20 & 11 & 17 & -7 & 55 & 54 & 50 \\
\hline 16 & 4 & -7 & 97 & 79 & 17 & 13 & 17 & -7 & 65 & 29 & 21 \\
\hline 5 & 5 & -7 & -49 & 36 & 36 & 2 & 18 & -7 & 40 & 15 & 59 \\
\hline 7 & 5. & -7 & -37 & 72 & 45 & 4 & 18 & -7 & 30 & 25 & 35 \\
\hline 15 & 5. & -7 & 57 & 93 & 55 & 6 & 18 & -7 & 23 & 40 & 41 \\
\hline 2 & 6 & -7 & 61 & 39 & 36 & 12 & 18 & -7 & 0 & 74 & 94 \\
\hline 10 & 6 & -7 & 46 & 76 & 23 & 3 & 19 & -7 & 93 & 63 & 16 \\
\hline 16 & 6 & -7 & 57 & 3 & 22 & 5 & 19 & -7 & -93 & 8 & 37 \\
\hline 16 & 6 & -7 & -46 & 6 & 51 & 9 & 19 & -7 & 0 & 67 & 57 \\
\hline 1 & 7 & -7 & 61 & 106 & 16 & 11 & 19 & -7 & -74 & 2 & 18 \\
\hline 3 & 7 & -7 & 58 & 8 & 40 & 2 & 20 & -7 & 30 & 39 & 60 \\
\hline 11 & 7 & -7 & -74 & 8 & 40 & 4 & 20 & -7 & 42 & 58 & 54 \\
\hline 13 & 7 & -7 & -91 & 62 & 34 & 8 & 20 & -7 & -95 & 2 & 31 \\
\hline 8 & 8 & -7 & -117 & 13 & 27 & 10 & 20 & -7 & -104 & 36 & 34 \\
\hline 14 & 8 & -7 & 86 & 64 & 17 & 12 & 20 & -7 & -32 & 7 & 58 \\
\hline 16 & 8 & -7 & 67 & 88 & 24 & 1 & 21 & -7 & 0 & 78 & 85 \\
\hline
\end{tabular}


Reflexões não observadas I < $3 \sigma(I)$

Values of $10 *$ Fobs and $10 *$ Fcalc

Page 12

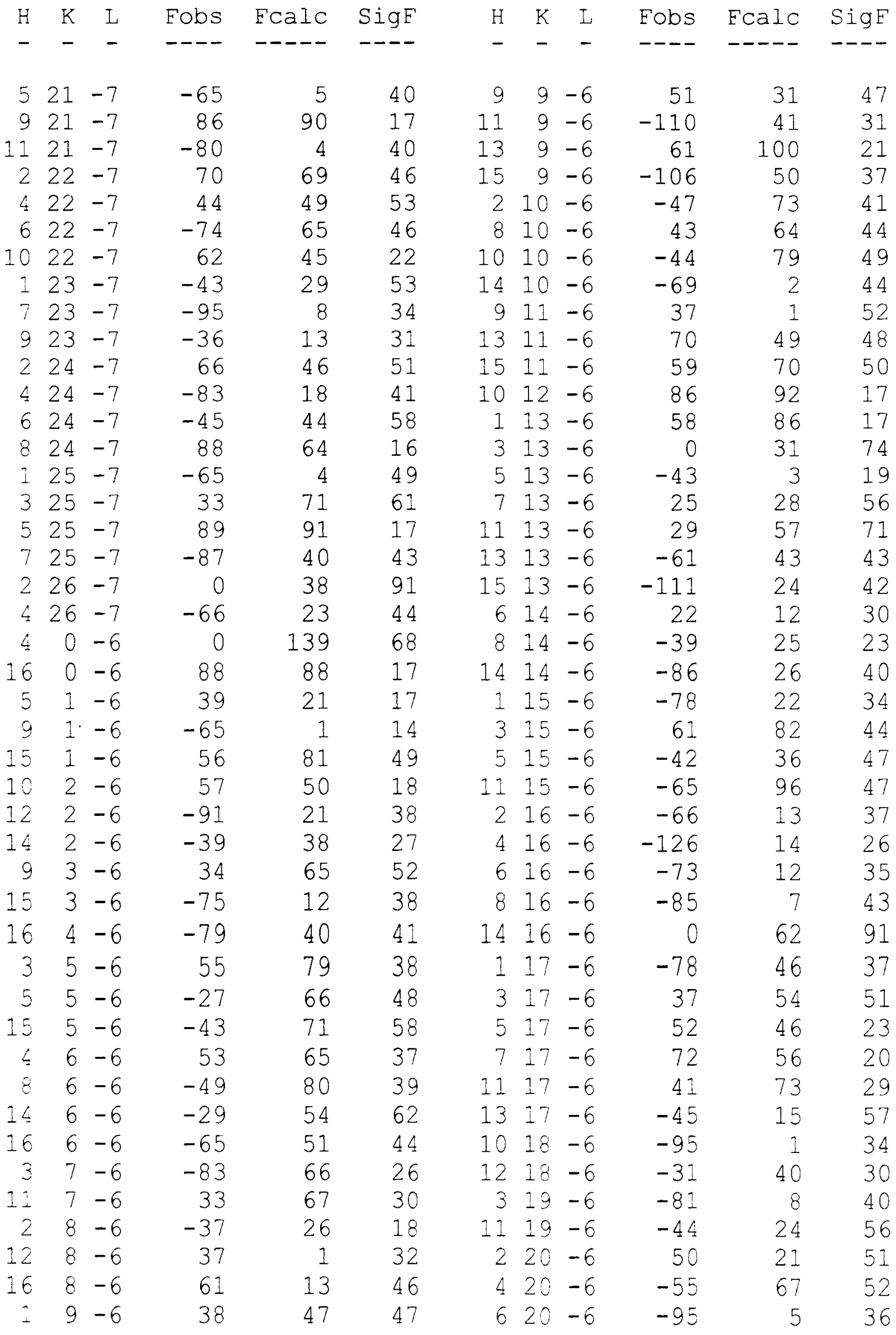


Reflexões não observadas $I<3 \sigma(I)$

Values of $10 *$ Fobs and 10*FCalc

Page 13

\begin{tabular}{|c|c|c|c|c|c|c|c|c|c|c|c|}
\hline $\mathrm{H}$ & K & $I$ & Eobs & Ecalc & SigF & $\mathrm{H}$ & K & L & Fobs & Ecalc & $\operatorname{Sig} F$ \\
\hline - & - & - & ---- & ---- & ---- & - & - & - & ---- & ----- & --- \\
\hline 82 & 0 & -6 & -41 & 41 & 57 & 16 & 4 & -5 & -56 & 22 & 54 \\
\hline 102 & 0 & -6 & 56 & 34 & 47 & 1 & 5 & -5 & 19 & 85 & 51 \\
\hline 122 & 0 & -6 & -66 & 8 & 48 & 15 & 5 & -5 & -77 & 8 & 41 \\
\hline 32 & 1 & -6 & -40 & 26 & 52 & 4 & 6 & -5 & 41 & 41 & 38 \\
\hline 52 & 1 & -6 & -50 & 32 & 48 & 6 & 6 & -5 & -39 & 35 & 18 \\
\hline 72 & 1 & -6 & 30 & 33 & 60 & 12 & 6 & -5 & 57 & 40 & 49 \\
\hline 92 & 1 & -6 & 45 & 86 & 57 & 16 & 6 & -5 & 46 & 55 & 29 \\
\hline 112 & 1 & -6 & 59 & 43 & 55 & 3 & 7 & -5 & 28 & 14 & 21 \\
\hline 62 & 2 & -6 & 86 & 104 & 16 & 5 & 7 & -5 & -33 & 120 & 45 \\
\hline 82 & 2 & -6 & -16 & 37 & 39 & 9 & 7 & -5 & -94 & 7 & 27 \\
\hline 102 & 2 & -6 & -65 & 57 & 41 & 10 & 8 & -5 & 86 & 120 & 14 \\
\hline 32 & 3 & -6 & -117 & 51 & 30 & 12 & 8 & -5 & 58 & 78 & 54 \\
\hline 72 & 3 & -6 & -43 & 21 & 51 & 14 & 8 & -5 & -91 & 139 & 49 \\
\hline 92 & 3 & -6 & 93 & 86 & 17 & 16 & 8 & -5 & -60 & 72 & 22 \\
\hline 22 & 4 & -6 & 45 & 59 & 57 & 1 & 9 & -5 & 58 & 93 & 13 \\
\hline 42 & 4 & -6 & -30 & 17 & 59 & 3 & 9 & -5 & 57 & 88 & 38 \\
\hline 62 & 4 & -6 & 65 & 62 & 49 & 11 & 9 & -5 & 28 & 12 & 67 \\
\hline 82 & 4 & -6 & 49 & 7 & 24 & 15 & 9 & -5 & 91 & 106 & 19 \\
\hline 12 & 5 & -6 & 25 & 30 & 35 & 12 & 10 & -5 & 66 & 82 & 23 \\
\hline 52 & 5 & -6 & 54 & 10 & 21 & 14 & 10 & -5 & 69 & 81 & 21 \\
\hline 72 & 5 & -6 & -27 & 14 & 34 & 5 & 11 & -5 & -52 & 42 & 36 \\
\hline 22 & 6 & -6 & -79 & 16 & 43 & 7 & 11 & -5 & 63 & 129 & 38 \\
\hline 42 & 6 & -6 & -86 & 30 & 37 & 9 & 11 & -5 & 42 & 57 & 22 \\
\hline 62 & 6 & -6 & -57 & 22 & 44 & 15 & 11 & -5 & 60 & 82 & 50 \\
\hline 12 & 7 & -6 & 39 & 13 & 30 & 6 & 12 & -5 & -64 & 74 & 35 \\
\hline 32 & $?$ & -6 & -33 & 16 & 63 & 10 & 12 & -5 & 27 & 91 & 74 \\
\hline 52 & 7 & -6 & 60 & 15 & 54 & 14 & 12 & -5 & -57 & 18 & 22 \\
\hline 2 & 0 & -5 & -53 & 0 & 35 & 1 & 13 & -5 & -31 & 17 & 44 \\
\hline 4 & 0 & -5 & -11 & 0 & 26 & 11 & 13 & -5 & 58 & 27 & 46 \\
\hline 6 & 0 & -5 & 0 & 0 & 67 & 13 & 13 & -5 & 30 & 24 & 33 \\
\hline 8 & 0 & -5 & 16 & 0 & 29 & 2 & 14 & -5 & 53 & 62 & 17 \\
\hline 10 & 0. & -5 & 50 & 0 & 41 & 4 & 14 & -5 & 58 & 65 & 35 \\
\hline 12 & 0 & -5 & -49 & 0 & 24 & 10 & 14 & -5 & 63 & 47 & 44 \\
\hline 14 & 0 & -5 & -125 & 0 & 28 & 14 & 14 & -5 & 48 & 1 & 61 \\
\hline 16 & 0 & -5 & 49 & 0 & 57 & 13 & 15 & -5 & -63 & 16 & 46 \\
\hline 5 & $i$ & -5 & 44 & 38 & 35 & 2 & 16 & -5 & 44 & 46 & 20 \\
\hline 7 & $\therefore$ & -5 & -38 & 85 & 38 & 12 & 16 & -5 & 92 & 73 & 15 \\
\hline 15 & 1 & -5 & -30 & 1 & 48 & 14 & 16 & -5 & 35 & 25 & 66 \\
\hline 6 & 2 & -5 & -46 & 62 & 15 & 7 & 17 & -5 & 66 & 31 & 19 \\
\hline 14 & 2 & -5 & -41 & 17 & 46 & 13 & 17 & -5 & 22 & 57 & 40 \\
\hline 16 & 2 & -5 & -65 & 5 & 39 & 2 & 18 & -5 & 59 & 54 & 38 \\
\hline 13 & 3 & -5 & 40 & 13 & 31 & 10 & 18 & -5 & -112 & 28 & 30 \\
\hline 15 & 3 & -5 & 93 & 100 & 17 & 12 & 18 & -5 & -45 & 1 & 56 \\
\hline 4 & 4 & -5 & 32 & 35 & 42 & 1 & 19 & -5 & 47 & 88 & 53 \\
\hline
\end{tabular}


Reflexões não observadas $I<3 \sigma(I)$

Values of $10 *$ Fobs and $10 * \mathrm{Fcalc}$

Page 14

\begin{tabular}{|c|c|c|c|c|c|c|c|c|c|c|}
\hline K & $I$ & Fobs & Fcalc & $\operatorname{Sig} F$ & $\mathrm{H}$ & K & $\mathrm{L}$ & Fobs & Fcalc & $\operatorname{Sig} F$ \\
\hline- & - & ---- & ----- & ---- & - & - & - & ---- & ----- & --- \\
\hline 319 & -5 & 72 & 15 & 16 & 6 & 10 & -4 & 65 & 59 & 13 \\
\hline 19 & -5 & -31 & 48 & 57 & 8 & 10 & -4 & -40 & 47 & 50 \\
\hline 420 & -5 & 86 & 122 & 18 & 14 & 10 & -4 & 80 & 85 & 19 \\
\hline 820 & -5 & 63 & 96 & 19 & 7 & 11 & -4 & 50 & 39 & 17 \\
\hline 20 & -5 & 80 & 67 & 17 & 11 & 11 & -4 & 0 & 73 & 96 \\
\hline 20 & -5 & 77 & 41 & 49 & 13 & 11 & -4 & 0 & 41 & 93 \\
\hline 121 & -5 & 62 & 21 & 23 & 8 & 12 & -4 & 66 & 36 & 39 \\
\hline 521 & -5 & 41 & 116 & 59 & 10 & 12 & -4 & -65 & 35 & 43 \\
\hline 721 & -5 & -41 & 15 & 52 & 12 & 12 & -4 & -34 & 60 & 26 \\
\hline 921 & -5 & -43 & 20 & 55 & 14 & 12 & -4 & 75 & 54 & 19 \\
\hline $\begin{array}{ll}1 & 21\end{array}$ & -5 & 59 & 75 & 53 & 3 & 13 & -4 & 49 & 65 & 36 \\
\hline 622 & -5 & 41 & 42 & 23 & 11 & 13 & -4 & 66 & 22 & 43 \\
\hline 822 & -5 & 38 & 46 & 29 & 13 & 13 & -4 & -43 & 44 & 57 \\
\hline 022 & -5 & -86 & 30 & 39 & 6 & 14 & -4 & -79 & 83 & 35 \\
\hline 323 & -5 & 53 & 72 & 48 & 14 & 14 & -4 & 69 & 56 & 21 \\
\hline 523 & -5 & -29 & 12 & 49 & 1 & 15 & -4 & -32 & 22 & 49 \\
\hline 723 & -5 & -135 & 9 & 30 & 3 & 15 & -4 & 37 & 2 & 20 \\
\hline 923 & -5 & 58 & 1 & 44 & 9 & 15 & -4 & -33 & 46 & 32 \\
\hline 424 & -5 & -43 & 18 & 56 & 13 & 15 & -4 & 74 & 69 & 52 \\
\hline 624 & -5 & -16 & 10 & 40 & 2 & 16 & -4 & 48 & 107 & 43 \\
\hline 824 & -5 & -91 & 51 & 41 & 10 & 16 & -4 & 34 & 47 & 29 \\
\hline 125 & -5 & -144 & 93 & 30 & 9 & 17 & -4 & 53 & 63 & 27 \\
\hline 525 & -5 & -54 & 13 & 50 & 13 & 17 & -4 & -32 & 81 & 67 \\
\hline 226 & -5 & -111 & 23 & 36 & 2 & 18 & -4 & -69 & 22 & 14 \\
\hline 426 & -5 & 47 & 83 & 59 & 10 & 18 & -4 & 0 & 56 & 53 \\
\hline 626 & -5 & -32 & 38 & 32 & 12 & 18 & -4 & -91 & 72 & 42 \\
\hline 127 & -5 & 97 & 117 & 17 & 1 & 19 & -4 & 68 & 83 & 19 \\
\hline 327 & -5 & 59 & 40 & 51 & 3 & 19 & -4 & 70 & 41 & 16 \\
\hline 27 & -5 & -81 & 34 & 40 & 10 & 20 & -4 & 0 & 9 & 80 \\
\hline 1 & -4 & -116 & 29 & 32 & 12 & 20 & -4 & -88 & 24 & 38 \\
\hline 1 & -4 & 41 & 48 & 28 & 7 & 21 & -4 & 79 & 81 & 16 \\
\hline 2 & -4 & 31 & 15 & 60 & 9 & 21 & -4 & 0 & 7 & 87 \\
\hline 3 & -4 & 30 & 74 & 65 & 11 & 21 & -4 & 68 & 3 & 44 \\
\hline 4 & -4 & 63 & 106 & 13 & 6 & 22 & -4 & 52 & 44 & 46 \\
\hline 4 & -4 & -57 & 85 & 49 & 8 & 22 & -4 & -92 & 22 & 35 \\
\hline 5 & -4 & 66 & 67 & 14 & 10 & 22 & -4 & 42 & 7 & 27 \\
\hline 6 & -4 & -54 & 40 & 39 & 1 & 23 & -4 & 60 & 22 & 37 \\
\hline 6 & -4 & -113 & 5 & 30 & 3 & 23 & -4 & 37 & 39 & 26 \\
\hline 6 & -4 & 12 & 70 & 45 & 7 & 23 & -4 & 63 & 67 & 46 \\
\hline 7 & -4 & -36 & 115 & 64 & 2 & 24 & -4 & 61 & 75 & 47 \\
\hline 7 & -4 & 34 & 5 & 59 & 4 & 24 & -4 & -90 & 9 & 33 \\
\hline 8 & -4 & 0 & 93 & 68 & 6 & 24 & -4 & -76 & 7 & 44 \\
\hline 9 & -4 & 31 & 104 & 65 & 8 & 24 & -4 & -72 & 22 & 40 \\
\hline 9 & -4 & 69 & 53 & 21 & 1 & 25 & -4 & -87 & 4 & 33 \\
\hline
\end{tabular}


Reflexões não observadas $I<3 \sigma$ (I)

Values of $10 *$ Fobs and 10*Fcalc

Page 15

\begin{tabular}{|c|c|c|c|c|c|c|c|c|c|c|c|}
\hline $\mathrm{H}$ & K & I & Fobs & Fcalc & SigE & $\mathrm{H}$ & K & $\mathrm{L}$ & Fobs & Fcalc & $\operatorname{Sig} E$ \\
\hline- & - & - & ---- & ---- & ---- & - & - & - & ---- & ----- & ---- \\
\hline 3 & 25 & -4 & -59 & 20 & 20 & 14 & 14 & -3 & -46 & 81 & 27 \\
\hline 5 & 25 & -4 & 32 & 24 & 61 & 13 & 15 & -3 & 75 & 127 & 51 \\
\hline 7 & 25 & -4 & 47 & 15 & 45 & 2 & 16 & -3 & 41 & 15 & 39 \\
\hline 4 & 26 & -4 & 43 & 16 & 27 & 10 & 16 & -3 & 42 & 84 & 48 \\
\hline 3 & 27 & -4 & 83 & 73 & 18 & 12 & 16 & -3 & -104 & 59 & 37 \\
\hline 2 & 28 & -4 & 59 & 16 & 45 & 11 & 17 & -3 & -87 & 22 & 39 \\
\hline 4 & 28 & -4 & -47 & 42 & 57 & 13 & 17 & -3 & -35 & 13 & 31 \\
\hline 2 & 0 & -3 & -33 & 0 & 16 & 2 & 18 & -3 & -93 & 10 & 30 \\
\hline 4 & 0 & -3 & -52 & 0 & 29 & 4 & 18 & -3 & -23 & 4 & 27 \\
\hline 6 & 0 & -3 & 42 & 0 & 36 & 1 & 19 & -3 & -25 & 75 & 62 \\
\hline 8 & 0 & -3 & -50 & 0 & 34 & 3 & 19 & -3 & 26 & 25 & 53 \\
\hline 10 & 0 & -3 & -75 & 0 & 30 & 9 & 19 & -3 & -146 & 15 & 23 \\
\hline 12 & 0 & -3 & -54 & 0 & 49 & 11 & 19 & -3 & 65 & 10 & 19 \\
\hline 14 & 0 & -3 & -95 & 0 & 35 & 2 & 20 & -3 & 66 & 56 & 42 \\
\hline 16 & 0 & -3 & -75 & 0 & 37 & 6 & 20 & -3 & 69 & 124 & 47 \\
\hline 9 & 1 & -3 & 33 & 2 & 47 & 8 & 20 & -3 & -41 & 59 & 50 \\
\hline 15 & 1 & -3 & 28 & 12 & 33 & 10 & 20 & -3 & 0 & 25 & 90 \\
\hline 5 & 3 & -3 & 6 & 9 & 31 & 3 & 21 & -3 & -38 & 20 & 50 \\
\hline 9 & 3 & -3 & -55 & 80 & 39 & 9 & 21 & -3 & -38 & 23 & 27 \\
\hline 15 & 3 & -3 & 34 & 60 & 68 & 11 & 21 & -3 & 84 & 84 & 18 \\
\hline 12 & 4 & -3 & -67 & 18 & 45 & 8 & 22 & -3 & 45 & 71 & 48 \\
\hline 7 & 5 & -3 & 52 & 24 & 32 & 10 & 22 & -3 & -61 & 13 & 21 \\
\hline 15 & 5 & -3 & 76 & 89 & 45 & 5 & 23 & -3 & -70 & 41 & 17 \\
\hline 8 & 6 & -3 & 60 & 39 & 14 & 7 & 23 & -3 & 74 & 66 & 18 \\
\hline 12 & 6 & -3 & -73 & 95 & 44 & 9 & 23 & -3 & -107 & 17 & 37 \\
\hline 14 & 6 & -3 & 65 & 104 & 47 & 2 & 24 & -3 & -72 & 29 & 41 \\
\hline 11 & 7 & -3 & 71 & 64 & 19 & 8 & 24 & -3 & 0 & 31 & 80 \\
\hline 12 & 8 & -3 & 49 & 78 & 28 & 1 & 25 & -3 & 66 & 104 & 21 \\
\hline 11 & 9 & -3 & 57 & 59 & 23 & 3 & 25 & -3 & 31 & 106 & 66 \\
\hline 13 & 9 & -3 & -19 & 4 & 35 & 5 & 25 & -3 & 45 & 15 & 46 \\
\hline 15 & 9 & -3 & -110 & 10 & 34 & 2 & 26 & -3 & 65 & 59 & 17 \\
\hline 6 & 10 & -3 & -20 & 70 & 53 & 1 & 27 & -3 & 66 & 67 & 50 \\
\hline 8 & 10 & -3 & -94 & 40 & 28 & 2 & 28 & -3 & 59 & 45 & 51 \\
\hline 12 & 10 & -3 & 58 & 78 & 19 & 4 & 28 & -3 & -33 & 97 & 65 \\
\hline 14 & 10 & -3 & 67 & 26 & 19 & 13 & 1 & -2 & -92 & 65 & 35 \\
\hline 9 & 11 & -3 & -75 & 52 & 34 & 15 & 1 & -2 & -32 & 37 & 66 \\
\hline 10 & 12 & -3 & -46 & 90 & 54 & 8 & 2 & -2 & 0 & 42 & 68 \\
\hline 12 & 12 & -3 & -89 & 36 & 35 & 10 & 2 & -2 & -37 & 60 & 21 \\
\hline 14 & 12 & -3 & 92 & 47 & 16 & 13 & 3 & -2 & -110 & 14 & 29 \\
\hline 7 & 13 & -3 & 74 & 212 & 43 & 14 & 4 & -2 & 65 & 77 & 46 \\
\hline 9 & 13 & -3 & 60 & 28 & 45 & 15 & 5 & -2 & 84 & 108 & 19 \\
\hline 13 & 13 & -3 & 70 & 14 & 19 & 6 & 6 & -2 & -50 & 56 & 32 \\
\hline 2 & 14 & -3 & 68 & 91 & 12 & 12 & 6 & -2 & -84 & 27 & 39 \\
\hline 12 & 14 & -3 & -96 & 23 & 31 & 14 & 6 & -2 & -31 & 45 & 54 \\
\hline
\end{tabular}


Reflexões não observadas $I<3 \sigma$ (I)

Values of $10 *$ Fobs and $10 *$ Fcalc

Page 16

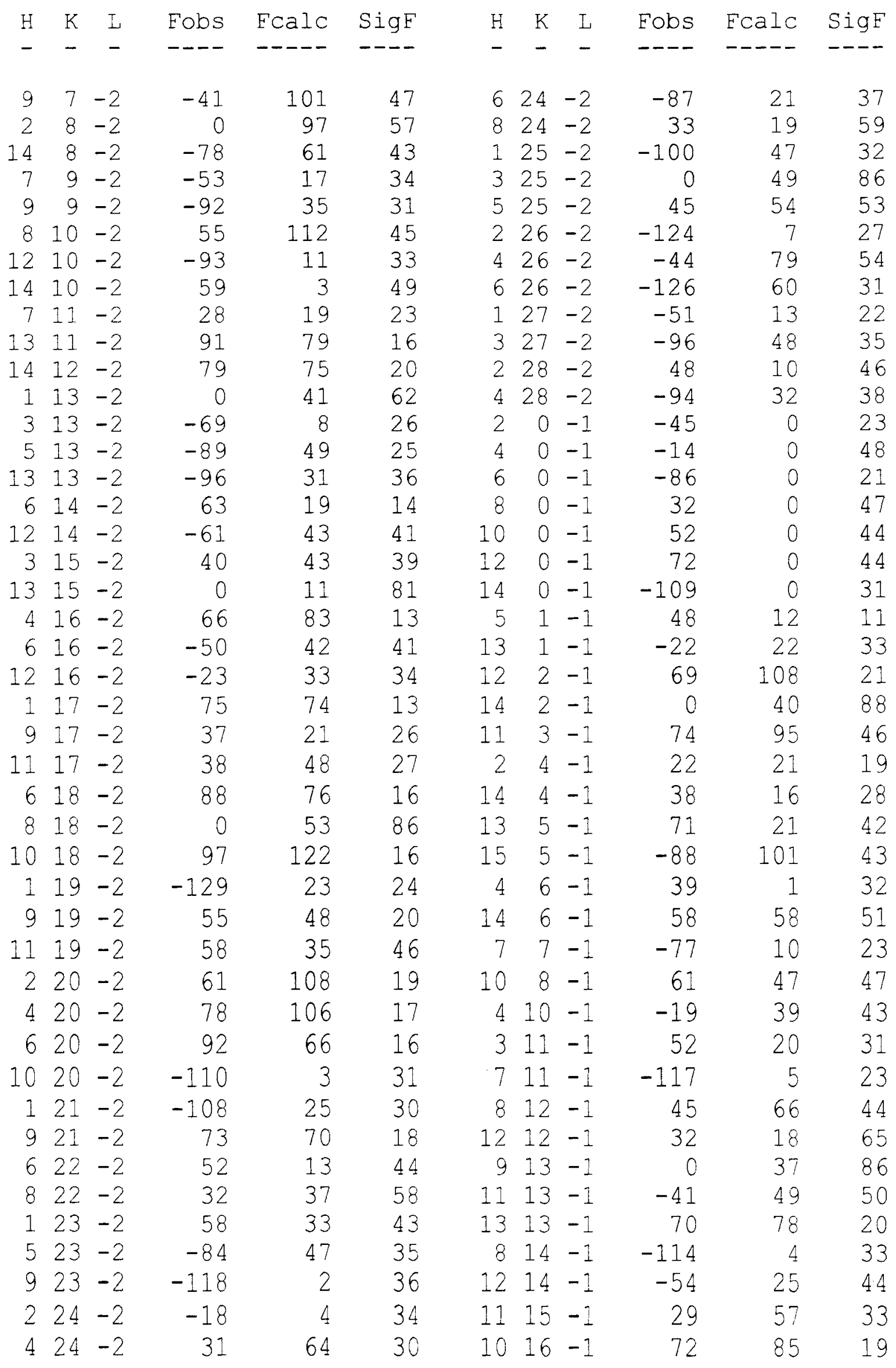


Reflexões não observadas $I<3 \sigma(I)$

Values of $10 *$ Fobs and $10 *$ Fcalc

Page 17

\begin{tabular}{|c|c|c|c|c|c|c|c|c|c|c|}
\hline $\mathrm{H}$ & L & Fobs & Fcalc & $\operatorname{Sig} F$ & $\mathrm{H}$ & K & $\mathrm{L}$ & Fobs & Fcalc & SigE \\
\hline - & - & ---- & ---- & ---- & - & - & - & ---- & ---- & ---- \\
\hline 1216 & -1 & -51 & 5 & 23 & 11 & 9 & 0 & 7 & 45 & 80 \\
\hline $5 \quad 17$ & -1 & 26 & 78 & 58 & 13 & 9 & 0 & 10 & 59 & 85 \\
\hline $8 \quad 18$ & -1 & -70 & 41 & 16 & 4 & 10 & 0 & 42 & 26 & 24 \\
\hline 1018 & -1 & 38 & 46 & 28 & 1 & 11 & 0 & 45 & 54 & 20 \\
\hline $12 \quad 18$ & -1 & -33 & 56 & 32 & 9 & 11 & 0 & 72 & 107 & 31 \\
\hline 119 & -1 & 26 & 49 & 57 & 0 & 12 & 0 & 44 & 41 & 14 \\
\hline 919 & -1 & 54 & 67 & 49 & 6 & 12 & 0 & 38 & 11 & 47 \\
\hline 220 & -1 & 32 & 56 & 30 & 10 & 12 & 0 & 95 & 124 & 17 \\
\hline 620 & -1 & -39 & 74 & 57 & 12 & 12 & 0 & 82 & 95 & 33 \\
\hline 1020 & -1 & -55 & 51 & 45 & 11 & 13 & 0 & 107 & 99 & 20 \\
\hline $\begin{array}{ll}5 & 21\end{array}$ & -1 & 29 & 20 & 64 & 13 & 13 & 0 & 70 & 102 & 36 \\
\hline 721 & -1 & 45 & 54 & 23 & 10 & 14 & 0 & -58 & 2 & 51 \\
\hline 622 & -1 & 61 & 68 & 20 & 12 & 14 & 0 & -39 & 34 & 55 \\
\hline 822 & -1 & 56 & 60 & 51 & 1 & 15 & 0 & 36 & 57 & 41 \\
\hline 1022 & -1 & -81 & 4 & 44 & 3 & 15 & 0 & 45 & 75 & 28 \\
\hline 323 & -1 & 11 & 7 & 41 & 8 & 16 & 0 & 36 & 27 & 59 \\
\hline $\begin{array}{ll}5 & 23\end{array}$ & -1 & 68 & 42 & 45 & 12 & 16 & 0 & 93 & 114 & 29 \\
\hline 723 & -1 & -30 & 12 & 61 & 11 & 17 & 0 & 79 & 106 & 67 \\
\hline 224 & -1 & -41 & 64 & 41 & 0 & 18 & 0 & 62 & 48 & 15 \\
\hline 424 & -1 & -95 & 18 & 34 & 8 & 18 & 0 & -26 & 67 & 31 \\
\hline 624 & -1 & -117 & 35 & 30 & 10 & 18 & 0 & 5 & 42 & 60 \\
\hline 125 & -1 & 0 & 116 & 88 & 1 & 19 & 0 & -83 & 29 & 39 \\
\hline 325 & -1 & 70 & 33 & 43 & 11 & 19 & 0 & 67 & 69 & 35 \\
\hline 725 & -1 & 75 & 119 & 50 & 8 & 20 & 0 & -46 & 17 & 39 \\
\hline 226 & -1 & 88 & 81 & 16 & 10 & 20 & 0 & -103 & 5 & 37 \\
\hline 426 & -1 & 58 & 59 & 21 & 3 & 21 & 0 & -29 & 26 & 49 \\
\hline 327 & -1 & 33 & 64 & 69 & 7 & 21 & 0 & -60 & 22 & 43 \\
\hline $\begin{array}{ll}5 & 27\end{array}$ & -1 & 77 & 72 & 18 & 9 & 21 & 0 & 45 & 33 & 40 \\
\hline 228 & -1 & 0 & 98 & 100 & 2 & 22 & 0 & -57 & 114 & 57 \\
\hline 428 & -1 & 66 & 12 & 19 & 4 & 22 & 0 & 36 & 27 & 49 \\
\hline 129 & -1 & -125 & 41 & 37 & 6 & 22 & 0 & -20 & 24 & 64 \\
\hline 11 & 0 & -61 & 20 & 46 & 8 & 22 & 0 & -21 & 6 & 66 \\
\hline 13 & 0 & 43 & 48 & 34 & 1 & 23 & 0 & 80 & 56 & 30 \\
\hline 12 & 0 & -89 & 18 & 34 & 5 & 23 & 0 & 25 & 48 & 49 \\
\hline 13 & 0 & 98 & 90 & 34 & 7 & 23 & 0 & -74 & 62 & 40 \\
\hline 12 & 0 & 74 & 80 & 29 & 0 & 24 & 0 & -28 & 94 & 55 \\
\hline 14 & 0 & 53 & 80 & 68 & 2 & 24 & 0 & 65 & 36 & 32 \\
\hline 5 & 0 & 30 & 27 & 42 & 4 & 24 & 0 & 33 & 28 & 37 \\
\hline 10 & 50 & -29 & 34 & 45 & 6 & 24 & 0 & -58 & 41 & 42 \\
\hline 12 & 50 & -41 & 48 & 52 & 8 & 24 & 0 & -46 & 48 & 65 \\
\hline 3 & 0 & 53 & 52 & 19 & 1 & 25 & 0 & 44 & 36 & 49 \\
\hline 13 & 0 & -28 & 37 & 38 & 3 & 25 & 0 & -75 & 36 & 38 \\
\hline 14 & 0 & 15 & 18 & 54 & 5 & 25 & 0 & -31 & 43 & 62 \\
\hline 7 & 0 & 13 & 36 & 41 & 7 & 25 & 0 & 69 & 59 & 33 \\
\hline
\end{tabular}


Reflexões não observadas $I<3 \sigma$ (I)

Values of $10 *$ Fobs and $10 *$ Ecalc

Page 18

\begin{tabular}{|c|c|c|c|c|c|c|c|c|c|c|c|}
\hline $\mathrm{H}$ & K & L & Fobs & Fcalc & SigF & $\mathrm{H}$ & K & L & Eobs & Fcalc & $\operatorname{Sig} F$ \\
\hline- & - & - & ---- & ----- & ---- & - & - & - & ---- & - --ー- & ---- \\
\hline 2 & 26 & 0 & 65 & 24 & 32 & 0 & 18 & 1 & -34 & 59 & 49 \\
\hline 6 & 26 & 0 & 63 & 45 & 35 & 1 & 19 & 1 & -62 & 20 & 39 \\
\hline 3 & 27 & 0 & 106 & 104 & 35 & 7 & 19 & 1 & 30 & 1 & 53 \\
\hline 0 & 28 & 0 & 67 & 23 & 41 & 9 & 19 & 1 & 61 & 56 & 20 \\
\hline 4 & 28 & 0 & -42 & 4 & 42 & 6 & 20 & 1 & -58 & 40 & 41 \\
\hline 1 & 29 & 0 & -57 & 18 & 45 & 8 & 20 & 1 & 56 & 22 & 51 \\
\hline 2 & 0 & 1 & -73 & 0 & 22 & 10 & 20 & 1 & 26 & 77 & 37 \\
\hline 4 & 0 & 1 & -59 & 0 & 25 & 1 & 21 & 1 & 63 & 11 & 18 \\
\hline 6 & 0 & 1 & -52 & 0 & 28 & 5 & 21 & 1 & 60 & 95 & 47 \\
\hline 8 & 0 & 1 & 54 & 0 & 38 & 7 & 21 & 1 & -68 & 30 & 38 \\
\hline 10 & 0 & 1 & -88 & 0 & 37 & 6 & 22 & 1 & 77 & 81 & 17 \\
\hline 12 & 0 & 1 & 35 & 0 & 26 & 8 & 22 & 1 & -97 & 3 & 35 \\
\hline 14 & 0 & 1 & 35 & 0 & 66 & 3 & 23 & 1 & 0 & 58 & 82 \\
\hline 13 & 1 & 1 & 66 & 40 & 42 & 7 & 23 & 1 & 33 & 31 & 63 \\
\hline 8 & 2 & 1 & 50 & 57 & 17 & 0 & 24 & 1 & 60 & 48 & 17 \\
\hline 14 & 4 & 1 & 78 & 108 & 50 & 6 & 24 & 1 & -29 & 2 & 30 \\
\hline 9 & 5 & 1 & -42 & 20 & 21 & 3 & 25 & 1 & -69 & 22 & 40 \\
\hline 13 & 5 & 1 & 75 & 64 & 46 & 0 & 26 & 1 & 55 & 65 & 53 \\
\hline 9 & 7 & 1 & -39 & 3 & 23 & 2 & 26 & 1 & 49 & 77 & 25 \\
\hline 8 & 8 & 1 & -49 & 27 & 43 & 5 & 27 & 1 & 49 & 101 & 60 \\
\hline 10 & 8 & 1 & 17 & 28 & 44 & 11 & $I$ & 2 & -71 & 31 & 16 \\
\hline 5 & 9 & 1 & -47 & 9 & 34 & 13 & 1 & 2 & 68 & 1 & 46 \\
\hline 9 & 9 & 1 & -45 & 9 & 51 & 6 & 2 & 2 & 55 & 65 & 13 \\
\hline 13 & 9 & 1 & -36 & 27 & 28 & 8 & 2 & 2 & 62 & 30 & 37 \\
\hline 6 & 10 & 1 & 51 & 64 & 17 & 11 & 3 & 2 & 53 & 20 & 50 \\
\hline 12 & 10 & 1 & -60 & 46 & 19 & 13 & 3 & 2 & 37 & 65 & 31 \\
\hline 13 & 11 & 1 & -88 & 61 & 40 & 12 & 4 & 2 & 74 & 45 & 18 \\
\hline 6 & 12 & 1 & 35 & 56 & 52 & 11 & 5 & 2 & 62 & 146 & 51 \\
\hline 8 & 12 & 1 & -95 & 19 & 31 & 4 & 6 & 2 & -57 & 40 & 29 \\
\hline 10 & 12 & 1 & -65 & 63 & 42 & 10 & 6 & 2 & -27 & 27 & 34 \\
\hline 12 & 12 & 1 & 48 & 56 & 51 & 12 & 6 & 2 & -70 & 59 & 37 \\
\hline 5 & 13 & 1 & -46 & 4 & 43 & 8 & 8 & 2 & -57 & 0 & 36 \\
\hline 7 & 13 & 1 & -51 & 75 & 41 & 12 & 8 & 2 & 36 & 94 & 30 \\
\hline 11 & 13 & 1 & -98 & 51 & 37 & 9 & 9 & 2 & 57 & 52 & 50 \\
\hline 0 & 14 & 1 & -36 & 65 & 40 & 11 & 9 & 2 & -74 & 77 & 37 \\
\hline 2 & 14 & 1 & 50 & 68 & 37 & 2 & 10 & 2 & 59 & 82 & 12 \\
\hline 10 & 14 & 1 & -60 & 1 & 44 & 4 & 10 & 2 & 32 & 31 & 41 \\
\hline 12 & 14 & 1 & -65 & 105 & 51 & 8 & 10 & 2 & 33 & 68 & 30 \\
\hline 2 & 16 & 1 & -56 & 36 & 37 & 10 & 10 & 2 & 0 & 61 & 84 \\
\hline 8 & 16 & 1 & -69 & 67 & 36 & 12 & 10 & 2 & -64 & 6 & 45 \\
\hline 10 & 16 & 1 & 65 & 39 & 44 & 5 & 11 & 2 & 59 & 110 & 39 \\
\hline 7 & 17 & 1 & -88 & 108 & 38 & 9 & 11 & 2 & 49 & 73 & 28 \\
\hline 9 & 17 & 1 & -42 & 24 & 57 & 11 & 11 & 2 & 65 & 33 & 20 \\
\hline 1 & 17 & 1 & 0 & 47 & 92 & 4 & 12 & 2 & -50 & 7 & 40 \\
\hline
\end{tabular}


Reflexões não observadas $I<3 \sigma$ (I)

Values of $10 *$ Fobs and $10 *$ Ecalc

Page 19

\begin{tabular}{|c|c|c|c|c|c|c|c|c|c|c|}
\hline K & I & Fobs & Ecalc & $\operatorname{SigF}$ & $\mathrm{H}$ & K & L & Fobs & Fcalc & SigF \\
\hline - & - & ---- & ---- & ---- & - & - & - & ---- & ----- & --- \\
\hline 313 & 2 & 66 & 15 & 12 & 12 & 0 & 3 & 66 & 0 & 45 \\
\hline 713 & 2 & -26 & 72 & 66 & 11 & 1 & 3 & -42 & 29 & 47 \\
\hline 913 & 2 & 58 & 68 & 20 & 12 & 2 & 3 & -44 & 60 & 50 \\
\hline 1113 & 2 & -55 & 40 & 24 & 11 & 3 & 3 & -47 & 75 & 25 \\
\hline 1014 & 2 & -75 & 130 & 41 & 10 & 4 & 3 & -49 & 45 & 44 \\
\hline 115 & 2 & 46 & 6 & 35 & 12 & 4 & 3 & -78 & 34 & 39 \\
\hline 715 & 2 & 63 & 74 & 23 & 5 & 5 & 3 & 50 & 41 & 36 \\
\hline 1115 & 2 & -73 & 23 & 46 & 11 & 5 & 3 & 0 & 5 & 81 \\
\hline 616 & 2 & -46 & 31 & 56 & 12 & 6 & 3 & 34 & 0 & 62 \\
\hline 816 & 2 & 0 & 114 & 89 & 5 & 7 & 3 & 46 & 60 & 41 \\
\hline 1016 & 2 & -31 & 24 & 60 & 8 & 8 & 3 & 39 & 25 & 26 \\
\hline $7 \quad 17$ & 2 & 52 & 44 & 45 & 12 & 8 & 3 & 64 & 127 & 23 \\
\hline 917 & 2 & -53 & 68 & 51 & 4 & 10 & 3 & 52 & 10 & 16 \\
\hline 618 & 2 & -130 & 18 & 29 & 12 & 10 & 3 & 99 & 93 & 18 \\
\hline 1018 & 2 & 81 & 72 & 18 & 11 & 11 & 3 & 80 & 85 & 19 \\
\hline 919 & 2 & -96 & 39 & 33 & 10 & 12 & 3 & 0 & 6 & 82 \\
\hline 020 & 2 & -94 & 82 & 35 & 7 & 13 & 3 & 57 & 55 & 23 \\
\hline 420 & 2 & -79 & 42 & 38 & 9 & 13 & 3 & 31 & 29 & 64 \\
\hline 620 & 2 & 70 & 87 & 19 & 11 & 13 & 3 & -46 & 56 & 60 \\
\hline 820 & 2 & -83 & 17 & 36 & 0 & 14 & 3 & 32 & 30 & 21 \\
\hline $\begin{array}{ll}7 & 21\end{array}$ & 2 & 43 & 56 & 26 & 6 & 14 & 3 & -46 & 72 & 54 \\
\hline 921 & 2 & 91 & 125 & 18 & 10 & 14 & 3 & 58 & 71 & 51 \\
\hline 222 & 2 & 85 & 126 & 17 & 7 & 15 & 3 & -69 & 89 & 45 \\
\hline 422 & 2 & 31 & 28 & 60 & 9 & 15 & 3 & 0 & 8 & 84 \\
\hline 622 & 2 & 34 & 6 & 28 & 2 & 16 & 3 & -100 & 21 & 25 \\
\hline 323 & 2 & 60 & 72 & 20 & 10 & 16 & 3 & -56 & 38 & 52 \\
\hline 523 & 2 & 32 & 104 & 71 & 9 & 17 & 3 & -64 & 72 & 20 \\
\hline 723 & 2 & -32 & 19 & 66 & 6 & 18 & 3 & -109 & 5 & 29 \\
\hline 024 & 2 & -63 & 20 & 18 & 8 & 18 & 3 & 46 & 39 & 50 \\
\hline 224 & 2 & -67 & 45 & 46 & 5 & 19 & 3 & 66 & 75 & 18 \\
\hline 424 & 2 & -61 & 7 & 45 & 7 & 19 & 3 & 0 & 75 & 84 \\
\hline 624 & 2 & -66 & 33 & 21 & 9 & 19 & 3 & -27 & 55 & 34 \\
\hline 125 & 2 & 31 & 20 & 58 & 0 & 20 & 3 & -36 & 34 & 26 \\
\hline 325 & 2 & 77 & 76 & 18 & 4 & 20 & 3 & -90 & 27 & 35 \\
\hline 026 & 2 & -103 & 18 & 35 & 8 & 20 & 3 & 48 & 19 & 57 \\
\hline 426 & 2 & 64 & 36 & 20 & 3 & 21 & 3 & -90 & 10 & 40 \\
\hline 127 & 2 & -64 & 28 & 38 & 5 & 21 & 3 & 86 & 89 & 15 \\
\hline 028 & 2 & -27 & 12 & 33 & 7 & 21 & 3 & 67 & 105 & 55 \\
\hline 00 & 3 & 50 & 0 & 12 & 2 & 22 & 3 & -40 & 64 & 47 \\
\hline 20 & 3 & 48 & 0 & 12 & 1 & 23 & 3 & 55 & 37 & 21 \\
\hline 40 & 3 & 35 & 0 & 37 & 3 & 23 & 3 & -79 & 35 & 36 \\
\hline 60 & 3 & -58 & 0 & 29 & 5 & 23 & 3 & -31 & 17 & 60 \\
\hline 0 & 3 & -67 & 0 & 35 & 0 & 24 & 3 & -41 & 98 & 54 \\
\hline 00 & 3 & 0 & 0 & 82 & 2 & 24 & 3 & 64 & 60 & 20 \\
\hline
\end{tabular}


Reflexões não observadas $I<3 \sigma$ (I)

Values of $10 *$ Fobs and $10 *$ Fcalc

Page 20

\begin{tabular}{|c|c|c|c|c|c|c|c|c|c|c|c|}
\hline $\mathrm{H}$ & $\mathrm{K}$ & L & Fobs & Fcalc & SigF & $\mathrm{H}$ & K & I & Eobs & Fcalc & $\operatorname{SigF}$ \\
\hline . & - & - & ---- & ---- & --- & - & - & - & --- & ---- & - \\
\hline 4 & 24 & 3 & 33 & 2 & 65 & 9 & 17 & 4 & 69 & 51 & 47 \\
\hline 6 & 24 & 3 & 60 & 104 & 48 & 8 & 18 & 4 & 60 & 5 & 22 \\
\hline 1 & 25 & 3 & 44 & 13 & 25 & 7 & 19 & 4 & 0 & 61 & 89 \\
\hline 5 & 25 & 3 & 69 & 115 & 57 & 6 & 20 & 4 & 33 & 11 & 60 \\
\hline 0 & 26 & 3 & 32 & 49 & 66 & 8 & 20 & 4 & -33 & 13 & 60 \\
\hline 2 & 26 & 3 & 82 & 42 & 16 & 1 & 21 & 4 & 76 & 77 & 20 \\
\hline 1 & 27 & 3 & 34 & 54 & 66 & 3 & 21 & 4 & 82 & 101 & 17 \\
\hline 3 & 27 & 3 & 64 & 67 & 22 & 5 & 21 & 4 & 0 & 14 & 54 \\
\hline 0 & 28 & 3 & 92 & 68 & 16 & 7 & 21 & 4 & 89 & 94 & 17 \\
\hline 12 & 0 & 4 & -46 & 23 & 54 & 0 & 22 & 4 & 66 & 53 & 18 \\
\hline 9 & 1 & 4 & -79 & 105 & 41 & 2 & 22 & 4 & 0 & 11 & 50 \\
\hline-1 & 1 & 4 & 0 & 55 & 83 & 4 & 22 & 4 & 45 & 44 & 51 \\
\hline 4 & 2 & 4 & -71 & 63 & 29 & 6 & 22 & 4 & -45 & 1 & 53 \\
\hline 3 & 3 & 4 & 43 & 69 & 16 & 3 & 23 & 4 & 32 & 62 & 59 \\
\hline 11 & 3 & 4 & 69 & 55 & 19 & 5 & 23 & 4 & 34 & 43 & 30 \\
\hline 2 & 4 & 4 & 56 & 44 & 13 & 0 & 24 & 4 & -90 & 76 & 35 \\
\hline 6 & 4 & 4 & 43 & 34 & 47 & 2 & 24 & 4 & -82 & 27 & 35 \\
\hline 10 & 4 & 4 & -66 & 4 & 41 & 4 & 24 & 4 & 0 & 15 & 85 \\
\hline 12 & 4 & 4 & 71 & 106 & 24 & 1 & 25 & 4 & -31 & 25 & 63 \\
\hline 4 & 6 & 4 & 53 & 59 & 15 & 3 & 25 & 4 & -121 & 29 & 31 \\
\hline 8 & 6 & 4 & 74 & 54 & 19 & 0 & 26 & 4 & 39 & 38 & 28 \\
\hline 10 & 6 & 4 & 74 & 59 & 19 & 2 & 26 & 4 & 59 & 76 & 47 \\
\hline 7 & 7 & 4 & -25 & 102 & 56 & 1 & 27 & 4 & -73 & 89 & 46 \\
\hline 11 & 7 & 4 & 38 & 37 & 30 & 0 & 0 & 5 & -62 & 0 & 27 \\
\hline 2 & 8 & 4 & -28 & 13 & 45 & 2 & 0 & 5 & 53 & 0 & 33 \\
\hline 10 & 8 & 4 & 92 & 108 & 17 & 4 & 0 & - & -53 & 0 & 35 \\
\hline 5 & 9 & 4 & 55 & 45 & 17 & 6 & 0 & 5 & -24 & 0 & 56 \\
\hline 7 & 9 & 4 & -65 & 24 & 41 & 8 & 0 & 5 & 29 & 0 & 65 \\
\hline 11 & 9 & 4 & 59 & 12 & 45 & 10 & 0 & 5 & 0 & 0 & 51 \\
\hline 4 & 10 & 4 & -77 & 28 & 34 & 3 & 1 & 5 & -29 & 63 & 42 \\
\hline 1 & 11 & 4 & -79 & 6 & 24 & 5 & 1 & 5 & 19 & 67 & 30 \\
\hline 3 & 11 & 4 & 63 & 103 & 38 & 11 & 1 & 5 & 48 & 6 & 25 \\
\hline 7 & 11 & 4 & -91 & 70 & 36 & 2 & 2 & 5 & 54 & 33 & 36 \\
\hline 9 & 11 & 4 & 91 & 86 & 16 & 4 & 2 & 5 & 40 & 50 & 42 \\
\hline 2 & 12 & 4 & 47 & 45 & 40 & 8 & 2 & 5 & -56 & 25 & 24 \\
\hline 6 & 12 & 4 & 55 & 40 & 51 & 10 & 2 & $\bar{\Sigma}$ & 46 & 71 & 56 \\
\hline 10 & 12 & 4 & -60 & 48 & 21 & 5 & 3 & $\overline{5}$ & 39 & 6 & 20 \\
\hline 1 & 13 & 4 & -44 & 14 & 39 & 11 & 3 & 5 & 34 & 94 & 69 \\
\hline 8 & 14 & 4 & 45 & 37 & 55 & 0 & 4 & 5 & -86 & 24 & 24 \\
\hline 10 & 14 & 4 & 23 & 14 & 38 & 8 & 4 & $\vdots$ & -84 & 47 & 43 \\
\hline 5 & 15 & 4 & 0 & 135 & 91 & 7 & 5 & 5 & -84 & 17 & 38 \\
\hline 7 & 15 & 4 & -29 & 102 & 63 & 11 & 5 & 5 & 97 & 69 & 17 \\
\hline 9 & 15 & 4 & 70 & 88 & 21 & 8 & 6 & 5 & 44 & 79 & 31 \\
\hline 6 & 16 & 4 & 30 & 79 & 59 & 10 & 6 & $\Xi$ & 0 & 80 & 88 \\
\hline
\end{tabular}


Reflexões não observadas I < $3 \sigma(I)$

Values of $10 *$ Eobs and 10*Fcalc

Page 21

\begin{tabular}{|c|c|c|c|c|c|c|c|c|c|c|c|}
\hline $\mathrm{H}$ & K & L & Eobs & Fcalc & SigF & $\mathrm{H}$ & K & I & Eobs & Ecalc & SigF \\
\hline- & - & - & ---- & ---- & ---- & - & - & - & ---- & ---- & ---- \\
\hline 7 & 7 & 5 & -98 & 28 & 35 & 3 & 25 & 5 & 60 & 93 & 50 \\
\hline 8 & 8 & 5 & 56 & 93 & 23 & 0 & 26 & 5 & 67 & 56 & 47 \\
\hline 10 & 8 & 5 & 75 & 91 & 46 & 4 & 0 & 6 & -24 & 56 & 27 \\
\hline 5 & 9 & 5 & -98 & 65 & 27 & 3 & 1 & 6 & 75 & 63 & 13 \\
\hline 7 & 9 & 5 & 58 & 14 & 52 & 5 & 1 & 6 & -34 & 6 & 47 \\
\hline 9 & 9 & 5 & 64 & 101 & 21 & 9 & 1 & 6 & -81 & 16 & 38 \\
\hline 4 & 10 & 5 & -8 & 33 & 38 & 2 & 2 & 6 & -49 & 34 & 15 \\
\hline 6 & 10 & 5 & 87 & 84 & 16 & 4 & 2 & 6 & 55 & 7 & 39 \\
\hline 8 & 10 & 5 & -66 & 1 & 42 & 6 & 2 & 6 & -53 & 204 & 23 \\
\hline 10 & 10 & 5 & -64 & 19 & 51 & 9 & 3 & 6 & -28 & 21 & 31 \\
\hline 5 & 11 & 5 & 67 & 67 & 44 & 10 & 4 & 6 & -112 & 22 & 37 \\
\hline 9 & 11 & 5 & 47 & 76 & 50 & 5 & 5 & 6 & -62 & 86 & 42 \\
\hline 4 & 12 & 5 & -50 & 50 & 43 & 9 & 5 & 6 & 71 & 56 & 19 \\
\hline 6 & 12 & 5 & -46 & 1 & 26 & 2 & 6 & 6 & 37 & 16 & 22 \\
\hline 8 & 12 & 5 & -34 & 22 & 27 & 8 & 6 & 6 & -27 & 38 & 30 \\
\hline 10 & 12 & 5 & 64 & 30 & 23 & 10 & 6 & 6 & 49 & 52 & 52 \\
\hline 3 & 13 & 5 & -66 & 51 & 15 & 5 & 7 & 6 & 66 & 44 & 16 \\
\hline 5 & 13 & 5 & 40 & 27 & 57 & 7 & 7 & 6 & 67 & 124 & 47 \\
\hline 9 & 13 & 5 & -34 & 8 & 30 & 9 & 7 & 6 & 91 & 102 & 17 \\
\hline 0 & 14 & 5 & -53 & 32 & 17 & 6 & 8 & 6 & 49 & 54 & 49 \\
\hline 2 & 14 & 5 & -31 & 11 & 25 & 10 & 8 & 6 & 70 & 69 & 45 \\
\hline 6 & 14 & 5 & 34 & 44 & 29 & 7 & 9 & 6 & -40 & 6 & 55 \\
\hline 8 & 14 & 5 & -136 & 24 & 31 & 9 & 9 & 6 & 68 & 73 & 20 \\
\hline 0 & 16 & 5 & 51 & 107 & 50 & 0 & 10 & 6 & -38 & 15 & 42 \\
\hline 6 & 16 & 5 & 44 & 99 & 54 & 2 & 10 & 6 & -33 & 9 & 53 \\
\hline 8 & 16 & 5 & -71 & 37 & 35 & 6 & 10 & 6 & 41 & 38 & 60 \\
\hline 5 & 17 & 5 & 89 & 84 & 16 & 8 & 10 & 6 & 72 & 32 & 43 \\
\hline 7 & 17 & 5 & 41 & 35 & 27 & 1 & 11 & 6 & 55 & 11 & 44 \\
\hline 6 & 18 & 5 & -92 & 104 & 37 & 3 & 11 & 6 & -23 & 3 & 30 \\
\hline 8 & 18 & 5 & 93 & 115 & 19 & 7 & 11 & 6 & 63 & 30 & 42 \\
\hline 5 & 19 & 5 & -60 & 8 & 43 & 9 & 11 & 6 & -122 & 31 & 31 \\
\hline 7 & 19 & 5 & 68 & 77 & 48 & 2 & 12 & 6 & 0 & 0 & 80 \\
\hline 2 & 20 & 5 & 68 & 27 & 16 & 1 & 13 & 6 & -48 & 11 & 42 \\
\hline 4 & 20 & 5 & 55 & 11 & 48 & 5 & 13 & 6 & 72 & 34 & 44 \\
\hline 6 & 20 & 5 & 52 & 24 & 24 & 7 & 13 & 6 & -43 & 37 & 56 \\
\hline 3 & 21 & 5 & 31 & 52 & 65 & 9 & 13 & 6 & -105 & 22 & 37 \\
\hline 5 & 21 & 5 & -123 & 22 & 32 & 0 & 14 & 6 & -42 & 55 & 47 \\
\hline 0 & 22 & 5 & 61 & 12 & 19 & 2 & 14 & 6 & 27 & 34 & 55 \\
\hline 4 & 22 & 5 & 22 & 44 & 38 & 8 & 14 & 6 & 59 & 105 & 24 \\
\hline 6 & 22 & 5 & 0 & 7 & 88 & 5 & 15 & 6 & -82 & 8 & 33 \\
\hline 1 & 23 & 5 & -105 & 53 & 33 & 4 & 16 & 6 & -57 & 56 & 45 \\
\hline 5 & 23 & 5 & -24 & 52 & 39 & 6 & 16 & 6 & 66 & 88 & 20 \\
\hline 4 & 24 & 5 & -93 & 5 & 34 & 8 & 16 & 6 & -57 & 8 & 44 \\
\hline 1 & 25 & 5 & 59 & 36 & 20 & 5 & 17 & 6 & 45 & 98 & 53 \\
\hline
\end{tabular}


Reflexões não observadas $I<3 \sigma(I)$

Values of $10 *$ Fobs and $10 *$ Fcalc

Page 22

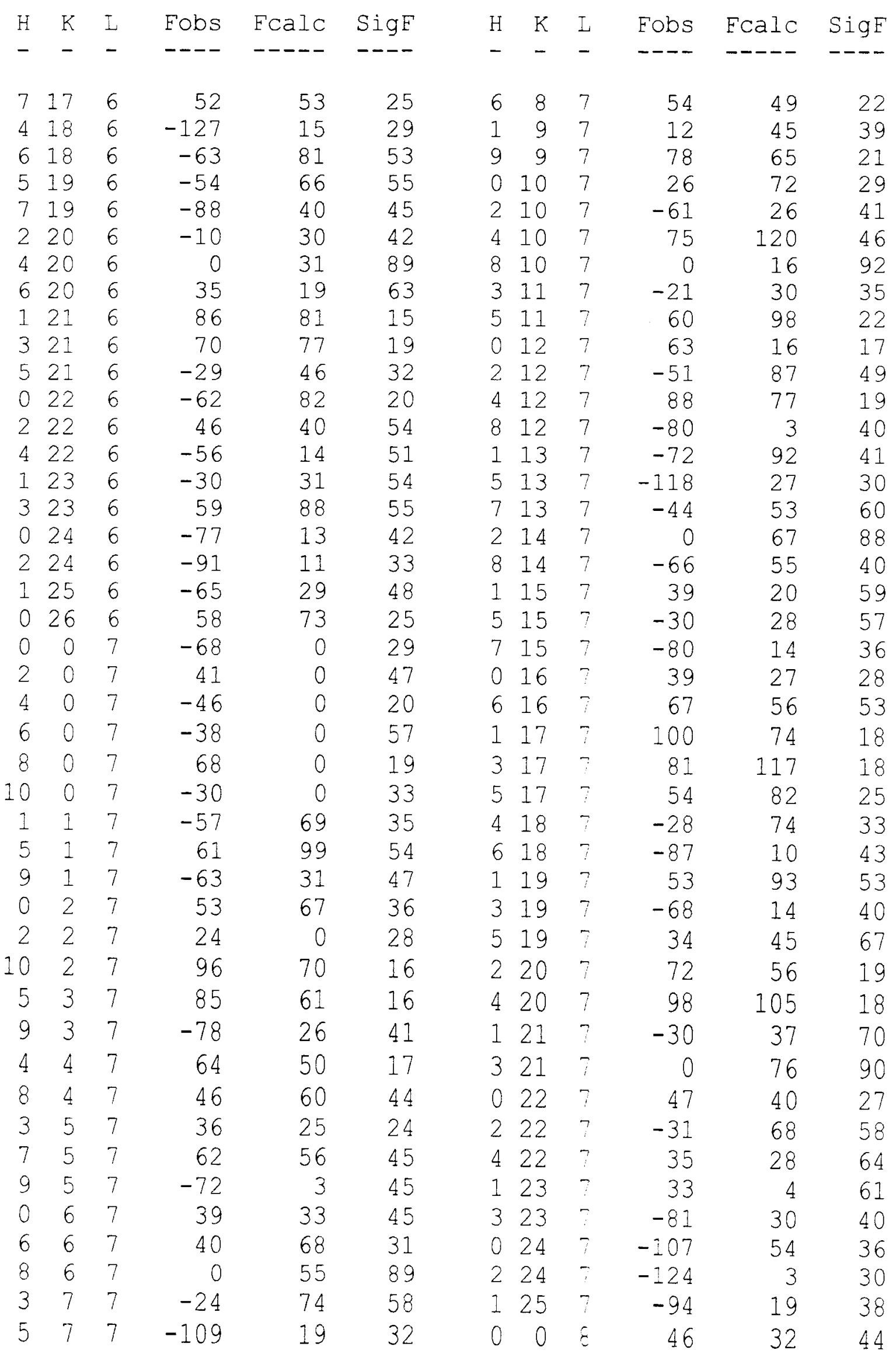


Reflexões não observadas $I<3 \sigma(I)$

Values of $10 *$ Fobs and $10 *$ Ecalc

Page 23

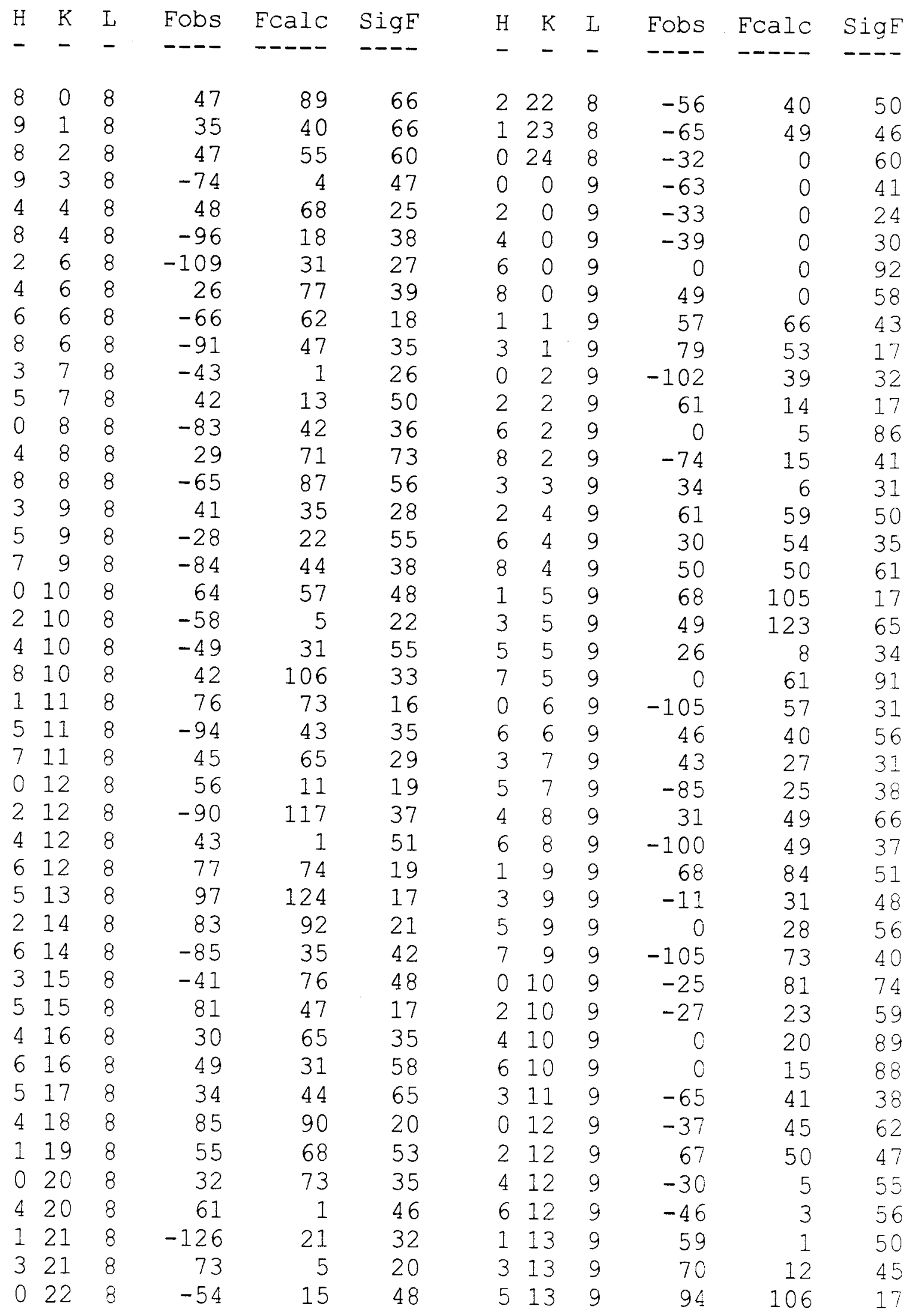


Reflexões não observadas $I<3 \sigma(I)$

Values of $10 *$ Fobs and $10 *$ Fcalc

Page 24

\begin{tabular}{|c|c|c|c|c|c|c|c|c|c|c|}
\hline $\mathrm{H}$ & L & Eobs & Ecalc & SigF & $\mathrm{H}$ & $\mathrm{K}$ & I & Fobs & Ecalc & SigF \\
\hline - & - & ---- & ----- & ---- & - & - & - & ---- & ----- & $-\cdots$ \\
\hline 014 & 9 & 58 & 22 & 52 & 3 & 11 & 10 & 79 & 82 & 48 \\
\hline 414 & 9 & 0 & 38 & 90 & 5 & 11 & 10 & -73 & 4 & 44 \\
\hline 515 & 9 & -24 & 50 & 38 & 0 & 12 & 10 & -28 & 41 & 38 \\
\hline 216 & 9 & -30 & 88 & 74 & 2 & 12 & 10 & -29 & 23 & 66 \\
\hline 416 & 9 & 59 & 76 & 50 & 1 & 13 & 10 & 0 & 63 & 90 \\
\hline 317 & 9 & 77 & 52 & 19 & 3 & 13 & 10 & 68 & 40 & 23 \\
\hline 418 & 9 & -24 & 27 & 37 & 5 & 13 & 10 & -125 & 17 & 34 \\
\hline 119 & 9 & 66 & 71 & 23 & 0 & 14 & 10 & -45 & 15 & 26 \\
\hline 319 & 9 & -114 & 7 & 34 & 3 & 15 & 10 & 59 & 13 & 57 \\
\hline 020 & 9 & -122 & 49 & 35 & 2 & 16 & 10 & 75 & 70 & 48 \\
\hline 220 & 9 & 60 & 41 & 52 & 3 & 17 & 10 & -46 & 58 & 60 \\
\hline 121 & 9 & -46 & 29 & 27 & 0 & 18 & 10 & -26 & 69 & 36 \\
\hline 022 & 9 & 73 & 58 & 20 & 2 & 18 & 10 & -46 & 5 & 54 \\
\hline 0 & 10 & 46 & 34 & 49 & 1 & 19 & 10 & -130 & 88 & 38 \\
\hline 0 & 10 & 56 & 5 & 49 & 0 & 20 & 10 & -103 & 41 & 39 \\
\hline 1 & 10 & -38 & 85 & 29 & 0 & 0 & 11 & -71 & 0 & 21 \\
\hline 1 & 10 & -38 & 45 & 32 & 2 & 0 & 11 & -40 & 0 & 47 \\
\hline 1 & 10 & -47 & 29 & 50 & 4 & 0 & 11 & -30 & 0 & 57 \\
\hline 2 & 10 & 36 & 39 & 27 & 1 & 1 & 11 & 100 & 67 & 18 \\
\hline 2 & 10 & -27 & 32 & 67 & 3 & 1 & 11 & -62 & 19 & 21 \\
\hline 2 & 10 & -51 & 72 & 49 & 5 & 1 & 11 & 94 & 76 & 19 \\
\hline 2 & 10 & -31 & 48 & 63 & 0 & 2 & 11 & 0 & 59 & 95 \\
\hline 3 & 10 & 55 & 48 & 24 & 2 & 2 & 11 & 52 & 44 & 25 \\
\hline 3 & 10 & 72 & 22 & 51 & 4 & 2 & 11 & -30 & 74 & 65 \\
\hline 3 & 10 & -28 & 28 & 37 & 1 & 3 & 11 & -69 & 68 & 48 \\
\hline 4 & 10 & 68 & 64 & 18 & 3 & 3 & 11 & 90 & 71 & 17 \\
\hline 4 & 10 & -27 & 59 & 65 & 5 & 3 & 11 & 68 & 42 & 50 \\
\hline 3 & 10 & 72 & 61 & 18 & 2 & 4 & 11 & 68 & 20 & 46 \\
\hline 5 & 10 & 0 & 52 & 90 & 4 & 4 & 11 & -26 & 65 & 37 \\
\hline 6 & 10 & -79 & 42 & 44 & 1 & 5 & 11 & 59 & 8 & 55 \\
\hline 6 & 10 & 50 & 69 & 29 & 3 & 5 & 11 & -96 & 56 & 38 \\
\hline 6 & 10 & -32 & 55 & 57 & 5 & 5 & 11 & -56 & 4 & 54 \\
\hline 7 & 10 & 43 & 9 & 61 & 2 & 6 & 11 & -98 & 3 & 35 \\
\hline 7 & 10 & -11 & 79 & 46 & 4 & 6 & 11 & -123 & 3 & 31 \\
\hline 8 & 10 & -97 & 53 & 36 & 1 & 7 & 11 & 30 & 29 & 65 \\
\hline 8 & 10 & 84 & 19 & 15 & 3 & 7 & 11 & 65 & 9 & 49 \\
\hline 8 & 10 & -30 & 58 & 62 & 0 & 8 & 11 & 51 & 32 & 62 \\
\hline 8 & 10 & 35 & 67 & 73 & 2 & 8 & 11 & 70 & 24 & 52 \\
\hline 9 & 10 & 65 & 47 & 24 & 4 & 8 & 11 & -78 & 65 & 51 \\
\hline 5 & 10 & -34 & 23 & 30 & 1 & 9 & 11 & -110 & 10 & 31 \\
\hline 010 & 10 & -95 & 42 & 40 & 3 & 9 & 11 & 12 & 39 & 46 \\
\hline 210 & 10 & 43 & 9 & 60 & 0 & 10 & 11 & -57 & 9 & 47 \\
\hline 10 & 10 & 33 & 14 & 70 & 2 & 10 & 11 & -68 & 35 & 40 \\
\hline 11 & 10 & 52 & 41 & 48 & 4 & 10 & 11 & 60 & 115 & \\
\hline
\end{tabular}


Reflexões não observadas $I<3 \sigma$ (I)

Values of $10 *$ Fobs and $10 *$ Fcalc

Page 25

\begin{tabular}{|c|c|c|c|c|c|c|c|c|c|c|c|}
\hline $\mathrm{H}$ & K & I & Fobs & Fcalc & $\operatorname{SigF}$ & $\mathrm{H}$ & K & $\mathrm{L}$ & Fobs & Fcalc & SigF \\
\hline - & - & - & ---- & ----- & ---- & - & - & - & ---- & ----- & ---- \\
\hline 1 & 11 & 11 & -90 & 15 & 38 & 2 & 4 & 13 & 59 & 82 & 59 \\
\hline 3 & 11 & $\therefore 2$ & -101 & 32 & 40 & 1 & 5 & 13 & 74 & 34 & 47 \\
\hline 0 & 12 & 11 & -78 & 11 & 40 & 0 & 6. & 13 & 60 & 62 & 25 \\
\hline 2 & 12 & 12 & 33 & 32 & 71 & 2 & 6 & 13 & -73 & 50 & 49 \\
\hline 3 & 13 & 11 & -92 & 20 & 33 & 1 & 7 & 13 & -92 & 12 & 17 \\
\hline 0 & 14 & $\because 1$ & 72 & 51 & 49 & 0 & 8 & 13 & 66 & 70 & 48 \\
\hline 2 & 14 & 12 & 76 & 76 & 45 & 2 & 8 & 13 & -99 & 8 & 43 \\
\hline 3 & 15 & $\because$ & -116 & 13 & 33 & 1 & 9 & 13 & -86 & 12 & 46 \\
\hline 1 & 17 & $\because$ & -103 & 95 & 44 & 0 & 10 & 13 & 75 & 4 & 48 \\
\hline 0 & 0 & $=2$ & 30 & 54 & 65 & 1 & 11 & 13 & 26 & 5 & 41 \\
\hline 2 & 0 & -2 & -68 & 40 & 45 & 0 & 12 & 13 & -73 & 16 & 42 \\
\hline 1 & 1 & -2 & 0 & 22 & 87 & 0 & 14 & 13 & 71 & 58 & 60 \\
\hline 0 & 2 & 12 & -64 & 50 & 45 & 0 & 0 & 14 & 47 & 36 & 61 \\
\hline 2 & 2 & -2 & 45 & 69 & 64 & 1 & 1 & 14 & 73 & 30 & 22 \\
\hline 1 & 3 & $=2$ & -41 & 83 & 63 & 0 & 2 & 14 & -85 & 16 & 19 \\
\hline 0 & 4 & 32 & 97 & 89 & 16 & 1 & 3 & 14 & 0 & 31 & 101 \\
\hline 2 & 4 & 12 & 61 & 59 & 23 & 0 & 4 & 14 & 76 & 36 & 45 \\
\hline 1 & 5 & 22 & 78 & 52 & 18 & 1 & 5 & 14 & -81 & 9 & 47 \\
\hline 0 & 6 & 12 & 69 & 5 & 45 & 0 & 6 & 14 & -50 & 13 & 27 \\
\hline 2 & 6 & 12 & -129 & 0 & 31 & 0 & 8 & 14 & -124 & 12 & 34 \\
\hline 4 & 6 & 22 & -88 & 36 & 36 & & & & & & \\
\hline 1 & 7 & $=2$ & 55 & 15 & 50 & & & & & & \\
\hline 3 & 7 & 22 & 34 & 14 & 67 & & & & & & \\
\hline 0 & 8 & $=2$ & 63 & 45 & 52 & & & & & & \\
\hline 2 & 8 & $=2$ & 69 & 30 & 22 & & & & & & \\
\hline 1 & 9 & 12 & -23 & 19 & 38 & & & & & & \\
\hline 3 & 9 & 12 & -87 & 41 & 44 & & & & & & \\
\hline 0 & 10 & \pm 2 & -60 & 5 & 52 & & & & & & \\
\hline 2 & 10 & 12 & -38 & 3 & 31 & & & & & & \\
\hline 1 & 11 & 32 & -31 & 49 & 35 & & & & & & \\
\hline 3 & 11 & 12 & 73 & 31 & 22 & & & & & & \\
\hline 0 & 12 & 12 & -93 & 59 & 39 & & & & & & \\
\hline 2 & 12 & 12 & -58 & 32 & 23 & & & & & & \\
\hline 1 & 13 & 12 & 87 & 66 & 20 & & & & & & \\
\hline 0 & 14 & 12 & -96 & 65 & 44 & & & & & & \\
\hline 1 & 15 & $\div 2$ & -57 & 37 & 55 & & & & & & \\
\hline 0 & 0 & 23 & -122 & 0 & 32 & & & & & & \\
\hline 2 & 0 & 23 & -45 & 0 & 59 & & & & & & \\
\hline 1 & 1 & 13 & 65 & 3 & 22 & & & & & & \\
\hline 3 & 1 & 13 & 78 & 118 & 23 & & & & & & \\
\hline 0 & 2 & 13 & -50 & 6 & 26 & & & & & & \\
\hline 2 & 2 & 13 & 75 & 125 & 53 & & & & & & \\
\hline$I$ & 3 & 13 & 35 & 23 & 33 & & & & & & \\
\hline c & 4 & 13 & -92 & 2 & 38 & & & & & & \\
\hline
\end{tabular}

